
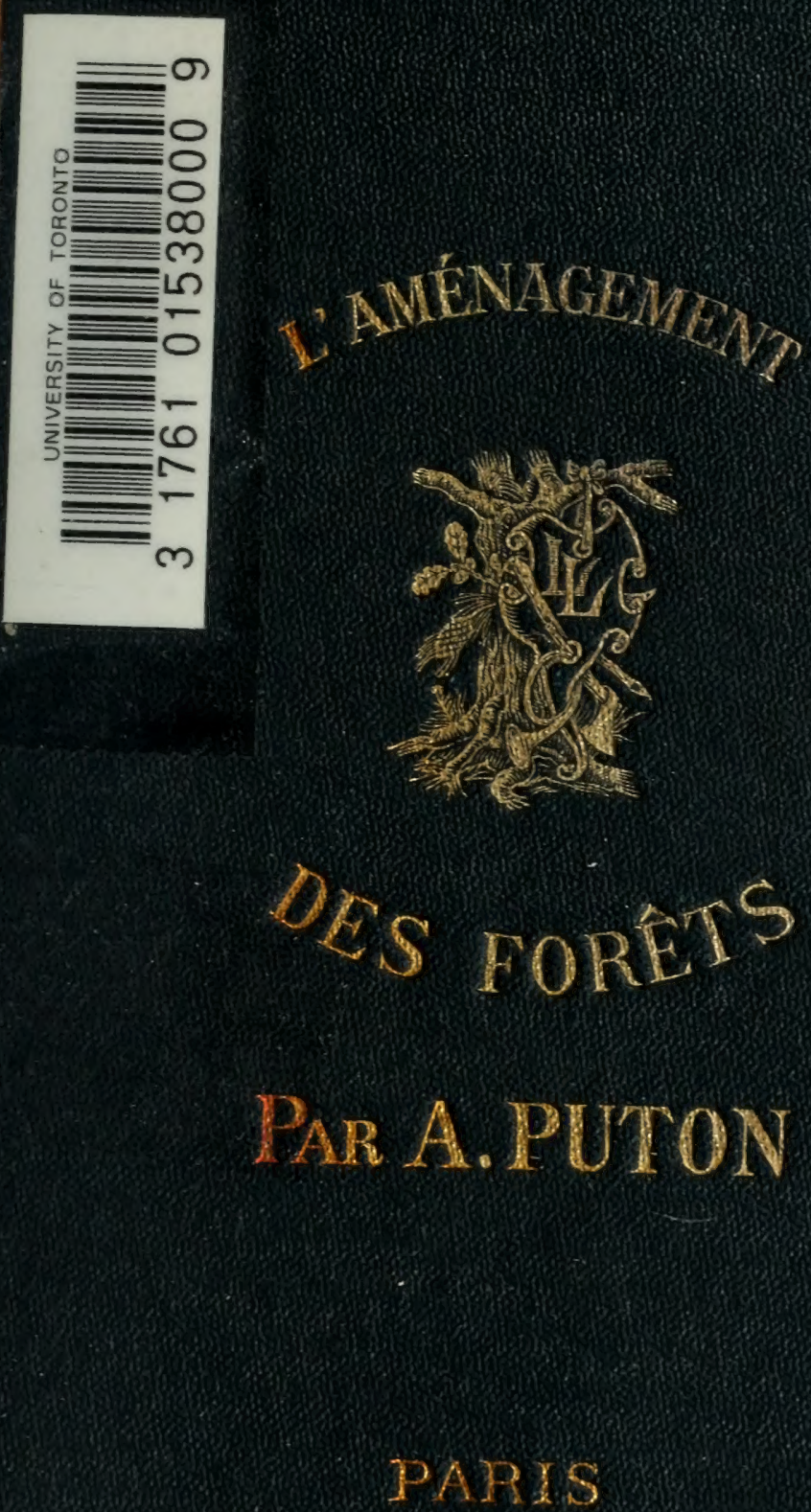



\section{L'A MÉNAGEMENT}

DES

F O R E T S 


\section{PARIS}

TYPOGRAPHIE GEORGES CHAMEROT

19, Rue des Saints-Pères. 19 


\section{L'AMÉNAGEMENT}

DES
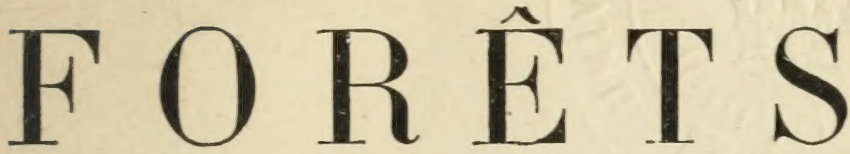

TRAITÉ PRATIQUE

DE LA CONDUITE DES EXPLOITATIONS DE FORETS

EN TAILLIS ET EN FUTAIE

PAR

ALFRED PUTON

Inspecteur général des Forêts

Directeur de l'École forestière de Nancy

TROISIÈME ÉDITION ORNÉE DE VIGNETTES
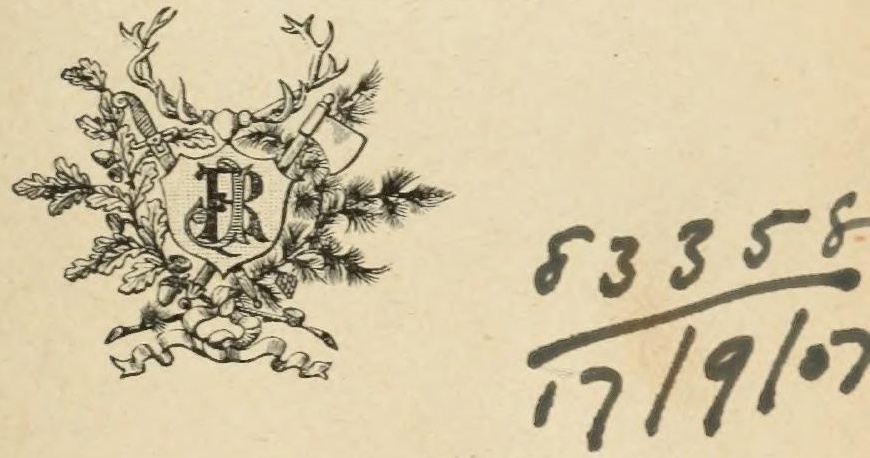

PARIS

J. ROTH C H I D, ÉDITEUR

13, RUE DES SAINTS-PÉRES, 13 
SD

431

P8

1884

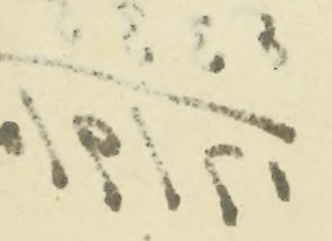


A LA MEMOIRE

$$
\text { D E }
$$

MII. LORENTZ ET PARADE 



\section{A}

\section{MONSIEUR A. LORENTZ}

Chevalier de la Légion d'Honneur.

Ancien Vérifieateur général des Aménagements,

Administrateur des Forèts.

\section{Movsieur,}

Les nombreux services que vous avez rendus, à tant de titres divers, à la science forestière, dont vous êtes le digne représentant, et l'éminente fonction que vous avez occupée dans l'aménagement des forêts francaises, devaient naturellement me porter à vous dédier ce petit écrit dans lequel j'ai essayé de suivre vos leçons.

Puisse cet hommage vous redire avec quel bonheur je conserve le souvenir de vos visites annuelles dans nos forêts, et des conseils affectueux que vcus donnez à tous les forestiers.

Puisse-t-il aussi vous offrir l'assurance nouvelle des sentiments de respect

avec lesquels

j'ai l'honneur d'ètre

Votre très dévoué Serviteur,

A. Puton. 
Digitized by the Internet Archive in 2010 with funding from University of Ottawa 


\title{
AVERTISSEMENT
}

\author{
DE LA PREMIER ĹDITION.
}

Les principes de la culture forestière sont maintenant, dans les pays forestiers, connus de tout le monde: propriétaires et régisseurs, gardes et bûcherons savent tous ce que c'est qu'une coupe d'ensemencement, secondaire, définitive, d'éclaircie, etc. Depuis quelques années, une grande impulsion a été donnée aux travaux d'aménagement. Des Commissions d'agents forestiers ont parcouru les forêts, traçant des lignes, étudiant les peuplements, créant des parcelles, des affectations, parlant de périodes, de coupes d'abri, de conversions, établissant des règles de gestion auxquelles les gardes ne comprenaient rien. J'avais été attaché, pendant trois années, à l'une de ces Commissions, dirigée par des Inspec- 
leurs de grande expérience, et les gardes me demandèrent un jour de leur expliquer ce que c'est qu'un - Aménagement. J'y consentis, mais je m'aperçus bientòt que la tîche était assez difficile, et qu'il n'existait pas de livre à leur portée. C'est pour eux spécialement, c'est pour toutes les personnes qui administrent des forêts, que ce petit livre est publié. II n’apprendra rien de neuf aux forestiers ni aux gens du métier; mais s'il peut contribuer à vulgariser les saines notions de l'économie forestière et les principes qui servent à diriger une exploitation raisonnée de forêts, mon ambition sera satisfaite.

A. Puton. 


\title{
A VERTISSEMENT
}

\author{
DE LA SECONDE LEDTION.
}

Le bienveillani accueil fait à ce petit livre a engagé l'éditeur à en publier une seconde édition. Je n'ai jamais eu la prétention d'écrire un traité d’aménagement, pour lequel il faut un savoir auire que le mien. ct je désire préciser, plus encore que je le faisais en $1 \$ 66$. le caractère et le but de ce petit écrit.

Pour régir et administrer convenablement une forèt, il faut connaitre la culture de ses essences, saroir laménager et comprendre le méranisme de sun aménagement. La sylviculture et l’aménagement sont deux branches de la science forestiere intimementliées, s’éclairant et se fortifiantl'une par l'autre, mais correspondant à des idées distinctes.

Ju suppose acquises toutes les connaissances culturales: - elles ont leurs livres et leurs auteurs spéciaux. 
Je n'ai pas pour but d'enseigner ni mème d'indiquer les procédés qui servent à faire l'aménagement d'une forêt; - c'est la matière de tout un traité el

- d'un Cours qui a été publié par M. Nanquette, le savant directeur de l'École forestière de Nancy.

J'aborde encore moins l'étude des théories el la discussion des doctrines; - il y a, sur ce point, des livres parmi lesquels on doit citer les $\dot{E} t u d e s$ sur l’Aménagement des Forêts, de M. Tassy.

Mon but, essentiellement restreint, est d'indiquer le mécanisme des méthodes les plus usuelles d'aménagement, d'expliquer, en un mot, ce que c'est qu'un Aménagement et comment on établit les comptes d'une gestion de forêt en les fondant sur son aménagement.

Ce livre d'Administration pratique tend à répandre dans la gestion des forêts les idées d'ordre et de comptabilité dont les fortunes particulières ne sauraient pas plus se passer que la fortune publique. C'est à ce côté pratique, bien plus qu'à des notions scientifiques trop souvent incomplètes, que doivent, à mon sens, être limités les livres élémentaires.

A. Puton. 


\section{L'A IÉNAGEMENT DES F0RÊTS.}

\section{PREMIĖRE PARTIE.}

Wotions d'économie forestière.

\section{CHAPITRE PREMIER.}

DE L'EXPLOITATION DES FORÊTS EN GÉNÉRAL.

Capital d'exploitation. - Pour exploiter un terrain cn forêt, comme pour l'exploiter en champ ou en pré, il faut, de toute nécessité, un capital, que les économistes ont appelé capital ou matériel d'exploitation. En agriculture, ce capital est formé par les bâtiments de la ferme, par les semences, par le bétail et les instruments de culture; une partie est immeuble, l'autre partie est généralement meuble. En sylviculture, au contraire, le capital d'exploitation est toujours immeulble; il est formé par un certain volume de bois représentant une échelle d'àges graduée de 1 à 20 ans, par excmple, pour un taillis qu'on exploite à 20 ans, ou die 1 ì 120 ans pour une futaie, si on exploite celle-ci à ce dernier âge. 
On conçoit dès lors la différence qui existe entre la propriété agricole et la propriété forestière: en agriculture, si l'exploitant fait mal ses affaires, ou si des circonstances l'obligent à liquider, il ne peut généralement vendre les bàtiments sans la terre elle-même; car, sans elle, ils n'auraient point de raleur. En sylviculture, au contraire, l'exploitant peut faire argent de tout le matériel-bois qui se trouve sur la propriété, et alors la terre reste sans livrer de produits pendant longtemps, pendant tout le temps qui est nécessaire à la nature pour reconstituer des bois exploitables. Ceci explique comment il est nécessaire qu'une administration publique veille à la conservation du matériel d'exploitation dans les forêts de l'État et dans celles des communes et des établissements publics; car ces êtres moraux ne sont, dans l'ordre social, que des usufruitiers et doirent transmettre aux générations futures la propriété munie de son matériel d'exploitation complet. L'intérêt public aurait trop à souffrir, si pour une circonstance quelconque, même très-urgente, on renait à l'entamer d'une manière générale, car un pareil capital ne se reforme pas avec de l'argent comme les batiments d'une ferme, mais par le temps seul et par un temps souvent fort long.

Notons encore une autre différence entre le capital d'une exploitation agricole et le matériel dectiné à 
l'exploitation d'une forêt: en agriculture, le propriétaire qui a besoin d'argent peut vendre son bétail, ses charrues, ses voitures; généralement, ces instruments d'exploitation sont achetés par des cultivateurs et vont servir à monter une autre exploitation agricole. Un individu peut être gêné, mais la société n'en souffre pas. Le propriétaire d'une forèt, au contraire, est toujours tenté, quand de mauvais jours surviennent, de vendre une partie des boisqui forment l'échelle d'âges nécessaire pour une bonne exploitation ', et il arrive un double malheur. Le premier est que le matériel vendu ne va pas à une autre exploitation; il y a diminution dans le nombre des outils qui travaillent dans un pays à la production ligneuse. Le second vient de ce que, la portion de bois prise sur le matériel d'exploitation élant vendue en chauffage ou en bois de service, l'offre de la marchandise peut dépasser la demande. La production peut n'être. plus en rapport avec la consommation; et, les prix s'arilissant, l'intérêt public souffre, parce que les propriétaires voisins n'obtiennent plus un prix rémunérateur, parce que les usines qui usent du bois

1 Le mot exploitation a une autre acception plus restreinte et désigne l'opération qqui consiste à couper, à façonner et à vider les bois d'une coupe. Nous l'emploierons rurement dans ce dernier sens; il aura dans la suite, comme ici, le sens économique de : faire valoir, conduire une entreprise agricole, forestière ou industrielle. 
sont un instant dans l'abondance pour le payer plus tard trop cher, quand la rareté sera venue, parce qu'enfin une denrée précieuse, en ce sens que le temps seul, et non la main des hommes, en est l'ourrier, a été avilie et gaspillée.

Il n'est nul hesoin d'être agronome pour saroir qu'il y a dans tous les pays, la grande et la petite culture, l'une traraillant sur quelques champs arec de simples outils et quelques engrais achetés, l'autre constituée par des hâtiments d'exploitation, des machines agricoles, une certaine étendue de terres. Il y a aussi la grande et la petiłe cullure de forêts: il $\mathrm{y}$ a les futaies, qui se reproduisent par les semences, et les taILlis, qui se reproduisent pur les rpjets des souches. Cette division correspond aux exploitations de forêts constituées par un gros ou par un petit capital d'exploitation, parce que les arbres n'arrivent, en général, à produire des semences qu'à un âge arancé, entrainant ainsi une longue échelle d'ìges, un matériel considérable, tandis que la faculté qu'ont certaines essences de produire des rejets permet d'exploiter une forèt dans un court espace de temps et arec un faible matériel sur pied. Ce fait peut se constater pour deux terres de 120 hectares traitées, par exemple, l'une en taillis a 20 ans, l'autre en futaie à 120 ans.

Pour la futaie. Supposons que l'hectare de bois produise 
600 mètres cubes 1 ì l'âge de 120 ans; le matériel d'exploitation des 120 hectares sera formé par une suite de peuplements âgés de 1 ì 120 ans, dont le volume suit une échelle ascendante de zéro à 600 mètres cubes, soit une moyenne approximative de 300 mètres cubes. On peut donc représenter le volume du matériel d'exploitation par

$$
120^{\mathrm{h}} \times 300^{\mathrm{mcc}}=36,000^{\mathrm{mcc}} \text {. }
$$

Pour le taillis. Un hectare de taillis de 20 ans pouvant fournir 50 mètres cubes, le matériel d'exploitation, e'està-dire le rolume des bois âgés de 1 ì 20 ans, s'éraluera par une semblable moyenne approximativement à

$$
120^{\mathrm{h}} \times 25^{\mathrm{mcc}}=3000^{\mathrm{mcc}} .
$$

i.e producteur de bois par le moyen de la futaie derra posséder un capital de 36,000 mètres cubes, tundis que son roisin, producteur de bois par le régrime du taillis, pourra exploiter la mème terre avec un ontillage douze fois moindre.

On roit done déji qu'il faut ètre très-riche pour produire du bois par le moyen de la futaie et que l'État el les communes sont, en France, d'une manière générale du moins, les seuls propriétaires assez riches pour tenir une aussi grosse culture, assez à l'abri du besoin pour n'ètre pas tentés à chaque instant de mettre la main sur le matériel d'exploitation, et virant assez longtemps pour pouvoir attendre la formation d'un aussi gros capi-

I L'unité adoptée est le mètre cube, volume réel, plein, ou mètre cube en grume des forestiers. Le mot stere ne s'applique qu'aux bois de chau fiage il ne représente qu'une quantité incléterminée, variant avec l'empilage, ef n'est susceptible d'aucune comparaison. 
til. Si l'on veut classer, à cet égard, les exploitations des terres à bois, on peut dire que la propriété privée forme la petite culture et que les ètres moraux impérissables sont seuls susceptibles de la grande culture forestière.

Il y a pour ce classement d'autres raisons non moins puissantes.

Les produits de l'agriculture sont toujours les mèmes, qu'ils vierment du champ de l'artisan ou du domaine du grand propriẻtaire : ce sont toujours des blés, des fourrages, du bétail. Dans les forèts, les choses se passent autrement: les taillis ne produisent guère que de la charbonnette, des fagots et des menus bois; aux futaies seules est réservée la production des planches, des traverses de chemin de fer et des bois de service. Or l'une et l'autre production sont également nécessaires au pays, et comme il n'y aurait peut-être pas de propriétaires assez riches pour avoir, et surtout pour garder, des futaies produisant des bois. de fortes dimensions, l'intérèt public fait un devoir à l'État el aux communes de posséder des exploitations de forèts capables de fournir à la société ces objets de première nécessité.

Production. - La production générale et, par suite, la richesse d'un pays gragnent et s'accroissent par la grande culture forestière. Si, en effet, nous 
revenons aux deux exemples déjả cilés et qui seront conservés dans la suite pour la facilité du raisonnement ', on voit que le taillis peut rapporter, par hectare et par an, $21 / 2$ mètres cubes qui, à raison de 8 francs l'un, portent la production annuelle à 20 francs par hectare.

$$
\frac{50 \mathrm{mcc}}{20 \mathrm{ans}}=2^{\mathrm{mcc}, 500}
$$

Le même bois traité en futaie peut rapporter au contraire cinq mètres cubes par hectare,

$$
\frac{600^{\mathrm{mcc}}}{120 \text { ans }}=5^{\mathrm{mcc}}
$$

et si l'on y ajoute le produit des éclaircies (éralué pour le moins à 15 p. 100 en sus), on arrive à $5^{\text {mcc }}, 750$ de production annuelle, dont la valeur s'élève sourent, à raison du bois de service, à $15 \mathrm{fr}$. par mètre cube, de sorte que la terre rapporterait par an 81 francs par hectare:

$$
\begin{array}{r}
5_{\text {mec }} \times 15^{\mathrm{f}}=75 \mathrm{fr} \\
0,750 \times 8=\frac{6}{81} \\
\text { Total. } \ldots .
\end{array}
$$

On a calculé que si toutes les forêts qui existent en France étaient traitées en futaie, on arriverait à

1 Les taillis s'exploitent à des âges qui rarient de 6 à 30 et 35 ans, selon les essences. Il faut 150 et 200 ans pour produire un chêne ou un sapin de bonne dimension, 300 ans pour certains mélezes des Alpes. On peut dire que les révolutions de 120 ans sont, en général, trop faibles pour nos principales essences. 
augmenter, dans l'avenir, leur production de 15 . millions de mètres cubes de bois et de 290 millions de francs. C'est plus de la moitié de nos contributions directes, c'est plus que l'intérêt de notre con. tribution de guerre ${ }^{1}$.

Mais sur huit millions d'hectares de forèts qui existent en France, l'État en possède à peine un million, les communes deux; el les forèts des particuliers sont, pour cing millions d'hectares (près des trois quarts de la contenance totale) exploitées presque uniquement en taillis.

Rente. - Comment se fait-il donc, puisque les futaies rapportent plus en matière et en aryent que les taillis, que les particuliers possèdent si peu de futaies et s'en tiennent généralement aux taillis?

La raison en est fort simple:

Le particulier traraille pour son compte; il n'est pas plus tenu de fournir du bois au pays, gu'il n'est obligé de procurer du travail aux ouvriers, ou des matieres premieres à l'industrie. Il ne produil du bois que parce que le bois lui produit de l'argent ef parce qu'il trouve dans la forèt un placement avautageux et sûr. Eh bien! comptons, et reprenous notre exemple: en évaluant à 300 francs par hectare le sol de nos 120 hectares de forèt, nous aurons,

1 M. Tassy, Études sur l'aménagement des forêts, 2c édit., p. 169. 
pour l'éraluation approximative du capital eng̨agé dans l'exploitation en taillis :

10 Sol, 120 hectares, à $300 \mathrm{fr}$. . . . . $36,00 \mathrm{fr}$.

20 Matériel-bois, 3000 mètres cubes, à $5 \mathrm{fr}$., prix calculé ì raison des taillis en croissance.

Total

$\frac{15,000}{51,000 \mathrm{fr} .}$

Les prodnits sont: $\bar{b}$ hectares par an, it 50 mitres cubcs lun, soit 8 min mètres cubes, éralués $8 \mathrm{fr}$. . . $240 \mathrm{fr}$.

De surte que le propriétaire a son argent placé à envi. ron $41 / 2$ p. 100 .

Far-ons le mème calcul pour la futaie; le capital engage se compose:

- $1^{\circ} \mathrm{Du}$ sol .

$36,000 \mathrm{fr}$.

$2^{\circ}$ De la valeur en areent des $2 \pi, 000$ mitres cubes formant le matériel-bois, suit ì $11 \mathrm{fr}$. l'un

Total

$\frac{396,000}{432,000 \mathrm{ir}^{\circ}}$

Les produits sont:

10 Lin hectare de eon mitres culses, it $15 \mathrm{fr}$. $9000 \mathrm{fr}$.

$2015 \mathrm{l} .100 \mathrm{c}$ cn sus putr les éclaircies, suit 90 mètres cubes, à 8 fr.

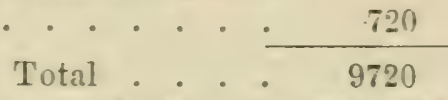

De sorte que les 432,000 fi: rapportent environ 2 p. 100.

On voit done que la futaie, tout en pourant produire prés de trois fois plus en volume et cing fuis plus en argent que le taillis, fournit au propriétaire une rente bien plus liaible, quand on compare la production au capital engagé. Or, dans notre societe, lien peu de personnes sont assez riches pour se contenter de fuire rapporter 20,0 , et sourent moins, à leur argent. 
$\mathrm{Si}$, par un concours de circonstances, une famille est assez riche pour se contenter de ce faible taux de placement, il n'y a nulle témérité à avancer que cet état ne durera pas au delà de deux ou trois générations. Sans parler des revers et de l'instabilité qui caractérisent les fortunes particulières, il arrivera que des partages de descendants viendront démembrer une exploitation dont le temps et la fixité sont les conditions essentielles.

Il arrivera d'ailleurs que les particuliers feront le calcul suivant:

Le possesseur d'une futaie de bois feuillus de 120 hectares ayant un matériel de 36,000 mètres cubes, peut réaliser tout ce qui n'est pas nécessaire pour constituer une forêt de taillis, c'est-à-dire

$$
36,000^{\mathrm{mcc}}-3000^{\mathrm{mcc}}=33,000^{\mathrm{mco}} ;
$$

cette réalisation lui procurera, à 10 fr. environ par mètre cube, une somme de $330,000 \mathrm{fr}$. S'il veut continuer à avoir sa fortune placée en forêt, il pourra, arec cet argent, acheter plus de six fois autant de taillis, dont la valeur capitale n'est que de 51,000 fr. par 120 hectares. Il aura ainsi:

10 Son ancienne forêt, $120^{\mathrm{h}}$, valant $51,000^{\mathrm{f}}$, rapport. $2,400^{\mathrm{f}}$

$2^{\circ}$ Son acquisition. $\frac{775}{895} \quad \frac{330,000}{381,000} \quad \frac{15,500}{17,900}$

Ainsi, à la place d'une forêt de 120 hect. valant $432,000 \mathrm{f}$. et rapportant $9720 \mathrm{fr}$., il aura 895 hect. valant $381,000 \mathrm{fr}$. et rapportant $17,900 \mathrm{fr}$ : : sept fois plus de terre et près du double de revenus! et cela avec la même sécurité et la même nature de placement.

Quel est le propriétaire qui hésiterait? 
Mais ces 895 hectares traités en taillis ne livreront à la société que 2235 mètres cubes de matière à consommer et à travailler (2mcc, 500 par hectare) tandis qu'exploitées en futaie, ces mèmes terres pourraient produire 5145 mètres cubes (5 ${ }^{\text {mcc }}, 750$ par hectare) de matière lien autrement précieuse par sa valeur et par la quantité et l'utilité du travail qui en résulterait. La futaie aurait lirré à la société trois fois plus d'éléments de travail et d'industrie, cinq fois plus de valeurs d'utilité et d'échange. Or en quoi ces considèrations importent-elles aux particuliers uniquement préoccupés du placement de leur argent?

Aussi toutes les futaies possédées jadis par la nollesse et par l'Église, qui ont èté confisquées et vendues à la Rérolution, toutes les forêts de l'État que des alienations successives ont fait entrer dans le commerce, ont-elles été ramenées, sauf de rares exeptions, à des exploitations à petit capital, seules compatibles arec l'état de la fortune des particuliers.

Remarquons, en outre, que le capital d'exploitation destiné à la production des bois de service une fois détruit, ne se reconstitue pas comme celui d'une exploitation agricole ou comme l'outillage d'une usine. Arec de l'argent, on remonle ceux-ci en une seule annéc; à l'industrie forestière, il faut un temps très-long, un temps presque toujours séculaire pour reproduire son capital. 
Il est donc vrai de dire qu'en industrie forestière l'intérèt des particuliers est contraire à la conservation d'une exploitation en futaie et qu'ils sont incapables de devenir producteurs soutenus de bois de fortes dimensions.

Ròle des forêts de l'État. - Cette situation économique de la propriété particulière est aujourd'hui consacrée par les modifications introduites successivernent diuns son régime légal. L'ancien ordre successoral qui tendait à conserver dans certaines familles les grands patrimoines est aboli et la législation moderne soumet tous les héritages à la loi d'un morcellement indéfini.

Notre ancien droit coutumier, en immobilisant les arbres de futaic et en les attachant au sol dont ils derenaient en quelque sorte partie intégrante, les faisait échapper a l'action destructive des usufruitiers, des usagers et de tous ceux qui ont sur les immeubles une jouissance temporaire. La législation, devenue positive comme la société elle-même, n'impose plus aux jouissances temporaires d'autre règle que celle de la liberté des conventions.

Le droit de préemption, donné ả la marine sur les bois des particuliers et aboli en 1837, faisait que les ressources des forêts domaniales se ménageaient au profit des générations futures.

Tout cela a disparu et devait disparaitre dans une 
société démocratiquie qui a pour caractère une immense expansion vers le travail et l'échange. Dans notre France, comme dans toutes les sociétés modernes, il n'y a donc que les ètres moraux impéris sables qui sont assez riches et surtout assez viraces pour consacrer leurs forêts à la production des bois de fortes dimensions dont le travail national ne pourra jamais se passer, malgré l'emploi du fer dans les constructions.

Or, de ce côté, il y a aussi bien des changements.

Sous l'ancienne monarchie, il y arait trois natures d'êtres moraux impérissables ayant le droit illimité de propriété: l'État, les communes et l'Église. Cette dernière ayant perdu, depuis la Révolution, la liberté de posséder, au moins pour les communautés religricuses, l'État reste seul, avec les communes, en qualité de grand propriétaire. Nos puissantes compagnies financières sont, en effet, bien plus des sociètés de irédit et de travail que des associations d'épargne; clles sont loin d'ailleurs d'ètre impérissables.

Les communes propriétaires de forêts étaient jiulis soumises par une antique et nationale législation a l'obligation de consacrer un quart de leurs propriétés à élever des futaies dans l'intérêt général. Rendues à la liberté et ramenées à n'ètre plus 'qu'une simple manifestation de l'intérèt local, les communes françaises ont été dispensées de l'obli- 
gation de fournir cette prodiıction coûteuse. Leur quart de réserve n'est plus qu'une simple mesure de préroyance que le Code de 1827 leur impose et dont sourent même il leur permet de se dispenser. Allez donc demander aujourd'hui à une commune des Pyrénćes de laisser croître un quart de ses bois en futaie et de s'interdire d'y toucher pendant un siècle sous prétexte que les populations du nord ou de l'ouest peurent aroir besoin d'arbres séculaires ! Cela n'est plus dans des mœurs qui ont songé, un instant, a ériger l'égoïsme local à la hauteur d'un principe social.

L'État reste donc seul capable en France de consacrer ses forèts à la production des grands bois, matière indispensable dont le temps et l'épargne sont les seuls ouvriers.

Ajoutons que c'est pour lui une obligation impérieuse, un devoir national.

Supposez un instant que par suite d'une vente générale, si sourent conseillée et si souvent repoussée par le bon sens public, toutes les forèts de l'État aient passé aux mains des particuliers. Pour que ceux-ci aient intérèt à conserver à la consommation des richesses accumulées qui constituent une si conteuse exploitation, il faudrait que le prix des produits rint à s'élever d'une facon calamiteuse; on a calculé, en effet, que, pour donner au propriétaire 
un taux conrenable, le prix de revient du mètre cube de bois de service serait de plus de 300 francs, et encore ce taux ne serait jamais bien élevé, car le capital d'exploitation augmente de valeur à mesure que la valeur du mètre cube augmente.

Une pareille situation ne pourrait subsister, et les bois venus de l'étranger, faisant sur nos marchés concurrence aux bois indigènes, les particuliers cesseraient bien vite une exploitation si peu remunératrice, si inconciliable avec leur intérêt. Les forèts de France, ramenées à la petite culture forestière, ne livreraient plus à la consommation, comme le proure l'exemple du passé, que des bois de menu chauffage et de petit service.

Il faudrait avoir recours aux violences de la loi; il faudrait limiter les prix par un maximum, et forcer les particuliers, par des peines sévères, à conserver leurs futaies tout en continuant à recevoir les bois de l'étranger, situation pleine d'injustice qui ferait peser sur les uns un impòt inique, car on les forcerait à vendre aux autres, à très-bon marché, ce qui leur coûte très-cher à produire.

Ou bien il faudrait interdire le marché français aux bois étrangers, entourer la patrie d'une de ces murailles prohibitives que réprourent les lois économiques du travail et qui arrètent le développement des sociétés modernes. 
Que l'on necroie pas à l'existence de forêts vierges, inexploitées dans des pays étrangers, oủ le commerce ira alimenter le travail national. Certains lives ne manquent jamais de parler de ces mines inépuisables à l'appui d'étranges et captieuses théories. Aucun ne cite l'emplacement de ces greniers d'abondance, et dans la vieille Europe, comme dans le Noureau-Monde, le cri d'alarme commence à retentir'. Eh bien, supposez qu'il existe en quelque heureux pays des ressources forestières assez puissantes pour subvenir aux besoins incessants de nos chemins de fer, de notre marine, de nos rignes, de nos constructions, de tous les besoins de notre dévorante civilisation. Supposez également qu’à la suite de la vente de toutes les forèts de l'État, les forêts soient réduites à ne fournir que des taillis et des bois de feu. Est-ce que, dans ces conditions, les Etats assez heureux et assez sages pour conserver leurs chênes et leurs sapins ne pourront pas nous les faire payer fort cher? Ne pourront-ils pas, en frappant leurs Jois de droits de sortie, lever sur la France le plus désastreux des impôts? lui faire la guerre la plus terrible, la guerre au travail?

Voilà donc où nous conduirait la vente générale des forêts de l'État:

1 Voir un article publié dans la Retue des Deux-Monules, en 1871, par M. Broilliard professeur à l'École forestière, sur la réserre des chênes d'arcniz. 
Arec la liberié du commerce à l'exportation et à l'importation : la destruction de nos richesses forestières, la mise du travail français aux mains de l'étranger!

Arec la liberté de l'importation, mais la contrainte des propriétaires de forèts : un impòt inégal, inique, pesant sur les uns au profit des autres.

Arec la prohibition d'entrée des bois étrangers: une elération effrayante dans le prix des bois d'ourre, calamiteuse pour notre travail, un impòt noureau sur le consommateur, impòt d'autant plus dur ì supporter qu'il est impossible d'en prévoir les conséquences $\boldsymbol{t} \mathbf{t}$ d'en mesurer les effets.

C'est une production coûteuse que celle des grands bois; elle coute beaucoup de temps et beaucoup d'argent; l'Etat peut seul l'entreprendre, et il doit la faire sur toutes les forèts qu'il possède.

Il est temps de songer que les forèts de l'État sont destinces à produire du bois, des grands bois surtout, et non de l'argent. La Rérolution a trouvé l'État riche de deux millions et demi d'hectares de forèts; après 84 ans, le domaine est réduit à 900,000 hectares. Il est temps de penser à l'arenir! 


\section{CHAPITRE II.}

\section{DE L'AMÉYAGEMENT EY GÉYÉRAL.}

Définition. - Je me suis peut-être trop étendu sur ces considérations; mais mon but a été do montrer combien est utile, combien est commandée par l'intérèt général la conservation du capital d'ex ploitation qui assure la production forestière. Là es le véritable ròle de l'administration forestière, qui ne doit livrer à la consommation que la production e ne jamais entamer le capital. L'aménagement es une opération qui a pour résultat de permettre l'exécution facile de ce but élevé.

On en concoit tout de suite la nécessité, car dans une masse de forèts, il n'est pas facile, au premiè abord, de distinguer ce qui est production de ce qui est capital, puisque, l'un et l'autre étant du bois, la coupe anmuelle n'est, en réalité, qu'une partic du capital rendue libre par la végétation de chaque année.

L'aménagement est une opération qui a poui but de déterminer le matériel d'exploitation qui convient le mieux aux intérêts du promiétaire, et de régler la nature, la quotité, l’ordre et la marche des coupes qui, tout en laissant intact ce matériel, constituent la production anmuelle. 
Celte définition a besoin de quelques explications pour ètre bien comprise. Avant de les donner, il est nécessaire d'entrer dans certains détails.

Anciennes méthodes. - L'aménagement est une opération aussi distincte de la culture forestière qu'en industrie la conduite de l'affaire est distincte de la fabrication, et qu'en agriculture l'assolement et la constitution de la ferme sont distincts du travail agricole. La grande différence, c'est qu'en industrie on ne peut jamais confondre les produits avec le rnatériel d'exploitation; en agriculture, la confusion est quelquefois possible; en sylviculture, elle est toujours à craindre.

La question que doit se poser le propriétaire d'une cxploitation forestière se réduit à un problème d'un énoncẻ très-simple: Une forêt doit-elle s'exploiter à cent vingt ans? La partie à couper tous les ans est la production annuelle, c'est-à-dire l'accroissement du matériel-bois qui est constitué par une échelle d'îges graduée de un à cent vingt ans. Si done on pouvait déterminer, d'une manière exacte et précise, l'accroissement annuel, la question serait résolue; car, en coupant tous les ans le volume ainsi exactement déterminé, on serait certain de laisser toujours le matériel intact. Mais on ne connait pas encore la loi de la végétation forestière, et les travaux qui ont élé faits sur cette grave question n'ont point 
encore acquis ce degré de certitude qui leur donn une portée pratique.

Les anciennes méthodes d'aménagement de Hartig, des de Salomon étaient fondées sur cett solution directe: on déterminait, par des calcul d'accroissement, le volume que pouvait fournir jusqu'au moment de l'exploitation, chacune des dif férentes parties de la forêt; on faisait masse du vo lume tolal à recueillir pendant les cent vingt ans d la révolution, et la cent-vingtième partie de ce volum formait la production annuelle que l'on devait prendr sur les points où se manifestaient les exigences cul turales.

Ces méthodes plus ou moins compliquées, basée sur des lois d'accroissement plus ou moins hypothe tiques, n'ayant point donné les résultats qu'or pouvait désirer, on a renversé la question; et, ne pouvant déterminer d'une manière précise l'accrois sement annuel du capital d'exploitation, c'est-à dire le volume à couper, pour le laisser toujour intact, un a cherché à déterminer le capital lui. mème, ou plutòt un cadre, une combinaison telle que l'on soit sûr que les exploitations ne l'entame. ront jamais.

On verra plus loin que cette combinaison se bor. nera, pour les taillis, à une simple division de contenance en autant de coupes que d'années, e 
pour les futaies, à de larges coupures faites sur le terrain pour correspondre à un certain nombre d'années avec l'estimation du volume des bois actuellement exploitables: c'est à MM. Lorentz et Parade qu'est due, en France, cette méthode simplifice si ficile ì appliquer, si fẻconde en résultats; c'est à MII. Tassy et Nanquette que l'ôn doit d'en aroir étendu les applications et développé la pratique.

Matériel d'exploitation. - Avant d'entrer dans l'examen des combinaisons qui constituent les méthodes d'aménagement, je dois insister sur la manière dont est constitué le capital d'exploitation des forèts ${ }^{4}$.

Un propriétaire de 120 hectares de terrain veut-il produire d'une manière soutenue, annuelle, des bois âgés de 120 ans? il devra posséder :

1 hectare peuplé de bois de 1 an

1 hectare id. de 2 ans

1 hectare id. de 3 ans

et ainsi de suite, de sorte qu'en exploitant le centvingtième hectare âgé aujourd'hui de cent vingt ans, il pourra exploiter l'année suivante le cent-dix-neuvième qui a actuellement cent dix-neuf ans, mais aura, l'an prochain, cent vingt ans, etc., etc. Si ce

1 Nous ferons remarquer que les mots ccapital et matéricl d'exploitation" seront toujours employés concurremment et cornme synonymes, sans que le premier entraîne aucune idée d'argent. 
propriétaire possède ainsi cent vingt hectares dont les peuplements soient en gradation d'àge et dans l'état de végétation correspondant à chaque îge, il aura une forèt capable de lui fournir tous les ans un revenu parfaitement soutenu, une exploitation dont le matériel sera parfaitement constitué.

Trois éléments entrent donc dans la constitution du capital d'exploitation: l'état de végétation, la contenance et l'äge. Il faut que les àges soient gradués de un à cent ringt ans; que les bois de chaque àge occupent des contenances égales ou proportionnées aux différences qui existent dans les conditions de la régétation dans la propriété; qu'il y ait enfin, pour chaque âge, un état de peuplement ayant la consistance et le volume conformes aux lois de la végétation dans la circonstance donnée. Car il faut que, tous les ans, la coupe porte sur une contenance équiralente au cent-vingtième de la forêt — sur l'àge de cent ringt ans - et sur cette partie du rolume que l'on ne peut déterminer $\dot{a}$ priori et qui est l'accroissement.

Il est nécessaire d'entrer dans quelques détails sur chacun de ces trois éléments pour bien faire comprendre les conditions économiques de l'exploitation forestière. Nous simplifierons ces détails en supposant que les conditions générales de la régétation sont les mèmes dans la localité où est située la forèt. 
DE L'AMENAGEMENT EN GENERAL. 23

Nous nous affranchirons ainsi de l'obligation de proportionner ces trois éléments entre eux ${ }^{1}$.

Relativement à la contenance, il est facile de comprendre que pour une terre dont le sol, le climat, la situation sont sensiblement les mêmes, des contenances égales produiront égale quantité de bois. Celui qui couperait, tous les ans, un des hectares de l'exemple qui nous occupe, en commençant par le dernier et en le repeuplant immédiatement après la coupe, aurait le capital d'exploitation le mieux et le plus simplement constitué. Il en résulte qu'il faut une certaine étendue de terres pour former une exploitation complète de forèt. Il faul, en effet, pouvoir cultiver convenablement les bois de chaque catégorie et les traiter suivant les exigences qu'ils ont à chaque âge de leur vie. Le véritable producteur de bois doil posséder une terre assez grande pour que chaque classe d'âge ait un emplacement suffisant. Celui qui ne possède que quelques arbres n'a pas une exploitation constituée dans le sens de ce que nous appelons une forêt. Pour les forêts de faible étendue, on a imaginé un mode spécial, le jardinage; mais, mème dans ce cas, les règles d'exploitation reposent

1 On a imaginé un certain nombre de combinaisous d'aménagement pour proportionner les éléments du capital d'exploitation, la contenance à la fertilité, le temps au volume. Ces méthodes sont peu usitées, et l'établissement des síries d'exploitation, dont nous parlerons plus loin, fournit un moyen tries-simple et, en général, suffisant pour parer a de trop grandes négralités dans les conditions de la végétation. 
toujour's sur le principe que le matériel d'exploitation n'est constitué que lorsque les bois de chaque àge occupent leur part respective de la contenance.

L'âge entre dans la constitution du matériel d'exploitation comme second élément. C'est la succession non interrompue des âges, suivant une échelle ascendante de un à cent ringt ans, qui permet seule de produire, tous les ans, des bois de cent vingt ans. Les conditions du travail et les habitudes de la vie amenant la reproduction des mèmes besoins tous les ans, l'aménagement doit tendre à livrer les mèmes produils à la consommation chaque année. Quand on dit: les mèmes produits, il s'agit éridemment d'une approximation que comporte la nature mème de la production. La gradation des âges peut être considérée comme acquise lorsqu'il existe entre eux des écarts de 5, 10 années ct nème plus selon les cas. Pour les petites propriétés, l'aménagement peut tendre à fournir des coupes annales ou biennales et à s'affranchir ainsi de l'obligation d'un rerenu annuel qui est la rigle générale des grands propriétaires, comme l'itat et les communes.

Nous arons indiqué l'état de régétation comme le troisieme élément de la conslitution du matériel d'expluitation. La régcitation des arbres croissant ensemble sur un terrain produit ce que les forestiers idjplllent un peuplement, dont la yaleur rarie avec 
chaque àge suivant des lois naturelles dont l'existence est certaine. Malheureusement ces lois de la végétation sont fort peu conrues au point de vue expérimental et nous ne pouvons qu'en indiquer l'existence.

Pour que les peuplements correspondant à chaque âge soient bien constitués relativement à la formation du capital d'exploitation, il faut qu'ils comportent la consistance et le volume que la nature assigne à clıque âge dans des conditions données de végétation. La consistance est relative au nombre des tiges à l'hectare; le volume s'exprime par le nombre de mitres cubes par hectare. Pendant que le premier nombre suit une marche décroissante avec l'âge, l'auire suit une marche ascendante, sans que l'on puisse encore exprimer numériquernent la loi de cette marche.

Le nombre des tiges par hectare débute par être tris-grand des les premiers àges de la vie d'un peuplenent. Un certain nombre périssent étouffées par les plus vigoureuses, et la quantité des tiges va successivement en diminuant au fur et à mesure que leur grosseur s'accroît. C'est sur ce fitit qu'est fondée la pratique des éclaircies, qui sera exposéc au chapitre des fulaies pleines. On coneoit que la connaissance de cette loi de la végétation, ves!-il-dire du nombre des liges par hectare pour chaque essence et pour les diflérentes conditions de sol et de climat, serait une 
précieuse règle pour la conduite de ces opérations délicates qui n'ont aujourd'hui pour direction que l'expérience et le coup d'œil des forestiers.

Ia figure ci-jointe donne une idée de cette loi de décroissance du nombre des tiges à l'hectare.

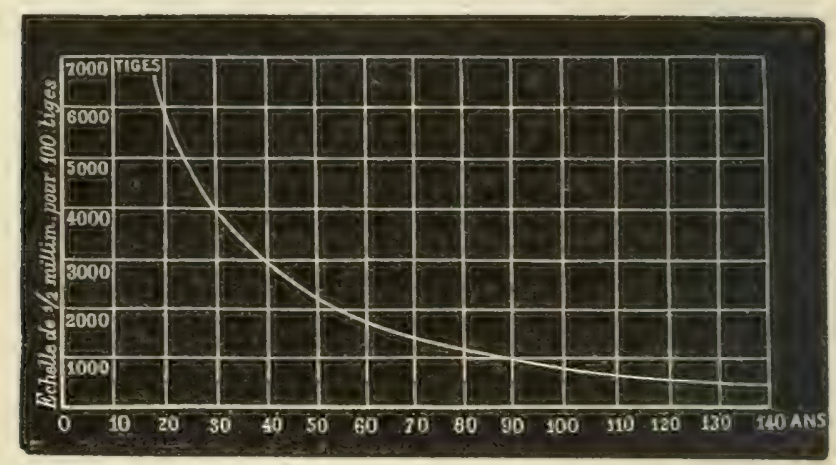

Fiğ. 1. - Décroissance du nombre des tiges à l'hectare.

Sur une ligne droite divisée en parties égules corrispondant à chaque dizaine d'années, on a élevé des perpendiculaires représentant, à raison de un demimillimètre par cent tiges, le nombre de tiges à l'hectare. De cette façon, en appliquant sur la courbe une règle dirisée en millimètres, on peut aroir une idée du nombre de tiges à l'hectare que doit présenter un peuplement à chaque âge. La courbe a été construite pour des peuplements d'essences mélangées de hêtre et de sapin; il est érident qu'elle varie arec chaque essence et arec les conditions de la régétation; aussi no doit-elle être considérée que comme un simple 
renseignement destiné à appeler l'attention sur ce point encore peu expérimenté de la constitution des peuplements.

Quand un hectare de forêt a vécu de sa vie normale et régulière, sans accident et sans trouble dans sa végétation, il doit présenter un volume de bois spécial à chaque àge, à chaque essence et variant arec le climat et la situation du lieu. Gette échelle

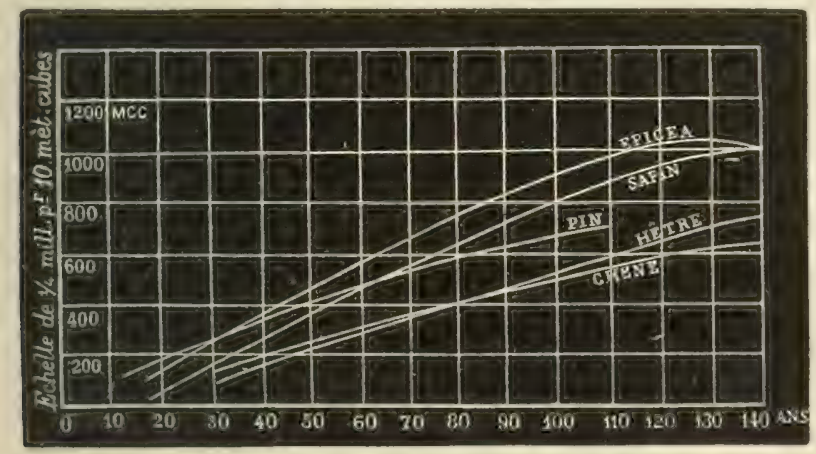

Fig. 2. - Courbe des volumes à l'hectare.

des volumes correspondant à chaque âge constitue une seconde loi de la végétation, loi encore inconnue et qui ne nous sera révélée que par des expériences nombreuses faites pour toutes les cssences et dans chaque localité. La firgure ci-jointe présente un essai de construction de celte courbe d'apris des tables publiées par les administrations allemandes pour cinq essences principales.

Les volumes sont représentés par des perpendicu- 
laires èlevées à chaque division d'âges et d'une longueur calculée à raison d'un quart de millimètre par dix mètres cubes. La courbe donne ainsi les volumes de l'hectare à chaque catégorie d'àges; mais le lecteur n'y doit voir qu'un simple renseignement, car les expériences sont loin d'ètre assez nombreuses et assez concluantes pour permettre de construire, mème d'une manière approximative, une courbe de la végétation.

La connaissance de cette courbe serait fort utile non-seulement pour bien régler la constitution du matériel d'exploitation, mais encore pour rèsoudre les questions les plus importantes de l'économie forestière; celles, par exemple, de savoir à quel âge il faut régler l'exploitation d'une forêt pour aroir la plus grande quantilé de produits en matière eu égard au capital engagé, celle de la valeur de ce capital, etc.

Dans l'état actuel, les forestiers se borrent à exprimerl'état des peuplements par des noms particuliers: jeunes semis, fourrés, gaulis, perchis, hauts perchis, jeune futaie, futaie pour les forèts traitées en futaie pleine; recrus, jeunes taillis, taillis, vieux taillis pour les forêts traitées en taillis. Ces noms n'expriment que l'état des peuplements par rapport à la grosseur des tiges et n'indiquent rien quant au nombre des tiges à l'hectare et à leur volume. Celte 
condition importante s'exprime par des adjectifs: clair, serré, complet, etc. ${ }^{\prime}$

Quoi qu'il en soit, l'état des peuplements à raison de leur végétation dépend toujours d'un élément principal: l'âge; et quand nous arons dit que le matéricl d'une exploitation de forêt à cent vingt ans comporte une échelle d'âges graduée de un à cent vingt ans, nous arons, par une formule synthétique, entendu que l'on doit aroir une suite de peuplements correspondant à des âges gradués de un à cent ringt ans. Il était nécessaire cependant de l'expliquer pour bien en indiquer le sens et la portée.

Explications. - Nous sommes en mesure maintenant de revenir sur la définition générale de l'aménagement et d'en peser exactement les termes.

1. Quant j'ai dit que le premier but de l'aménagement était de déterminer exactement le matériel d'exploitalion, il ne faut pas croire qu'on doive l'éraluer en volume ou en argent. Rien de tout cela n'est utile. Ce matériel doit être, pendant tout le temps de l'exploitation, invariable et constant, c'esi-ḋ-dire offrir toujours dans une même proportion les éléments d'âges, de contenance et de peuplement qui le constituent. On évaluera bien quelques parties en contenance et en volume pour rẻgler les quantités à

1.M. Bagneris, Manuel de sylviculture, 1873, p. 5. 
cxploiter; mais il suffira, pour déterminer le matériel lui-même, de le délimiter et de le régler de telle sorte que les coupes ne puissent jamais l'entamer. Les combinaisons d'aménagement devront être concues de manière que la végétation de chaque année répare les quantités exploitées.

$2^{\circ}$ Celui qui entreprend une exploitation forestière doit, comme celui qui est à la tête d'une exploitation agricole, se proposer un but. Il peut vouloir produire une marchandise déterrninée: des étais de mines, des perches à houblon, des planches, des charpentes ou de la simple charbonnette. Il peut ne pas s'inquiéter de la nature des produits et n'avoir en vue que la plus grande somme d'argent qu'il retirera de son immeuble relativement au capital engagé. Il peut aussi, s'il n'a souci que des intérêts du consommateur, chercher à produire la plus grande quantité de bois possible ou les marchandises les plus utiles. Ces différents buts sont autant de bases d'exploitabilité $^{1}$; ils correspondent à autant d’àges fixés pour l'exploitation d'une forêt. Je n'entrerai pas dans le détail des moyens que l'on emploie pour les déterminer; cela sortirait du cadre et du but de ce livre, mais on voit que toute recherche d'exploitabilité aboutit toujours à la fixation de l'àge auquel on veut

1 Le terme d'exploitabilité désigne l'âge auquel un propriétaire doit couper ses bois pour satisfaire à un but déterminé. 
soumettre la révolution d'une foret ${ }^{4}$. Or l'importance, la valeur du capital d'exploitation d'une forèt dependra tonjours de l'àge auquel on voudra couper les bois. Si on exploite à trente ans, le matériel sera formé par des peuplements de un à trente ans; si la révolution est de ringt ans, il sera formé par dos bois igés de un à vingt ans. On voit done que les mots a déterminer le matériel dexploitation 》 compremnent implicitement les recherches dexploitabilité, la fixation de la rérolution la plus arantageuse; c est mème la prenière opération à effectuer pour cette détermination ${ }^{2}$.

3. Il semble aussi que la définition derrait relater un préliminaire à effectuer arant de chercher à déterminer ce matériel : la fixation du régime, taillis ou futaie, et parmi ceux-ci, du mode de traitement: futaie pleine, futaie jardinée, taillis simple, daillis composé etr., auquel la forèt sera soumise. Femarquez bien que, la plupart du temps, le régime, le mode de traitement sont acquis, commandés par le nature des essences et la position du propriétaire. Du moment où il est possible de soumettre un

1 La récoutsa est le temps perdant lequel l'expl witation d'une forêt la

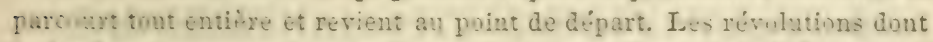
Ia durit ne correspond pas an twane chisi pour l'expl itabilité pronent

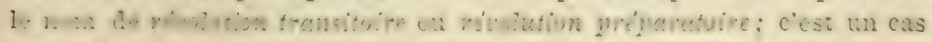

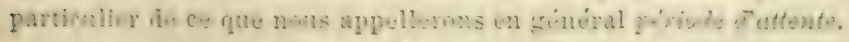

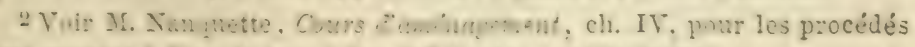
qui strent is tixer le tume des diftentes expluinbilites. 
taillis ou une futaie à un grand nombre de révolulions, c'est parmi ces révolutions que le propriétaire fera son choix et qu’il doit déterminer le matériel d'exploitation correspondant. Telle est la généralité des cas; on ne transforme pas du jour au lendemain le traitement d'une forêt comme on change le régime d'une usine. Ces longues transformations, que nous étudierons au chapitre des conversions, ne sont entreprises que par des propriétaires exceptionnels, l'État et les communes, dans des circonstances déterminées. Mème dans ces cas particuliers, la fixation du régime est bien plus un choix à faire, une determination à prendre, qu'une opération à effectuer. La généralité des termes « déterminer le matériel d'exploitation » comprend donc tous les cas, puisque les différents régimes et modes de traitement des forèts ont pour caractère précisément un matériel spécial d'exploitation, grand pour les futaies, beaucoup plus faible pour les taillis.

L'opération de l'aménagement, c'est-à-dire la détermination du matériel, entraine done le choix du régirne et du traitement comme elle entraîne le choix de la révolution.

$4^{0}$ Le choix du terme d'exploitabilité est intimement lié à la nature des propriétaires. Le particulier ne s'occupe des intérêts de la consommation qu'autant que ces intérêts le touchent et lui assurent un 
dibucuché arantageux. Les communes, et l'État plus encore que les communes, représentent en quelque sorte le consommateur dont les intérêts se personnifient dans ces ètres moraux. La formule de la définition qui consiste à désigner en général l'avantage, les conrenances du propriétaire, préroit donc toutes les situations.

$5^{n}$ La définition assigne deux buts à l'opération de l'aménagement: la détermination du capital d'exploitation et le règlement des coupes qui sont l'expression de la production annuelle. Nous verrons toujours ce double but se manifester, par exemple par la confection d'un plan général de l'exploitation et par celle d'un plan spécial des coupes. L'un tend a modeler la forèt suivant le but que l'exploitant s'est proposé. Il scra immuable autant que peurent l'ètre les buts proposés et les œurres humaines. L'autre regle spécialement les coupes à faire pour une courte période d'années. Ce serait anticiper sur les chapitres suivants si nous insistions sur ce point, que nous ne faisons qu'indiquer.

$6^{\circ}$ L'aménagement n'est en réalité que l'application à un hut déterminé des riogles culturales. Sans leur observation il n'y a point de bon plan d'exploitation possible, comme en agriculture l'assolement le plus avantageux, les bàtiments les mieux aménagéx ne peuvent remplir leur but qu'à la condition d'oh- 
server les règles agricoles commandées par la nature du terrain et l'espèce des produits cultivés. La définition retrace cette alliance intime de la sylviculture arec la conduite de l'exploitation forestière en chargeant l'aménagiste de règler la nature, l'ordre, la marche des coupes. Elle le rappelle ainsi à la stricte observation des principes culturaux, des regles d'assiette et des lois de la physiologie régétale.

Le lecteur voudra bien excuser cette longue paraphrase d'une définition que je me suis efforcé de rendre aussi claire que possible. Dans un lirre où quelques pages seulement sont consacrées à la théorie, ce n'est qu'autour de la définition que peuvent graviter les notions élénentaires destinées à éclairer les explications pratiques ${ }^{1}$

Utilité. - Certains propriétaires de forêts ont prétendu que l'aménagement des forèts est pour la propriété privée une opération illusoire, n'ayant aucune utilité, attendu que le morcellement inévilable des fortunes en compromettra toujours ie sort. Ces propritaires ont confondu l'utilité d'un aménage-

1 Qu'il mous suit permis de faire remarquer que la définition que nous avous adoptée se confond ainsi avec celle beancoup plus concise du Cours Taménagement: "Lne opération qui consiste à régler pour une ou plusieurs révolutions le mode de culture et l'exploitation d'une forêt dans le plus grand intéêt du propriétaire et de la consommation." Dans un livre concernant l'administration d'un domaine forestier, il était nécessaire d'insister sur le capital d'exploitation, car il ne peut y aroir de bonne administration, de gestion raisonnée, sans une comptabilité, at celle-ci ne peut se concevoir saas l'étahlissement d'un compte de capital. 
ment avec ses chances de durée. Aucune œuvre, aucun projet ne peuvent s'affranchir des éventualités de 'arenir; celui qui aménage sa forèt est soumis à cette loi comme celui qui fonde une usine ou une société commerciale. Il n'est pas bien certain que les aménagements des forèts domaniales et communales ne ccront pas tout aussi exposés à n'èire point suivis que ceux des particuliers.

Autre chose est l'utilité de l'aménagement: à cause de la régularité qu’il donne aux exploitations, à cause de la facilité qu'il fournit pour exécuter les travaux orestiers utilenent et sans perte de temps, l'améaggement imprime à une forêt particulière un caracère de bonne gestion qui en augmente la valeur, comme en agriculture toute ferme bien réglée, bien comloincé, aura toujours, à ètendue égale, plus de valeur cqu'une terre mal soignée, mal dirigée.

N'existe-t-il pas d'ailleurs un grand nombre de circonstances daus lesquelles l'aménagement doit ètre suivi ou peut ètre ordonné? Nos lois civiles l'imposent à l'usufruitier, et très-nombreuses sont les situaions usufruitieres: jouissance légale des parents sur cs bicns des enfants, communauté des époux, droits les conjoints mariés sous certains régimes, détenion de bomne foi, envoi en possession, etc., voilà utant de situations daus lesquelles la loi impose à 'usufruiticr l'obligation de respecter le matériel 
d'exploitation d'une forèt exprimé par la formule juridique de la substance de la chose ${ }^{1}$. Combien de contestations ruineuses auraient été évitées dans des familles, si le chef avait, cn vue précisément de cet arenir ignoré, fiit aménager la forèt reçue en dot ou en succession! Que de liquidations seraient rendues faciles par un aménagement et une comptabilité forestière permettant de classer immédiatement ce qui est revenu et ce qui est capital, ce qui tombe en usufruit, ce qui est sujet à reprise. Que de puissantes familles auraient ignoré la désunion!

États des forêts. - Nous arons indiqué quelle succession de peuplements d'àges gradués et d'état identique de régétation doit présenter une exploita. tion de forêt. G'est cette conception théorique, 'que les forestiers ont appelée forèt normale ${ }^{2}$, qui n'existe jamais dans la nature, mais que l'on doit aroir toujours présente à l'esprit, non pour modèle à atteindre (cela est bien impossible!), mais pour base, pour raison explicative de toute combinaison d'aménagement. Le matériel d'exploitation est constitué quand les trois élérrients, contenance, àge, élat de végéta-

1 Voir Code civil, art. 578 et suiv.

2 La forêt normale est celle qui présente une succession d'âges gradués et de peuplements correspondant ì ces âges et régétant dans des conditions identiques de contenance, de sol, de climat et de situation, telle qu'elle serait produite par une rigginération régulière, conduite pendant toute la durée d'une révolution. 
tion, existent au complet d'une manière suffisante dans la forèt à aménagecr. Il peut done yavoir des bois dans lesquels le capital est constitué quant à la gradation des âges, et qui ne présentent pour chaque îge qu'une contenance ou un volume insuffisant. On peut ainsi aroir affaire à bien des cas différents, dont les circonstances particulières de la régétation dans chaque climat rendent le nombre presque infini; mais chacun de ces cas peut se ramener à l'un des trois suivants qui seront la base de notre travail :

1o Le capital d'exploitation est constitué relatiinchent au terme choisi pour l'exploitatilite;

2o Ou il est suraboindant;

$3^{\circ}$ Ou il est insuffisant.

Le premier cas est celui d'une forèt dont les éléments existent dans une proportion suffisammentcomplète pour satisfaire au but que le propriétaire a en vue. On l'appelle aussi forèt régulière, pour indiquer cqu'clle se rapproche de l'idée théorique de la forèt normale, mais autant que le comporte, bien entendu, la nature des choses.

Le second cas est malheureusement rare. Les jesoins incessants de la vie ne permettent de le rencontrer que d'une manière exeptionnelle. Il se présente cependant lorsqu'un propriétaire veut abaisser la révolution à laquelle sa forèt a été jusqu'alors soumise. 
Le troisieme est de beaucoup le plus fréquent; c'est celui qu'un administrateur soucieux de l'avenir doit tendre à reconstituer é à améliorer.

Pour chacun de ces cas, le lecteur doit bien se persuader que l'on n'aménage en réalité que des forêts plus ou moins irrégulières.

Parcellaire. - La difficulté est de s'assurer par un moyen pratique de la manière dont est constituée la forêt que l'on veut aménagger. On comprend facilement que, dans une forêt un peu étendue, on ne peut jamais avoir, à première vue, une idée bien nette et bien exacte de ses ressources; il faut donc commencer par faire une analyse et un inventaire de la forêt. Mais il ne s'agit pas ici d'un inventaire en nombre d'arbres ou en mètres cubes, il s'agit de quelque chose de beaucoup plus simple: on parcourt la forèt, en séparant par des lignes, et en les arpentant, les parlies qui, étant homogènes par l’àge, la nature des essences et la végétation, sont susceptibles d'un même traitement. Ces morceaux de forìt, que l'on appelle des parcelles, se gravent facilement dans la mémoire et permettent de saisir parfaitement l'ensemble des ressources de la forèt; on devra s'attacher d'ailleurs à séparer les parties dans lesquelles l'exposition ou la nature des essences imprimeront d'une manière à peu près permanente un cachet spécial à la culture des peuplements. On saura 
DE LAMÉNAGEMEXT EX GÉXERAL.

ainsi, par un trarail de mémoire et d'expérience, si le capital d'exploitation est constitué, c'est-à-dire si la forêt présente un matériel suffisant pour permettre une exploitation aỹant pour base la rérolution qui a ẻté adoptée.

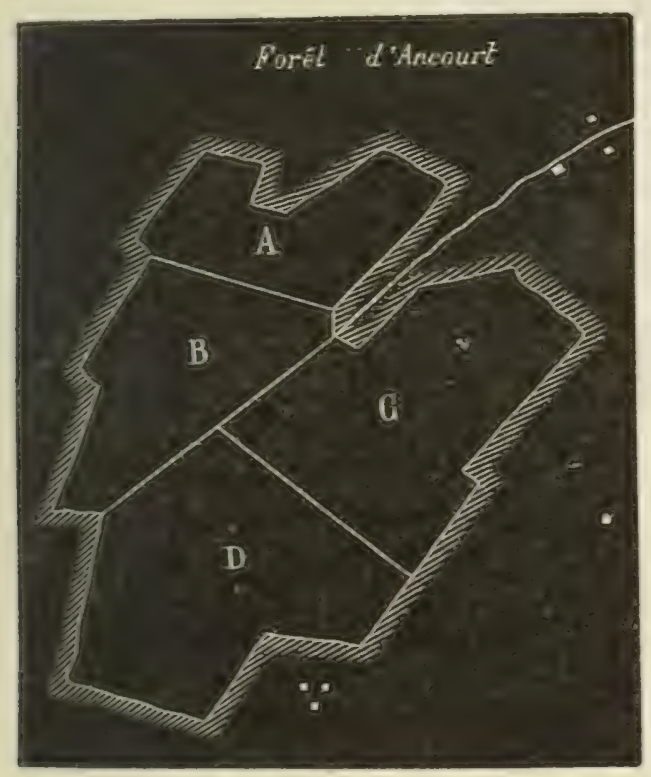

Fig. 3. - Parcellaire.

Chacume des parcelles est soigneusement décrite dans un calier auquel on peut toujours se reporter. Mais, dis que la forèt est un peu grande et dès que le nombre des parcelles devient considérable, la confusion ne tarde pas à naitre dans l'esprit, et il est 
nécessarre de l'éclairer par des procédés supplémentaires.

Les uns dressent un tableau synoptique des parcelles, dans lequel les éléments principaux : àge, peuplement, régétation, etc., sont résumés; ils présentent ainsi à l'esprit les parcelles de la forêt sous une forme en quelque sorte condensée.

Les autres s'aident de procédés graphiques dont ies systimes sont multiples et dont voici un spécimen.

Sur une ligne divisée en parties igales correspondant aux contenances, on construit des rectangles dont la base exprime la contenance, et la hauteur l'àge des peuplements. La ligne supéricure de ce rectangle indique, si elle est droite ou sinueuse, la régularité ou l'irrégularité du peuplement. Sa consistance est représentée par des hachures plus ou moins serrées; un signe cn forme de $\Lambda$ marque, selon son écartement, l'activité de la végétation. S'il y a des gros arbres, une ligne dont la hauteur est égale à lcur âge les représente; et enfin un, deux ou trois points indiquent si ces arbres sont plus ou moins nombreux dans le peuplement. En outre, les essences et le sol s'expriment par des couleurs conrentionnelles sur la carte topographique de la forêt. 
DE LAMFNAGEMENT EN GENERAL.

La ficrure ci-dessous exprime immédiatement à

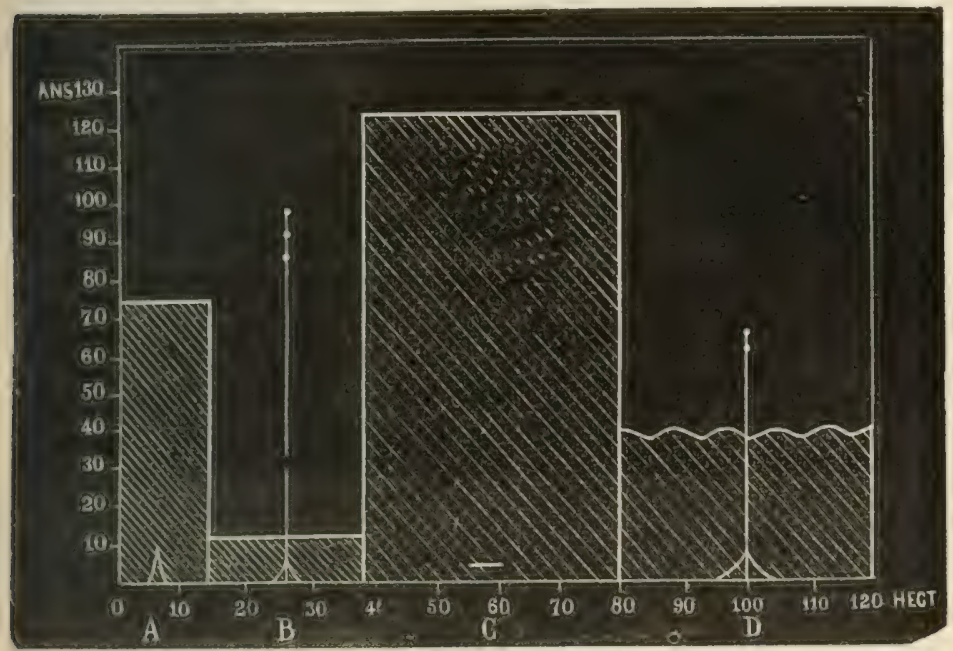

Fig. 4, - Représentation graphique des parcelles

l'ocil, dans ce système de représentation graphique, les peuplements suivants:

A. 14 hect. haut perchis passant à l'état de jeune futaie de 75 à 80 ans, d'une végétation très-active; peuplement très-dense et régulicr.

B. 24 lioct. gaulis et fourrés, réguliers, bien venants; végétation ordinaire; très-complets, surmontés de grands arbres en assez grand nombre; âges moyens : 10 ans pour le peuplement, 90 it 100 ans pour les arbres.

C. 41 hect. futaic exploitable de 120 à 130 ans, très-claire, préscntant même quelques clairières; végétation ralentie, stationuaire; peuplement régulier dans son ensemble. 
D. 41 hect. perchis de 40 ans, en moyenne, mélangé de tiges plus ou moins jeunes, formant, arec des arbres de 70 it 75 ans, assez nombreux, un massif irrégulier et clair; régétation assez peu active.

En combinant ces parcelles représentées à l'aide de petits cartons découpés, en appliquant ceux-ci sur un tableau construit suivant les mêmes dimensions graphiques et sur lequel on a tracé la ligne ascendante de la gradation des àges, on voit que ce matériel peut passer pour constitué.

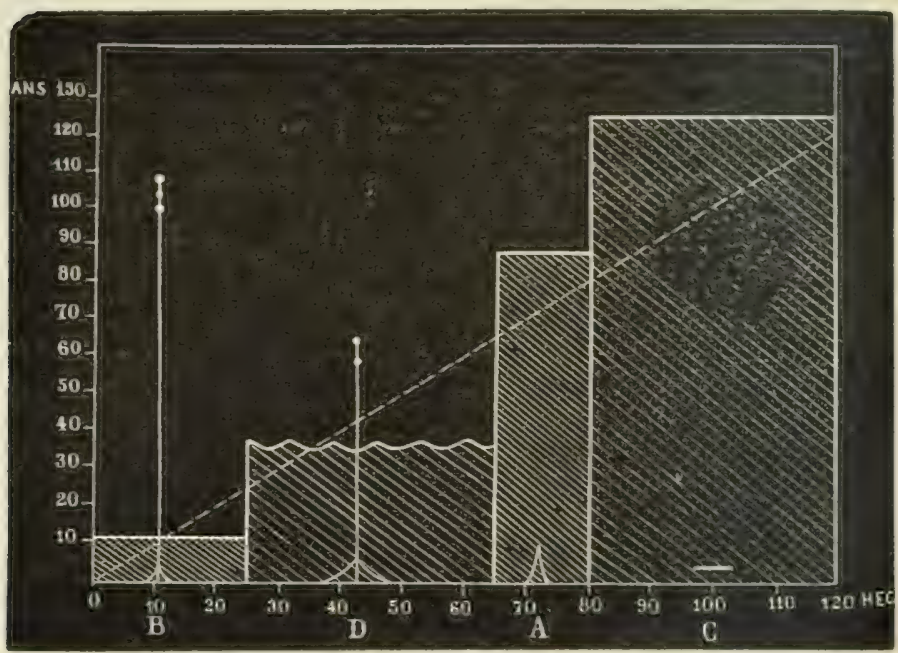

Fig. 5. - Combinaison d'aménagement.

En comparant enfin la combinaison exprimée dans la figure ci-dessus avec le plan de la forêt, on voit si ce matériel est régulièrement disposé, tant au point 
de rue d'une succession convenable des coupes qu'au point de vue de l'observation des règles culturales.

Tel est le mécanisme des procédés que l'on peut employer. La formation des parcelles a ses règles et. ses théories, dans l'examen desquelles je n'ai point à entrer, parce que ce lirre a pour but d'apprendre, non à faire, mais en quoi consiste un aménagement, et à expliquer comment cette opération n'est pas autre chose qu'une base essentielle de la conduite, de la gestion et de la comptabilité d'une exploitation forestière. Rappelons seulement que pour faire un bon parcellaire, l'aptitnde et l'expérience sont nécessaires au forestier comnse elles le sont à l'agricultem pour faire une bonne élude des parcelles de terre qui doivent entrer dans la combinaison des assolements. 


\section{DEUXIÈME PARTIE.}

\section{Aménagement des forêts à petit capital d'exploitation.}

\section{TAILLIS.}

\section{GHAPITRE PREMIER.}

TAILLIS SIMPLES.

\section{$\S 1$.}

Taillis dont le matériel d'exploitation est constitué.

Rien n'est facile à comprendre comme l'aménagement des taillis; j'entrerai cependant dans quelques détails à leur égard, parce que, la simplicité étant la qualité la plus essentielle d'un aménagement, leur formule servira de modèle et de guide pour celui des futaies.

Nos 120 hectares de bois à traiter en taillis à vingt uns peuvent présenter:

$$
\begin{aligned}
& 6 \text { hectares de } 1 \text { an; } \\
& 6 \quad \text { " de } 2 \text { ans; } \\
& 6 \quad \text { " de } 3 \text { ans; } \\
& \ldots . . .
\end{aligned}
$$

présenlant les mêmes conditions de végétation, se suivant les uns les autres d'une manière régulière 
(c'est la forèt régulière), ou bien disposés d'une maniere irrégulière et mélangés sans ordre. Ce dernier cas, qui est assez rare dans les taillis ou les exploiłations se font généralement de proche en proche, cst trés-fréruent dans les futaies; et il sera beaucoup mieux compris après l'explication de l'aménagecnent des taillis dont le capital est constitué d'une manière rẻgulière.

\section{A.}

Aminaggenent des taillis dont le matériel d'exploitation cst constitu!é et régullièrement disposé.

Il ne faut pas prendre à la lettre l'idée que je vicns de domner l’une forèt régulière; si, pour réunir cette qualité, il fillait trourer, dans l'exemple qui nous occupe, une succession de parcelles de 6 hectares de un, deux, trois... vingt ans, se suivant les unes au bont des autres, il est fort probable que jamais on ne rencontrerait unc pareille perfection. Une forèt parse pour irguliere quand elle présente tune échelle diages suflisimment et assez régulierement dispone pour que le matériel d'exploitation correspondant at l'exploital)ilité que l'on a choisie soit constitui regulierment. Il y a en sylviculture comme en agriculture des limites et des approximatims qu'il fut sitsoir comprendre et admettre.

Plan général d'exploitation. - xuppo:ous que le 
parcellaire qui se fait le plus souvent dans les taillis, cn relevant les lignes des exploitations précédentes, ait donné :

A. 12 hectares taillis, chêne et charme. - 20 ans.

B. 6 " " in̉. . . 17"

C. 18 " "chêne pur . . . 15 "

D. $15 "$ id. . . . . $12 "$

E. 9 " " chêne, hêtre, bouleau. 11 "

F. 12 " $"$ id. 9 "

G. 24 "jeunes taillis, hêtre et charme . 6 "

H. 6 " " hêtre et aulne. 4 "

I. 19 " plantation, chêne et charme . 3 "

120 hectares.

On divisera tout simplement les 120 hectares en ringt coupes de 6 hectares chacune, se suivant de proche en proche, et chaque coupe sera affectie à chacune des années de la rérolution. On admet généralement dans les taillis que les coupes égales donneront des produits égaux. Il n'est pas bien utile de chercher à faire des coupes ayant des contenances proportionnelles à la fertilité du terrain, car cette fertilité est fort difficile à mesurer d'une manière exacte. Si, malgré cette difficulté, on s'était attachéà donner aux coupes des contenances proportionnelles à la fertilité, il pourrait bien arriver qu'à la rérolution suivante les conditions de la fertilité vinssent à changer, de sorte que le travail qui a consisté à diviser la forèt en coupes, à les arpenter, à les aborner, et à défricher leurs lignes, serait à recommencer. 
Les mesures les plus simples sont les meilleures, et

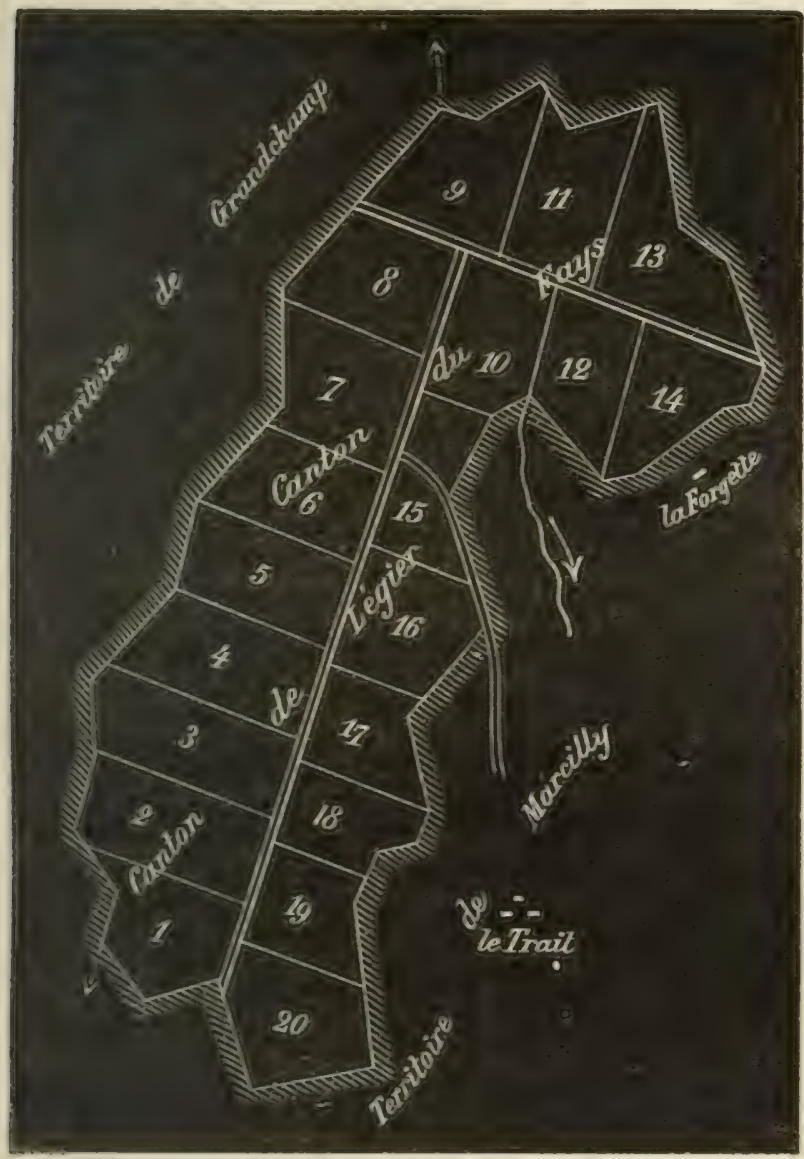

F:: 6. - Aménagement on taillis.

rien nimpeche, sil se trouve dans une coupe un rochrr ou un ride impropre a la végétation ru bois, d'anguncuter d'aulant la contenance de cetle coupe. 
L'aménagement, que les anciens foresticrs appelaient la mise en régle ou mise en contres réglées, est pour les taillis quclque chose de si simple qu'on se contente généralenent de faire un plan portant les numéros des coupes, sans mème y indiquer les parcelles qui ont servi à faire l'analyse de la forèt.

A ce plan est annexé un tableau des coupes et de leur contenance. Ce tableau forme le plan général de l'exploitation, et si l'anienaģement a été fait en 1860 , il est dressé ainsi qu’il suit.

IPlan général d'exploitation (1860-1879).

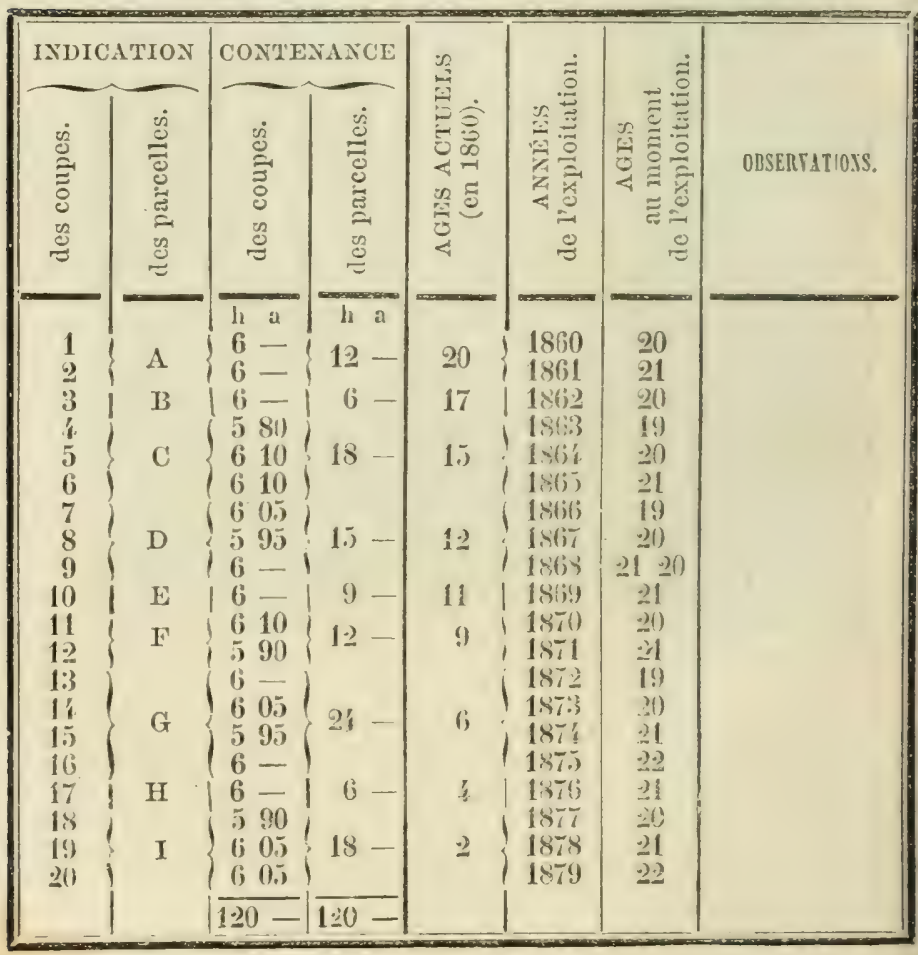


A cela se horne le mécanisme de l'aménagement les taillis; mais, pour bien faire comprendre l'améagement des futaies et pour leur appliquer la sim-licité qui fait le mérite des aménagements de taillis, lous entrerons dans quelques détails supplémenaires.

\section{Du compte d'aménagement. - L'aménagement} erait terminé, s'il n'était de la plus grande utilité le prescrire certaines mesures pour en surveiller a marche. Dans une exploitation de forèt, comme lans toute affaire agricole ou industrielle, il est le la plus incontestable utilité d'avoir une compabilité. Sans doute, l'exploitant aura toujours des irres pour le salaire des ourriers et pour la vente les produits, mais ce n'est pas de cette compabilité que je veux parler, c'est uniquement du compte capital. Depuis quelques années, la comptaoilité a fourni à l'agriculture l'aide puissant de la 'égularité et du contrôle, et tous les auteurs qui ont crit sur ce sujet ont surtout insisté sur le compte capital agricole. Dans une exploitation agricole, le compte capital varie par les réparations des bâtinents, l'usure de l'outillage, la mortalité du bétail, atc., elc. S'il n'en tenait pas un compte exact, le ultivateur ne saurait jamais ce qu'il gagne, et il est némc nécessaire de prélever tous les ans sur les roduits une certaine somme pour maintenir tou- 
jour's le capital à une valeur marchande et réelle. Ce que j’ai lit du matériel d'exploitation d'une forêt fait suffisamment comprendre qu'en sylviculture rien de pareil ne se passe: le matériel d'exploitation n'est pas exposé à de pareilles causes de dépérissement et il ne saurait y aroir lieu à une comptabilité aussi compliquée. Le registre de contròle de l'aménagement ne derra donc indiquer qu'une scule chose: savoir si, à un moment donné, on n'a pas entamé le capital.

Rien n'est simple comme ce compte de capital forestier.

Il ne faut pas vouloir en faire un registre destiné à rérifier les procédés d'estimation et de cubage, ni un compte moral et administratif, ni un document pour suirre les prix et l'état du marché. Tous ces renseignements, qui ont leur utilité, peurent faire l'objet de livres auxiliaires, comme ceux destinés aux comptes des salaires, des traraux et des rentes. Si on voulait consigner tous ces documents dans le compte d'aménagement, on arriverait à construire des etats compliqués dont la nultiplicité des colonnes effraie l'oeil et ne fournit à l'esprit que des ressources confuses. Le caractere d'une bomne comptabilité est d'ètre très-simple et d'aroir un but bien nettement déterminé. 11 fant qu à un moment donné le propriélaire ou le rérificateur de la gestion puisse saroir 
si l'on suit la marche de l'aménagement et de quelle manière il a été appliqué. C'est surtout dans les gestions de forêts importantes que le compte doit conserver son cachet de simplicité pour bien satisfaire à son but: le contrôle de l'aménagement. Dans les taillis, on le tient mème peu souvent; mais c'est un tor't, car on oublie le moment où la première coupe a été faite; on effectue sourent, quand le besoin s'cn fait sentir, des coupas par anticipation; on en retarde d'autres, et il arrive qu'au bout de quelques années on ne sait plus où l'on en est. Je donnerai ci-après le morlele de ce registre, qui se borne à une simple page à tenir en tète des comples auxiliaires de l'exploitation; mais auparavant je dois parler d'une pratique fort avantageuse à laquelle se prète très-bien la gestion des forèts et qui est très-utile pour les particuliers et les communes.

De la réserve. - Il est dans les règles de la plus habituclle prudence de ne pas toujours et tous les ans dépenser la totalité de son revenu. Des besoins imprévus, des circonstances de mille natures déterminent tout proprictaire à se constituer une réserve. Dans l'iudustrie, dans l'agriculture, on ne peut former cette réserve qu'en playant tous les ans quelque chose prélevé sur les produits. Le plus souvent es plarements ne sont que l'entrée dans une nouvelle aflinire, soit industriclle, soit agricole; toujours 
les placements destinés à une réserve demandent une administration et des soins. Les propriétaires de forèts n'ont qu'à laisser sur pied une partie de leurs revenus, et la nature se charge toute seule d'administrer une réserve qui jouit ainsi de la plus grande solidité et de l'avantage de produire elle-même ses intérêts.

Il y a dans les forêts deux manières d'établir cette réserve.

$1^{0}$ Réserve à assiette fixe. - Le premier mode consiste à séparer sur le terrain la quantité d'hectares que l'on veut garder pour les besoins imprévus, $10,20,250 / 0$, si l'on vent affecter à la réserve $10,20,250 / 0$ de la production. Si nous admettons, comme cela se fait dans les forêts communales, un quart pour la réserve, il n'y aura plus, dans la forèt que nous avons prise pour exemple, que quatre-vingt dix hectares pour les coupes annuelles, qui seront ainsi réduites à 4 hectares 50 ares par an, et 30 hectares seront affectés aux besoins imprévus. Le compte d'aménagement sera tenu de la façon suivante: 
muler au delà de 4 ans, et il y a alors deux manieres de procéder:

Si l'on reut que les coupes de réserve soient égales aux coupes ordinaires, - et cela est sourent utile dans les forèts communales, - on réalise la réserre tous les trois ans; on destine chaque groupe de 3 coupes à fournir quatre coupons qu'on exploite en 3 ans; les trois premiers représentent l'affouage, et le quatrième la réserve.

Si, au contraire, ou veut donner plus d'importance aux produits de la réserve, on ne les réalise que tous les quatre ans; ils sont d'une coupe entière de 6 hectares, et les affouages de 4 hectares 50 ares. Tous les quatre ans, on divise, par un arpentage très-facile, un groupe de trois coupes en quatre coupons, qui représentent chacun la production, distraction faite de la réserve; la quatrième année, on exploite à la fois le quatrième coupon et la coupe cntière qui le suit immédiatement et qui forme la réserve.

Le compte de l'aménagement de la forèt qui nous sert d'exemple est tenu ci-dessous pour cette dernière hypothèse. On doit aroir soin d'inscrire à l'encre rouge les coupes de réserve. 


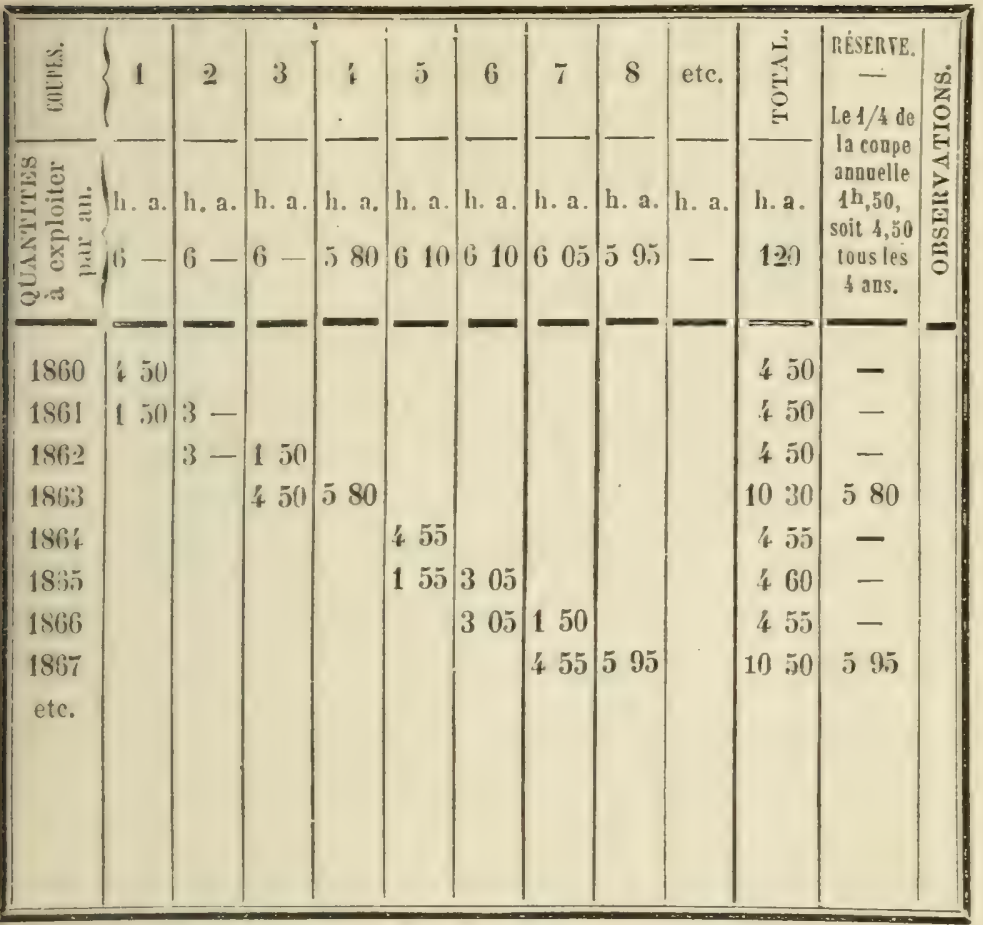

Ce compte montre qu'en 1861 la coupe ordinaire a été formée de $1^{\mathrm{h}}, 50$ pris dans le no 1 et complétée par 3 hectares du no 2 ; cn 1862, elle a été forméc par la moitié du no 2 et $1^{\mathrm{h}}, 50$ du no 3 ; en 1863 , le restant du no 3 a fourni la coupe ordinaire, et le $n^{0} 4$, en entier, a foumi la coupe de réserre, ete.

Ce mode de réserve molile a pour arantage de fommir constamment à la réserve des bois exploitab) le, tandis que, dans une réserre assise sur le terrain, il faut attendre, aprés clıaque exploitation, que lia nalure les ait reconstitucs. Il est, à tous émords, le plus avantageux pour les futaies - mais, daus les 
taillis, oủ la plus légère différence d’àge se fait sentir sur la production, il a pour inconvénient de ne rendre la réserve disponible que tous les quatre ans. Si on la laisse se reporter plus loin, ou si on la devance; si le propriétaire, voulant jouir d'une année de cherté dans le prix des bois, veut anticiper une coupe ou deux, on arrive bientôt à changer l'exploitabilité des taillis et à établir une confusion dans l'échelle des àges, dont l'avenir peut se ressentir. Aussi, dans les bois feuillus des communes, qui s'exploitent le plus souvent en taillis, la réserve, fixée au quart par une disposition spéciale du Code de 1827, est habituellement formée par un quart de la contenance assis sur le terrain, bien que la loi soit restée muette sur le mode d'assiette.

Dans le but d'éviter les arpentages partiels qu'entraine le mode de réserve mobile qui vient d'ètre exposé, on a quelquefois augmenté d'un tiers le nombre des coupes, pour avoir un quart de la forèt en réserve. Au lieu de 20 coupes de 6 liectares, on a assis sur le terrain 26 coupes de 4 hectares 62 ares; les 6 coupes de réscrve peurent ètre colloquées ensemble dans une partie déterminée de la forèt ou intercalées dans les 20 coupes de l'ordinaire. On concoit que cette combinaison n'est pas autre chose qu'une réserve à assielte fixe qui peut offrir certains avantages ; elle suppose que le propriétaire ne chan- 
gera pas la quantité de la réserve qu'il veut faire, comme cela arrive pour les communes, oủ cette quotité est fixée par une disposition de la loi.

\section{B.}

Aménagement des taillis dont le matériel est constıtue, mais irrégulièrement disposé.

Il y a fort peu de chose à dire sur l'aménagement des taillis dont le capital n'est pas régulièrement constitué. Au point de vue de la production, si les règles culturales relatives aux abris, à la végétation, etc., ont été olsserrées, il importe peu que les àges se suivent sur le terrain d'une manière régulière; mais, au point de vue des facilités d'administration, de la vente des produits et de bien d'autres circonstances, il est fort avantageux de ramener, autant que possible, les taillis à un état régulier. Quand le capital est constitué, il ne faut pas craindre d'exploiter, même avant leur age, certaines coupes, dans le but de leur faire reprendre leur rang et leur place dans l'ordre futur des exploitations. Le sacrifice que l'on fait sur ces coupes se trourera d'ailleurs compensé par celles qui seront coupées un peu plus tard. Le plan général d'exploitation prend alor's, d'une manière bien plus tranchẻe, le double caractère qu'il doit avoir et qui est: 10 de régler l'ordre et la marche des exploitations pour la résolution dans laquelle 
on entre; $2^{\circ}$ de préparer pour l'arenir et pour les rérolutions futures un matériel d'exploitation com-. plet, régulier et convenablement disposé.

\section{\$ 2.}

Taillis dont le matériel d'exploitation est surabondant.

Quand le matériel d'un taillis est surabondant, c'est-à-dire plus considérable qu'il ne faut pour l'exploitabilité que l'on a choisie, on combine la marche des coupes de manière à pouvoir exploiter deux fois, dans la révolution, les coupes ou parties de coupes qui présentent cette surabondance. Le plan général d'exploitation présentera alors deux natures de produits: 1 o ceux qui dépendent de la marche normale de l'aménagement; $2^{\circ}$ ceux qui sont réalisés en dehors de cette marche, dans le but d'en assurer la régularité.

Supposons, par exemple, que la parcelle I, de 18 hectares, soit âgée, au moment de l'aménagement, de vingl-deux ans; on pourrait l'affecter aux coupes nos 1 , 2 et 3 , et réserver la parcelle $A$ pour les coupes $n^{0:} 4$ et 5 ; mais alors on exploiterait certaines coupes a ringt-cing et ringt-six ans, et comme le propriétaire a adopté vingt ans pour le terme le plus avantageux d'exploitabilité, il est plus fructueux d'exploiter ces 18 hectares dès le début de l'aménağement, pour les couper une seconde fois à la fin de la révolution. 
Le plan général d'exploitation, que l'on complète halbituellement par l'indication sommaire des traraux à exécuter, sera ainsi formé:

Plan général d'exploitation (1860-1879).

\begin{tabular}{|c|c|c|c|c|c|}
\hline 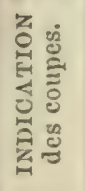 & 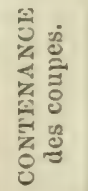 & 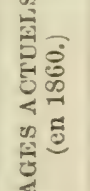 & 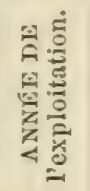 & 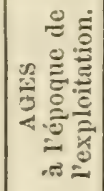 & OBSERVATIONS. \\
\hline \multicolumn{5}{|c|}{$\begin{array}{l}\text { § } \mathrm{I}_{\mathrm{c}} \text { - Produits en dehors de la } \\
\text { marche normale de l'aménagement. }\end{array}$} & $\begin{array}{l}\text { Quantités à exploiter pen- } \\
\text { dant la révolution.... } 138 \text { h. } \\
\text { Quantités à exploi- } \\
\text { ter normalement ....120 }\end{array}$ \\
\hline $\begin{array}{l}18 \\
1 ! 9\end{array}$ & 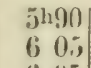 & 22 & 1860 & $\left\{\begin{array}{l}22 \\
2.2\end{array}\right.$ & Différence en plus. . 18 \\
\hline \multicolumn{5}{|c|}{$\begin{array}{l}\text { § II. - Produits dépendant de la } \\
\text { marche normale de l'aménagement. }\end{array}$} & 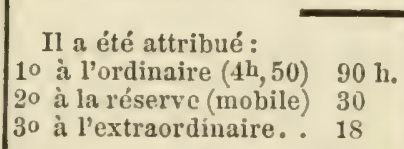 \\
\hline $\mathbf{1}$ & $6-$ & $\begin{array}{l}20 \\
20\end{array}$ & $\begin{array}{l}1860 \\
1861\end{array}$ & $\frac{20}{21}$ & \multirow{18}{*}{$\begin{array}{l}\text { Indication générale des tra- } \\
\text { vaux: } 10 \text { hect. à assainir dans } \\
\text { les nos } 2,4,7,-1 \text { hect. à re- } \\
\text { piquer dans le no } 8 \text {, vers l'an- } \\
\text { née } 1866 \text { - Défricher la laie } \\
\text { sommière sur } 4 \text { métres de large. }\end{array}$} \\
\hline 3 & $6-$ & 17 & 1862 & 20 & \\
\hline 4 & 5811 & 15 & 1863 & 19 & \\
\hline 5 & 610 & 15 & 1864 & 20 & \\
\hline 6 & $\begin{array}{ll}6 & 10\end{array}$ & 15 & 1865 & 21 & \\
\hline 7 & $\begin{array}{lll}6 & 05\end{array}$ & 12 & 1866 & 19 & \\
\hline 8 & 595 & 12 & 1867 & 20 & \\
\hline 9 & $6-$ & $12-11$ & 1868 & $20-21$ & \\
\hline 10 & $6-$ & 11 & 1869 & 21 & \\
\hline 11 & 610 & 9 & 1870 & 20 & \\
\hline 12 & 590 & 9 & 1871 & 21 & \\
\hline 14 & $\begin{array}{lll}6 & \overline{6} & -5\end{array}$ & $\begin{array}{l}6 \\
6\end{array}$ & $\begin{array}{l}1872 \\
1873\end{array}$ & $\begin{array}{l}19 \\
20\end{array}$ & \\
\hline 15) & 595 & 6 & 1874 & 21 & \\
\hline 16 & $6-$ & 6 & 1875 & 22 & \\
\hline 17 & $6-1$ & 4 & 1876 & 21 & \\
\hline 18 & 59 & 1 & 1877 & 19 & \\
\hline $\begin{array}{l}19 \\
20\end{array}$ & $\begin{array}{ll}6 & 05 \\
6 & 05 \\
\end{array}$ & $\begin{array}{l}1 \\
1\end{array}$ & $\begin{array}{l}1878 \\
1879\end{array}$ & $\begin{array}{l}20 \\
21\end{array}$ & \\
\hline & $0-$ & & & & \\
\hline
\end{tabular}




\section{$\S 3$.}

Taillis dont le matériel d'exploitation est insuffisant.

Quand le matériel d'un taillis est insuffisant pour l'expoitabilité que l'on a choisie, l'aménagement se réduit à savoir attendre et à rendre l'attente la moins pénible possible pour le propriétaire:

Supposons que le parcellaire ait donné :

A. 24 hectares chêne et bois blancs . . 14 ans

B. 18 "

id.

C. 24 "

chêne et charme

D. 24 " id.

E. 6 "

id.

F. 24 " vide à repeupler

120 hectares.

On voit, par cette analyse de la forèt, qu'il faut attendre six ans avant de pouvoir exploiter les bois à vingt ans. Cette durée de six ans, qui est l'intervalle qui sépare les bois les plus âgés (tête d'aménagement) de l’àge adopté, a èté appelée période d'attente ou période transitoire.

On a donné, en général, le nom de période d'attente au temps qui est nécessaire, soit pour régulariser une forèt dont le capital est constitué, mais mal disposé, soit pour exploiter la partıe surabondante d'une forèt dont le capital d'exploitation dépasse ce qui est nécessaire, soit enfin pour attendre le mo- 
ment ou l'on pourra commencer la marche normale de l'aménaģement dans une forêt, dont le capital est insuffisant '.

Il y a deux manières de procéder :

La première consiste à établir, dès à présent, les vingt coupes affectées aux ringt années de la révolution, sauf à indiquer ce que l'on fera dans les six années d'attente. (Aménagement défunitif.)

La seconde consiste à régler les exploitations que l'on reut faire dans la période d’attente et à remettre à six ans le moment où l'on fera l'aménagement définitif. (Règlement provisoire d'exploitation.)

Ce dernier mode est généralement abandonné, et ce n'est que dans des cas très-rares, très-exceptionnels, que l'on fait encore des règlements provisoires d'exploitation. Les raisons en sont nombreuses : 1. Dans l'état actuel de nos forèts, dont aucune peutétre n'a la régularité théorique, la première rérolution aura toujours un caractère plus ou moins transitoire. Pourquoi, des lor's, firre une distinction entre les aménagements à période transitoire et ceux à révolution définitive? - 20 On conçoit bien ėgalement que le trarail de parcellaire et d'arpentage est nécessaire pour acquérir la conviction que dans une forèt le matériel d'exploitation est surabondant ou présente des lacunes. Il n'en coûtera donc pas plus

1 Voir la note de la page 31. 
de faire immédiatement l'aménagement, et il serait de mauvaise administration de recommencer le trarail à la fin de la période transitoire. - 30 L'aménagement a non-seulement pour but de tracer la marche de l'exploitation des peuplements existants, mais, en outre', de préparer les massifs qui doirent former plus tard le capital des exploitations futures. Il est donc toujours utile de tracer d'une manière fixe et certaine le plan général d'exploitation.

Enfin, dans le cas particulier où le matériel offre des lacunes et nécessite une période d'attente, il y a encore avantage à faire immédiatement l'assiette de l'aménagement définitif, car on trourera piesque toujours des combinaisons pour rendre au propriétaire l'attente moins pénible, en lui fournissant certains produits ğlanés sur toute l'étendue de la forèt. Si certaines parcelles présentent des arbres à extraire, si d'autres nécessitent des sacrifices d'exploitabilité par leur classement dans le cadre de l'aménagement, c'est dans la période d'attente qu'il sera utile et fructueux de livrer ces produits.

Dans l'aménag̨ement des taillis, la nécessité de l'assictte d'un plan définitif d'exploitation n'a jamais été mise en question; ce n'est que dans les futaies qu'il a ètè fait quelquefois usag̀e de périodes transitoires. si j’en parle maintenant, c'est parce que la simplirite de l'organisation des exploitations de taillis fixe 
mieux dans l'esprit les raisons qui les font abandonner.

Le plan général d'exploitation pour l'exemple dont le parcellaire précède sera dressé ainsi qu'il suit en livrant à l'exploitation une demi-coupe pour chacune des six premières années:

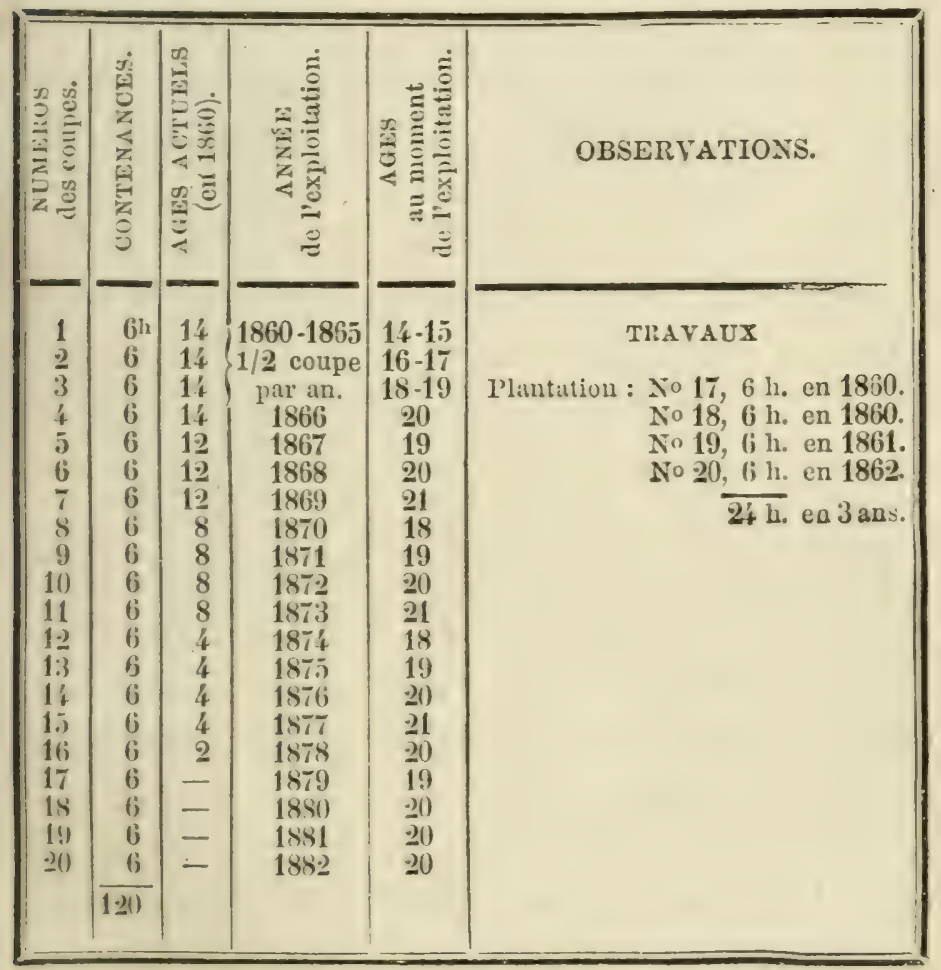




\section{CHAPITRE II.}

\section{DES TAILLIS GOMPOSÉS.}

Les taillis composés ou taillis sous futaie sont ceux dans lesquels le propriétaire élève des arjores destinés à parcourir deux, trois ou quatre révolutions. Leur aménagement se fait entièrement comme celui des taillis simples, mais leur matériel est beaucoup plus considérable, car il se compose :

10 D'une suite de taillis de un à trente ans, par exemple, comme dans le taillis simple;

3. Des arbres de futaie, qui sont plus ou moins nombreux, plus ou moins régulièrement disposés, selon la richesse et les intentions du propriélaire et selon les exigences culturales.

L'aménagement aura pour but de suivre la marche de ces deux éléments du capital d'exploitation. En ce qui concerne le premier, il n'y a nulle difficulté, c'est la marche et l'organisation des aménagements de taillis simples. Pour le second, on concoit très-bien que la présence de cette futaie fait obstacle à la croissance du taillis, et, en mème temps, qu'elle augmente la valeur du capital d'exploitation. Ainsi, selon les essences, la nature du sol et la climat, et selon la richesse du proprietaire, le plan de bulivage, c'est-à-dire le nombre et l'exploitabilité 
des arbres de réserve, doit varier dans chaque forèt et souvent dans chaque coupe d'une même forêt.

Plan de balivage. - Je n'entrerai point dans l'cxamen des procédés employés pour déterminer ce plan de balivage. Ils dépendent du but poursuivi par chaque propriétaire ou, en d'autres termes, de ses convenances et de son intérêt.

si l'on voulait ne tenir compte que du tort causé par les arbres de futaie à la croissance du taillis, on fonderait uniquement le plan de balivage sur les dimensions de chaque catégorie de réserves et sur le couvert que peut supporter un taillis: ce sont des données entièrement expérimentales.

Cette méthode n'envisagerait toutefois la question que d'un côté exclusif, et l'intérêt de chaque propriétaire fait entrer cette donnée en balance avec les avantages qui doivent résulter pour lui de l'éducation d'arbres réservés sur les taillis.

Or, l'écart considérable qui existe entre la valeur d'un brin de taillis et celle que ce brin acquerra 30 ans plus tard fait que le propriétaire n'hésitera pas à laisser croître, après la coupe, un très-grand nombre de baliveaux. Si l'on estime, en effet, à cinquante rentimes la valeur d'un brin de taillis de $30 \mathrm{ans}$, et it trois francs la valeur de ce même brin à 60 ans, on verla que l'argent du propriélaire s'est trouvé plité à un taux supérieur à 5 1/2 p. 100 l'an à in- 
térèts composés. Est-il possible de trouver un plus solide placement, une caisse d'épargne fonclionnant à meilleur's intèrèts?

Le plan de balivage est donc le résultat d'un calcul dont les éléments sont la valeur des arbres et le tort causé au taillis. On conçoit que les solutions de ce calcul sont multiples et dépendent de la nature du propriétaire ${ }^{1}$, de ses besoins actuels et futurs, de la végétation des bois et de lit valeur présumée de leurs produits à raison des exigences du travail el des mojens de transport dans la localité

Toujours est-il qu'il est bon d'avoir un plan de conduite dans ce mode d'exploitation des terres à bois, comme il est convenable d'en avoir un pour l'exploitation du taillis lui-mème.

Le propriétaire d'une forèl de 120 hectares exploités en taillis à 30 ans, à raison de 4 hectares par an, a pu adopter, par exemple, un plan de balivage qui consiste à laisser sur chaque coupe après son exploitation:

Par hectare: 100 baliveaux de l'âge de . . 30 ans.

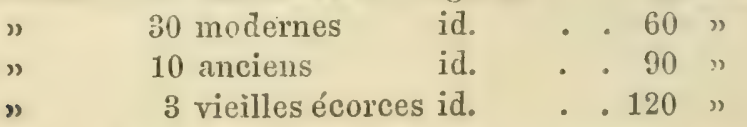

I Ainsi, dans les forêts de l'Êtat et des communes, le plan de balivage consiste à réserver 50 baliveaux de l'âge par hectare et à unabittre les modernes et les anciens que lorsqu'ils sont dépérissants ou hors d'état de prospérer jusqu'à une nouvelle révolution (urdonnance du 1 er aoút 18:7, art. 70 et 131$)$. 
Le matériel d'exploitation, en ce qui concerne les réserves, sera, dans chaque coupe, composé de:

400 baliveaux.

120 modernes.

40 anciens.

12 vieilles écorces.

Ce matériel sera pour toute la forèt de:

12000 baliveaux âgés de . . . 30 à 60 ans. 3600 modernes id. . . . . 60 à 90 " 1200 anciens id. . . . . 90 à 120 " 360 vieilles écorces âgées de. . . 120 à 135 "

17160

Le caractere essentiel du matériel d'exploitation des futaies sur taillis est de comporter une succescession d'âgres gradués sur chaque coupe et non plus sur l'ensemble de la for'êt. Cette disposition permet de le íaire varier à chaque année de l'exploitation, selon l'état de chaque coupe et selon les convenances du propriétaire. Le traitement des forèts en taillis sous futaie, qui a peut-être été trop décrié, convient ainsi à des situations de fortune très-diverses, aux palticuliers comne aux communes.

Compte d'aménagement. - Par cela même rue le plan de balivage est susceptible de nombreuses variations, il est nécessaire d'en tenir un compte exact.

Il furt, en effet, que le proprietaire puisse toujours se sendre comple de l'étal de sat forèt. Si sa fortune 
comporte une grestion importante, il faut que ses contróleurs puissent, à tout moment, vérifier les opérations des réģisseurs. Il est arrivé trop. souvent, même dans les grandes administrations, que les régisseurs, désirant plaire au propriétaire, ont abusé des ressources de la forêt et ne lui ont laissé, après une gestion séduisante par ses revenus, qu'un immeuble sans valeur et sans avenir.

Une autre raison doit engager aussi certains propriétaires à tenir cette sorte de comptabilité. Il y a des liquidations de communautés qu'il faut saroir faciliter sous peine de compromettre la paix des familles; et dans toutes les situations usufruitières, les arbres de futaie ne sont acquis à l'usufruitier que lorsqu'on peut, au moyen de documents certains, préciser exactement le plan d'exploitation, l'aménagement du propriétaire ${ }^{1}$.

Le cadre de comptabilité que nous avons donné pour l'aménagement des taillis s'adapte parfiritement au compte des futaies sur taillis. Il faut noter cependant une différence, non daus la forme, mais dans la nature de ce compte. Quand il s'agit de la comptabilité des coupes par contenance, on est certain qu'une coupe exploitée se représentera à nouveau, c'est-à-dire au compte de la rérolution suivarite, avec la mème contenance. Il suffit donc de

1 Ccde civil, art. 591. 
noter, dans les comptes, que celle-ci a été explu,tée. Il en est autrement pour les arbres de futaie; chaque coupe en présente un nombre rariable, dans lequel on choisit, chaque année, ceux que l'on reut abandunizer (exploiter) et ceux que lon reut réserver (conscrver). Il fiut done terir note à la fois de cet aliandon et de cette iéseire'. Sans cela, les contròleurs ne sauraient exactement vérifier les coupes; le proprietaire ne pourrait se rendre compte de l'état de sa forèt.

Dans l'exemple précédent, les 120 hectaress'exploitent it 30 ans, à raison de 4 hectares par an; il n'y a pas de réserre assise sur le terrain. Le compte de la fulaie sera tenu de la facon suivante:

1 Il u'est pas inutile de faire observer qu'il ne s'agit pas ici d'une mesure de fréroyance analogue aux quarts de réserve. Rien n'empécherait de faire une résel fe par roie de prélèrement sur le plan de balivage: cula pent aroir une utilité et dispenser d'un quart de réserre. Dans les exploitatins de folêt au la préroyance du propriétaire a été assurée par une partie speriate de la forêt ou par des compons dont on retarde la coupe, cute partie spéciale ut cer co:ty.ms sont alors garnis de leurs arbres de firtuie, et il est peu utile de fatio un prélivement syécial sur le plan de balirage. 


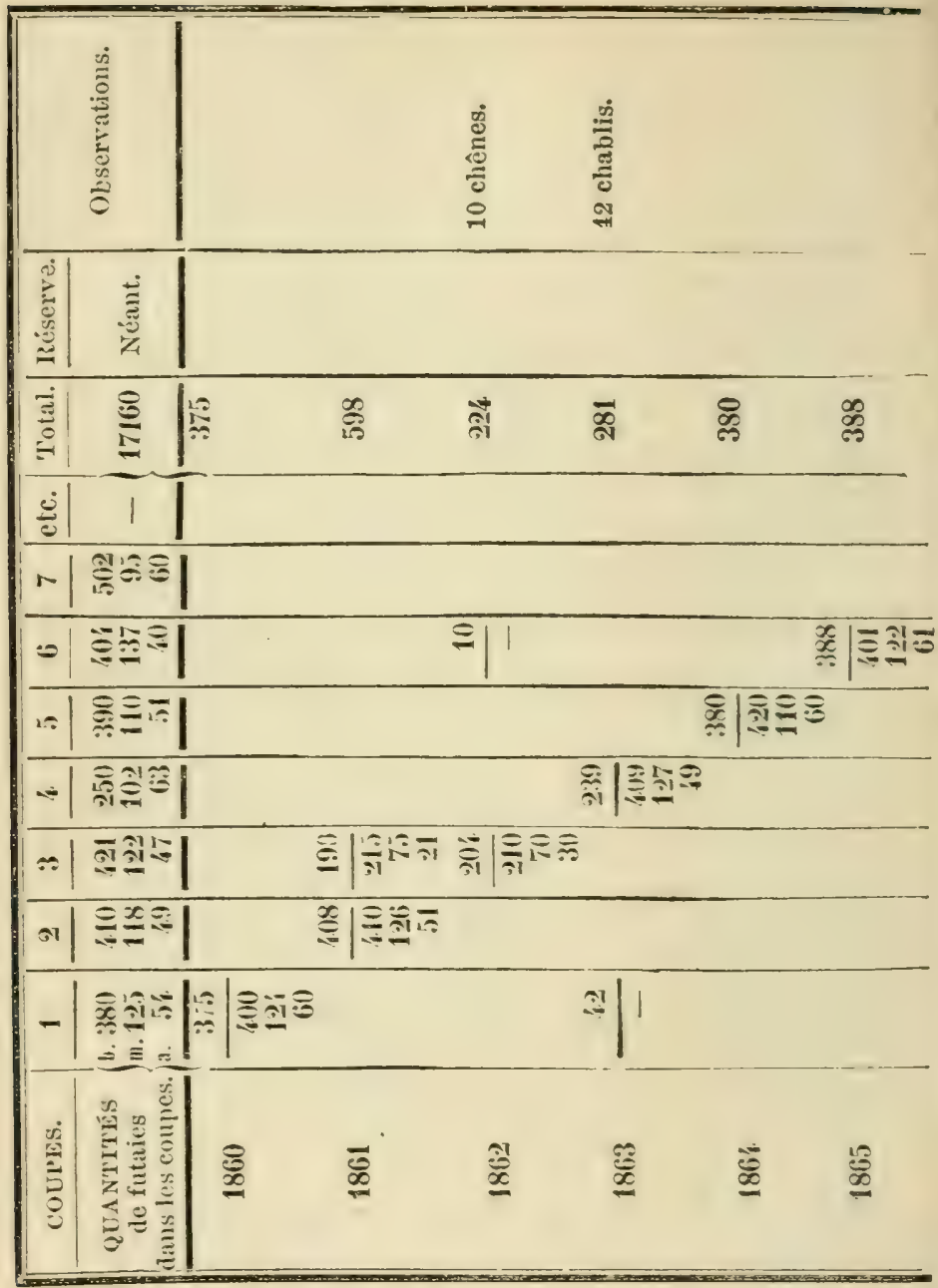

On a compté tous les arbres de futaie qui se trouvent dans chaque coupe, et on les a inscrits en tête du compte en les attribuant aux trois catégories principales d'âges : $b a$ liveaux, modernes, anciens. Vis-ì-vis de chaque année on 
trace, dans la colomne correspondante à la coupe, un trait lurizuntal au-dessus duquel on inscrit liabandon et audessuns, la réserre. L'inspection du compte montre immédiatement qu'en 1860 le propriétaire a exploité la coupe n० 1: en 1861, la coupe $n^{\circ} 2$ et la moitié du no 3; en i862, il est rentré dans l'aménagement en n'exploitant que la seconle moitié de la coupe $n^{0} 3$, mais il a rendu 10 chênes I an anticipation dans la coupe $n^{\circ}$ b; en 1803 , on a rendu 4: chathlis dans le no 1 et expluité la culupe no 4 , etc., etc.

La réserve est indiquée dans le cumpte par trois chiffies qui doment le nombre des baliveaux, des modernes et des anciens; mais l'abandon est simplement constaté par le elifire, en blue, des arbres mis en rente. Il est inutile et il scrait sans intérêt de repruduire les catéguries d"arbres de lahandon. Ainsi, la coupe $n^{0} 1$ présentait 559 arbres de furaie de toutés catéguries: on en a conservé 184 et par conséquent abandonné à l'expluitation 375. Les 400 baliveaux ont été choisis dans le taillis. A la rigueur, la balance devrait être exacte, e'est-à-dire que le chiffire de l'abandon, 375, derrait être égal ì la différence entre le nombre tutal des arhes existant sur la coupe et le nombre des arhres (autres que les baliveaux) réservés. On conçoit que lixactitude de cette balance ne saurait itre mathématique; il y a des erreurs de comptage, des accidents, des déchets, dont il fant savoir tenir compte dans les exploitations de furits comme dans tuutes les industries. Les régisseurs inscrivent le résultat de leurs opérations: c'est aux rérificateurs ì savoir apprécier.

Co comple des arbures de futair est le complément du compite de lamémagement des coupes de taillis. On pourait fort hien indiquer la contenance des compes dans les enlonnes du compte, mais il est prefirable dem tenir desux, l'un pour lat futaie. latutre 
pour les coupes de taillis. Une comptabilité r'a de mérite que par la simplicité de son organisation, et plus les intérêts à administrer deviennent considérables, plus cette simplicitéa d'utilité pratique. Nous ne craignons pas d'anticiper sur les chapitres sujvants en faisant remarquer que:

10 La forme des comptes d'aménagement est conçue de manière à s'adapter à tous les moiles d'aménagement, à toutes les natures de coupes.

$2^{0}$ La seule règle à observer est d'ouvrir un compte séparé pour chaque nature des compes qui s'exécutent dans une forèt suivant un plan déterminé. 


\section{TROISIÈME PARTIE.}

\section{Anénagement des forèts à gros capital d'exploitation.}

\section{FUTATES,}

\section{CH APITRE PREMIER.}

FUTAIFS PIAINES.

Au point de rue économique, la futaie est un urme d'exploitation des terres à jois qui nécessite un gros matériel; dans le sens cultural, ce mème not signifie un régime dans lequel les arbres se reproduisent par les semences. Le traitement en futair peut se faire de deux manieres: en futaie pleine, morle dans lequel on cultive sur des contenances séparécs les peuplements de chaque catẻgorie d'àges, et en futaie jardinée, méthode particulière, qui seri l'objet du chapitre II.

Je suivrai, pour les futaies, le mème ordre que pour les taillis, c'est-a-dire qu'en adoptant pour exemple un terrain de 120 hectares, it exploiter it ('ent vingt ans, j’indiquerai d'abord les procédés yu'on emploie pour aménager une forèt dont le ca- 
pital de un ì cent vingt ans est constitué et se trouve disposé d'une manière assez réğulière sur le terrair pour pouvoir pigndre le nom de futcic reguliere.

Je passerai sous silence les procédés qu'on em. ploie pour déterminer l'ìge de l'exploitahilité la plu: avantageuse pour lé propriétaire. Cies procédés ne sont pas du domaine d'un livre élémentaire. Généra lement les propriétaires se contentent de compter le: années au moyen des couches concentriques sur de bois abattus et de roir à quel àge les arlures acquie. rent, en moyenne, les dimensions qu'ils ont intérè à produire.

\section{$\$ 1 \mathrm{cr}$.}

Futaies dont le matériel est constitué.

A.

Aménagement des futaies dont le matériel est constitu et régulièrement disposé.

Deux natures de produits de la futaie. - Aran d'indiquer les procédés d'aménagement qui permettent de constater à première rue si le capita d'exploitation est toujours intact, et de tracer l'ordre dans lequel se feront les coupes, il n'est pas intitile de rappeler que, dans une exploitation en futaie, le coupes sont de deux natures, l'une et lautre fondéc sur la marche naturelle di la régétation des hois. 
Lit premiere condition de l'exploitation en futaie -t dr rógénérer maturellement la forèt, c'est-à-dire ar les semences des arbres. On fiit done, dans les ois exploilables, des coupes d'ensemencement dans styulles les arbres porte-graines sont assez serrés our donner la fraicheur nécessaire à la levée des raines; elles sont suivies de coupes secondaires, ans lesquelles les arbres sont assez espacés pour iiser les jeunes plants se développer ; puis rient la oupe définitive pour les débarrasser entièrement u courert des rieux arbres. L'ensemble de ces difirentes coupes, qui ne sont que les phases d'une rème opération, s'appelle coupes de régénération. e sont ces exploitations qui fournissent les produits incipanx de la forèt, puisqu'elles sont les plus mportantes et qu'elles portent sur des bois parrenus l'ìge adopté pour l'exploiiabilité i.

Toili la première nature de produits que l'on peut etirer d'une exploitation de forèt en futaie pleine; nais là ne se borne point sa production. Si l'on conidire des jeunes bois en croissance, et si on les suit ussqu'au moment où ils sont exploitables, on voit bien.

1 Cimme, en réalité, on n'aménage que des forêts plus ou moins irréuliires, on don:ae aussi le nom de produits principanx aux produits des mutes dextraction d'arbres fates dans les futaies irrigulieres. Les pro. uits prinipuo des futaies sont done, d'une maniere génirale, ceux qui utrent daus le calcul de la possibilité dés conpes par volume. Tons dénirons plus loin la possibilité. 
vite que beaucoup deriennent dominés er finissent par périr étouffés: un massif déloute, par exemple, par dix mille tiges à l'hectare, et finit, à cent ving ans, par n'en plus offrir que trois cents. La végétation des massifs suit à cet égard une loi qui nous est en. core inconnue et dont la connaissance serait fort utilc pour lia conduite des opèrations forestières (r. p. 2j) La physiologie végétale fournit heureusement aus forestiers une règle de conduite qui supplée, dans une certaine mesure, à cette loi expérimentale.

La production ligneuse est proportionnelle au développement foliacé, et le ce principe plysiologique théoriquement prouvé, il fiut conclure que si on i soin d'enlever, de temps en temps, les tiges dominées en laissant le massif complet ${ }^{1}$, on arrivera à ne né gliger aucun élément de la production d'un bois car on enlève des tiges qui, sans cela, auraient péri et on n'amoindrit en aucune façon la production e le volume de l'hectare exploitable. C'est sur ceft considération qu'est fondée la pratique des éclaircies qui constituent des produits intermédiaires don l'importance va jusqu’à 15 et 25 p. 100 des prodıi' principaux.

L'aménagement des futaies doit done avoir pon

1 L'état de massif complet est cunstitud quand les branches et les cime rles arbres se touchent sans être agitées par le rent (MLM. Lercntz et P: rade, Cour's de culture des bois, 110406 ). 
but d'assurer la marche de cette double nature de coupes, et nous l'envisagerons d'abord au point de rue des coupes principales qui, dans une futaie réguière, se bornent aux coupes de régénération.

Affectation. - Danś l'exploitation en taillis à fingt ans, nous avons divisé la surface en vingt lots, lont chacun était affecté à la coupe annuelle: nous ouvions le faire, parce que, dans les taillis, il suffit l'm an pour assurer la régénération, et parce que es rejets peurent, dès la première année, ètre abanlonnés à eux-mèmes, sans avoir besoin d'aucun bri. Mais, dans les futaies, les choses ne se passent oas ainsi: les semences ne se produisent pas imméliatement, le jeune plant, une fois levé, a besoin 'une certaine protection, d'un abri plus ou moins rrolongé selon les essences, et ce n'est sourent qu'au out d'un temps assez long qu'il peut ètre abandomné lui-mème et que la coupe doit ètre considérée comme complétement régénérée. Si donc nous divi:ions nos 120 hectares en cent ringt affectations d'un nectare, correspondant chacune à lia coupe annuelle, nous ne donnerions satisfaction à aucune de ces exigences culturales.

Il ent done rationnel de diviser la forèt, non pas 'n cent vingt coupes, mais en un certain nombre de norecaux affectés à fournir la production pour la vériode de temps peindant luquelle, inon-seulement 
le terain peut se repeupler naturellement, mai pendunt laquelle, en outre, les semis peurent, un fois produits, se passer de tout abri, de toute pio. tection.

Cette durée, qui rarie arec les climats, les sols ol les essences, s'appelle période de régénération, on simplement période. On conçoit que sa détermination est une affaire d'expérience et que nulle science autre que l'obser'ration ne peut en donner la mesure. Le propriétaire qui reut aider la nature par des travaux et par cles semis artificiels dont il ne craint pas la dépense domnera à la période la durée la plus courte possible; celui qui s'en rapporte uniquement aux agents naturels sera, au contraire, porté à l'allonger. En cela, comme en toute affaire, c'est une sage entente de ses intérèts qui doit guider le propriétaire. Dans la balance qui sera faite entre les arantages et les inconrénients, on ne saurait oublier que les arbres laissés sur pied pour abriter le sol et protéger les semis compensent généralement, par leur accroissement, le retard que met sourent la nature à assurer la rẻgénẻration d'une coupe.

Supposons, pour fixer les idées, que l'inventaire du matériel actuel, l'amalyse de la forèt, le parcellaire enfin, pour l'appeler par sun nom forestier, ait donné: 
A. 6 hect. chêne clair avec semis . . . 130 ans.

B. 5 " hêtre presque pur avec semis . . 110 "

C. 9 " hêtre, frêne et chêne . . . . 105 "

D. 10 "haut perchis sapin et hêtre. . . 90 "

L. 15 " haut perehis un peu clair, sapin . 80 "

F. 14 " 50 ares perchis complet, sapin - 70 "

G. 2 " 50 " id. épicéa . 50 "

H. 27 " 50 " sapin et hêtre . . . . 40 "

I. 22 " 30 " gaulis de hêtre . . . 30 "

J. 8 " 50 " semis de hêtre et sapin . 5 "

120 hectares.

Dans celte forèt, le matériel d'exploitation est onstitué, car', dans les futaies, il ne faut pas s'arrèel à certains écarts dans les âges et dans les peulements. Cornme les parcelles d'ages roisins se nivent de proche en proche et comme les pertplenents correspondent, à peu près, à leurs conditions e régétation, la constitution du matériel permet de onsidérer la forèt comme r'égulière. Si la durée de a période de régénération a été fixée à vingt ans, a contenance des affectations sera de 20 hectares $t$ leur nombre de six.

Dans les taillis, on a divisé par des laies les vingt oupes affectées à la production de chaque amnée; lans les futaies, on groupera de mème les parcelles u parties de parcelles qui doivent former chaque flectation; on marquera sur le terrain, par des laies éparatives, chacun des six morecaux affectés à la moduction de chaque vingtaine d'années, et on les iscrat Dar les Jornes. 
Le principe de cette division en affectations égales est fondé sur la supposition qu'elles produiront autant l'une que l'autre. Cela peut ne pas arriver et n'urrive même souvent pas dans les forèts de montagnes, où les pentes produisent généralement plus de bois que les plateaux, et où les expositions ont une grande influence sur la régétation. Si de pareilles inégalités se présentaient, on se contenterait d'ajouter quelques hectares aux affectations les moins bonnes, de manière à les rendre équiproductives autant que possible; mais il $\mathrm{y}$ a là une aflaire d'appréciation et de pratique qui ne peut se mesurer' ni par des coefficients ni par des méthodes exactes.

\section{Plan général d'exploitation. - Dans les taillis,} le plan général des exploitations se compose des vingt coupes affectées à chacune des années de la rérolution; dans les futaies, il se compose seulement des six morceaux affectés à chacune des six périodes de la rérolution: chacune de ces six affeclutions peut se composer de plusieurs parcelles diflërant par leur àge, leurs essences ou le traitement à appliquer. Il convient, dès lors, de conserver les lignes des divisions qui ont servi à faire l'analyse de la forèt et de les marquer, soit par des hornes, soit par des bouts de fossés. On comprend tris-bien que si la parcelle A, par exemple, est peuplée de chènes dont le semis soit réussi, il faudra plus tòt y porter les 
coupes que dans la parcelle $B$, dont les hètres supportent bien le couvert. Il sera, par conséquent, fort important de savoir le volume des bois qui auront èté enlerés sur chaque parcelle et de connaître, à un moment donné, ce qui reste à exploiter. On comprendra mieux l'utilité qu'il y a à conserver sur le terrain la trace de ces parcelles; quand j'aurai indiqué la manière dont se tient le compte d'aménagement; mais dès à présent on conçoit l'utilité de conscrver sur le terrain le travail d'analyse qui a été liait; les régisseurs se perdraient dans l'exploitation de la forèt aussi facilement que dans une ferme dont les soles et les saisons ne serajent pas abornées. Lors de leur création, les parcelles ont été désignées par des lettres ou des numéros; une fois leur classement opéré dans chacune des affectations, il est for: utile de les désigner par leur numéro d'affectation auquel on ajoute les lettres $a, b, c \ldots$ selon les différences de peuplement qui ont servi à les créer; on a ainsi : I $a$, I $b$, I $c$; II $a$, II $b$, II $c$; III $a, \operatorname{III} b \ldots$ Ce mode de notation indique immédiatement a quelle affectation apparticnt la parcelle et, par consé(uent, à cuuel moment la coupe s'en fera; il empèche les fautes grossières d'exploitation qui peurent arriver' aux changements de régisscur's; il écrit enfin sur le terrain le plan général d'exploitation, c'est-i-clire' le carlre que l'on doit toujours avoir devant les youx 
et dans la mémoire quand on veut exploiter convenablement une futaie.

Ce cadre général, ne contenant que six affectations, sera donc très-simple:

\section{Pian gènéral d'exploitation.}

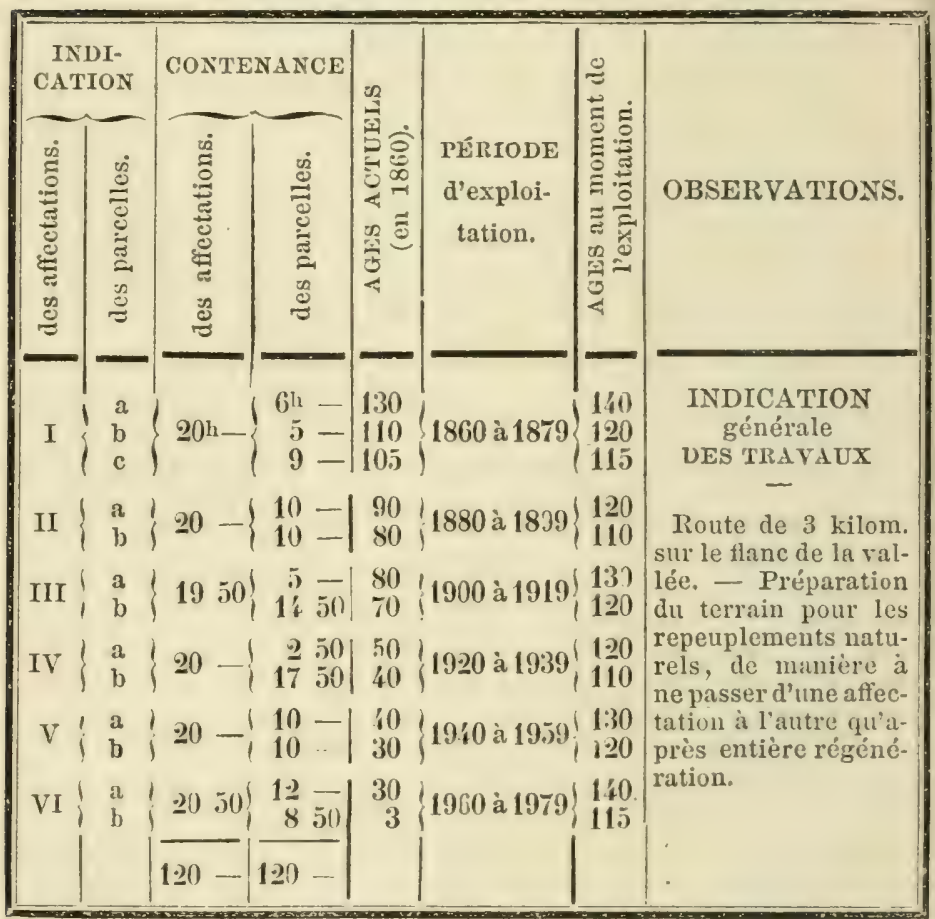

Il semble, au premier abord, (fue ce plan généıal ne s'adresse qu'aux coupes principales, puisqu'il ne détermine que les contenances qui seront régénérées dans telle période d’années. Il est évident qu’il 
s'arlresse aussi aux éclaircies, car, au vu de ce tableau, l'on saura toujours qu'on ne doit pas faire d'éclaircie dans l'affectation no I destinée aux coupes de régénération; que dans l'aflectation $n^{0} \mathrm{VI}$, qui ne contient que des jeunes semis, l'éclaircie se bornera le plus sourent à un simple nettoiement; on samra toujours, enfin, que les réritables éclaircies se feront, en gènéral, dans les affectations II, III, IV et $\mathrm{V}$.

Plan spécial d'exploitation. - Ainsi, ce plan général d'exploitation est complet et comporte, pour de larges coupures faites dans la durée totale de la révolution, toutes les coupes qui peurent ètre opéries, la détermination mème du matériel d'exploitation. Mais il n'est pas suffisant el l'opération de l'aménagernent comporte un second lut, qui est de régler la quotité, lia nature, l'ordre et la marche des coupes qui, tout en laissant intact ce matériel, constituent la production annuelle ( . p. 33); c'est le but du plan spécial d'exploitution. Un propriétaire soucieux d'une bonne administration ne peut se contenter de saroir qu'il n'entamera pas son capital en filisant ses exploitations dans l'aflectation en tour de riginérution (ou plus simplement dans l'affectation entour). Cetterérification, tous les vingt ans, ne peut lui suffire; il lui faut d'ailleurs prendre les mesures necessaires pour égaliser, chaque année, les produits 
à réaliser dans le cours de cette période de vingt ans. Dè là, nécessité de tracer, à l'avance, la marche des exploitations à faire dans cette période, de manière à donner au propriétaire la conviction qu'il ne dépassera pas le chiffre de la production annuelle possible, c'est-ád-dire de la possibitité, en langage forestier.'

Il y aura done un plan spécial d'exploitation pour chaque période, et ce plan spécial comprendra: 10 les coupes de régénération à faire dans l'affectation en tour, 2 o les coupes d'éclaircie ä faire dans les autres uffectations. Comme il est impossible de préroir l'arenir et l'état des peuplements au dela d'une certaine limite de temps, on ne dresse ce plan spécial que pour la première période, en laissant à la fin de chaque période, vers $1879,1899,1919$, etc... le soin de dresser, pour la période dans laquelle on entrera, ce plan spécial dont actuellement le propriétaire n'aurait nul besoin.

1. Produits principaux. - Non-seulement ce plan spécial doit comprendre les coupes de récénération et les coupes d'éclaircie, mais il doit encore donner pleine et entière salisfaction aux exigences culturales que ces coupes comporient. Ainsi, en ce qui concerne les coupes de régénération, principale

1 La possibilité est la quotité des produits que l'un pent retirer anmuellement et d'nne manière const:mte d'un immeuble exploité dans des con ditions données, sans le détéıiorer 's lon le langage des économistes), en cunserfant sa substance (selon lexpression des juristes). 
production de la forèt, la contenance ne peut plus servir de base à leur assiette: si elle était admise, on pourrait avoir, une année, beaucoup d'arlores à exploiter et fort peu l'année suivante, car les semis naturels sont loin de se présenter et de se développer avec régularité. Les coupes se font alors par volume: on se contente d'estimer et de cuber le volume qui se trouve dans l'affectation en tour et de diviser cerolume par le nombre des années de la période.

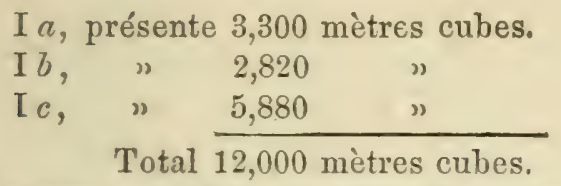

La coupe sera $1 / 20 \mathrm{e}$, soit 600 mètres cubes.

Il est érident qu'en coupant tous les ans 600 mètres culbes, on sera sûr de ne pas dépasser la possibilité, car les arbres qui peuplent la première affectation présentent ce matériel actuellement, et, comme ils resteront tous en moyenne sur pied pendant la moitié de la période, c'est-à-dire pendant dix ans, il serait fort juste d'ajouter au matériel actuel, 12,000 mitres cubes, l'accroissement pendant dix ans. Je me hàte de dire que cet accroissement est toujour's assez difficile à déterminer et qu'il est beaucoup plus prudent de se contenter, pour la fixation de la coupe anmuelle, du vingtième du volume constité au moment oì l'on fait l'aménagement. 
L'accroissement formera une réserve pour parer à la diminution de volume qui peut venir des erreurs d'estimation et de cubage ${ }^{1}$; il ne sera jamais perdu, car rien n'empèche de vérifier, de tenons en temps, le volume restant à exploiter, et de fixer à noureau le chiffre de la coupe, en le divisant par le nombre des années de la période qui restent à courir. Cetle opėration fort simple, qui s'appelle révision de la possibilité, peut se faire, par exemple, à chaque décennie.

Quant à l'ordre des coupes, c'est à la nature et au sylviculteur qu'est réservé le soin de les indiquer; rien n'est capricieux comme la manière dont se produisent les semis naturels, et tout ce que l'on peut faire, c'est d'indiquer l'ordre dans lerquel on commencera les coupes; cette indication se fuil simplement par les indices $a, b, c$, dans le plan d'exploitation.

20 Produits intermédiaires. - Ainsi les produits principaux s'exploitent par volume; c'est mème sous ce titre qu'on les range dans le plan spécial l'exploitation, par opposition aux coupes d'éclaircie Iui se font par contenance. Ces dernières, qui sont

1 Les chablis et les bois morts qui se présentent dans la première affectation et en général dans les parcelles qui fournissent la possibilité des coupes par volume sont précomptés sur la coupe anmuelle. Ceux qui surviennent dans les autres parties de la forêt sont expluités sans précompt:lge, puisqu'ils n'entrent pas dans le calcul de la possibilité. 
sourent appelées, d'une manière générale, coupes d'amétioration, ont un caractère qui diffère arec. l'iige des bois et par conséquent arec le classement des massifs dans les affectations. Dans les jeunes bois, le nombre des brins qui périssent itouffés est naturellement plus grand que dans les Joois plus âges ; l'éclaircie doit done y ètre faite à des intervilles plus rapprochés. Dans les bois de quatrevingts à cent ans, qui sont classés en $\mathrm{Il}^{\mathrm{e}}$ affectation, il est sourent utile d'éclaircir un peu fortement, afin de permettre aux arbres qui seront plus tard des somencier's de prendre de la tète et de devenir. aptes à la production des graines: l'éclaircie prend alors un caractère nouveau qui la fait distingucr des autres sous le nom de dernière éclaircie ou priparatoire a l'ensemencement.

Le propriétaire qui reut consacrer tous ses soins à la gestion de sa forèt cher'hera done à répéter assez sourent ces opérations délicates et à leur donner une rotation conforme it leurs diflérentes exigences; mais dans une gestion importante, comme celle de l'aluninistration des forèts, il suffit que le règlement des iclaircies s'alapte aux principales exigences des peuplements et aux traits généraux de leur pratique culturale.

\section{Deux systèmes sont usités :}

10 Dans le premier, chacune des quatre affecta- 
tions qui doivent être éclaircies forme une suite de coupes qui doivent parcourir l'affectation une ou deux fois pendant la durée de la période. Ainsi, dans les affectations II et III, on éclaircira, tous les ans, un vingtième de la contenance ou 1 hectare par an. Dans les affectations IV et $\mathrm{V}$, on éclaircira, tous les ans, un dixième de la contenance ou 2 hectares par an, en passant deux fois dans la période sur toute la surface. On peut même apporter dans cette pratique une régularité analogue à celle des taillis, en divisant chaque affectation par dixièmes de contenance et en faisant les coupes tous les deux ans dans les affectations II et III, et tous les ans dans les affectations IV et $\mathrm{V}$.

Ce système a l'avantage incontestable d'imprimer aux coupes d'éclaircie une marche très-régulière et de contribuer à assurer le rapport soutenu en fournissant chaque année des éclaircies ayant le mème caractère, c'est-à-dire faites dans chaque affectation dans les mêmes natures de peuplement. La seule critique qu'on peut lui adresser, c'est de fournir, dans les forêts de faible étendue, des coupes trop petites qui donnent peu de produits, sont difficiles à vendre et divisent trop la surveillance.

Le plan spécial d'exploitation de la première pé-. riode sera, dans ce système, dressé de la façon suivante: 


\section{Plan spécial d'exploitation pour la première période (1860-1879).}

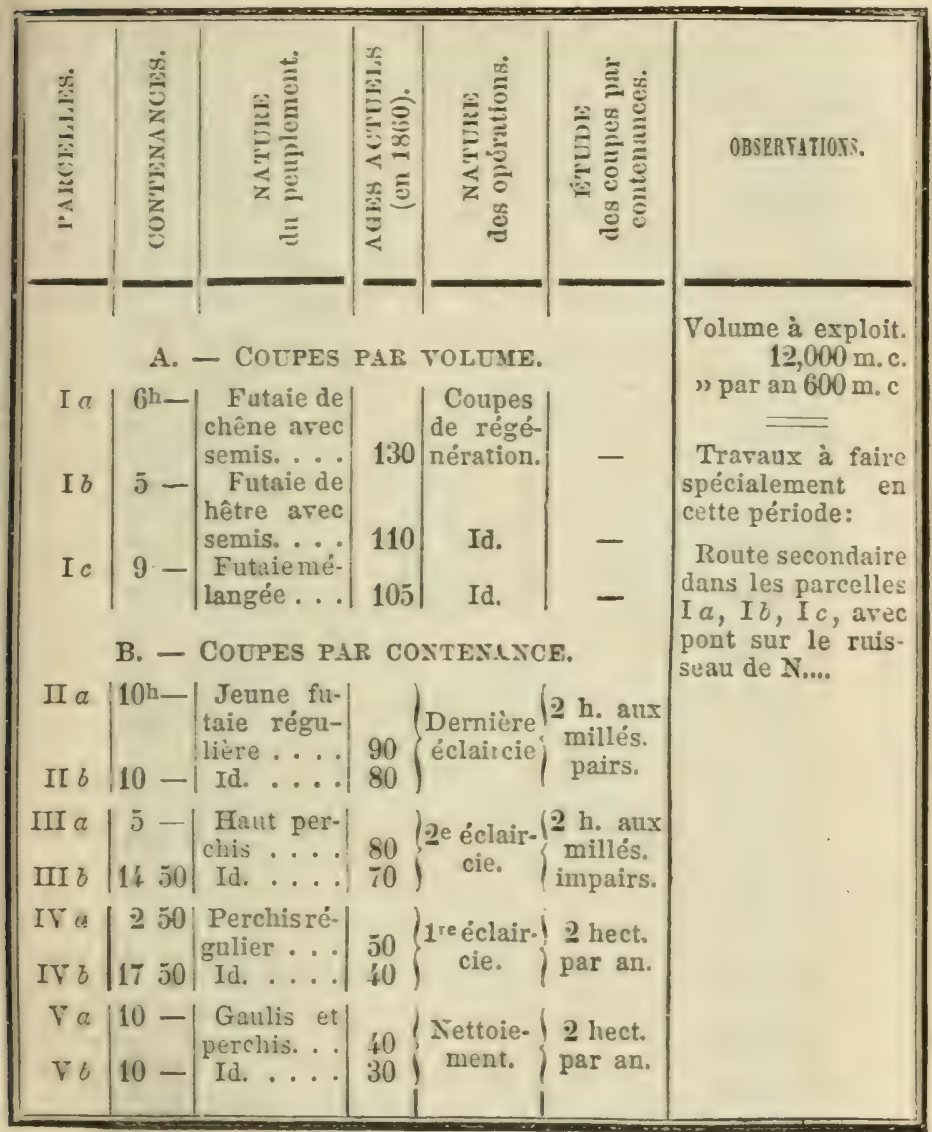


Le plan topographiqne de la forêt sera le suivant :

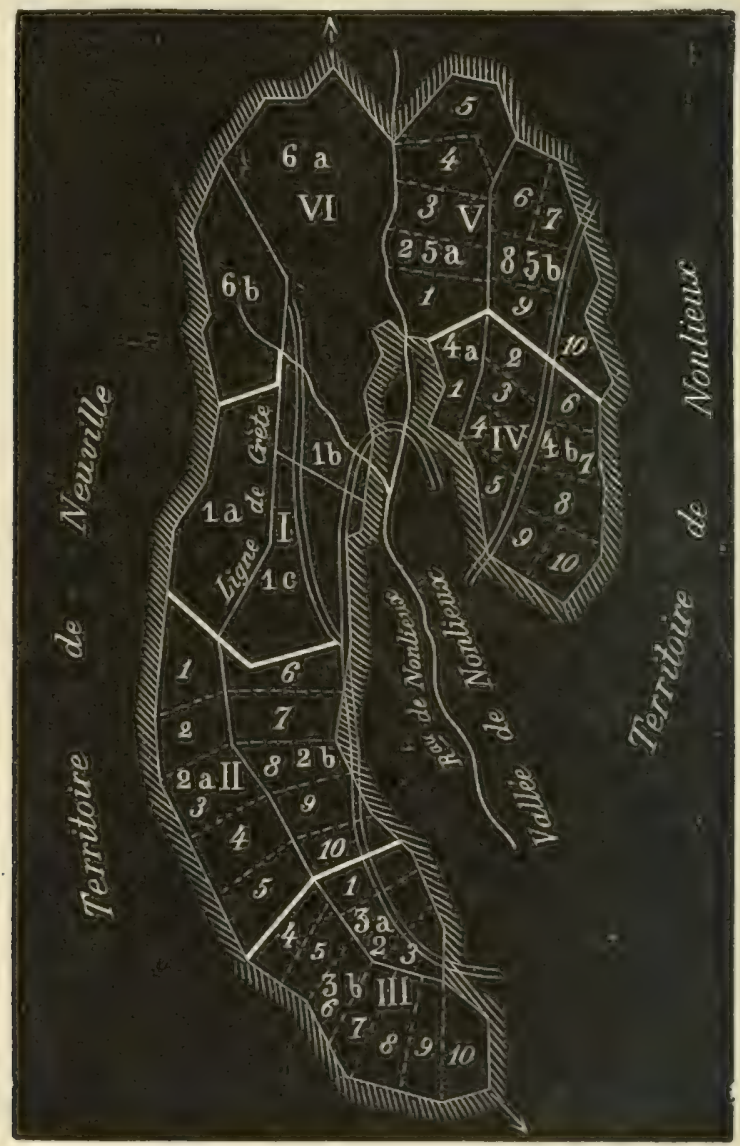

Fig. 7. - Aménagement en futaie.

$2^{\circ}$ Dans l'autre système, on fait masse des quatre affectations à éclaircir, et lẹur étendue de 80 hec- 
fares forme une suite de coupes pour le temps qui a été choisi pour la rotation des éclaircies. Il scra toujours a vantageux, comme dans le premier systèue, de prendre pour cette rotation la durée même de la période; ainsi les éclaircies dureront ringt ans, à raison de 4 hectares par an, et on restera pendint cinq ans dans chaque affectation. Le caractère de léclaircie scra indiqué par l'affectation dans laquelle elle se fera, et la rotation qui est adoptée pour les parcelles est bien de nature à retracer ce caractère à l'esprit des employés chargés de diriger la coupe. Si celle-ci se fait, par exemple, dans la parcelle IVı, le plus modesie garde saura toujours qu'il s'agrit d'une coupe d'éclaircie dans une qua'rième affectition, bien qu’il n'ait à sa disposition aucun plan, et que l'aménagement de la forèt lui soit inconnu.

Al la rotation suirante, c'est-à-dire en 1880 , la II aflectation viendra en tour de régénération et sera romplacée, dans la suite de coupes des éclaircies, par la VI affectation, de manière à maintenir au complet le chiffre de 80 hectares; on repassera ainsi sur les coupes tous les quinze ans, si l'ori reut continuer à faire les éclaircies dans l'ordre des affectations, - ce qui est une moyemne entre linterralle conrenable pour les jeunes bois et les bois plus àgés, - ou bien tous les vingt ans, si l'on a soin de commencer les éclaircies en 1880 par l'affec- 
tation qui entre à nouveau dans cette suite de coupes, pour remplacer celle qui vient en tour de régénération. On peut donc, à chaque période, repasser sur les coupes à un intervalle convenable pour les cxigences culturales, et on introduit la simplicité et la régularité des taillis dans les aménagements de futaie, en divisant chaque affectation par cinquième de sa contenance.

Le seul inconvénient de ce système est de ne pas fournir un rapport bien soutenu, car les éclaircies dans II et III sont bien plus productives que celles qui se font dans IV et $\mathrm{V}$ et qui se bornent le plus souvent à de simples nettoiements ; mais sa simplicité l'a fait souvent adopter dans les forèts importantes appartenant à l'État ou aux communes, et où l'inégalité de la production est le plus souvent compensée par la combinaison de ces coupes avec celles d'autres séries d'exploitation.

En resumé, l'on peut dire que le choix entre les deux systèmes dépend de la contenance de la forêt à aménager : le premier est préférable quand il n'a pas pour résultat de livrer à l'exploitation des coupes de trop faible importance.

Voici le plan spécial d'exploitation dressé pour le deuxième système de règlement d'éclaircies : 


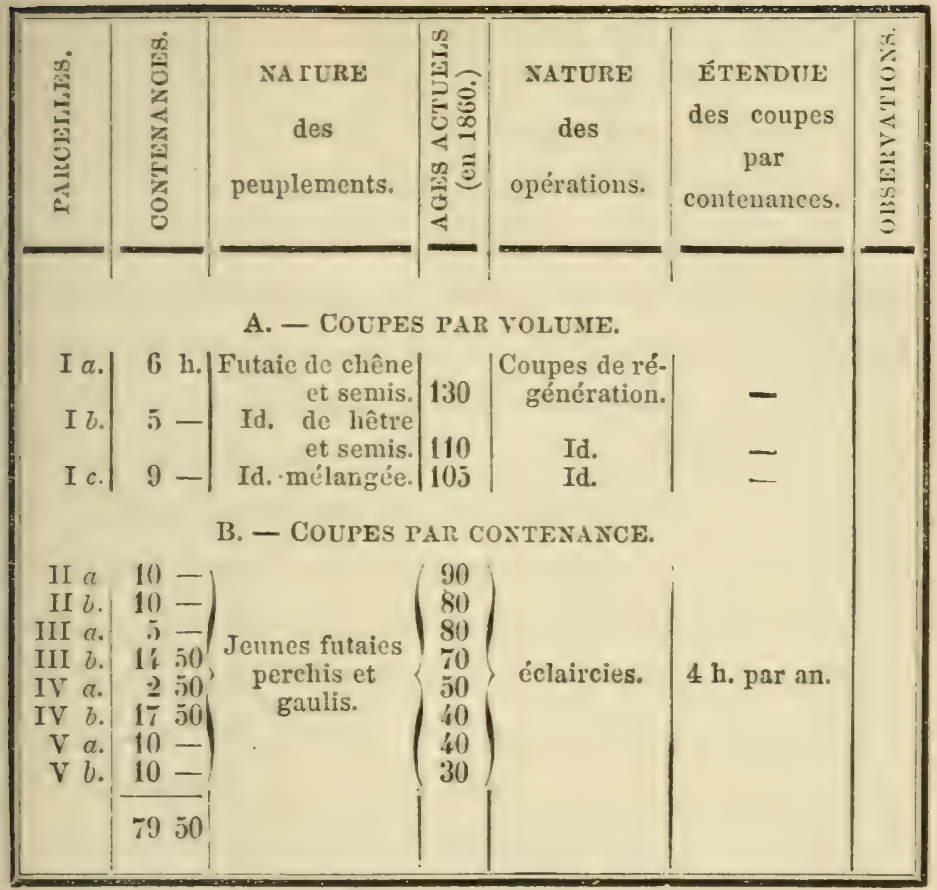

\section{Revenons à notre forêt :}

Voilit done trois natures de divisions établies dans la forèt : les affectations; les parcelles; les coupes d'écluircie. Les premières sont marquees sur le terrain d'une manière fixe et permanente : ce sont les grandes divisions de la forèt; les deux autres ne sont que des sous-divisions des affectations, et l'on peut se demander s'il est nécessaire the les asseoir ct de les marquer sur le terrain.

Il est diflicile de poser à ret égard une riogle ab- 
solue. En ce qui concerne la première affectation, nul doute à avoir ; les lignes des parcelles derroni toujours être marquées sur le terrain, parce qu'il sera toujours utile de savoir, à un moment quelconque, le volume de bois qu'elles contiennent, et qu'il leur sera ouvert, à cet égard, dans la comptabilité de l'aménagement, un compte spécial à chacune. - En ce qui concerne les autres affectations, il peut se présenter des forèts assez régulières, assez homogénes pour permettre de supprimer les parcelles qui ont servi à en faire l'analyse et pour asseoir sur le terrain les lignes des coupes d'éclaircies. Les lignes des parcelles ne seront ainsi conservées que sur les plans et y figureront à titre de renseignement; les lignes des coupes seront marquées sur le terrain par de petites bornes ou des bouts de fossés. Nais le cas sera rare, car on n'aménage, en réalité, que des forèts irrégulières; or il faut bien se rendre compte du rôle des parcelles : créées d'abnrd pour faire l'analyse de la forêt et permetlre d'en apprécier les ressources, elles ont, en outre, pour but définitif de séparer les parties dont la situation et les circonstances de la végétation imprimeront, d'une manière permanente, un cachet spécial au traitement des peuplements. Ainsi leur double caractère est d'ètre des organes d'analyse et des divisions culturales. Une fois le plan général 
'exploitation établi, on doit supprimer les parcelles ui n'ont été que des organes d'analyse, et ne conerver que celles qui, établies en vue de particularités ermanentes, conserveront leur raison d'ètre. On levra done, en général, marquer sur le teriain es lignes des parcelles. Quant aux coupes d'éclairies, on les tracera sur le plan de la forèt, en leur lonnant la forme la plus convenable pour la vidange, clon le relief du terrain; on évite ainsi les pertes le temps et les tàtonnements qui peurent aroir lieu chaque année de leur asșiette. Dans la protique, in modifie mème un peu la règle absolue de division ar contenances égales, que nous arons donnée pour 'exposé des deux systémes de règlement d'éclaircies; n utilise les parcelles en les groupant ou en les cindant, de manière à former des annuités de coupes l'éclaircies dans les limites des purcelles, arec des carts tolérables dans les contenances annuelles; et 'on porte au tableau du plan spécial ces contenances unnuelles en regard des années où elle's derront 'exploiter. On satisfait ainsi, d'une manière comolète, it une des conditions les plus essentielles d'un on aménagement, qui est de ne jamais entraver et le faroriser toujours l'application des règles de la mlture forestière.

De la réserve. - J'ai déjà parlé de l'arantage fül y a pour certaines classes de propriétaires 
à constituer une réserve, et des facilités qu'orrient les forèts pour l'établissement de cette mesure de prévoyance. Le régime de la futaie donne, à cet égard, bien plus de facilités que celui du taillis: on pourrait, à la rigueur, avoir un coin de forêt réservé pour les besoins imprévus; mais il est bien plus avantageux de faire un prélèvement sur la production annuelle, car une fois les ressources de la réserve assise sur le terrain exploitées, il faudrait attendre sourent fort longtemps que la nature les ait reconstituées. En formant un prélèvement de 10, 15, 25 p. $100 \mathrm{sur}$ le revenu, on satisfait à la condition la plus essentielle d'une réserve, celle de porter toujours sur des bois exploitables, et d'ètre toujours disponible.

Parmi les deux éléments du revenu d'une futaie, il est complètement inutile de fiare un prélèrement sur les produits intermédiaires des coupes d'éclair cie, car ces coupes ont surtout un but cultural donf il ne faut pas les distraire, et elles produisent trop peu d'argent pour être une ressource sérieuse. C'esi sur le volume des coupes principales que se fait la réserve: sur les 12,000 mètres cuhes qui sont destinés à fournir les coupes principales, on prélèvera 25 p. 100 (si l'on éralue à ce chiffre les besoins imprérus), soit 3000 mètres cubes. Le restant, $900 \mathrm{C}$ mètres cubes, fournira tous les ans une coupe ordinaire de 450 mètres cubes. 
Les 3000 metres cubes de réserve seront toujours à la disposition du propriétaire, sauf les exigences du repeuplement de la première affectation ; ils lui fourniront une ressource dont la moyenne est de 150 mètres cubes par an, et qui pourra ètre exploitée daus les ringt années de la période, sauf à reconstituer pareille réserve quand on entrera dans la deuxième affectation en 1880 .

Compte d'aménagement. - En suivant exactement le plan d'exploitation, le propriétaire sera toujours assuré de ne jamais entamer le malériel de son exploitation, l'outillage de sa forèt; mais il arrive presque toujours que les exigences des semis font hater ou retarder les coupes sur telle ou telle parcelle; que des éclaircies ne sont point faites parce qu'elles donneraient des produits insuffisants, ou que des coupes d'importance diverse sort prises sur le compte de la réserre. Ces circonstances font naitre dans les futaies, bien plus que dans les taillis, un besoin impérieux de tenir exaciement la compl.. tabilité de la forèt en ce qui concerne le compte capital.

Le registre de contrôle ou plutiot le compte d'aménagement se tiendra comme pour los taillis: mais il sera tenu un feuillet séparé pour chacune des suites de coupes que comporte l'aménagement. Un compte sera ainsi ouvert à chaque parcelle, ot les volumes 
pris sur la réserve seront inscrits en rouge pour éviter la confusion.

Voici un compte d'aménagement dressé pour la forèt qui nous sert d'exemple, en supposant que l'aménagoment ait été fait en 1860 :

\section{Compte d'aménagement.}

PREMIIÈRE PÉRIODE, DE 1860 à 1879.

A. - Coupeg par volume.

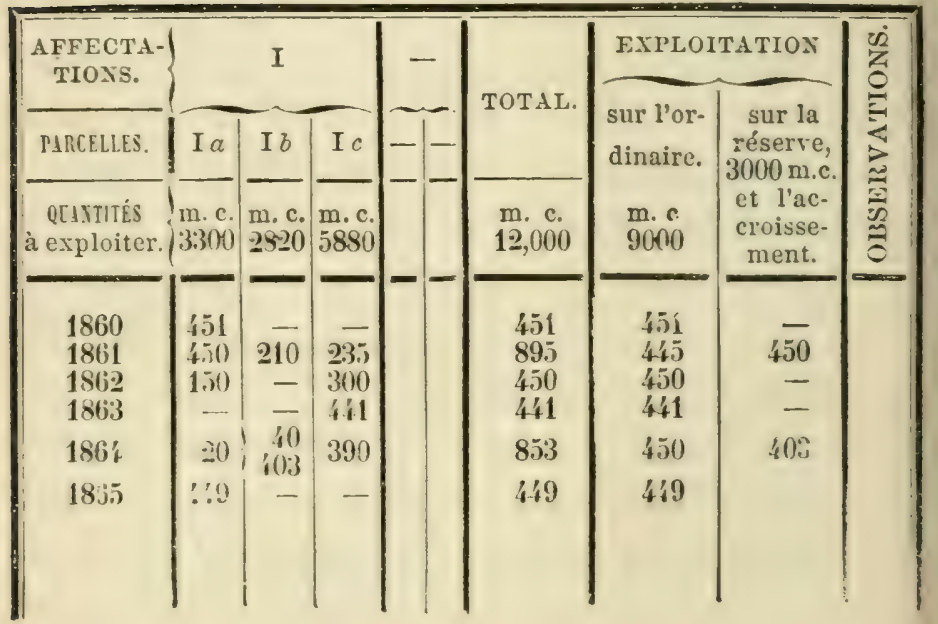


B. - Coupes par contenance.

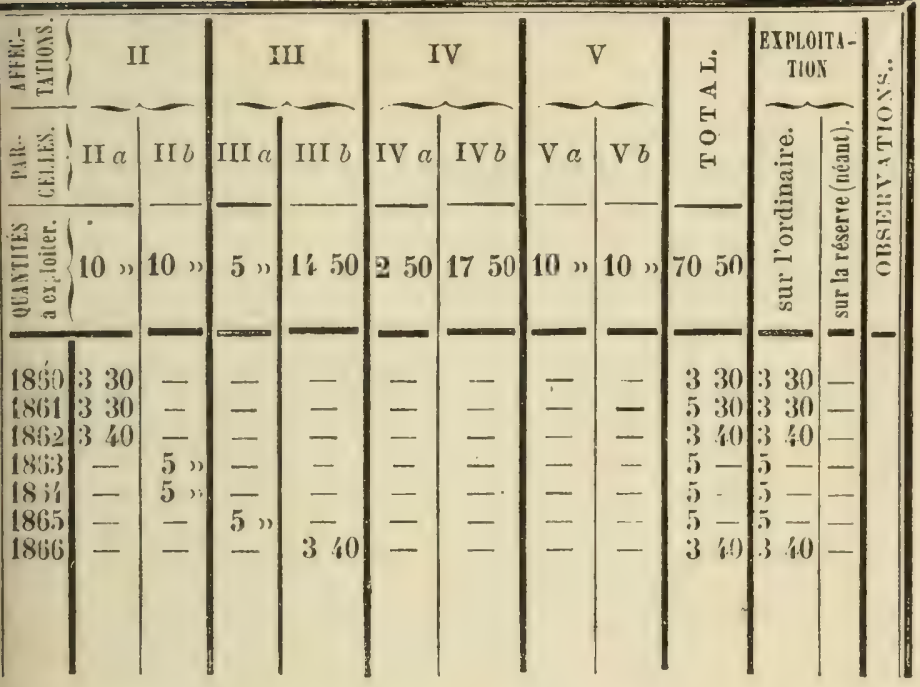

Ces comptes montrent qu'en 1861 on a exploité 450 mères cubes sur la réserve dans I $a$, et 445 mètres cubes sur ordinaire, en deux lots, dans I $b$ et I $c$. En 1862, la coupe rinaire a été formée par deux lots: 150 mètres cubes dans $a$ et 235 mètres cubes dans I $c$; c1l 1853, un seul lot de 141 mètres cubes dans I $c$; en 1861, exploitation sur la réserve de 403 mitres cubes dans I b. La coupe ordinaire a été formé par 20 mètres cubes de chablis dans I $a, 40$ mètres cubes dans I $b$ et par un lot de 390 mètres cubes dans I $c$. Quant aux éclaircies, la moyemne de la coupe annuelle est de 4 hectares; on voit qu'on a voulu enfermer les coupes dans les limites des parcelles de manière à ne pas avoir a coupe d'une année assise dans deux parcelles et à marcher cependant ni plus ni moins vite que si on avait rigoneuscment exploité 4 hectares par an a ansi la parcelle II $\alpha$, de 10 hectares, a fourni trois coupes de $3^{\text {hh }}, 30$ pour les trois ammées $1860,1861,1862$; la parcelle II b, de même conte. 
nance, n'a été divisée qu'en deux coupes de jo hectares pour 1863 et 1864 , de maniere à parcourir les 20 liectares en cinq ans, comme l'auraient fait cinq coupes de 4 hectares, etc.

\section{B.}

Aménagement des futaies dont le matériel d'exploitation est constitué, mais irrégulièrement disposé.

Au point de rue de la production, il importe peu que les cantons de forèt qui constituent le matériel d'exploitation soient disposés dans un ordre régulier' il peut mème arriver qu'un propriétaire possède plusieurs bois dans direrses communes et ru'il les exploite suivant un plan déterminé. Mais, au point de vue des facilités d'administration el de garde, au point de vue de la vente des produits, la régularité est toujours très-arantageuse. Ce qui n'a pas d'inconvénient pour un bois de particulier peut en avoir pour une forèt domaniale qui doit alimenter un certain bassin de consummation, ou pour une forèt communale qui doit fournir des produits à la portée des habitants. Les efforts de l'aménagiste devront done toujour's tendre à ramener cette régularité desirable dans la constitution dı matériel d'exploitatation : on pourra ne pas craindre d'exploiter certaines parcelles arant leur àge et d'en retarder d'autres; les sacrifices faits sur un point sont le plus sourent compensis par la plus-ralue acquise sur d'autres.

La réserve faite sur le volume pour les hesoins 
imprérus peut servir aussi a parer aux diminutions accidentelles qui résulteront de ces sacrifices. Les combinaisons d'aménagement sont nombreuses et fécondes, et parmi celles qui sont en usage, une des plus utiles est celle qu'offre, dans les grandes forèts, l'établissement de différentes séries d'exploitation. On entend par ce mot une partie de forêt considérée comme un tout séparé, indépendant, destiné it être soumis à un plan particulier d'exploitation et it fournir par conséquent des produits anmuels.

Dans une masse considérable de forèt, il sera presque toujours facile de classer dans telle ou telle sẻrie les parcelles qui doirent former le matériel d'exploitation et de les grouper d'une manière assez régulière pour atténuer les sacrifices d'exploitabilité.

La division des grandes forèts en séries d'exploitation pro!luit encore un autre arantage, c'est de permettre de proportionner entre eux les différents éléments qui entrent dans la constitution du matériel d'exploitation: âge, contenance, étal de végétation. Los différences dans les conditions de la végétation et, par conséquent, dans l'élat des peuplements, qui s'opposent souvent à la formation des affectations de contenance égale, peurent se grouper dans une même séric, et la production générale de la forèt n'en est que mieux soutenue, mieux maintenue à un niveau 
AMÉNAGEMENT DES FUTAIES.

constant. Cela arrivera, par exemple, si dans une vallée couverte de forêts on peut constituer en séries différentes le plateau, le versant méridional et le versant septentrional. Les affectations de contenance égale qui seront créées dans chacune de ces séries seront alors équiproductives autant que la nature des choses le comporte.

\section{$\S 2$.}

Futries dont le matériel d'exploitatıon est surabondant.

Considérations générales. - Il peut arriver nonseulement que, dans la forèt à aménager, certaines parcelles soient d'un âģe tel que la marche des exploitations ne les atteindrait qu'à un âge trop avancé pour fournir de bons produits, mais encore que le volume des bois existant sur la forèt soit trop considérable pour le but que s'est proposé le propriẻtaire, c'est-à-dire pour l'àge d'exploitabilité choisi. Il est d'aussi mauraise administration de chercher à produire des bois d'une certaine nature, des étais de mines, des charpentes, par exemple, avec un matériel trop riche que de faire de l'agriculture avec des bâtiments somptueux et un outillage de luxe. Un propriétaire de forêt $a-t-i l$ pensé qu'il aurait le débit le plus avantageux de ses bois en les coupant a cent vingt ans? A-t-il, en conséquence, 
fait de cet àge la base de son système d'exploitation? Il lui faut pour son exploitation un matérielbois de un à cent ringt ans; tout ce qui dépasse (d'une manière notable s'entend) est du luxe et de la surabondance, dont l'effet est de diminuer considérablement la rente déjà fort basse qu'il retire du capital engagé.

Il n'est pas sans utilitẻ de faire remarquer que cutte situation ne se présentera pour l'État que dans des cas très-rares et très-exceptionnels. Les intérêts de l'État propriẻtaire de forêts ne sont pas autres que ceux du consommateur; ses besoins, ceux du travail national. Or, un arbre fournit d'autant plus de marchandises utiles qu'il est plus gros et plus voisin de sa maturité. D'un autre còté, les bonnes routes, les chemins de fer et les canaux tendent de plus en plus à répartir dans tout le pays les produits qui, jusqu'alors, restaient sur place à cause du prix des transports. La surabondance d'une forêt compense la pénurie d'une autre, et c'est dans le cas seulement où la vitalité de certains massifs serait compromise par un trop long retard dans l'exploitation cu'il pour'a exister des forèts domaniales à capital surabondant.

Aménagement. - On conçoit que dans les fu * taies dont le matériel dépasse ce qui est nécessaire pour l'exploitabilité choisie, il y a deux groupes paralleles d'exploitation: l'un dépendant de la marche 
nol'male de l'aménagement, attaquant les affectations au fur et à mesure que leur tour arrivera; l'autre ayant pour but de chercher à réduire le matériel et à le ramener à son échelle normale. Il n'est nul besion de rérolution ou de période transitoire, car la marche normale de l'aménagement doit être attaquée immédiatement el concurremment arec ces coupes de réduction. Ge mot n'a pasici de sens cultural, etn'est employé que pour indiquer le but à atteindre, car ces coupes seront, selon la nature des peplements, ou des extractions de vieux bois, ou des coupes de régularisation tendant à ramenèr des peuplements irréguliers à un état plus homogène. Aussi, pour ne pas faire de néologisme dans une science où l'on est entraîné sourent à confondre la nomenclature culturale arec celle de l'aménagement, on distingue tout simplement sur le plan d'exploitation les coupes qui dépendent de cet ordre d'idées, en les classant sous le titre général de produits en dehors de la marche normale de l'aménagement, ou plus simplement produits anomarm.

Je dois faire une obserration. C'est que, malgré la diversité des opérations forestières, malgré toutes les ressources que présente la régétation des bois, il n'est jamais possible de ramener un ensemble de peuplements à une échelle d'àges normale et convnablement graduée. Les règles cuiturales, les cxi- 
gences de la régétation, la nature dos essences et mille autres circonstances sont autant d'éléments qui entrent dans la question et qui sopposent à la solution complèce du problème. Il vaut mieux se résignel à un peu de luxe dans une exploitation de futaie que de compromettre la longévité ou la bonne végétation d'un massif par des extractions d'arbres ou des opirations sans caractère cultural. En cette matière, le luxe n'est, en réalité, que de l'épargne et une sage appréciation des besoins de l'avenir.

Dans l'état actuel de nos fulaies, exploitées jadis sans ordre, sans but et sans autre reggle que le caprice, les cas qui se préscnteront à l'aménagiste se-• ront très-nombreux et très-différen's, mais aussi les combinaisons d'aménagement sont très-rariées. Je ne puis indiquer, dans ces simples notions, toutes celles que la pratique el l'expérience ont apportées au domaine de la science forestière: l'cmploi tempo-. raire du taillis dans les Joois feuillus, lintroduction du jardinage, mode dont je dirai plus tard un mot, seront sourent usités dans les combinaisons d'aménagement.

Je ne citerai que deux exemples de ces combinaisons, mais je dois dire arpararant qu'clles sont toutes subordomnés a une rìgle générale, yui est celle-ci :

Quels que soient l'irrégularilé et l’àge des massifs 
qui forment la forèt, on doit toujours, une fois que le travail analytique du parcellaire est fait, établir le plan général d'exploitation, c'est-à-dire créer les affectations et les délimiter sur le terrain. Ce tracé des affectations est, en effet, le cadre général auquel doivent s'adapter toutes les opérations à faire dans la forèt: il imprime aux coupes une marche et un caractère conformes au but à atteindre; il est la base fondamentale de l'exploitation en futaie.

Une fois ce plan général établi, il sera toujours facile de déterminer les coupes qui dépendent de sa marche naturelle et celles qui sont destinées, tout en se conformant aux règles culturales, à réduire le matériel existant et à le ramener à une échelle d'ìges plus régulière. Ces coupes pourront presque toujours se faire dans la première période de l'aménagement, et il ne sera utile d'en réserver la complète terminaison pour la seconde période que si leur volume était trop considérable pour les besoins de la consommation. Elle ne seront distinguées, sur le plan d'exploitation spécial à la première période, que par un simple chapitre; leur possibilité, c'est-àdire leur volume annuel, sera confondu avec celui des coupes provenant de la marche normale de l'aménagement, afin de laisser aux agents chargés de l'exécution toute la latitude que comporte une bonne culture forestière. 
Premier exemple. - Nos appliquerons ces principes à un premier exemple particulier: une forêt de trois cent soixante hectares destinée à ètre exploitée à cent cinquante ans, dont la période de régénération est de vingt-cinq ans, et dont le peuplement est le suivant :

A. - 15 hect. semis sous le couvert de vieux hêtres, de 15 et 120 ans.

B. - 285 " jeune futaie exploitable de sapin, peu régulière avec bors d'âges divers, variant de 70 à 120 ans.

C. - 20 " perchis de hêtre régulier, de 40 ans.

D. - 40 " mélanges de tous âges, fourrés perchis et vieux bois, hêtre et sapin, de 20 à 130 ans.

360 hectares.

La combinaison d'aménagement pourra être de créer six affectations de contenance égale correspondant à six périodes de vingt-cinq ans, de régénérer en première période la Ire affectation, de créer des jeunes bois en régénérant également la VIe affectation, pour l'exploiter deux fois dans le cour's de la révolution, et de soumettre le restant de la parcelle $B$ à des éclaircies dont le but sera d'enlever les bois trop âgẻs sans toutefois interrompre le massif. 
A MENAGEMENT DES FUTAIES.

\section{Plan génèxal d'exploitation.}

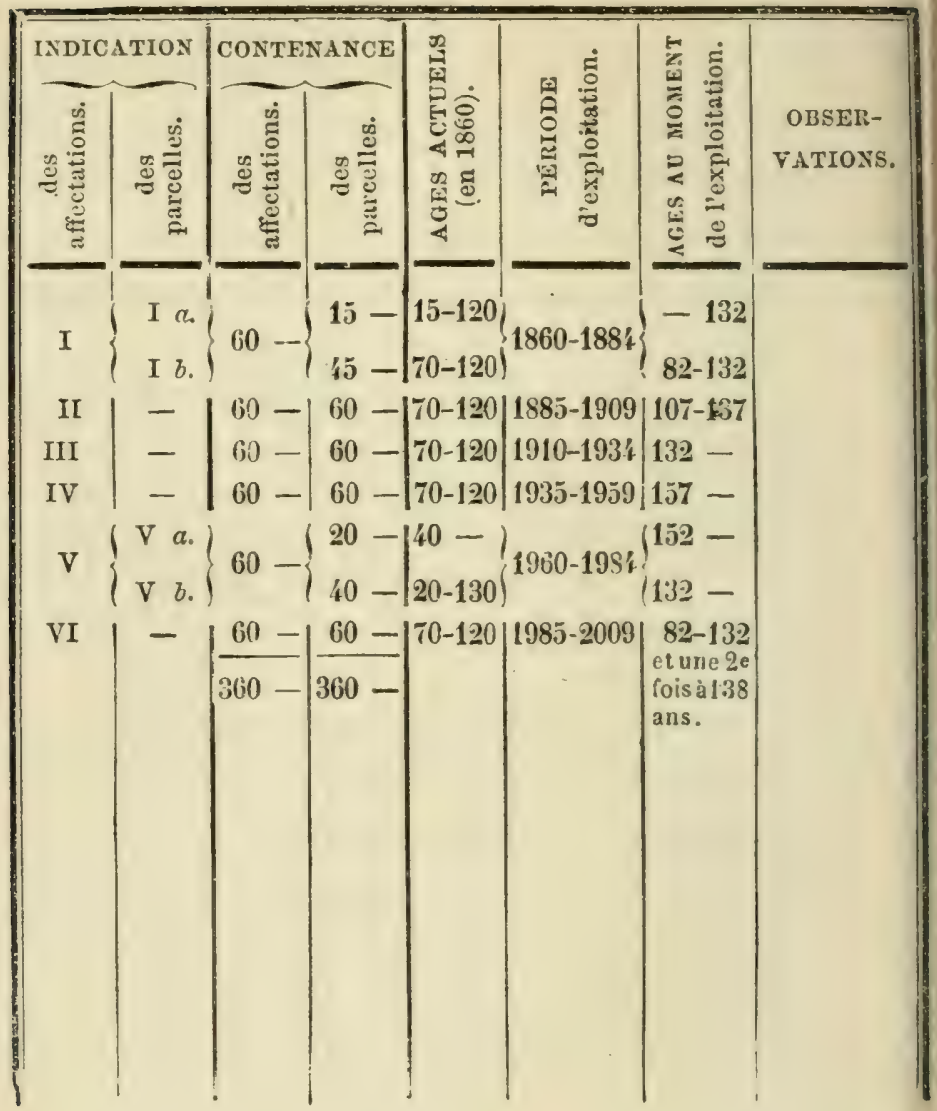


FUTAIES PLEINES.

\section{Plan spécial d'exploitation pour la premiére période (1860-1879).}

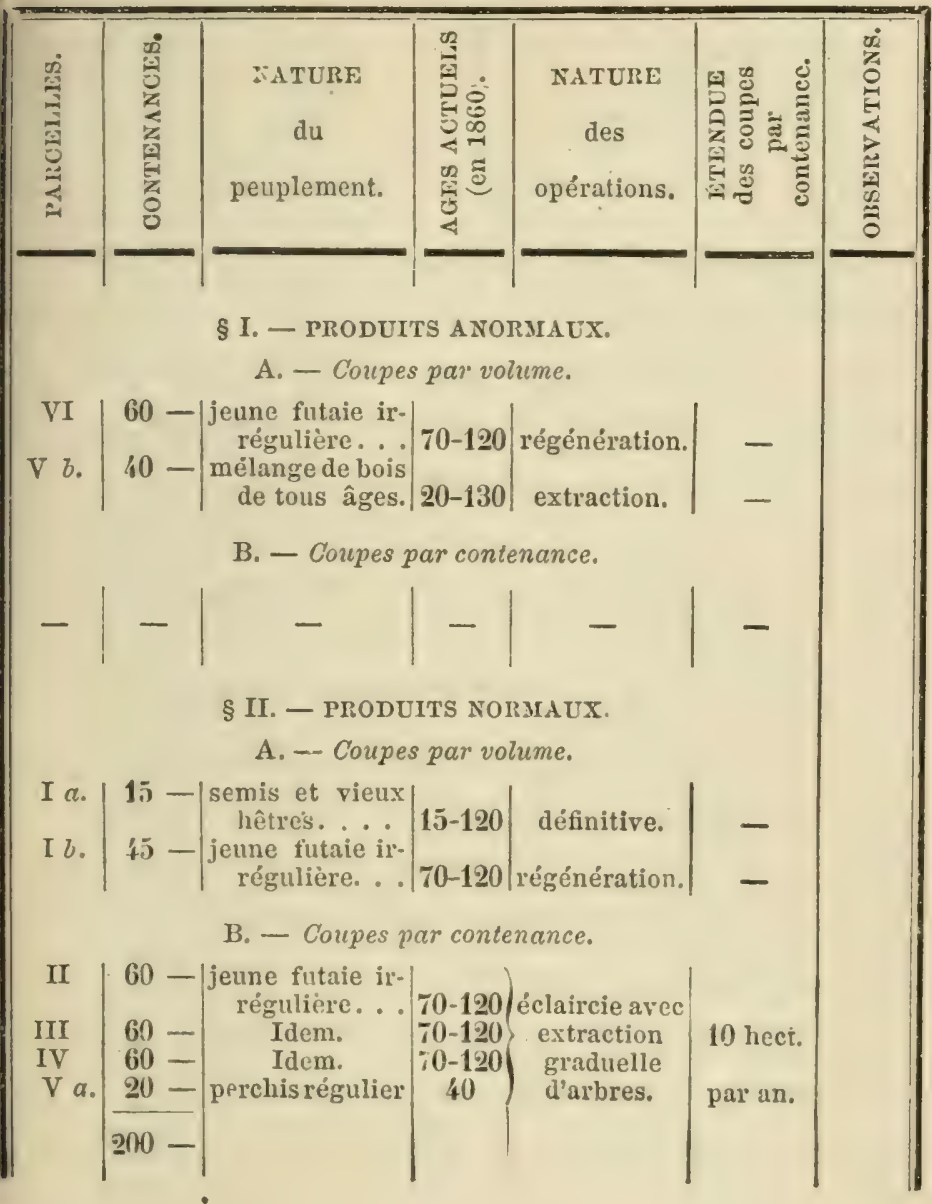


La possibilité de coupes par volume a été évaluce ainsi :

VI présente. . . . 24,090 mètres cubcs.

$$
\begin{aligned}
& \mathrm{V} b \quad \text { " . . . . 5,410 " } \\
& \text { I } a \quad " \quad . . . . . .3,360 \quad \text {. } \\
& \text { I } b \quad \text { " . . . . } 17,940 \text { " }
\end{aligned}
$$

La coupe annuelle sera donc de un vingt-cinquième, soit . . 2,032 "

$\mathrm{Du}$ volume extraordinaire. - Il est évident que dans une pareille forêt il y a un volume extraordinaire qui ne se représentera plus à la seconde période et qu'il est toujours fort utile d'évaluer. Car, si c'est une forèt de l'État, il importe de porter ce produit aux ressources extraordinaires; si c'est une forèt communale, il est utile de renseigner la commune sur cette partie de la production qu'elle pourra employer à un travail d'utilité publique; si enfin la forèt appartient à un particulier, ces produits extraordinaires pourront donner lieu, soit à un remploi, soit à une reprise de communauté, soit à un arrangement avec un nu-propriétaire ou un usufruitier.

Pour évaluer ce volume extraordinaire, il ne faut pas songer à séparer les produits qui dépendent de la marche normale de l'aménagrement de ceux qui sont recueillis en dehors de cette marche, et à calculer pour chaque nature de produits une possibilité spéciale. 
Produits normaux.

$1 \mathrm{a}$. . . . 3,360mec

I $b \cdot \cdots \cdot 17,940$

$$
\text { Total. . } \overline{21,300}
$$

Possibilité, un vingt-cinquième :
Produits anormaux.

$$
\begin{aligned}
& \mathrm{Vb} \text {. . . . } 5,410^{\text {mce }} \\
& \text { VI . . . } 24,090 \\
& \text { Total. . 29,500 }
\end{aligned}
$$

Possibilité, un

vingt-cinquième: 1,180

Ce procédé, qui aurait pour effet de donner au volume extraordinaire une assiette fixe sur le terrain et de gèner parfois les régisseurs pour la rente de certains produits, ne serait point exact. Il ne faut pas confondre, en effet, les produits anormaux arec le volume extraordinaire. Celui-ci ne peut se rencontrer que dans les forêts dont le matériel d'exploitation est surabondant; il ne saurait exister dans celles dont le matèriel est insuffisant; or, dans l'aménagement de ces dernières, il arrive souvent que l'on glane en dehors de l'affectation en tour certains produits dans le but de rendre l'attente moins pénible au propriétaire. Dans les forèts dont le matériel est surabondant, il peut même arriver que la première affectation soit déjà fortement entamée, qu'elle présente fort peu de produits (la forèt dont nous nous occupons en est un exemple), et si l'on considérait les produits recueillis en dehors de la marche normale des affectations comme un volume extraordinaire, on serait conduit à avoir de celui-ci une illée complétement fausse. 
Ces différents termes ont chacun leur valeur: l'expression ("produits normaux et anormaux) caractérise les emplicements où sont recueillis les produits pendant la première période qui a presque toujours un caractère préparatoire ou transitoire.

Les mots "prorluits ordinaires et extraordmaires») s'appliquent, d'une part au revenu habituel de la forèt pour l'àge de l'exploitabilité choisie, et, d'autre part, à tout ce qui, dépassant ce revenu, se prend sur un capital préexistant pendant un laps de temps.

La mesure de cette portion de la production dépend de l'ìge choisi pour l'exploitabilité, mais ne saurait dépendre de la combinaison des parcelles dans le cadre de l'aménagement. La seule manière de mesurer ce volume extraordinaire est de comparer la masse totale des produits à recueillir à ce que la forêt pourrait fournir si son matériel était régulièrement constitué en vue de l'âge choisi pour l'exploitation de la forèt. On évaluera ce que peut produire un hectare àgé de 150 ans dans leś conditions moyennes de la régétation de la forèt. C'est une question de pratique et d'expérience dont la solution sera precque toujours aidée par des types choisis dans la furèt mème ou dans les forêts voisines. Si ce chiffre est de 600 mètres cubes à l'hectare pour l'exemple qui uous occupe, on le multipliera par la contenance de l'affectation et on comparera le résul- 
at an rolume tolal à recueillir; l'excédant représen:cra le volume extraordinaire avec l'approximation jui est dans la nature des choses.

Tolume total à exploiter

aroil : Produits normaux 21,300mec

I Produits anormaux 29,500 $\}$. 50,800

Pruduction ordinaire évaluée à 600 mètres cubes

par hectare $600 \times 60=$. . . . 36,000

Le volume extraordinaire est de. . . 14,800

La possibilité ordinaire est fixée ì. . . . . 1,440

Le volume extraordinaire présente une ressource dont la moyemne amuelle est de. . 1,080

Il arrivera souvent que ce volume extraordinaire sera constitué en réserve par le propriétaire et affecté par lui à la satisfiction des besoins imprévus qui peuvent se présenter dans les vingt-cinq années de la première période. Mais si un particulier est obligé de faire remploi de ces 1'4,800 mètres cubes et reut cependant se constituer une réserve sur le revenu; si une commune les affecte à un trarail d'utilité publique et si elle reut cependant avoir son quart de réserve et ne distribuer aux habitants que les $3 / 4$ de la production normale de la forcit, il y aura lieu de farire une distinction entre les compos ordinaires, les coupes de réserve et les coupes extraordinaires. Le plan d'exploitation portera, par exemple, dans la colonne d'obserration, la mention suivante: 
Si c est une forêt communale arec çuart de réserve :

Volume total à exploiter . . . . . . 50,800 mec

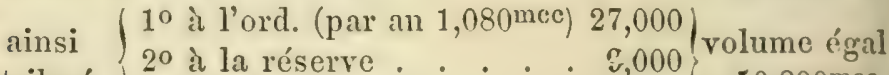
attribué : $\left.\mid \begin{array}{l}3 \text { à l'extraordinaire . . . 14,800 }\end{array}\right\} \quad 50,800^{\mathrm{mec}}$

Si c'est une forèt domaniale:

Volume total à exploiter . . . . . . $50,800^{\text {mec }}$ ainsi $\left\{\begin{array}{l}\left.1^{\circ} \text { à l'ord. (par an } 1,440^{\mathrm{mec}}\right) 36,000 \\ 2^{\circ} \text { à la réserve, l'accroissement. }\end{array}\right\}$ attribué: $\left\{\begin{array}{l}2^{\circ} \text { à l'extraordinaire . . 14,800 }\end{array}\right\} 50,800^{\text {mec }}$

Compte d'aménagement. - On tient dans ce cas le compte d'aménagement, comme nous l'avons indiqué; sa formule s'adapte à toutes les circonstances. Il suffit d'ajouter une colonne supplémentaire pour indiquer les exploitations qui ont été faites sur le volume extraordinaire; on lui donne plus de clarté en employant l'encre noire pour les volumes pris sur l'ordinaire, rouge pour les volumes pris sur la réserve, et bleue pour ceux attribués à l'extraordinaire.

C'est surtout pour les forêts communales que celte comptabilité a une grande importance; comment résister aux demandes incessantes d'une commune si on ne peut lui montrer, par des comptes bien tenus, oủ elle en est de sa réserve et de ses ressources? L'aménagement n'est-il pas le contrat de la gestion de la forêt, interrenu entre le propriétaire et le ser- 
vice public chargé de la régeie, contrat dont l'exécution peut ètre réclamée judiciairement devant les tribunaux administratifs? Faut-il, en cas de contestations, s'exposer à avoir les mains vides de justifications et de preuves?

Deuxième exemple. - Ln cas assez emharrassant est celui de la forèt de H..., qui, sur 500 hectares, présente un massif de sapins pur très-régulier et àgé uniformément de cent quatre-ringts ans.

Si, dans une pareille circonstance, on exploitait toute la surface dans une révolution transitoire de ringt ou de trente ans, par exemple, on laisserait, au bout de ce temps, la forêt peuplée de bois de un à trente ans, et le public consommateur serait privé de bois de serrice pendant un temps fort long, au bout duquel la mème situation se représenterait. On s'exposerait d'ailleur's à rendre à ril prix, pendant toute la période transitoire, des produits précieux exploités sur le mème point en trop grande quantité.

La combinaison suirante peut ètre adoptée: on dirise la forèt en six affectations correspondant it des périodes de trente ans, on régénère la Ire en première période, on prescrit des coupes préparatoires à l'ensemencement sur la' IIe et on introduit dans les.IIIe, IVe, Ve et VIe des coupes de jardinage par contenance et arec une rotalion de cinq ans. Cette combinaison, qui est loin d'ètre parfaite, parce qu'elle 
introduit dans la forèt un mode de culture critiqué par bien des auteurs, a du moins l'arantage de conserver le sol couvert sur toute la forêt, de répartir sur la durée de la révolution une notable partic du volume de la sapinière et de satisfaire enfin au but principal de tout aménagement, qui est de préparer pour la seconde révolution un matériel d'exploitation complet et régulier

\section{$\S 3$.}

Futaies dont le matériel d'exploitation n'est pas constitué d'une manière suffisante.

Généralités. - Des exploitations abusives ont pu ravager une forèt jadis exploitée en futaie pleine, à cent quarante ans par exemple; une liquiảation de droits d'usage, un partage de famille ont pu faire passer en diverses mains son matériel d'exploitation; celui-ci n'est plus constitué d'une manière suffisante, et cependant le propriétaire est toujours décidé à exploiter la forêt à cent quarante ans. A ne considérer que les termes mêmes de la question, le probleme est insoluble: il est tout aussi impossible d'exploiter immédiatement à cent quarante ans que de faire de l'agriculture quand la ferme n'est pas construite. Puisque le capital n'est pas constitué, il y aura forcément un certain temıs pendant lequel 
on n'exploitera pas entièrement à cet ìge; mais si l'on se reporte à la définition d'après laquelle l'aménagement a pour but de déterminer le matériel d'exploitation, de lui préparer un cadre de telle sorte que les exploitations ne viennent pas à l'entamer, on verra que le probleme est non-seulement trés-suluble, mais qu'il a un but nettement tracé, qui est celui-ci :

Diterminer, pour le matériel d'exploitation, an cadie dressé en vue du terme d'exploitabilité choisie, et fixer la nature et la marche des exploitations qu'il sera possible de faire pendant. le temps que la nature mettra à constituer le matériel d'exploitation.

Il y aura done forcément, dans l'aménagement, leux phases bien distinctes : l'une transitoire, dans laquelle on fournira des produits qui ne satisferont pas encore entièrement au but que le propriétaire s'est proposé, et l'autre, définitive, qui lirrera à l'exploilation des produits correspondant à l'exploitabilité choisie.

Ciette époque de transition durera plus ou moins longtemps, selon les circonstances; elle pourra n'ètre qu'une phase temporaire, dans un aménagement défnitif, ou une période transitoire précédant l'aménagement, ou enfin une rérolution de conversivn. Les car qui peurent se présenter sont, en eflet, 
tres-nombreus, et nous derons, avant d'aller plus loin, établir une distinction inportante:

Parmi les forèts dont le matériel n'est pas assez complet pour se prêter à l'exploitation en futaie, à l'àge que le propriétaire s'est fixé, on peut avoir à aménager :

A. Des forèts qui ont toujours été traitées en futaie ou qui ne se prêtent, comme celles d'essences résineuses, qu'à ce mode de traitement, mais qui ont été dérastées par des exploitations abusives : c'est ce que les ancieus forestiers appelaient reconduction en futaie".

B. Des forêts qui ont toujoursété traitées en taillis et qu'il faut convertir en futaie.

Je m'occuperai d'abord de la première catégorje, et je parlerai ensuite des conversions, qui ont des rigles et des combinaisons toutes particulières.

\section{A.}

Reconductions en futaies pleines.

Je suppose quele propriétaire ait fixé la rérolution, c'est-à-dire qu’il se soit décidé à produire des bois d'un àge déterminé, à cent ringt ans, par exemple; je suppose également que le parcellaire de la forct

1 Cette expression n'est plus usitce dans le limgage forestier; nous l'employons, non pour la faire revivre, mais pour caractériser plus nettement des situations qui se rencontrent sourent dans les régions montagncuses. 
ait ètẻ fait et ant montré que les différents massifs qui compascne la forè! ne présentent pas la gradation d'igges de un i cent vinght ans, nécessaire pour l'exploitation regulière de la forêt: il y aura forcément une époque de transition arant de commencer la marche normale de l'exploitation à cent vingt ans. Dans celle époque de transition, on pourra convenir d'exploiter cer'aines pariies prématurément, ou bien chercler à glaner des produits sur toute l'étendue de la forc̀t; celte époque sera, comme nous allons le roir bientòt, ou une période transitoire spéciale anlérieure à l'aménagement, ou une simple phase dans l'aménagement définitif.

Mais, quel que soit son caractère, il sera toujours facile d'en déterminer la durée; ce sera vingt ans, quarante ans, selon l'âge des loois les plus âgés et leur classement dans le plan d'exploitation. Celte seule considération indique déjà la nécessité de tracer sur le terrain le cadre des affectacions. On en a tous les Ciements: la durie de la période de régénération qui dévend du climat et de la nature des essences, et le parcellaire qui a été fait pour connaître les rescources de la for'èt.

Il faut donc établir, arant tout, le plan de l'aménagement définitif. Cela est nécessaire, nonseulement par les considerations que je viens d'in-diquer, mais encore par celles que j’ai expliquée. 
au chapitre des taillis qui correspond à celui-ci (page 61).

Une fois le plan général d'exploitation établi, c'est-à-dire l'aménagement définitif assis sur le terrain, il y a deux manières d'opérer:

La première consiste à déterminer la durée de la période transitoire d'après les exigences des âges et à classer les bois les plus àgés dans une première affectation d'une révolution qui sera précédée de la période d'attente.

Dans la seconde manière, au lieli de classer en première affectation les bois les plus âgés qui forment ce que les forestiers appellent la tête d'aménagement, et de convenir qu'on ne les exploitera qu'au bout d'une période d'attente de vingi ou quarante ans, on recule leur numéro d'affectation, et on lesclasse en deuxième ou en troisième, selon le temps qu'ils doivent attendre. La période d'attente devient ainsi égale à une ou deux périodes de régénération. La première ou les premières périodes de l'aménagement sont des périodes de la phase de transition dont nous avons parlé; on y achève la régénération de certaines parties commencées, ou l'on y exploite prématurément certaines autres, ou enfin on y cherche à glaner des produits sur toute l'étendue de la forêt et en dehors de la marche normale de l'aménagement. 
De ces deux modes d'opérer, le premier est géneral, en ce sens qu'on peut toujours l'appliquer; le second s'adresse à un cas particulier, mais d'une application très-fréquente, car, dans l'impossibilitẻ où l'on est de dèterminer d'une manière bien précise la durée des périodes de régénération et de transition, on peut très-souvent faire coïncider la durée de l'une et de l'autre. On a ainsi l'arantage d'entrer immédiatement dans la marche de l'aménagement définitif, avantage considérable, car on supprime le rouage d'une période transitoire spéciale, on obient une grande simplicité et on imprime à chaque coupe un caractère bien défini. Si, par exemple, une coupe d'extraction de vieux arbres doit ètre faite sur une parcelle de première affectation, nul forestier n'hésitcra à y roir une coupe définitive plus ou moins tardive; - si elle doit être faite en quatrième affectation, nul n'hésitera non plus sur la manière dont la coupe doit être conduite, sur la catégorie des arbres à enlever, car on tiavaille dans le cadie même de l'aménagement. Arec une période transitoire spéciale de quarante ans, par exemple, on est obligé, pour connaitre le caractére de cliaque coupe, de fuire un calcul fort simple, sans doute, mais qui peut amener des erreurs ou des omissions dont l'effet est presque toujours une fante grare pour la marche de l'aménagenent. Enfin, daris les gestions de forèls 
importantes, on a l'arantage d'introduire dans la régie une marche uniforme qui se plie au control. administratif.

La production des premières périodes de l'aménagement se compose, ici comme dans les cas précédents, de deux élements: les produits provenant de la mar'he normale de l'aménagement, qui peuvent se réduire à fort peu de chose, et les produits provenant des coupes faites en dehors de cette marche normale: ceux-ci se recueillent un peu parlout et forment la production essentielle de la période d'attente.

Des exemples feront mieux comprendre une théorie que j’ai essayé de généłaliser.

Quand j'ai parlé de la constitution du capital d'exploitation des forèts (page 22), j'ai insisté sur ce que l'on doit entendre par ce mot: il faut que nonseulement la forèt présente en volume le matérielbois correspondant à une échelle d'àges de un à cent vingt ans, mais encore que ce volume soit réparti sur des peuplements d'àges gradués convenablement.

Supposez, par exemple, une forèt de 120 hectares peuplée de perchis réguliers renant d'ètre éclaircis, et ìgés uniformément de soixante ans ; on aura bien un capital-bois consitité en ce sens que le rolume. fourni par une échelle de peuplements gradués de un à cent vingi ans est sensiblement représenté par 
ur peup!ement uniforme de l'àge moyen de soixante ans, mais cette forêt ne sera pas constituée pour une exploitation immédiate en futaie à l'âge de cent vingt ans, car les bois les plus vieux n'ont pas cet âge et ne sont même pas arrivés à l'époque où ils donnent des semences d'une manière régulière.

Supposez, comme autre exemple, une forêt de 120 hectares présentant un petit massif de jeune futaie de quatre-vingts ans, et 100 hectares de jeunes bois de vingt ans, surmontés de grands arbres plus ou moins nombreux (jeunesses - coupes définitives à faire), voilà une forèt dont le matériel d'exploitation n'esi constitué ni par le volume ni par la gradation des âges.

Pour ne pas égarer le lecteur parmi les nombreux cas quc peuvent présenter les forêts irrégulières dont on se propose la reconduction, je m'en tiendrai à ces deux exemples •

Premer exearle. - 120 hectares de perchis de hêtre réguliers venant d'être éclaircis, âgés de soixante ans, et qu'on veut exploiter en futaie pleine à cent vingt ans.

$\mathrm{Si}$ on laissait vieillir pendante soixante ans ces peuplements, pour les amener à l'àge de l'exploitabilité.choisie, on aurait une forêt peuplée parlout de bois de cent vingt ans, ce qui aurait l'inconvénicnt tres-séricux de laisser le propriétaire sans produits 
(si ce n'est les coupes d'éclaircies) et d'avoir, à l'expiration de ceite longue période, un malériel surabondant, un luxe fort inutile et fort onéreux dans le capital d'exploitation.

Un grand propriétaire comme l'Etat pourrait combiner l'exploitation de cette forèt avec d'autres situées sur un autre point. Il pourrait ainsi laisser les peuplements vieillir en se contentant d'y faire quelques éclaircies; mais dans les conditions du'problème, c'est-à-dire d'une forêt unique et analoğue au type choisi ci-dessus, il peut être plus conforme aux intérêts du propriétaire de commencer immédiatement la régénération de la forèt. Or, à soixante ans, les hêtres ne donnent pas, en général, des semences d'une manière convenable pour la régénération par voie d'ensemencement naturel. Malgré la dépense que nécessitent les semis artificiels, il pourra y avoir encore un avantage notable à s'y résoudre, plutòt que de rester sans produits pendant longtemps et sans tirer aucun argent d'une forèt qui a dejjà une valeur considerable. On emploie alors la pratique des coupes d'ensemencement artificiel auxquelles on a donné le nom de coupes d'abri, parce qu'elles consistent is éclaircir les arbres de manière à obtenir un couvert convenable pour la germination des semis artificiels et à les enlever successivement et au fur et à mesure de la force des jeunes plants. 
En supposant que la période de régénération soit fisée à vingt ans, la combinaison d'aménagement pourra être la suivante :

Première Période. - Régénération, par voie artificielle, des Ire et VIe affectations.

Deuxième Période. - Regénération, par voie naturelle et artificielle, de la IIe affectation. Éclaircies par 1,20 de surface sur les IIIc, IVe et $V^{\mathrm{e}}$.

Troisième Période. - Régénération de la IIIe affectation par les voies naturelles qui sont dès lors acquises à la forèt. Éclaircies sur les autres affectations.

Quatrième Période. - Ainsi de suite.

\section{Plan général.}

I. 20 hect. 60 ans 1860 à $1879 \quad 70$ ans.

II. 20 " 60 " 1880 ̀े 189990 ”

III. 20 " 60 " 1900 à $1919 \quad 110$ "

IV. 20 ” 60 " 1920 à $1939 \quad 130$ ”

V. 20 " 60 " 1940 丸̀ $1959 \quad 150$ "

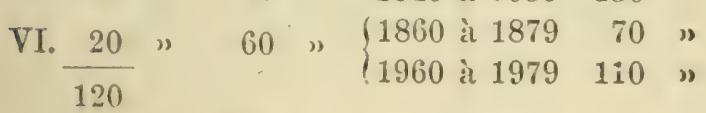

\section{Plan spécial.}

10 Produits en dehors de la marche normale de l'aménagement.

a) Coupes par volume.

VI. 20 hect. - 60 ans. - Coupes d'abri.

b) Coupes par contenance.

Néant. 
20 Produits dépendant de la marche normale de l'aménagement.

a) Coupes par volume.

I. 20 hect, - 60 ans. - Coupes d'abri.

b) Coupes par contenance.

Néant.

Devxième exemple. - Forêt de 120 hectares, présentant 20 hectares de jeune futaie de quatrevingts ans, et le reste en jeune bois de vingt ans surmonté de futries plus ou moins nombreuses en hêties et sapins. - Rérolution choisie, cent vingt ans. - Période, vingt ans.

Pour mieux fixer les idées, le parcellaire présente:

A. 10 hect. jeunes bois complets . . . . . 20 ans

B. 30 " jeunesses arec futaies d'âge inégal. 20 ì 130.

C. 20 " jeune futaie, hêtre et sapin. . . 80 ans.

D. 5 " gaulis et fourrés hêtre . . . . 20 "

E. 15 " gaulis arec bois épars de tous âges. 20 ì 130. F. 20 " gaulis et fourrés sapin . . . 20 ans.

G. 6 " coupes définitives à faire . . 20 et 130 . H. 14 " vides replantés. . . . . . 3 ans.

120

L'aménagement consistera à classer la parcelle $\mathrm{G}$ en IIIe affectation et à distribuer le reste de la contenance dans les autres affectations. Les deux premières périodes auront un caractère transitoire, et pendant leur durée on fournira des produits au moyen des arbres à extraire dans las jeunes peuple- 
ments. Dans la $1^{\text {re }}$ période, la production consistera dans l'extraction des arbres les plus vieux et dans les coupes définitives. On aura soin de ménager ces ressources pour avoir des produits suffisants pendant la seconde période, à laquelle on r'éservera les éclaircies, les coupes préparatoires à l'ensemencement et les extractions des bois qui ont pu altendre sans trop gèner les peuplements. A la troisieme période seulement on attaquera le petit massif de futaie actuellement âgé de quatre-vinğts ans; ce n'est qu'à la quatrième période que l'on commencera la régénération des jeunes bois qui forment actuellement l'ensemble de la forèt.

Plan général.

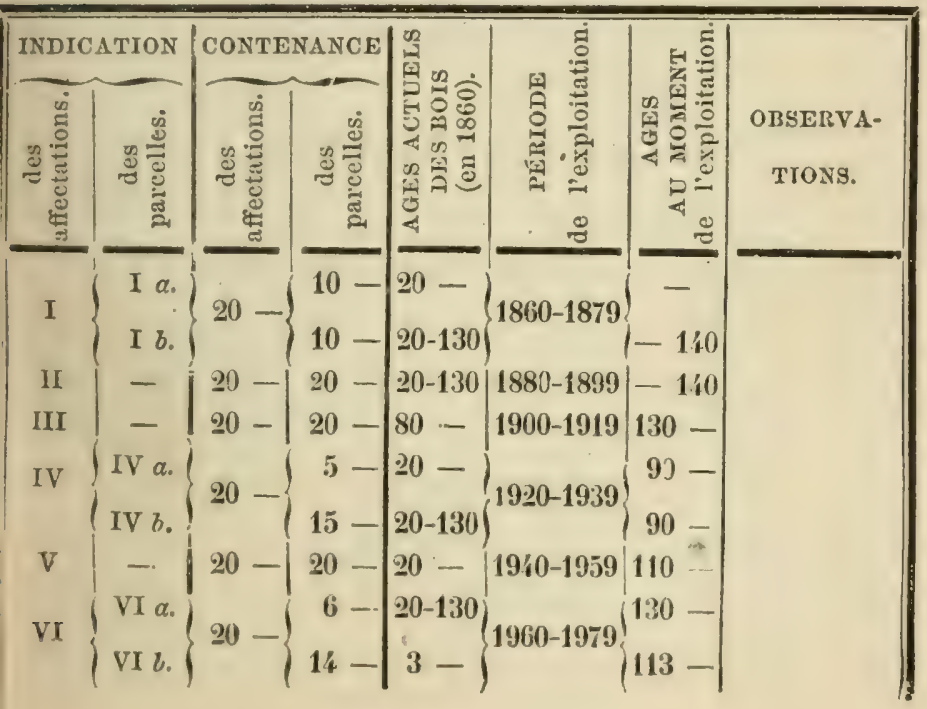


Plan spécial. - 1860-1879.

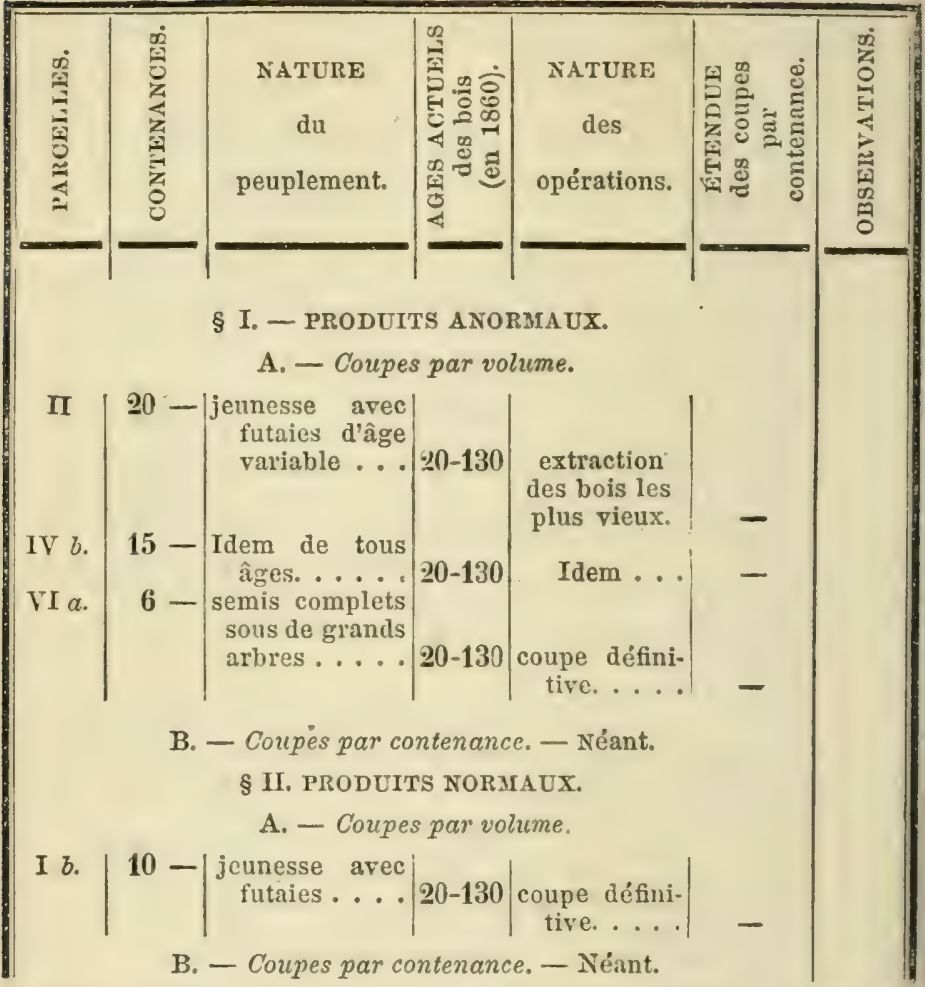

Possibilité. - Le matériel à réaliser dans la première période est de:

$$
\begin{aligned}
& \text { sur II } \\
& \text { IV } b . \cdots 930
\end{aligned}
$$

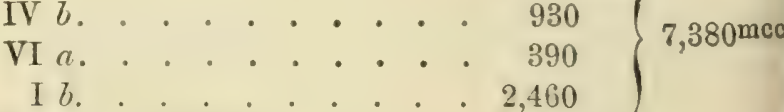

(Soit par an $369^{\mathrm{mec}}$ )

Appréciation du rolume à réaliser si la forêt était régulière et normale (en comparant aux forêts voisines) 
B.

Conversions de taillis en futaie.

Considérations générales. - J'ai expliqué dans le chapitre Ier quelles sont les considérations qui font un devoir à État de posséder des futaies et d'amener à ce régime la plus grande partie des taillis qu'il possède. Ces considérations sont applicables aux communes, à un certain degré, car elles forment une petite société près de laquelle l'État ne possède souvent aucune forêt, et dont les habitants ont pourtant besoin de bois de service. Bien sourent on a demandé : pourquoi ne pas traiter les bois des communes comme ceux des particuliers? pourquoi ce qui est bon et sage pour les fortunes privées ne serait-il ni bon ni sage pour le patrimoine des communes?

La réponse est facile: les futaies rapportent plus en volume que les taillis; mais le taux de la rente du capital engagé dans une exploitation en taillis est bien plus élevé que celui fourni par le régime de la futaie. Or, dans les communes francaises, il y a deux natures de parties prenantes, deux sortes d'individus qui ont droit aux produits du bois : les habitants d'une iart; la commune, ètre moral représentant la so:iélé communale, d'autre part. Pour les habjitints, la futstion du placement est fort indifférente; ce quil 
leur faut, c'est la plus grande production anruslle possible, et la futaie seule donne ce résultat. L'ètre moral est bien propriétaire du capital, mais il n'en a pas la libre disposition, il doit le transmettre intact aux générations futures; peu lui importe donc que ce capital soit gros ou petit, que la rente soit faible ou élevée; ce qu'il lui faut aussi, c'est la plus grande production annuelle possible en volume, parce qu'à ceite production correspond la plus grande somme d'argent dont il pourra disposer tous les ans. La société communale peut enfin aroir, de son còté, le plus grand intérèt à trouver dans les forèts de la commune les bois de service et d'industrie que les forèts particulières ne peuvent lui fournir ou que l'éloignement des forèts domaniales lui rend trop onéreux.

Ainsi, quand une commune possédera une forèt dont le capital sera constitué, ou à peu près constitué pour une exploitation en futaie, ce sera ce régime qui lui sera le plus avantageux, car il donnera la plus grrande satisfaction aux intérèts des habitants, à ceux de la caisse municipale et à ceux de la localité et des diverses industries qui y existent. Mais quand le capital d'exploitation ne sera pas constitué, il y aura souvent lieu à hésiter avant d'entreprendre une conversion en futaie. Ce ne sera que dans des circonstances exceptionnelles de communes fort riches, d'essences impropres au taillis et de forèts as- 
sez étendues pour se prèter à des combinaisons d'aménagement, que les conversions en futaie de taillis communaux auront la chance d'ètre menées à bonne fin. On conçoit, en effet, que quelles que soient les combinaisons employées, il y aura géréralement diminution dans le volume des produits, car il s'agit de passer d'un matériel de un à trente ans, par exemple, à un matériel de un à cent ving̣t ans, et une pareille transformation ne peut se faire sans du temps et sans une diminution certaine dans le rendement. Mais en faisant entrer dans ces combinaisons la création d'un ensemble de réserves sur les taillis, on ne diminue pas sensiblement la production des premières années, et ces futaies, dont l'accroissement est considérable, tendent à balancer par leur plus-value la diminution du volume. Aussi les méthodes de transformation de taillis simples en futaie se confondent avec celles des taillis composés, que je me bornerai à expliquer.

Je ferai une distinction entre les taillis composés qui ont toujours été traités régulièrement et ceux qui présentent des peuplements irréguliers provenant d'exploitations de différentes natures.

$$
\S 4 \mathrm{er} \text {. }
$$

Conversion de taillis sous futaie réguliers.

Il y a dans les taillis sous futaie deux natures de 
capital d'exploitation: celui du taillis répandu sur toute la forêt, suivant une échelle d'âges graduée de un à trente ans, par exemple, et celui de la futaie sur taillis qui est réparti sur chaque coupe suivant une échelle particulière dépendant du plan de balivage. On concoit, dès lors, que pour constituer un capital d'exploitation de un à cent vingt ans, on réalisera pendant ce temps, soit le capital du taillis qu présente une gradation d'âges sur toute l'étendue de la forêt, soit celui de la futaie qui est disséminé dans chaque coupe. L'un ou l'autre de ces matériels d'exploitation ne s'adaptant plus au mode de traitement projeté pour la forêt, pourra être exploité, utilisé et réparti sur tout le temps que devra durer la conversion. Là est la base des diverses méthodes employées : on cherche à créer un matériel d'exploitation de un à cent vingt, ans, et on consomme pendant ce temps un matériel existant qui peut être celuj des futaies sur taillis, et qui est assez souvent unt portion combinée de ce dernier et de celui de l'ancien taillis. La diminution de produits forcée, nécessaire, pendant la période d'attente, sera donc beaucoup attenuée par l'exploitation des réserves de l'ancien taillis et par les combinaisons qui peuvent se faire avec les éléments de l'ancienne exploitation. Il pourra même arriver, si le taillis est chargé dt nombreuses rẻserves, qu'il n'y ait que fort peu dt 
diminution pendant la révolution de conversion, mais cela dépendra des circonstances et surtout de la méthode employée : l'art de l'aménagiste consistera à rendre le sacrifice le moins lourd possible en le répartissant sur de longues années.

Ce sont ces considérations qui déterminent les caractères généraux des méthodes de conversion: Pour créer un capital d'exploitation formé par une échelle d'âge de un à cent vinğt ans, il faut, personne n'en doutera, rien moins que cent vingt ans! Mais si la forèt contient déjà des bois de un à trente ans, par exemple, dont on puisse se servir pour les faire entrer dans cette échelle d'àges, il faudra moins de temps, et en utilisant ce qui existe, on pourra abréger la durée de la rérolution de conversion: de là deux méthodes qui, par leur combinaison, donnent lieu à une troisième.

10 La méthode des coupes préparatoires, qui consiste à utiliser les peuplements existants dans le but de réduire la durẻe de la conversion ${ }^{4}$.

\section{$2^{\circ}$ La méthode du taillis temporaire, qui crée}

I Dans la première édition, cette méthode était désignée sous le nom de directe parce qu'on a pour but de créer directement la futaie arec le matériel d'exploitation du taillis, prétention inconciliable arec la rigueur des définitions, puisque le taillis est formé de rejets de souche, et la futaie de brins de semence. La dénomination de méthode directe s'appliquuerait également au procédé qui consiste à créer la futaie successivement par voie de régénération. Aussi, il nous a paru préférable de désigner chacune de ces méthodes par lo nom des coupes qui les caractérisent. 
AMENAGEMENT DES FUTAIES.

à neuf l'échelle des àges nécessaires à l'exploitation en futaie et emploie par conséquent pour la conversion une durée égale à celle de la révolution de futaie.

$3^{\circ}$ La méthode mixte, qui participe de la première et de la seconde, prépare les peuplements en vue d'une régénération naturelle, et complète son organisation en conservant l'exploitation en taillis temporairement sur certains points.

Celle-ci est naturellement la plus employée, car c'est elle qui convient le mieux aux circonstances habituelles de la régétation dans les forêts de France; c'est elle aussi qui se prête le mieux aux cas particuliers que peurent présenter les forèts irrégulières dont nous parlerons après aroir exposé chaque methode.

Il serait peut-être inutile, dans un livre élémentaire, d'indiquer le mécanisme des deux premières méthodes, si cette connaissance n'était nécessaire précisément pour comparer les résuliats et mettre en lumière les avantages de la dernière méthode.

Nous supposerons, pour préciser notre exposé, une forêt de $\mathbf{2 4 0}$ hectares traitée en taillis ì trente ans, qu'il s'aggit de transformer en futaie à exploiter à cent vingt ans 
I.

MÉTHODE DES COUPES PRÉPARATOIRES.

Toute la combinaison consiste à laisser vieillir les taillis et à se contenter pendant un certain temps des produits de nettoiements et d'éclaircies combinés avec l'extraction de certains arbres de futaie anciens et modernes: en laissant vieillir la forêt pendant vingt ou trente ans, par exemple, on obtiendra des bois âgés de vingt à cinquante ans ou de trente à soixante ans; on l'aménagera ensuite par un des procédés que nous avons indiqués pour les forêts dont le capital d'exploitation n'est pas constitué.

La durée de cette période d'attente est difficile à déterminer d'une manière exacte, car, en la prolongeant trop, on finirait par avoir un matériel trop âgé. L'expérience et la pratique sont à peu près les seuls guides pour cette détermination; tout ce que l'on peut dire, c'est que cette période doit être assez longue pour permettre l'assiette de l'aménagement avec une révolution de futaie, suivant un des modes que nous avons indiqués (page 116): on se contente généralement de faire la période transitoire égale à une révolution de taillis, trente ans dans notre exemple.

Le cadre de cet aménagement ultérieur est immédiatement assis sur le terrain; c'est sur lui et pour 
lui que se modèleront les peuplements. On créera, par exemple, quatre affectations de 60 hectares dans le but de correspondre un jour à 4 périodes de 30 ans. C'est donc un aménagement à l'application duquel il est sursis pendant une périnde préparatoire.

Pendant cette période transitoire, on parcourt la forêt arec des coupes qui ont été appelées préparatoires à la futaie ou éclaircies de conversion, dans lesquelles on coupe les brins dominés sous forme d'éclaircie, on exploite certains arbres et on raccorde des futaies avec les brins du taillis, de manière à créer des peuplements réguliers dans leur ensemble, sinon dans leurs éléments. Ces éclaircies se font par contenance et peurent parcourir la forêt deux fois dans la période transitoire, par conséquent par $1 / 15^{\circ}$ de surface. Leur caractère est déterminé par le numéro de l'affectation dans laquelle elles se trourent, c'est-à-dire que l'on s'appliquera à ménager dans la première affectation les vieux arbres pour concourir à la régénération; dans la dernière, les jeunes; dans les affectations intermédiaires, les sujels d'àge moyen.

A l'expiration de la période transitoire on entre dans le cadre définitif de l'aménagement en régénérant d'abord l'affectation $n^{0} 1$, ensuite celle $n^{\circ} 2$ et ainsi de suite; mais il faut bien remarquer que la forèt est, dans sa généralité, peuplée de rejets de 
souche sans assiette bien solide et sans avenir; il faudra le plus souvent raccourcir cette première rérolution, et si l'on reut exploiter plus tard à 120 ans, arec 4 périodes de 30 ans, on sera obligé de réduire dans une certaine mesure la durée de la première rérolution, de la fixer, par exemple, à quatre-vingts ans, à quatre périodes de vingt ans.

Ainsi le but principal qu'on avait en vue dans cette méthode, et qui était de profiter du matériel existant pour arriver plus tôt à une exploitation en futaie, est mal atteint; car, aprés une période d'attente de trente ans et une révolution transitoire de quatreringts ans, on aura mis en résumé cent dix ans pour opérer la conversion.

De plus, en composant les peuplements avec les rejets du taillis combinés avec certaines réserves, on expose la forêt à présenter des massifs mal composés, peu homogènes, qui n'ont de régulier que l'apparence et dont les chances de longévité sont fort compromises.

Aussi ce n'est que dans les taillis des régions montagneuses peuplées presque exclusivement de hèire et de chêne et dépourvies de bois blancs que cette méthode, jadis fort employée, pourrait avoir la chance de domner des résultats convenables.

Les coupes qu'elle emploie ont cependant un caractère dont l'importance ne saurait être méconnue 
et qui a été utilisé dans une méthode plus perfec. tionnée: c'est que les éclaircies de conversion préparent le terrain pour la régénération mieux que ne peut le faire la main de l'homme, en relevant le couvert, en combattant les rejets de souche qui sont le plus grand obstacle à la venue des brins de semences et en augmentant graduellement la quantité de terreau qui couvre le sol.

\section{II.}

MÉTHODE PAR LE TAILLIS TEMPORAIRE.

Le caractère de cette méthode est de ne pas compter sur le matériel qui existe pour constituer le capital d'exploitation en futaie et de l'affecter entièrement à fournir des produits pendant la conversion. L'échelle des âges de un à cent vingt ans se créera donc au fur et à mesure qu'on avancera, et la période de conversion sera forcément égale à la révolution adoptée, elle sera de cent vingt ans. C'est pour ce motif qu'on l'a appelée révolution de conversion.

La première opération sera donc de diviser la forêt en affectations pour avoir le cadre général, la forme suivant laquelle on modèlera les peuplements à créer. Quand une futaie est constituée, les affectations doivent satisfaire à une condition essentielle, qui est de correspondre à des périodes de régénération, c'est-à-dire au temps qui est nécessaire, selon 
le climat et la nature des essences, pour les régénérer complétement. Ici elles doivent satisfaire, non-seulement à cette condition, car c'est le cadre définitif de l'aménagement qui est ainsi tracé sur le terrain, mais encore à une autre, qui est de correspondre à une durée égale à une révolution de taillis, car, pendant qu'on régénérera la première affectation, on continuera sur les antres l'exploitation en taillis. Chaque affectation, exceptẻ celle en tour, sera ainsi une série temporaire de taillis dans laquelle ce mode d'exploitation durera plus ou moins longtemps, selon le numéro d'ordre de l'affectation.

Pour faciliter l'exposé de la méthode, nous supposerons que les 240 hectares aient été exploités en quatre séries de taillis de 60 hectares, à la révolution de trente ans: on prendra un plan d'exploitation de quatre affectations correspondant à des périodes de trente ans et formées chacune d'une des séries de taillis :

$$
\begin{aligned}
& \text { I. } 60 \text { hectares . . . } 1 \text { à } 30 \text { ans. } \\
& \text { II. } 60 \text { " • . . . } 1 \text { à } 30 \text { " } \\
& \text { III. } 60 \text { " . . . . } 1 \text { à } 30 \text { ” } \\
& \text { IV. } 60 \text { " . . . . } 1 \text { à } 30 \text { ” } \\
& 240 \text { hectares. }
\end{aligned}
$$

Le plan d'exploitation sera le suivant:

PREMIÈEE PERTIODE

(1860 ì 1889).

Ire affectation, régénération par voie de coupes d'abri 
par $1 / 15^{e}$ de surface par an, en revenant ensuite pour dégager les semis au moyen de coupes par contenance ou par volume.

II affectation, taillis à 30 ans, 2 hectares par an.

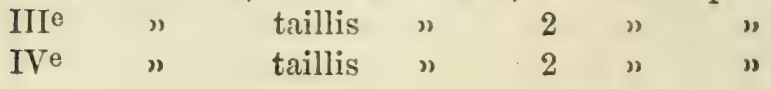

DEUXIÈME PÉRIODE

(1890 à 1919).

Ire affectation, nettoiements sur bois de 1 i 30 ans.

IIe " régénération, coupes d'abri.

III

IVe " taillis à 30 ans.

TROISIÈME PÉRIODE

(1920 aे 1949).

Ire affectation, éclaircies sur bois de 30 ì 60 ans.

IIe " nettoiements sur bois de 1 à 30 ans.

IIIe " régénération, coupes d'abri.

IVo " taillis à 30 ans.

QUATRIÈME PÉRIODE

(1950 à 1979).

Ire affectation, éclaircies sur bois de 60 à 90 ans.

II ${ }^{8}$ " éclaircies sur bois de 30 à 60 "

IIIe " nettoiements sur bois de 1 à 30 ans.

IVe " régénération par coupes d'abri.

Dans celte méthode, où les exploitations de taillis vont reculant et diminuant d'importance, au fur et à mesure que la futaie arance et se constitue, la diminution des produits pourra ètre assez faible. Pendant la première période, il pourra même y avoir augmentation, car tout le matériel du taillis de la 1 re affectation sera livré à l'exploitation. Ce ne sera, 
en réalité, qu’à la seconde période, oủ la première affectation ne donnera que des nettoiements peu importants, que la diminution se fera sentir; mais si l'on a soin de modifier le plan de balivage en prévision de cette diminution, et d'augmenter les réserves sur les parties qui doivent ètre traitées en taillis temporairement, on arrivera à augmenter les chances de la régénération naturelle et à équilibrer la production en la maintenant pendant toute la durée de la révolution à peu près, sinon au volume, du moins à la valeur de celle de l'ancien taillis.

Comme il n'y a aucune utilité à créer dans chaque affectation une série temporaire de taillis, on aug̃mentera les chances de réussite des coupes d'ałri de l'affectation en tour (dans laquelle les semis ont toujours à lutter arec les rejets de souche) en modifiant la composition des anciennes séries de taillis de manière à grouper dans la première affectation les vieux taillis qui se prètent mieux aux coupes d'abri. On réunit ainsi temporairenent les aflectations ou le taillis cloit se continuer en une seule suite de coupes dont l'importance diminue à chaque période et qui liwre ainsi, à chaque changoment d"affectation, les plus vieux taillis aux coupes d'abri.

Si la forèt a été exploitée en me scule sirie de taillis à trente ans, à raison de 8 hectares par an, la formation des affectations est toute naturelle. 
I. 60 hectares, taillis de 24 ì 30 ans.

II. $60 \quad "$
III. $60 \quad$ "
IV. 60
240 hectares.

Si, au contraire, la forêt a été exploitẻe en plusieurs séries, on cherche à grouper les âges de manière à constituer une première affectation avec les taillis les plus vieux et une suite de coupes se prêtant, avec des écarts tolérałles dans les âges, à une exploitation en taillis en une seule série.

Le plan d'exploitation se résume ainsi qu'il suit : 
要

$\infty$

$\therefore 2$

$2=$

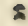

0

Hen

o

芦

..

¿

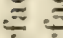

$\equiv$

$\stackrel{2}{=}$

$\ddot{0}$

를

$\stackrel{\circ}{*}$

$\therefore \stackrel{\circ}{5}$

-

के

L

๘

تै

ब.

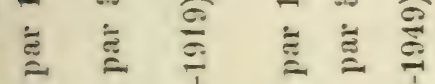

商

\&

离:

.

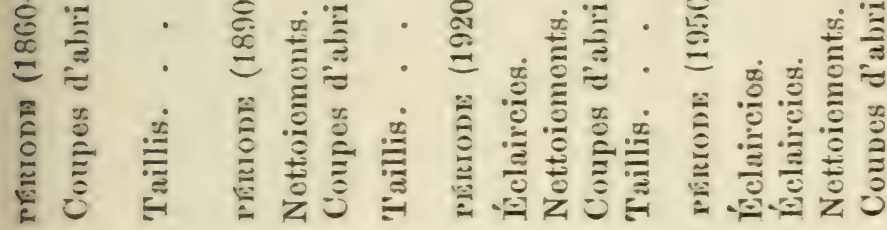

西

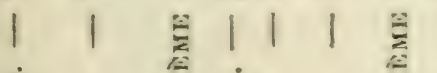

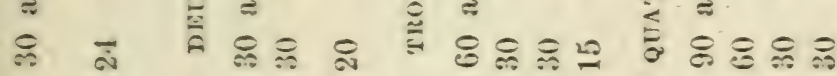

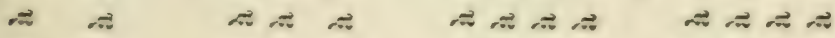

茫 -

密

:
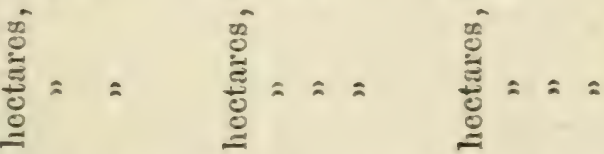

$\& \approx$

-

$\therefore \therefore 8:$

요.

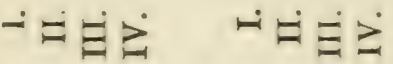

Parmi ces deux modes d'application d'une mème méthode, c'est le second qui atténue le plus l'incon- 
vénient caractéristique du procédé de conversion. Cet inconrénient vient de ce que les taillis fournissent toujours des rejets de souche qui tendent à ètouffer les semis, et si les arbres de futaie ne sont pas assez nombreux pour tuer les cépées dans le sous-bois, les coupes d'abri demanderont toujours des soins et des dépenses considérables'.

\section{III.}

MÉTHODE MIXTE.

Dans la méthode précédente on a l'arantage de pouvoir introduire les essences les plus propres à la futaie, le chène, les résineux, par exemple, et de constituer des massifs réguliers et homogènes ; mais cetle méthode a pour inconvénient:

10 D'ètre coûteuse, car les jeunes peuplements se forment à chaque période et dans chaque affectation par voie le plus souvent artificielle, au milieu de rejets dont il faut les défendre;

$2^{\circ}$ De rejeter sur l'avenir et à une époque dont on ne peut actuellement apprécier les exigences, le moment probable de la diminution des produits, ce qui peut compromettre un jour le succès de la conversion;

IIl faut constater aussi arec Mr. Sée (Re\%. for., 1867, p. 263) que, pour donner des rieux taillis aux coupes d'abri, on est conduit à commencer les coupes des taillis, temporairement conservés, à 24 et même à 15 ans, ce qui fournira, bien dificilement dans les premières années, des baliveaur convenables. 
$3^{\text {e }}$ De continuer le taillis pendant $1,2,3$ et souvent 4 périodes; or il arrive souvent que les taillis sont déjà fort mauvais et que le terrain épuisé se refuse à la continuation de ce régime.

Il n'y a rien d'absolu dans les applications d'aucune science; cela est surtout vrai en matière forestière; la première méthode, malgré ses inconvénients, s'est appliquée fort bien à des taillis de hêtre et dans des circonstances particulières; la seconde convient à la transformation de taillis simples ou de taillis composés peuplés d'essences diver'ses et de bois blancs impropres à former des massif's de futaie. Les inconvénients et les avantages de l'une et de l'autre ont conduit à adopter un système mixte qui emprunte les coupes préparatoires à la première méthode, les coupes d'abri et l'assiette définitive de l'aménagement à la seconde, et qui est, par conséquent, applicable au plus grand nombre des cas si nombreux et si variables que présentent les forêts.

Conservons toujours le même exemple et supposons qu'on veuille maintenir le taillis à trente ans pour ne pas répéter trop souvent des exploilations dort la brièveté de la révolution a pour effet de détruire les bonnes essences et de les remplacer par des bois blancs. Le plan général qui doit, d’après ly principe que nous avons si souvent posé, ètre assis 
sur le terrain, sera de quatre affectations correspondant à des périodes de trente ans.

I a méthode mixte consiste à faire, cr meme temps que les coupes d'abri sur l'affectation en tour, des coupes préparatoires sur l'affectation qui suit, et des coupes de taillis sur les autres, et ainsi de suite. Le régime du taillis est abandonné à chaque période sur une affectation, non plus pour la régénérer, mais pour préparer son peuplement à fournir à la période suivante les éléments d'une régénération naturelle qui n'existent pas dans l'état actuel, et pour diminuer la dépense des repeuplements artificiels. Les futaies de l'ancien taillis conservées dans les coupes préparatoires apportent à la régénération des éléments utiles; mais il sera nécessaire de compenser sur un autre point la diminution de production qui en résultera. Dans ce but, la série de taillis qui précède immédiatement la série des coupes préparatoires et qui ne doit durer qu'une révolution sera exploitée arec un balivage spécial qui consistera à abattre principalement les trop vieilles réserves, en conservant surtout des modernes et des baliveaux, ceux-ci en très-grand nombre, et de manière à faciliter pour la période suivante les coupes préparatoires. 
Le plan d'exploitation sera le suivant :

1'e PÉRIOdE (1860-1889).

Ire affectation. 60 hectares. Coupes d'abri par $1 / 10$ de surface, 3 fois dans la période pour faciliter les exigences de semis. 6 hectares par an.

IIe affectation. 60 hectares. Coupes préparatoires par $1 / 15^{\circ}$ de surface. 2 fois dans la période pour augmenter les produits et assurer le succès des coupes. 4 hectares par an.

III affectation. 60 hectares. Taillis, en exploitant les trop vieilles futaies et réservant un très-grand nombre de balireaux et de modernes choisis parmi les essences dures. $1 / 30^{e}$ de surface. 2 hectares par an.

IVe affectation. 60 hectares. Taillis avec balivage ordinaire. $1 / 30^{\mathrm{e}}$ par an. 2 hectares.

\section{$2^{e}$ PÉriode (1890-1919).}

I. Nettoiement de peu de valeur sur bois de 1 à 30 ans. 2 hectares par an.

II. Coupes de régénération dans un massif déjà composé de forts brins et de futaies et dans un sol déjà approprié par le courert. 6 hectares par an. -3 fois.

III. Coupes préparatoires dans un clair perchis de 30 à 60 ans, mélangé de taillis. 4 hectares par an. -2 fois. IV. Taillis arec réserve de nombreux balireaux et exploitation des vieilles futaies.

\section{$3^{\text {e }}$ PÉRIOde (1920-1949).}

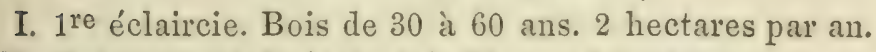

II. Nettoiement. Bois de 1 à 30 ans. 2 hectares.

III. Régénération. Jeune futaie claire de 60 à 90 ans mélangée de taillis. 6 hect. par an, ou coupes par rolume. IV. Coupes préparatoires, perchi‘ mélangé de taillis de 30 à 60 ans. 4 hectares par an.

4e PÉRIOdE (1950-1979).

I. $2^{2}$ éclaircie. Bois de 60 ì 90 ans. 2 hectares. 


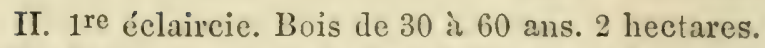
III. Nettoiement. Bois de 1 it 30 ans. 2 hectares.

IV. Régénération. Jeune rutaie de 60 à 90 ans. 6 hectares par an, ou coupes par volume.

Malgré son apparence compliquée, ce plan d'ex-ploitation est très-simple et d'une marche très-facile; i) n'exige que des conditions de peuplement qu'il sera toujours aisé de réaliser, surtout si la forèt à transformer a été exploitée en plusieur's séries de taillis: ces conditions sont d'avoir pour certaines affectations, et surtout pour la troisième, une échelle d'àges de un à trente ans au moins, de manière à donner un bon choix de baliveaux âgés chaque année, au minimum, de trente ans.

On colloquera, autant que possible, dans la première affectation les parties qui présentent des semis naturels qu'on rencontre très-sourent dans les taillis surchargés de réserves, et on réunira dans cette affectation tout ce qui peut contribuer à assurer le succès des coupes de régénération.

Si cette condition ne se trouve pas remplie, la méthode n'en est pas moins applicable, car il suffit, au lieu de régénérer immédiatement la première afiectation, de faire précéder cette régénération d'une période dans laquelle on prépare les peuplements. On débuterait ainsi en faisant des coupes préparatoires dans la première affectation, ce qui impliquerait une période d'attente avant la révolution de régé- 
nération et ce qui allongerait d'autant la durée de la transformation de la forèt. Ainsi conçue, la méthode est générale et se plie aux cas les plus divers que peurent présenter les forêts de taillis; mais parfois il n'est nul besoin de cette période, et on trouve la préparation toute faite dans certains cantons surchargés de réserves dues à une sage épargne.

Si le plan d'exploitation est bien suivi, on sera à peu près certain de n'avoir qu'une très-légère diminution, sinon dans le volume, du moins dans la valeur des produits de l'ancienne forêt, et d'arriver ainsi, dans un temps fort long, sans doute, mais d'une manière efficace et sans notables sacrifices, à une augmentation très-considérable du capital et de la fortune du propriétaire.

Cette méthode mixte est d'une application beaucoup plus générale et beaucoup plus certaine que les deux premières; mais elle suppose toujours une condition essentielle pour en recueillir tous les bénéfices : c'est que la généralité de la forêt soit peuplée d'essences dures et propres à la futaie, dans une proportion suffisante pour constituer ultérieurement un massif de régénération. - Il suffit pour cela

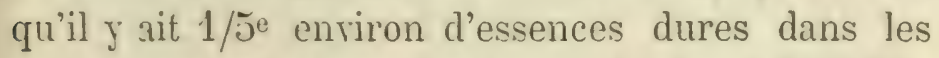
taillis. - Dans le cas contraire, les repeuplements artificiels seront toujours nécessaires.

Le registre de contrôle est très-facile à tenir et 
s'adapte très-bien à la formule générale que nous avons indiquée, si l'on a soin de tenir un compte

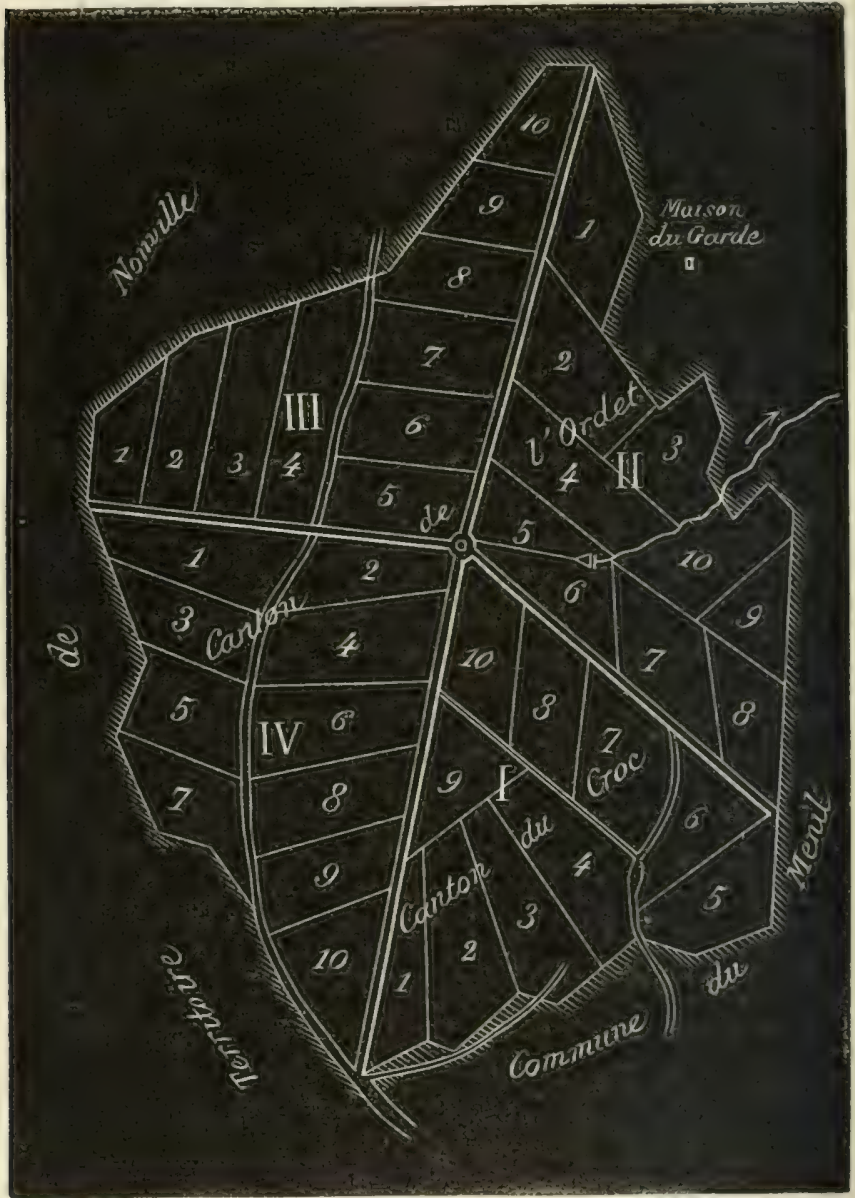

Fig. 8. - Conversion de taillis en futale.

spécial pour chaque nature d'exploitation: coupes 
d'abri, coupes préparatoires, coupes de taillis, ainsi que nous en arons donné le conseil p. 72.

On peut diviser les coupes sur le terrain, ce qui facilite singulièrement l'exécution de la conversion et surtout la rérification de la manière dont elle est conduite. On peut aussi, dans le but de diminuer la dépense, se borner à diviser chaque affectation en dix dirisions qui seront cotées:

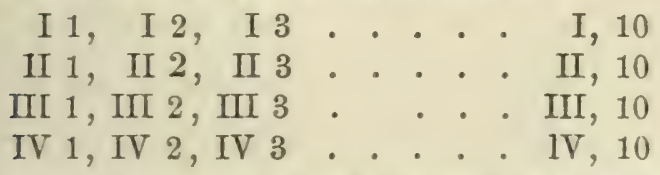

ct qui serriront: une pour la coupe d'al)ri, $2 / 3$ pour la coupe préparatoire, $1 / 3$ pour la coupe de taillis de chaque année.

De la Réserve. - Ce partage en dix divisions, outre son mérite d'économie, a encore l'arantage de faciliter l'établissement d'une réserve de préroyance qui, dans les forêts particulières et communales, a une très-grande importance. Les communes tiennent souvent, en effet, à conserver des ressources pour les besoins imprérus de la caisse communale, à laquelle la loi affecte d'ailleurs le quart de leur bois, et l'omission de cette réserve dans une combinaison de conversion pourrait, parfois, en compromettre le succès.

Dans les forêts communales, la réserve doit porter sur le quart des produits de la forêt; mais les coupes 
préparatoires n'étant que de simples éclaircies peu productives, on peut ne pas en tenir compte et ne faire porter la réserve, comme dans les futaies, que sur les coupes principales qui sont ici les coupes de taillis. Jusqu'au moment où les coupes principales se feront par volume et oủ la réserve sera prélevée sur le matériel à exploiter, on la constitue par une distraction d'un quart sur la contenance des coupes de Ire, III e et IVe affectation.

Ainsi, dans la première, on destine trois divisions à fournir quatre coupons de 4 hectares 50 , dont les trois premiers représentent la coupe annuelle, distraction faite de la réserve, et le quatrième, la coupe de réserve, et on les exploite en trois ans.

Dans la troisième et dans la quatrième, on destine une division à fournir quatre coupons de 1 hectare 50, qu'on exploite en trois ans, les trois premiers pour l'ordinaire, le dernier pour la réserve.

Le registre du contrôle permettra toujours de suivre facilement la situation de la réserve airısi prélevée sur la contenance. Tous les trois ans, on subdivisera, par un arpentage, en quatre parties égales, pour former quatre coupons, trois divisions réunies de la première affectation et une seule division de chacune des troisième et quatrième affectations. Les exploitations qui se font dans une conversion n'étant que temporaires, il n'y aura point d'in- 
conrénient à anticiper ou retarder quelque peu les coupes de la réserve pour les faire correspondre aux hesoins extraordinaires, et la réserve sera ainsi toujuurs à la disposition du propriétaire d'une manière Jien suffisante.

\section{§2.}

Conversion des taillis sous futuie irréguliers.

Les opérations de conversion ne se présentent pas toujours dans cet état de régularité théorique que nous arons pris pour exemple, parce qu'il était important de ne point compliquer l'exposé de la méthode par des circonstances particulières. Les taillis à convertir peurent être très-irréguliers : on a pu y commencer déjà des opérations de conversion; on a pu aussi y introduire des essences résineuses qui ne se prètent plus à l'exploitation en taillis; ces opérations ont pu être faites sur certains points de la forèt, tandis que, sur d'autres, des exploitations diverses ont eu lieu selon la nature des peuplements. Trés-nombreux et très-rariés sont les cas qui peuvent se présenter, mais aussi très-rariables et trèsfëcondes sont les combinaisons de l'aménagement.

L'agent forestier qui sera chargé de dresser un plan d'exploitation pour ces forèts irrégulières se basera sur les principes qui viennent dè̀tre exposés en combinant les opérations culturales que com- 
portent les peuplements avec le carlre général de l'aménagement qui devra toujours être assis sur le terrain. Des jeunes bois pourront ètre, par exemple, classés en quatrième affectation, au lieu des coupes de taillis dont nous avons parlé; des semis déji réussis pourront de même être classés dans la première affectation au lieu des coupes de régénération. On empruntera aux aménagements de futaies et aux trois méthodes de conversion des combinaisons pour dresser un plan d'exploitation convenable. Mais ces aménagements sont généralement difficiles, et il faut, chez celui qui en est chargé, outre un jugement net et une certaine science, une grande pratique et une longue expérience des forêts.

Exemple. - G'est dans ces aménagements qu'il pourra y avoir lieu à des périodes transitoires dont la durée ne sera pas égale à celle des périodes de régénération. Voici, par exemple, une forêt communale de 240 hectares, dont le quart de réserve, de 60 hectares, présente un bon perchis de hêtre de soixante-dix ans, entrecoupé d'arbres plus âgés, et dont les 180 autres hectares ont été exploités en taillis à trente ans, à raison de 6 hectares par an. Par suite d'essais de transformation en futaie, les dix premières coupes présentent un jeune perchis assez régulier et en bon état, de trente à quarante ans. Les vingt autres coupes, c'est-à-dire 120 hectares, offrent des taillis 
irréguliers et en maurais état : on veut transformer en futaie à la révolution de cent vingt ans.

On divisera la forèt en six affectations de 40 hectares environ, qui, plus tard, correspondront à des périodes de vingt ans; on constituera la première par 40 hectares de la réserve, âgés de soixante-dix ans; la deuxième, par les 20 hectares restant du mème àge et 18 hectares formant les trois coupes les plus àgées des dix qui ont été éclaircies et qui ont quarante ans; la troisième, par les sept autres coupes de même nature qui ont trente ans, et qui forment 42 hectares; les trois dernieres, par les taillis les plus mauvais formant 120 hectares. Pendant une période transitoire de trente ans, on exploitera, en taillis à trente ans, ces trois dernières affectations, et la production sera fournie pendant cette période:

10 Par des coupes d'extraction de certains arbres faites par trentieme du rolume actuel sur les trois premières affectations;

2. Par une coupe de taillis de 4 hectares sur les 120 hectares qui forment les trois dernières affectations, en assurant, à chaque coupe, la régénération par des semis artificiels.

Pendant cette période, le quart de réserve sera assuré par un prélèrement du 1/3 sur le rolıme des arbres à extraire dans les trois premières affectations, de manière à ne pas porter sur les coupes de taillis. 
$\Lambda$ la fin de cette période transitoire de trente ans, la forèt sera en état d'ètre exploitée suivant le cadre de l'aménagement définitif, car on aura le groupement suivant :

\begin{tabular}{|c|c|c|c|c|c|c|}
\hline 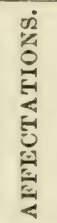 & 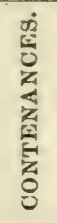 & 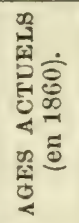 & 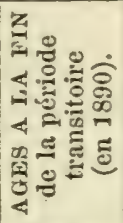 & $\begin{array}{c}\text { PÉRIODE } \\
\text { de } \\
\text { l'exploi- } \\
\text { tation. }\end{array}$ & 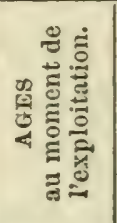 & $\begin{array}{c}\text { OBSERVA- } \\
\text { TIONS. }\end{array}$ \\
\hline I & 40 & 70 & 100 & $1890-1909$ & 110 & \\
\hline \multirow{2}{*}{ II } & 20 & 70 & 100 & 104 & 130 & \\
\hline & 18 & 40 & 70 & & 100 & \\
\hline III & 42 & 30 & 60 & 1930-1949 & 110 & \\
\hline IV & 40 & 30 & 20-30 & 1950-1969 & $90-100$ & \\
\hline V & 40 & à & $10-20$ & 1970-1989 & $100-110$ & \\
\hline VI & 40 & 1 an & $1-10$ & 1990-2009 & $110-120$ & \\
\hline
\end{tabular}

\section{CHAPITRE II.}

DES FUTALES TRAITÉES SUIVANT LA MÉTHODE DU JARDINAGE.

Généralitès. - Je dois dire quelques mots d'un autre mode d'exploitation des futaies qui a eu longtemps des admirateurs passionnés et ensuite des critiques non moins ardents, mais qui a eu, du moins, le mérite incontesté de nous avoir transmis dans un assez bon état les sapinières et les anciennes 
forèts des pays de montagnes; je veux parler du Jardinage.

Il consiste $\dot{a}$ enlever cà et là les arbres les plus vieux, les bois dépérissants ou d'autres en bon état de croissance qui sont parvenus aux dimensions que le propriétaire s'est fixé pour les livrer à la consommation; il est constitué par un mélange confus de bois de tous âges et de toutes dimensions.

Les anciens forestiers, qui n'avaient pas des idées très-nettes sur la constitution du matériel d'exploitation dans les forèts, navaient imaginé aucun autre moyen pour empècher d'entamer ce matériel que de fixer d'une manière très-approximative le nombre d'arbres à exploiter tous les ans. Le forestier français Dralet se contentait de prescrire la coupe de trois à cinq arbres au plus par hectare et d'étendre, autant que possible, le jardinage sur toute la forèt. Quand Dralet parle ainsi de trois à cinq arbres par hectare, il comprend évidemment dans ce nombre, non-seulement quelques arbres exploitables, mais en outre des arbres morts ou dépérissants de toute grosseur'; autrement il arriverait à dépasser notablement la production qu'il est permis d'espérer des futaies les mieux entretenues.

Nous avons vu que dans une forèt traitée en fulaie pleine que l'on veut exploiter à l'àge de 120 ans, 1.M. Bagrнeris y Hanueb de Sylviculture, p. 109. 
le matériel d'exploitation est une suite de peuplements âgés de un à cent vingt ans, échelonnée sur chaque élément de contenance (sur chaque hectare par exemple, si la forêt a 120 hectares), desorte que le premier hectare présente des bois de un an, et le dernier, des bois de cent vingt ans. Dans le jardinage, au contraire, chaque hectare porte non-seulement l'échelle complète des âges, depuis le brin qui vient de naître jusqu'à l'arbre de cent vingt ans, mais encore l'échelle des volumes que comporte le peuplement de chaque âge, depuis le fourré, le gaulis et le perchis, jusqu'aux deux ou trois arbres qui sont exploitables: chaque hectare forme ainsi à lui seul une série complète d'exploitation.

Il faut en déduireque, si le nombre d'arbres à exploiter annuellement a été convenablement réglé et si les coupes ont été constamment conformes à cette base, le matériel d'exploitation d'une forèt traitée en jardinage doit être aussi considérable en volume que le capital d'exploitation d'une forèt traitée en futaie pleine. Que ce matériel soit régulièrement distribué par hectares et par âges ou que tous les âges soient mélangés sur chaque hectare, il n'y en aura pas moins, si l'échelle est complète et sans interruption, parité absolue entre le volume des bois qui forment le capital d'exploitation des futaies dans l'un ou l'autre mode de traitement. Cette conception n'est que 
théorique; elle est analogue avec l'idée que l'on a dû se faire de la forèt normale pour la futaie pleine; elle n'est pas moins nécessaire que cette dernière pour éclairer les comparaisons et justifier la pratique des exploitations.

On conçoit que, dans une forèt dont le capital d'exploitation est théoriquement ainsi disposé, il faudrait, à la rigueur, aller chercher tous les ans, dans chaque hectare, les deux ou trois arbres qui ont atteint l'àge de cent vingt ans et les quelques brins dépérissants qui complètent le nombre fixé. Or cela n'était pas possible, et l'on se contentait, dans les exploitations, de couper tous les ans le nombre de pieds d'arbres admis, quatre, par exemple, multiplié par le nombre des hectares, soit quatre cent quatre-vingts arbres, en parcourant la plus grande étendue de terrain possible. L'année suirante, on jardinait, en suivant, la même quantité l'arbres, de manière à parcourir ainsi toute la forèt en très-peu d'années, cinq à dix ans, selon l'imporance de son étendue.

On conçoit aussi que, rien ne réglant l'étendue des norceaux de forêt à jardiner par an, le caprice et l'arjitraire présidaient seuls à l'exploitation, et il n'ètait jas possible de se rendre compte du matériel de la orêt.

Tel est donc l'état actuel des forèts jardinees: un 
mélange confus de bois de tous àges et de toutes dimensions, sans aucun moyen de déterminer l'importance et la composition de ce mélange.

Quant on veut mettre de l'ordre dans ces exploitations, la première mesure qui se présente à l'esprit est de régler par des contenances bien définies l'étendue des exploitations qui se faisaient tous les ans un peu au hasard. On partage donc la forêt en un certain nombre de divisions, en 10, par exemple, de 12 hec. chacune, que l'on cote par des numéros de 1 à 10 , et sur lesquelles on jardine, chaque année, les quatre cent quatre-vingts arbres réglés par l'usage ancien, en ne revenant que chaque dix ans sur chacune. Ces divisions sont formées de contenances égales ${ }^{1}$, autant que possible, et sont déterminées par des circonstances de configuration, d'exposition ou de situation. Le but de ce partage en divisions égales est de circonscrire les exploitations dans un espace déterminé, de faciliter ainsi le succès des ventes et d'assurer la surveillance sur les points oi se fait la coupe: c'est la première mesure à prendre

1 La contenance de ces divisions doit varier dans des limites telle que le succès de la vente des coupes ne soit pas compromis par une trop grande étendue de terrain à parcourir. - Si la forêt est très-considérable, on y crée plusieurs séries de jardinage ayant chacune un nombre déterminé de divisions. Ces séries permettent, dans les pays de montagnes, d'adoptel différentes bases de jardinage conformes aux exigences locales de lạ ré. . étation, cal celles-ci sont sourent très-diverses suivant les situations et les zones d'altítude. 
our exploiter une forêt en jardinant. Son effet est le modifier un peu la manière dont le capital d'exploiation est constitué: au lieu d'avoir sur chaque hecare de la forêt l'èchelle complète des âges et des euplements de l'exploitation à cent virigt ans, on end à avoir dans chacune des dix divisions une chelle d'àges et de peuplements complète; on vise avoir ainsi dix petites séries d'exploitation à coupe écennale, au lieu des cent vingt séries théoriques à oupe annuelle de l'ancien jardinage. On a toujours ien la même confusion d'àges et de bois, mais cette onfusion tend à se circonscrire dans chaque division $t$ à n'en pas dépasser les limites.

De la Possibilité. - Les divisions créées, il imorte de déterminer le chiffre de la coupe annuelle, 'est-à-dire le volume ou le nombre d'arbres à exloiter tous les ans, pour ne pas être exposé à entarer le capital. On conçoit que la contenance ne peut tre une mesure suffisante pour régler la quotité de exploitation, car cette contenance n'a pour but que e circonscrire les coupes de chaque année sur un oint determiné.

On s'est beaucoup ingénié à trouver une solution ce problème de la possibilité des forèts jardinées.

Les uns, partant de ce principe que dans une fuie jardinée le capital d'exploitation est le mème xe celui d'une futaie pleine exploitée au mème àge, 
ont roulu déterminer l'accroissement en adoptant simplement la production des futaies pleines de même essence, situées dans des conditions analogues et dont ils diminuaient un peu le chiffre pour rester dans les limites d'une sage modération. Si une futaie pleine, située dans desconditions analogues, rapportait 4 mètres cubes à l'hectare, ils adoptaient 3 mètres cubes 500 , ou 3 mètres cubes 750 pour la futaie jardinée, et le produit de ce chiffre multiplié par la contenance de la forêt était considéré comme donnant une limite de possibilité suffisante.

Il est bien certain que, théoriquement, le capital d'exploitation d'une futaie à cent vingt ans est tout aussi considérable dans celle qui est traitée en jardinage que dans celle qui est exploitée suivant la mé. thode de futaie pleine (v. p. 158). Mais dans l'état actuel des futaies jardinées, où le caprice et l'arbitraire étaient la seule règle des exploitations, il est tout à fait impossible de constater s'il y a des lacunes dans les âges et dans les volumes correspondants. Admettre une comparaison arec une futaie pleine pour déterminer la production, c'est admettre que le matériel d'exploitation est constitué en volume, en âges et en peuplements en vue de l'exploitabilité que l'on a choisie; or rien ne l'indique et il n'existe nul moyen de s'en assurer.

Les autres, essayant de déterminer en volume le 
matériel d'exploitation, ont prétendu que ce matériel, étant égal à celui de la futaie pleine, devait être, par conséquent, sur chaque hectare, égal à la moitié du volume de l'hectare de futaie pleine exploitable. Si le volume de celui-ci est de $\mathbf{4 0 0}$ mètres cubes à cent vingt ans, le capital d'exploitation sera le produit de la contenance par 200 mètres cubes, c'est-à-dire que l'on devra toujours avoir 200 mètres cubes sur chacun des hectares de la forêt jardinée. Celle--ci devant être exploitée à cent vingt ans, le matériel actuel devra rester en moyenne soixante ans sur pied, et la division du matériel normal, 200 mètres cubes, par 60 donnera la possibilité. On devait donc comper le volume de tous les arbres de toute catégorie existant dans la forêt; s'il était plus considérable que le matériel d'exploitation admis pour le jardinage, l'excédant formait des coupes extraordinaires i exploiter dans un certain intervalle de temps; s'il tait plus faible, on diminuait un peu les coupes innuelles jusqu'à l'époque fixée pour une révision lu matériel.

Ceux qui ont voulu déterminer ainsi le matériel l'exploitation des futaies jardinées et l'évaluer en nètres cubes sont tombés dans une double erreur : Jour établir le matériel de 200 mètres cubes de 'exemple précédent, ils ont d'abord admis que, dans es futaies pleines, le volume des bois de un à cent 
vingt ans qui forment le matériel d'exploitation sui ane ligne droite ascendante de 1 ì 400 mètres cubes c'est supposer connue la loi de la régétation, qui es fort loin d'être dans le domaine de nos connaissances actuelles et qui, en tout cas, n'est pas une lignt droite, mais une courbe plus ou moins compliquẻe.

A cette première erreur, ils en ont ajouté une au. tre, c'est que le matériel d'exploitation n'est pas formé seulement par un volume, mais par des catégories d'âgres réparties de telle sorte qu'aucune nı manque et que toutes existent en proportion conve. nable. Or il est impossible de déterminer, dans lt mélange confus des forèts jardinées, si cette conditior existe, et il pourrait très-bien se faire que le volum admis pour le chiffre du matériel normal d'exploita. tion ne fût représenté que par des vieux bois or par des bois d'àges non convenablement gradués On n'aura donc jamais, en procédant ainsi, qu'ur chiffre fictif de possibilité, incapable de servir dt base à l'exploitation raisonnée d'une forêt.

Pour régler la possibilité d'une forèt jardinée, or n'a donc rien trouvé et l'on ne trouvera, très-pro. bablement, jamais rien de satisfaisant; on peut mèmt dire que la détermination exacte de la possibilité de: forêts jardinẻes est un problème incompatible aver ce mode d'exploitation. Il est évident, en effet, qu dans un mélange aussi confus d'àges et de peuple 
ments que présente le jardinage, le matériel sera toujours loin d'ètre disposé comme la théorie l'indique et que les divisions créées pour le régulariser seront loin d'ètre semblables entre elles sous le rapport du rolume qu'elles renferment et de la suite d'àges et de peuplements qu'elles contiennent. Le volume qu'il s'agit de déterminer ne sera donc jamais une possibilité dans le sens précis de ce mot; il ne sera qu'une limite, un simple renseignement donnant un chiffre qu'il ne faudra point dépasser. L'état des peuplements sera toujours le véritable guide du forestier qui aura soin de ne créer aucune clairière et de ne jamais enlever un arbre rif que lorsque des voisins plus jeunes pourront les remplacer.

Il ne faut donc pas chercher de base ni même de renseignement certain sur la possibilité de ces forèts dans lesquelles le mode de traitement n'est qu'une affaire d'expérience et de pratique. Nous rerrons plus loin cependant que les motifs qui font encore conserver le jardinage sont peu nombreux, mais qu'on rencontre assez fréquemment les circonstances où ils se présentent.

D'un autre còté, il est impossible, dans les grandes gestions de forèt, d'aroir des rénisseurs expérimentés partout, et il faut aroir un moyen de contròle et de surveillance.

Le seul moyen que l'on puisse imaginer est de fixer 
la dimension des arbres, - le diamètre ou la circonférence, - au-dessous de laquelle il sera défendu de couper aucun arbre vif. Ceux qui feront la coupe ne pourront exploiter que les arbres qui auront atteint ou dépassé cette dimension et n'y joindre que ceux qui sont morts ou qui, ayant une dimension inférieure, sont complétement dépérissants.

Il faut bien remarquer, du reste, que cette détermination d'un diamètre minimum est bien plutòt la fixation d'un terme d'exploitabilité qu'une donnée quelconque sur la possibilité.

Fixer le diamètre auquel on veut exploiter les arbres d'une forèt, c'est, en effet, déter'miner le degré d'utilité des produits qu'on veut retirer de cette forèt, c'est-à-dire l'àge auquel on veut couper ces arbres. On ne serait renseigné sur la possibilité que si l'on pouvait déterminer le nombre des arbres de ce diamètre qu'il faudra exploiter par an, et le nombre des tiges dépérissantes qu'il faudrait y joindre avec leur volume correspondant; or cela est et sera toujours irréalisable par le double motif:

1. Que l'ignorance des lois de la végétation nous empèche de déterminer le nombre des tiges que présentent par unité de surface les peuplements forostiers à chacun des àges de leur vie;

2. Qu'il n'existe nul moyen de s'assurer, dans des peuplements aussi mélangés et aussi confus que ceux 
résultant du jardinage, si le matériel d'exploitation est constitué ou surabondant ou se présente avec des lacunes dans ses catégories d'âges.

Si je me suis étendu sur cette question de la possibilité des forêts jardinés, c'est pour bien montrer l'inanité de recherches trop sourent tentées, et l'infériorité de ce mode de traitement sur celui de 'a futaie pleine.

Aménagement. - Cette impossibilité oú l'on est de déterminer, même à titre de renseignement, un chiffre quelconque de possibilité, n'empêche pas de soumettre ces forêts à un mode d'aménagement compatible avec leur état. On procédera de la manière suivante: on partagera, d'après les bases que nous avons indiquées, la forêt en divisions de contenances à peu près égales, correspondant à chacune des années de la période de iardinage (celle-ci doit être courte), en prenant pour ces divisions autant que possible des parties semblables par la configuration, l'exposition ou des particularités permanentes. On fera dans chaque division le comptage et le cubage des arbres qu'il convient d'enlever dans la période de jardinage, soit parce qu'ils sont dépérissants, soit parce qu'ils dépassent la dimension voulue. On divisera le volume total par le nombre d'années de la période de jardinage, et le quotient sura le chiffre de la coupe annuelle moyenne pour cette période. On 
prendra chaque coupe en répartissant les divisions proportionnellement aux rolumes qu'on y a trouvés, en s'attachant à former chaque contingent annuel d'une parcelle entière et, à defaut, soit d'une partie aliquote de parcelle, soit de plusieurs parcelles réunics, de telle sorte que les coupes annuelles soient à peu près égales, c'est-à-dire diffèrent le moins possible du volume annuel moyen.

Ceite dernière condition ne sera jamais que fort secondaire, et en faisant au début de la période de jardinage les comptages et les cubages qui fixent l'importance de cette coupe annuelle moyenne, les forestiers ne derront jamais oublier que la modération dans les exploitations est la meilleure règle du jardinage.

Au bout de la période, on recommencera le même comptağe et la mème division du matériel à exploiter dans la période suivante. Le rapport ne sera, sans doute, pas soutenu d'une période à l'autre; mais cette condition nous parait incompatible avec le jardinagre dans les forèts à aménager, telles que nous les arons aujourd'hui. N'avons-nous pas vu d'ailleurs, dans les futaies pleines dont le matériel est surabondant ou insuffisant, cette condition du rapport soutemu faire défaut d'une période à l'autre et se trouver mème inronciliable, dans bien des cas, avec les règles d'une exploitation bien entendue? 
Tout ce que l'on peut dire, c'est que si, comme nous l'avons conseillé, on a créé dans la forêt des séries correspondant à des zones de différences bien tranchées dans la végétation et si, dans chaque série, on a créé les divisions de contenances à peu près egales entre elles, si, enfin, on respecte scrupuleusement les arbres vifs dont la dimension est inférieure à celle qui a été fixée, les conditions de la production tendront a s'égaliser dans chaque division, et le rapport de chaque période finira par s'équilibrer dans l'avenir, autant que le comporte la nature des choses.

De la Réserve. - Il n'est pas inutile d'indiquer par quels moyens on peut constituer la réserve dans les forèts jardinées, car pour les communes cette mesure est de la plus haute importance.

Si la forèt est trop petite pour pouvoir comporter des divisions, le plus simple sera de laisser un certain nombre d'arbres dépasser la dimension fixée pour leur abatage. Quand les besoins naitront, il y aura une coupe de réserve que l'on exploitera en jardinant dans toute la forèt et dont l'importance dépendra de l'économie ainsi faite.

$\mathrm{Si}$, au contraire, la forêt jardinée a une certaine itcndue, on peut affecter un certain nombre de divisions, un quart par exemple, à fournir les coupes de riscrve et les laisser intercalées parmi celles qui fournisent les coupes ordinaires. 
Comme, en définitive, la création des divisions dans une forêt jardinée a pour effet de former autant de petites séries d'exploitation séparées, inảépendantes l'une de l'autre, et ayant chacune une production spéciale, il est beaucoup plus rationnel de masser ensemble les quelques divisions qui sont affectées à la réserve et de les considérer comme une série d'exploitation spéciale. Il y aura ainsi dans les forêts jardinées par divisions, comme dans les taillis, deux cantons, deux séries d'exploitation: l'une pour les coupes ordinaires, qui sera des trois quarts de la contenance; l'autre pour la réserve, qui sera du quart séparé sur le terrain. Rien n'empêche de créer dans celle-ci, si elle est d'une contenance suffisante, des divisions destinées à régulariser le jardinage, et de lui donner un plan d'exploitation spécial en comptant le volume disponible pour une période donnée.

Exemple. - Le caractère des aménagements de futaies jardinées est de présenter une grande simplicité à laquelle s'adaptent, avec fort peu de modifications, les cadres que nous avons donnés pour les taillis et les futaies pleines. Voici une sapinière de 120 hect. que l'on veut exploiter en jardinant de manière à produire des arbres de $1 \mathrm{~m}, 60$ de circonférence à 1 mètre du sol, et de telle sorte que le propriétaire ait une reserve du quart de la production. On a crié 
6 divisions d'environ 15 hectares chacune, et une division de 30 hectares que l'on destine spécialement à fournir les coupes de réserve, et on a fixé à 6 ans la périoule ou rotation du jardinage. Le comptage des arbres qui ont atteint ou dépassé $1^{\mathrm{m}}, 60$ de tour et des tiges dépérissantes ou hors d'état de prospérer jusqu’à la période suivante a donné:

Puur les 6 divisions de l'ordinaire . . . 2766 mce

Dont le $1 / 6^{e}$ est de . . . . . . 461

Pour la division de la réserve . . . . 730

A exploiter au fur et à mesure des besoins, suit par année moyenne. . . . . . 122

Le plan général dexploitation ne sera pas autre chose que celui-ci :

Plan général d'exploitation.

\begin{tabular}{|c|c|c|c|c|c|}
\hline ఏ & 客 & 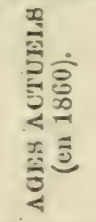 & $\begin{array}{c}\text { PERIODE } \\
\text { de } \\
\text { jardinage. }\end{array}$ & $\mid \begin{array}{c}\text { AGES } \\
\text { après } \\
\text { l'exploitation }\end{array}$ & 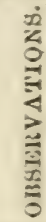 \\
\hline $\begin{array}{l}1 \\
2 \\
3 \\
4 \\
5 \\
6\end{array}$ & $\begin{array}{l}\text { A. - } \\
13 \text { - } \\
17- \\
1510 \\
1790 \\
14- \\
16-\end{array}$ & 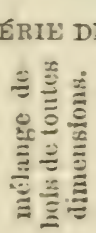 & $\begin{array}{l}\text { S COUPES ORDIAAT } \\
6 \text { ans : en ne cou- } \\
\text { pant que les bois de } \\
1 \mathrm{~m}, 60 \text { et au-dessus et } \\
\text { les brins morts ou } \\
\text { dépérissants. }\end{array}$ & $\begin{array}{l}\text { mélange de } \\
\text { bois de toutes } \\
\text { dimensions } \\
\text { au=dessous de } \\
1 \text { m,60 de tour. }\end{array}$ & \\
\hline $\mathrm{R}$ & $30-$ & $\begin{array}{l}\text { B. }-I \\
\text { id. }\end{array}$ & LA RESERVE. & id & \\
\hline
\end{tabular}


Dans de tels plans d'exploitation, il ne peut être question de révolution dans le sens habituel de ce mot (p.31). On peut constater que, dans cette forèt, les arbres de $1 \mathrm{~m}, 60$ de diamètre ont, en moyerne, 145 couches concentriques, et qu'elle sera ainsi exploitée à 145 ans; c'est tout ce qu'il est permis de déterminer.

Le plan spécial d'exploitation pour la rotation en cours sera le suivant:

Plan spécial d'exploitation

POUR LA PREMIÈrE PÉRIODE DE 1860-18ủ.

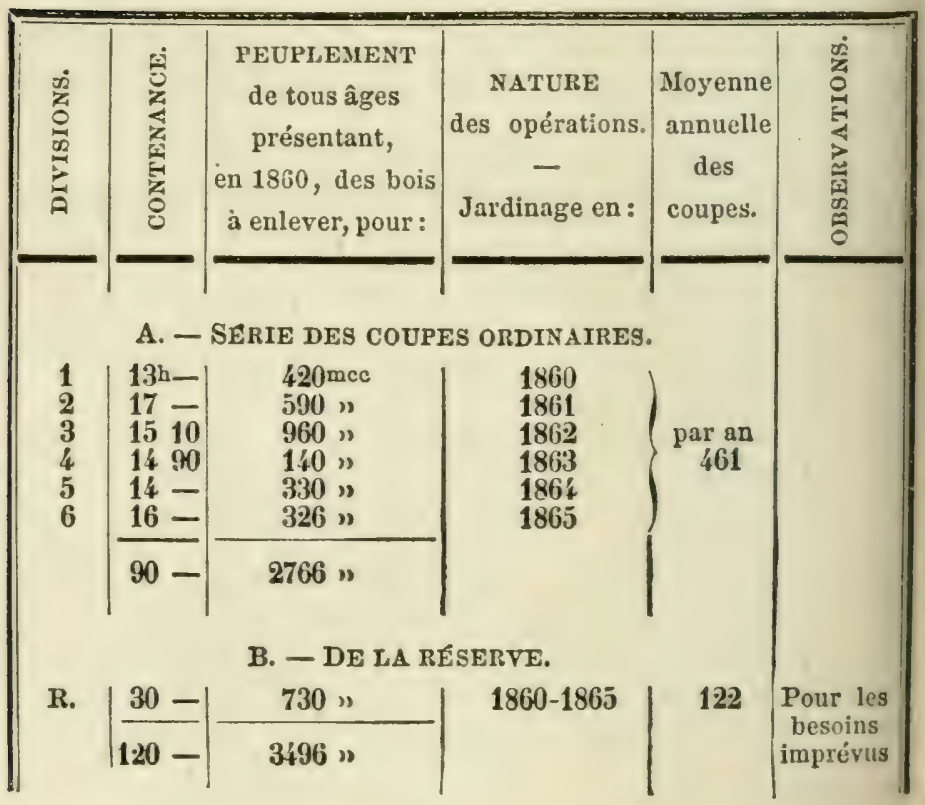


Compte d'Aménagement. - On tiendra pour la période du jardinage et pour chacune des séries créées dans la forèt le compte d'aménagement suivant le modèle que nous avons donné et qui est commun à tous les genres d'exploitation.

\section{Compte d'aménagenient.}

PERIODE SIX ANS, A PARTIR DE 1860.

Coupes de jardinage.

\begin{tabular}{|c|c|c|c|c|c|c|c|c|c|c|}
\hline$\ddot{2}$ & & & & & & & 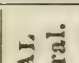 & $\begin{array}{r}\mathrm{B}_{1} \mid 100 \mathrm{I} \\
\mathrm{s}\end{array}$ & $\begin{array}{l}\text { lations } \\
\text { up }\end{array}$ & \\
\hline 产 & 1 & 2 & 3 & 4 & 5 & 6 & Eิ & 造 & 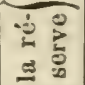 & $\begin{array}{c}\pi \\
z \\
z \\
0 \\
-1\end{array}$ \\
\hline 产) & $\begin{array}{l}\text { h. a. } \\
13 \text { - }\end{array}$ & $\begin{array}{l}\text { h. a. } \\
17-\end{array}$ & $\begin{array}{ll}\text { h. } & a . \\
15 & 10\end{array}$ & $\begin{array}{l}\text { b. a. } \\
1+90\end{array}$ & h. a & $\begin{array}{cc}\mathrm{b} & \mathrm{a} . \\
\end{array}$ & $\begin{array}{l}\text { h. a. } \\
120-\end{array}$ & h. a. & $\begin{array}{c}\text { h. a. } \\
30-\end{array}$ & 急 \\
\hline 总) & $\begin{array}{l}\text { m.c. } \\
420\end{array}$ & $\begin{array}{l}\text { m.c. } \\
590\end{array}$ & $\begin{array}{l}\text { m.c. } \\
960\end{array}$ & $\begin{array}{l}\text { m.c. } \\
140\end{array}$ & $\begin{array}{l}\text { m.c. } \\
330\end{array}$ & $\begin{array}{l}\text { m.c. } \\
326\end{array}$ & $\begin{array}{l}\text { m.c } \\
3 \text { i } 96\end{array}$ & $\begin{array}{l}\text { m.c. } \\
2766 \\
(451)\end{array}$ & $\begin{array}{c}\text { m.c. } \\
730 \\
(122)\end{array}$ & 0 \\
\hline 1860 & $40 b$ & & & & & & 405 & 405 & & \\
\hline 1861 & & $5 \% 0$ & & & & & 570 & 570 & & \\
\hline 1862 & & & 460 & & & & 460 & 469 & & \\
\hline 1863 & & & 500 & & & & 801 & 500 & 301 & \\
\hline 1864 & & & & 149 & 319 & & 468 & 468 & & \\
\hline
\end{tabular}

A l'expiration de chaque période, on établira le :ompte à nouveau en tirant un trait sous les chiffres Iẹ la période écoulée, et en inscrivant en tête les ré- 
sullats du momvan complige. Ide comple no contiendra pas, en géncial, de colomme pour les compes exIrandimaires, car il ne faut pas oublier que la rescrve n'est qu'une partie du revenu destiné a des besoins improvus, landis que les compes exlraordinaires sont des poulions de capilal qu’il est nécessaire d'exploifrer, mais dont il fant garder la note, parce que certaines personnes penvent on che comptables vis-avis dautres. Il est trop difficile dans les forèts jardimies de debrminer la possibilite pour songrer à demcler ce qui est revenu de ce qui est capilal surabondant. Ceprondant il peut se rencontrer des fo-

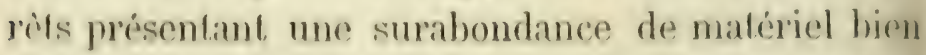
Cividenle. Dans ce cas, ce ne serait que par un calcul forl approximatif of analogue a colui qui a éle fart pour les fulates pleines it matériel surabondant qu'on pourail avoir une mesme des coupes extraordinares; celles-ci seraient inscrites en chillres hleus dans une colomme speciale du compte d'annénagement.

Comparaison avec la futaie pleine. - Ce mode de jardinage a, dians l'exploilation des forèts on futaies, des inconvenients si nombremx qu'on l'a géniralemont abandonme; mais il a certains avantages qui ne so renconfrent pas dims la méthode de la fulaic ploino et qui le fomt encore emploger, fimte do micux, dims cemtrimes cireonstances. 


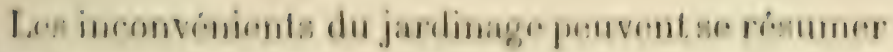
aiหsi :

1. Il produit moine en volume que la futair pleine,

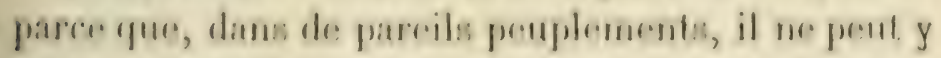
avoir uf celarecies ni produits intromediaires, dont l'imporlance dans les fublates pleines rest de 15 a 25

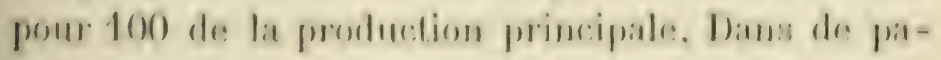
reile preuplements, on ne aat jamais si un hrin ac-

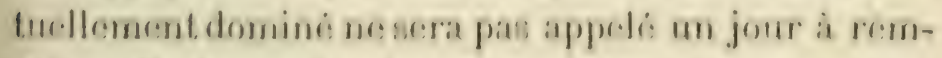

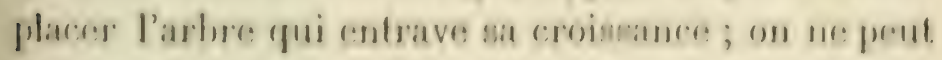

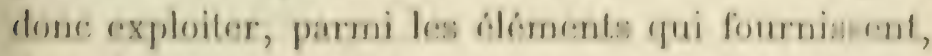

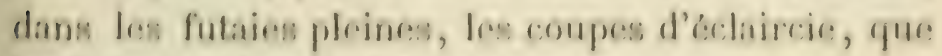
lese frims rentierement deprerienants.

D'un autre cote, les arbreu d'une forit jardinio sont assez souvent vicies ot mal venants; ils s'om-

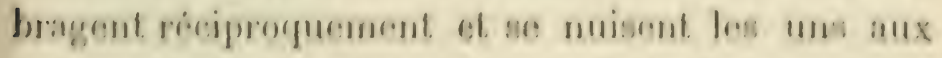
autres ; toutes ces circonstances font abainser notabement la production en volume, au point de ne la rendre mesurablo ro'i litre de rensergenement.

2" II rapporte moins en argent, cent-a-dire que lit ronte rest encores moins blevere ques aelle dese futaice pleines deja si banes; car, produisant moins en volume, et ayant un capital d'exploitation tout auns com iderable, le laux de placement s'abaieno naturellement dans une aseez forte proportion.

3" Linfin, l'exploitant n'ayant a son service aucune regle pour fixer la possibiliti merme d'une maniose 
approximative, ne pouvant la vérifier par des révisions décennales, est obligé à une grande modération dans ses coupes. Il est tenu à une circonspection dorit l'effet est de l'empêcher de recueillir tout l'accroissement de sa forèt sous peine de la détériorer.

Les avantages de ce mode ancien de culture forestière sont:

$1^{\circ}$ De présenter un sol continuellement couvert et régénéré, et de demander très-peu d'entretien, car on n'exploite jamais un arbre que quand il est déjà remplacé par un autre.

$2^{\circ}$ De se prêter facilement à l'exploitation de forèts de petite étendue, tandis que la futaie pleine exige des forêts ayant assez de contenance pour que chaque peuplement et chaque catégorie d'âges soient groupés sur une espace suffisant pour y receroir un traitement spécial. Dans le jardinage, au contraire, chaque hectare présentant une série complète, la contenance de la forèt peut descendre aux plus faibles limites.

Il en résulte que ce mode de culture est encore conservé :

10 Dans les pays de montagnes, dans les endroits exposés vents et aux frimas, où le forestier a des craintes sérieuses pour le résultat des coupes de régénẻration, dans des zones de défense, où l'on 
cherche moins à produire du bois que des abris pour d'autres forêts et d'autres cultures.

C'est ainsi que certaines forêts de hêtre et de sapin sont encore exploitées en jardinage même dans des montagnes d'assez faible élévation, ces essences supportant facilement l'ombre et le couvert. A de rrandes altitudes, les forêts de mélèzes et de pins ne peuvent être traitées que suivant ce mode. Dans es dernières conditions, où les semis naturels sont rès-difficiles et très-lents à se produire, on conserve oujours le massif boisé et on n'y exploite que quand a nature a produit des remplaçants en état de satisaire au but proposé.

2 o On jardine encore les bois de petite étendue ppartenant aux communes et aux particuliers et euplés d'essences qui ne repoussent pas de souche, omme le hêtre et le sapin, et dans lesquels les proriétaires trouvent ainsi, tous les ans, les bois de liverses dimensions dont ils ont besoin.

$3^{\circ}$ On emploie enfin le jardinage comme mode uxiliaire dans les combinaisons d'aménagement, omme moyen transitoire pour faire durer certains euplements déjà exploitables, mais que l'on a été bligé de classer dans des affectations intermédiai'es. Il permet, en effet, d'enlever les bois dépérisants au moment où ils perdraient de leur valeur, et le les remplacer par des jeunes bois qui entreticn- 
nent dans les massifs la vie et la durée. Nous en arons cité un exemple, page 115, pour la forèt de $H$.

Réglé et aménagé, le jardinage rend certains services qui ne sont pas à dédaigner; appliqué au hasard et sans mesure, il produit des forèts affreuses: n'y a plus d'exploitation raisonnée! 


\section{QUATRIĖYE PARTIE.}

\section{Détermination de la Possibllité d'une forêt.}

Définitions. - Nous avons appelé possibilité la quotité des produits que l'on peut retirer annuellement et d'une manière constante d'une terre exploitée dans des conditions données, sans la détériorer ou, en d'autres termes, sans en altérer la zubstance.

La terre n'a pas une possibilité abstraite et $a b$ solue : celle-ci dépend toujours du mode d'exploitaion auquel la terre est soumise. La culture, selon [u'elle est plus ou moins intensive, produit des réultats très-différents. Dans telle ferme, qui rend nnuellement tant de kilogrammes de blé et de: béail gras, on verra diminuer sensiblement les prouits, si le cultivateur vend les pailles et les engrais, 'il modifie le mode d'exploitation, l'aménagement 
de la terre. Telle futaie, bien entretenue, rapporte annuellement 5 à 6 mètres cubes de bois par hectare; si on abaisse successivement la rérolution, on voit la production décroitre jusqu'aux limites infimes des broussailles et des landes.

En sylviculture comme en agriculture, la possibilité dépend donc du mode d'exploitation dans le sens économique que nous arons donné à ce mot (p. 3), et le seul problème que l'on puisse se poser, est de chercher à déterminer la possibilitè relative à un mode d'exploitation donné.

Nous demandons pardon au lecteur de revenir sur la définition, et de préciser certains termes qui sont souvent employés l'un pour l'autre dans le langage usuel.

10 L'expression de la possibilité doit être annuelle, parce que les fruits de la terre se reproduisent tous les ans et que les besoins de l'homme sont soumis à la même loi. Cela ne veut pas dire que cette possibilité sera recueillie tous les ans; sa perception peut ètre annuelle, biennale, triennale, etc.

2 . La quotité des produits doit être à jamais constante, indéfiniment soutenue. Cette condition, inséparable de l'idée de possibilité, s'exprime par les mots : rapport soutenu, rendement constant.

Le rapport soutenu est donc la condition pour qu'un chiffre assigné à la production annuelle d'ın 
immeuble, exploitẻ dans des conditions déterninées, représente sa possibilité.

Dans le langage habituel, on confond sourent les mots: « revenus, fruits, produits, rente, rapport, production, rendement, ») et on leur donne, suivant les circonstances, des acceptions diverses. Dans le langage de la loi, comme dans celui de l'économie forestière:

Rendement, produits s'appliquent aux biens qu'on retire d'un immeuble d'une manière générale ;

Revenu, fruits, production correspondent à la possibilité telle que nous l'avons définie avec sa condition d'un rapport annuel et soutenu.

Ce n'est que lorsqu'on compare les produits au capital engagé qu'on emploie le mot rente. Celle-ci est la relation, le rapport qui existe entre le revenu t le capital engagé. La rente se mesure par un raport simple, le taux, qui est le revenu de cent uniés de capital.

Énoncé du Probseme. - Quand un propriétaire reut aménager une forèt, la première chose qu il loit faire, est de déterminer le but qu'il vent imorimer à son exploitation. 'Tel propriétaire veut-il rroduire des traverses de chemin de fer, qui exient des bois de 0m, 40 de diamètre, par exemple? tel utre veut-il limiter la production de sa forèt à des 
étais de mine et à des poteaux de télégraphe, qui comportent des arbres de $0 \mathrm{~m}, 20$ de diamètre? tel autre enfin, comme l'État, désire-t-il produire des arbres des plus fortes dimensions que comporte la végéta.tion de l'essence dont est peuplée la forêt? On constate queles dimensions de $0 \mathrm{~m}, 40$, de $0 \mathrm{~m}, 20$ correspondent, par exemple, à 130 ans, à 50 ans ; que les proruits des plus fortes dimensions sont obtenus dans la localité à 180 ans : 130, 50, 180 années seront. les révolutions choisies, les termes d'exploitabilité qui imprimeront à l'exploitation de la forèt son caractère, son but déterminé.

Si la forêt possède un matériel d'exploitation conslitué pour le but voulu, c'est-à-dire si tous les peuplements se suivent gradués en volume, en consistance et en âge, sans lacune et sans accident, la possibilité sera bien exprimée et fournie, autant que la nature des choses le comporte, par les procédés d'aménagement que nous arons indiqués. Mais le cas sera rare: le plus souvent, le propriètaire élère ou abaissc la révolution, en modifiant le but de l'exploitation. Le revenu, tel que les méthodes d'aménagement le déterminent, ira en s'améliorant prộressivement, si le capital est insuffisant; en diminuant, s'il est surabondant.

Fixer un âge pour l'exploitation d'une forèt donnée et chercher en mème temps à déterminer'sa possibilité 
dans le sens d'un rendement constant, indéfiniment soutenu, est donc un problème insoluble. Cie problème n'a de solution que dans le cas unique, exceptionnel, où le matériel d'exploitation coïncide exactement arec l'àge fixé pour l'exploitation.

Aussi les aménagistes ne s'y sont pas mépris, et les méthodes d'aménagement que nous arons exposées ne tendent à assurer le rapport soutenu que dans les limites d'un certain laps de temps, d'une période donnée. Quand on convertit un taillis en futaie, on sait bien qu'après la transformation la possibilité sera plus du triple de celle de la forêt en conversion. On se borne à déterminer la possibilité pour une période d'années, en combinant les divisions de la forêt en affectations, pour que, dans l'arenir, e revenu satisfasse le plus possible à la condition du rapport soutenu.

Tel est l'objet limité des méthodes qui consistert déterminer la possibilité dans la confection d'un ménagement ajant un but déterminé, tracé à l'afance. Tel est le sens de la condition du rapport souenu, à laquelle on s'efforce de satisfaire: on cherclie la ossibilité d'une période arec toutes les combinaisons maginables pour rendre, dans l'arenir, cette possijilité exacte tant que subsistera le but que l'aménarement avait en vue.

Mais très-souvent et tant que le but de cet ami- 
nagement n'est pas atteint, cette condition du rapport soutenu fait défaut d'une période à l'autre (v. p. 110). Parfois il dépend des convenances du propriétaire d'échelonner sur l'avenir le volume extraordinaire qu'il pourrait recueillir (l'État ou les communes, par exemple), ou de le réaliser dans un intervalle de temps plus ou moins court (la majorité des particuliers). Il ne dépend jamais du propriétaire d'augmenter immédiatement le produit d'une forêt pauvre en matériel, comme peut le faire un agriculteur pour une ferme délabrée en achetant des engrais et en augmentant le bétail du capital d'exploitation de sa culture. A l'agriculteur, il suffit d'avoir de l'argent; au sylviculteur, il faut de l'épargne et du temps.

Le problème qui consiste à déterminer la possibilité pour un temps donné dans une forêt aménagée suivant un but donné a donc fait l'objet des chapitres précédents; c'est une des questions, et mềme une question assez accessoire, de l'aménagement luimême: nous n'arons pas à y revenir.

Le problème qui nous occupe est tout autre:

Une forêt quelconque étant donnée, il s'agit de déterminer sa possibilité, c'est-à-dire la quotité des produits que l'on peut en retirer annuellement, d'une manière soutenue, sans la détériorer.

Utilitè. - Cette recherche a-t-elle une certaine 
utilité pratique, ou n'est-elle qu'une pure conception théorique et spéculative?

Il suffit, pour y répondre, d'indiquer les cas où la loi elle-mème en fait une règle de jouissance.

L'usager d'une forêt ne peut exiger que la possibilité de l'immeuble soumis à son droit, et le propriétaire peut toujours réduire sa jouissance suivant l'état et la possibilité de la forèt (G. for., art. 65, 112 et 121).

Les communes peuvent exiger de l'administration forestière la possibilité complète de leurs bois, mais ne peurent prétendre à rien au delà (C. for., art.112).

En cas de contestation, l'état et la possibilité des forèts sont réglés, suivant les cas, par les tribunaux civils ou les tribunaux administratifs (C. for., art. 65 et 120$)$.

L'usufruitier obligé par le Code civil (art. 578) à respecter la substance de l'immeuble grevé doit limiter sa jouissance aux fruits, c'est-à-dire à la possibilité de la forêt, telle que nous l'arons définie. Or combien sont nombreuses les situations ascimilées par la loi à celle de l'usufruitier? jouissance par suite de conventions matrimoniales, jouissance légale du père ou de la mère sur les biens des enfants, envoi en possession par suite de succession, d'aljsence, etc., tels sont les cas principaux.

Il est donc fort utile de rechercher comment il 
sera procédé quand, les intérèts adverses étant en présence, on voudra régler la jouissance à l'amiable ou judiciairement.

Solution. - Les méthodes d'aménagement que nous arons expliquées renferment implicitement la solution du problème.

Écartons d'abord, cependant, les forêts jardinées. Ce que nous arons dit, p. 161, sur la possibilité des forèts jardinẻes montre suffisamment qu'il n'y a nul mojen pratique d'arriver à une détermination raisonnẻe de cette possibilité. Il pourra y aroir des évaluations dues à l'expérience, à des comparaisons avec des forêts voisines; mais il n'y aura que cela. La solution sera tout entière dans l'expérience et dans le choix du forestier chargé de l'expertise.

Ce n'est que dans les forèts traitées en taillis et en futaie pleine que la solution est possible, et encore faut-il remarquer que la solution comporte une certaine latitude; tout ce que l'on peut dire, c'est que la solution repose sur une pratique raisonnée.

Voici comment il peut être procédé: Supposons une forèt de 120 hectares qui forme la dọt d'une femme mariée, et dont le mari veut faire déterminer la possibilité pour faciliter dans l'avenir la liquidation de sa communauté.

On fera l'analyse et le parcellaire des peuplements, 
pour la révolution de 110 ans, terme d'exploitabilité qu'il fallait avant tout déterminer.

Cette détermination faite, on fixe la durée des périodes d'après les données culturales de la localité, par exemple 5 périodes de 22 ans; on combine les

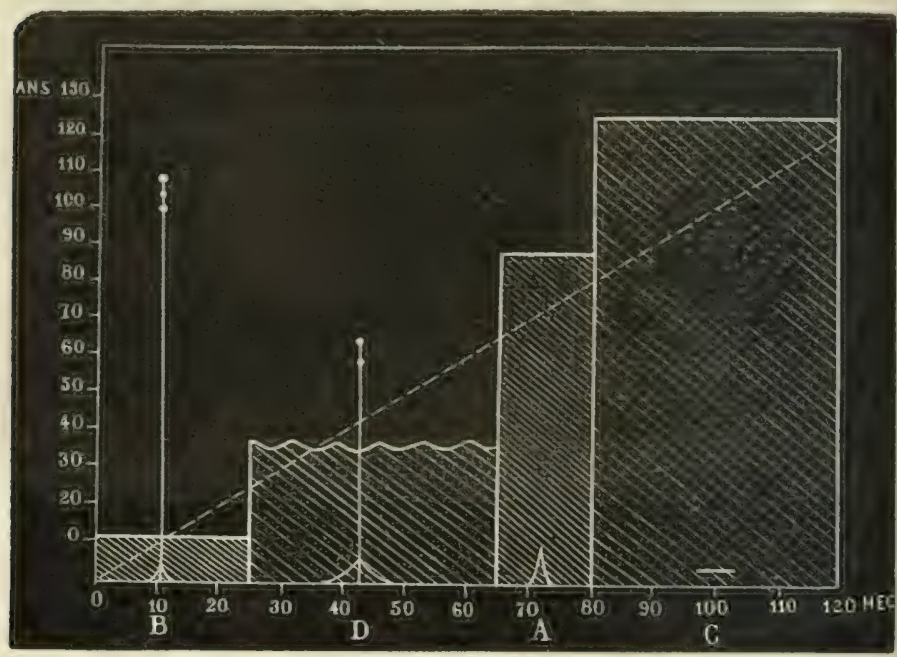

Fig. 5. - Combinaison d'aménagement.

parcelles dans les affectations de telle sorte que celles-ci soient aussi équiproductives que possible; on compte et on cube le volume de la première affectation; on applique, en un mot, la méthode d'aménagement dont nous avons fait l'exposé; et la possibilité ainsi déterminée par cet aménagement sera la solution du problème. L'exactitude de cette solution dépendra du suin avec lequel on aura procédé à tous 
les détails de l'opération et surtout à la confection des affectations.

La difficulté est de tracer la ligne moyenne compensatrice qui délimite un matériel d'exploitation équivalent à celui que représente la forêt. Cette difficulté s'accroît quand il y a des inégalités ou des lacunes considérahles dans les âges du peuplement. C'est une affaire d'expérience et de tact forestier; on y arrivera par un tâtonnement inspiré de la connaissance des lois de la végétation et aidé, au besoin, d'une construction graphique ${ }^{1}$. Mais il ne faut pas s'y méprendre, cette construction graphique ne fournira pas, à elle seule, la solution du problème; car la figure ne présente, en dimensions exactes, que deux des éléments qui entrent dans la constitution d'un matériel d'exploitation : l'àge et la contenance. Le troisième élément, l'état de végétation, c'est-àdire la consistance et le rolume des peuplements, n'y est exprimé que par de simples indications. C'est au forestier à saroir tenir compte de cet état de régétation et des ressources de la forêt: il s'aidera de tables de production, d'exemples choisis dans la localité, pour fixer ainsi cette ligne de compensation et déterminer, par suite, la possibilité de la forèt.

Pour un taillis composé, on fera la mème opéra-

1 Tons les géomietres comaissent ce problime du 3e live de Legendre qui sert ì remplacer une ligne brisée par une ligne droite équiralente. 
tion en ce qui concerne le taillis. Quant à la futaie, on comptera les arbres par catégories sur chaque coupe, on comptera également les futaies qui ont été exploitées dans les coupes oủ les souches sont encore visibles, et on en déduira, autant que cela est possible, le plan de balivage.

Il est évident que nous ne faisons qu'indiquer ici des méthodes que l'expérience et la pratique d'un forestier peuvent seules savoir appliquer. Chaque fois qu'il s'agit de faire l'application d'un procédé, fût-il le mieux raisonné, il faut, avant tout, être homme du métier.

Autre problème. - Mais la question peut être plus compliquée: à la mort d'un chef de famille, on peut vouloir déterminer la possibilité qu'avait la forèt au dẻbut de la communauté, pour fixer la valeur des reprises matrimoniales. Pendant la gestion d'un usufruitier, le nu-propiétaire peut s'alarmer de certaines coupes opérées dans la forêt grevée et vouloir vérifier la possibilité dont la forêt était capable avant les exploitations de l'usufruitier. Il s'agit de déterminer la possibilité qu'arait une forêt quand de nombreuses coupes y ont été faites, quand plusieurs années se sont écoulées et quand aucun inventaire n'a été dressé, aucune comptab.lité n'a été tenue.

De pareilles questions se sont posées plus d'une 
fois devant les tribunaux; mais ce serait se montrer bien exigeant envers la science forestière que de lui demander un procédé exact pour la solution d'un tel problème. La donnée elle-mème n'est-elle pas une inconnue? Tout ce qu'il est possible de faire, c'est de reconstituer, tant bien que mal, l'état ancien de la forèt en visitant les parties exploitées, en les comparant avec les parties roisines, en s'aidant de tous les moỵens possibles. Si on y parvient, si les documents sont assez certains et s'il ne s'est pas écoulé un temps trop long, on rétablira un parcellaire fictif de l'état ancien de la forèt. On procèdera alors comme il vient dètre dit, et la comparaison du chiffre de possibilité ainsi trouré arec celui des exploitations faites fournira le montant des reprises ou des répétitions entre les parties.

Ciest le seul conseil pratique rui puisse ètre donné : le plus sourent, la question est insoluble. Aussi ces répétitions sont-elles la source la plus fréquente des désunions de famille, des procès les plus irritants. C'est pour les éviter qu'un propriétaire soncieux de l'asenir, désireux surtout de ne point laisser après lui une cause de discorde, fera bien daménager ses forèts et d'en tenir la comptabilité sur des bases analogues à celles qui ont été exposées dans ce petit traité. 



\section{APPENDIGE.}

\section{Gestion d'un ensemble de Forêts.}

On a souvent agité la question de savoir quels sont les procédés de comptabilité, les méthodes administratives qui doivent être employés dans une gestion importante de forèts. Nous n'avons point la prétention le traiter en quelques pages cette délicate question, laquelle tiennent des intérêts de premier ordre. Nous nous bornons à indiquer ici ce que nous avons iu, ce que nous avons expérimenté: c’est, à notre sens, un sujet digne d'études et de réflexions.

Nous arons visité, en 1872, l'importante gestion les forêts de la famille de N..., que nous avions léjà examinée en 1865̃. Le but de notre visite était, on d'étudier ces forèts au point de vue cultural, ni l'examiner leur mode de traitement, mais de nous clairer sur les méthodes administratives emplogées lans la gestion de ce vaste ensemble de propriétés at de forêts. Les domaines yue cette famille a eu le sage sprit de faire échapper au partage des générations 
successives, en les conservant dans l'indivision, sont gérées pour le compte des différentes branches par M. le duc de N...., chef actuel de la famille. Ces forêts sont situées en diverses localités deLuxembourg: de Suisse et de France. Elles ont été aménagées en 1860 par des agents forestiers, anciens élèves de l'Écoleforestière de Nancy; elles ont une contenance qui dépasse 30,000 hectares et qui atteint ainsi la moyenne des forêts domaniales d'une de nos conservations forestières.

M. le duc de N... se mit à notre disposition avec une complaisances extrême, et, nous conduisant dans son cabinet dont le mur était orné d'une grande carte de l'état-major piquée de quelques épingles à têtes rouges et noires : "C'est d'ici, nous dit-il, que je puis diriger la conduite de nos exploitations de forêts.

"Mon personnel administratif est réduit au strict nécessaire: un administrateur qui opère seul, sous sa responsabilité, dans l'étendue de sa circonscription - voilà l'élément local de la gestion; - quelques vérificateurs ambulants que j'envoie sur les différents points et dont je suis toujours la marche au moyen des épingles piquées sur la carte d'ensemble que vous voyez - voilà le système de contrôle.

“Jen'ai pour gardes que des ouvriers forestiers qui sont réunis sous la conduite d'un chef de chantier, 
ourrier comme eux, et qui sont engagés à l'année et payés à raison de 3 ou 4 fr. par jour. Ce còté de ma gestion peut paraitre coùteux; mais, à notre époque, les ourriers ] uicherons tendent à quitter les forêts pour le travail plus rémunérateur des vilips et des manıfactures. D'un autre còté, il est pénible de placer sans cesse entre le devoir et la tentation, des gardes dont le traitement est plus que modeste. Tout compte fait, j’ai un arantage pécuniaire à aroir toujours sous la main des ourriers expérimentés qui font mes travaux sans intermédiaire d'entrepreneur. Mes ouvriers exploitent mes coupes, sauf celles dont les bois de service assurent toujours la rente sur pied, font les travaux de terrassement, de plantation, d'entretien cie routes, etc. Sur chaque journée de travail, une ou deux heures sont consacrées à la surveillance, à divers moneits et selon les circonstances. Le trarail est soumis à un rèclement analogue à celui des établissements industriels; le: zèle des ourrier's est excité par des gratifications quand les traraux sont faits dans les délais fixés. Enfin, mes brigades ourrières sont, à certaines épountues, employées aux travanx agricoles de nos terres voisines des forèts. L'agriculture a des points de contact si fréquents arec la Hiculture gu'une gestion sagenent entendue troure tonjours a se compléter par l'une et par l'autre: tout \& tient dians les interrets de la terre. 
( L'aménagement des forèts forme la base de mon système d'administration. Sans aménagement, je considère qu'il n'y a pas de gestion raisonnée de forèt. C'est lui qui est le contrat de gestion entre le propriétaire et l'administrateur de chaque circonscription; c'est lui qui permet seul de donner à ce dernier cette liberté de conduite qui, fondée sur la responsabilité, élève l'homme au-dessus de la condition d'un simple instrument et l'intéresse à son œuvre. C'est l'arnénagement seul qui permet de contrôler sa gestion et de donner au vérificateur un point d'appui autre que des appréciations personnelles toujours contestables.

« La difficulté était de permettre la concentration et le contrôle; elle portait donc sur trois points: l'aménagement, sa comptabilité, et les comptes de gestion.

«L'aménagement devait avoir un système unique, uniforme, se pliant à la vérification et au contròle des résultats. La méthode simplifiée si heureusement introduite en France par MM. Lorentz et Parade et si bien enseignée à l'École de Nancy s'adapte fort bien à cette condition administrative. Quelles que soient les localités et les exigences culturales, le cadre d'aménagement peut ètre uniforme. En effet, dans les taillis, la coupe annuelle n'est-elle pas une affectation correspondant à une période d'un an? 
Dans les futaies, les parcelles ne correspondent-elles pas aux particularités culturales permanentes? Le groupement des parcelles en affectations, la fixation des périodes, plus ou moins long̣ues selon les difficultés du repeuplement, la détermination de la révolution selon le but de l'exploitation n'assurent-ils pas toutes les conditions économiques et culturales? On peut les fixer, les modifier selon les besoins, sans que le cadre mème de l'aménagement soit modifié. «La seconde difficulté était d'assurer le contròle matériel, effectif, des aménagements au moỵen d'une comptabilité simple et susceptible de centralisation. Elle a étè vite résolue en écartant des reg̨istres de contrôle tout ce qui concerne la comptabilité des ouvriers, des ventes, des travaux, tout ce qui est relatif à la vérification des procédés d'estimation, cout ce qui a trait, enfin, à l'administration mème. C'est l'oljet de livres auxiliaires et un sujet étranger la marche de l'aménagement. Ainsi réduits, les comptes d'aménagement permettent de voir, à tout noment et au premier coup d'œil, où l'on en est Aans la marche de l'aménagement et comment il est ippliqué.

«Le còté moral et administratif de la gestion de haque forèt a été assuré par une comptabilité spéiale ou plutòt par un état récapitulatif des recettes it des dépenses de lannée. Il fallait se défentre de 
ces comptes rendus généraux dans lesquels les régisseurs ne manquent jamais de se décerner des iloges plus ou moins déguisés. Je n'accepte que les comptes de chiffres et de résultats qui permettent seuls les comparaisons et les études.

“Voilà l'exposé de mon système administratif; en voici maintenant la pratique sous le rapport des trois chefs que je viens de rous indiquer:

« $1^{\circ}$ Les procès-verbaux d'aménagement de chaque forêt sont résumés en trois états ou tableaux, dont voici les formules imprimées.

«La première formule indique les renseignements généraux de l'aménagement et contient le tarif de cubage adopté pour la détermination de la possibilité, pour les estimations et les rérifications.

«La seconde est relative au plan général de l'exploilation. Avec de légères modifications elle convient tout aussi bien aux forèts de taillis qu'aux futaies pleines et aux futaies jardinées.

“ La troisième concerne le plan spécial d'exploitation pour les futaies. Naturellencnt cette formule est sans utilité pour les taillis, puisque la période n'y existe pas.

«Les résumés contenus dans ces tableaux n’ont pour lut que de présenter d'une manière saisissante les chiffres qu'il est toujours indispensable d'aroir sous la main. Ils ne dispensent nullement de l'étude 
du procès-verbal d'aménagement, dont l'expédition complète est remise au régisseur local.

\section{FORÊT de}

appartenant à

\section{Série No}

\section{dite de}

\section{CONTENANCE :}

\section{Mode de traitement 1 .}

Révolution :

ans.

Période :

ans.

Nombre d'affectations ${ }^{2}$ :

Matériel d'exploitation ${ }^{3}$ :

Année de l'application de l'aménagement.

Traits généraux de l'aménagement ${ }^{4}$ :

\section{Tarif de cubage.}

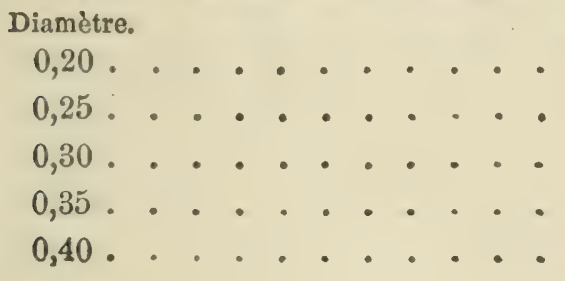

1 Taillis, ou taillis composé, futaic pleinc ou futaie jardinée.

2 Pour les taillis, le mot affectation est remplacé par coupes.

3 Indiquer si le matericl d'exploitation est constitué, surabondant on insuffisant, si la forêt est en conversion.

+ Indiquer sommairement le caractère géuéral de l'aménagement, le but principal ì atteindre. 
Plan général d'exploitation.

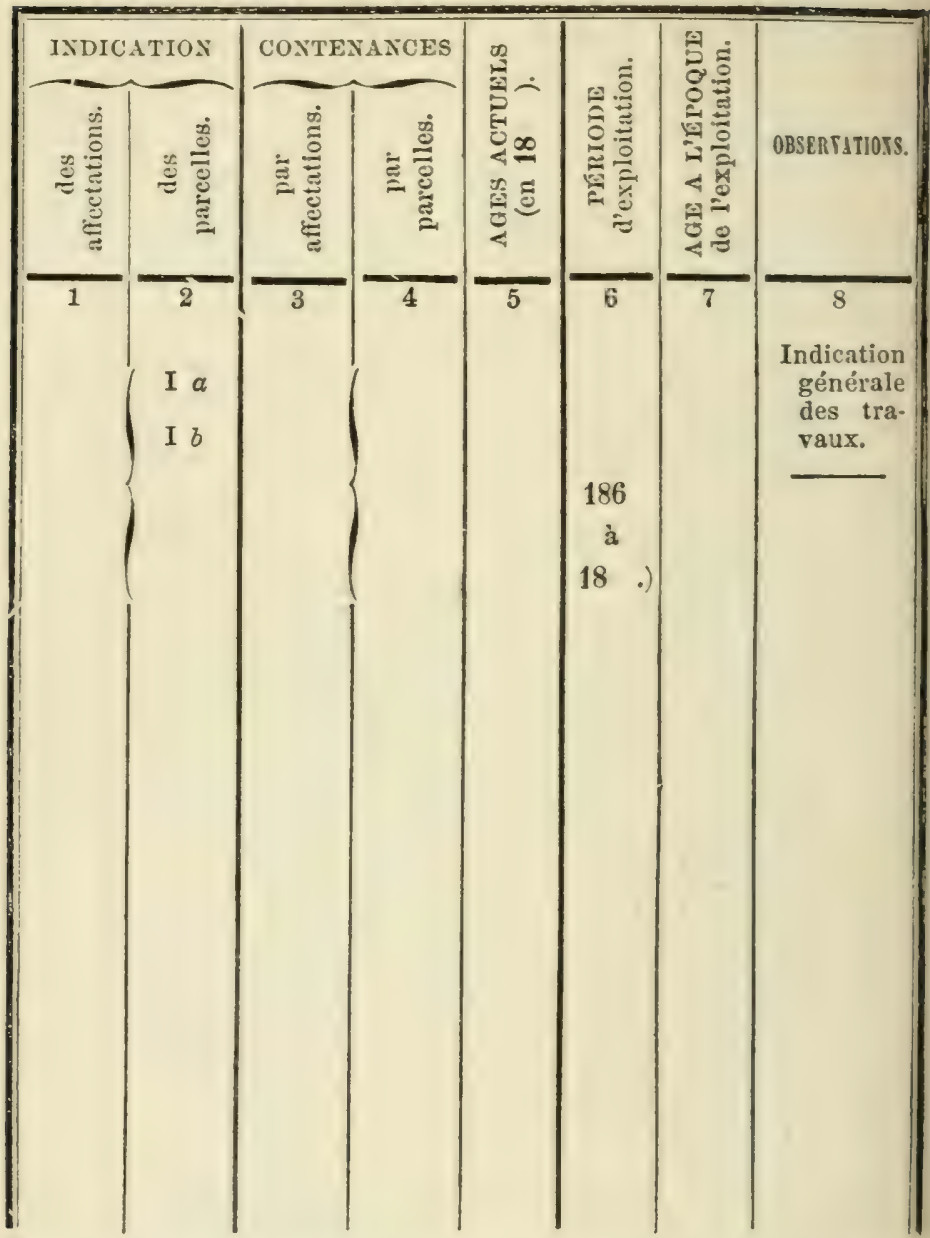

Pour les taillis, les colonnes 2 et 4 restent en blanc. Pour les taillis comporés, on met en tête de la colonne 8 le plan adopté pour le barsivage 
Plan special d'exploitations.

POUR IA PREMIÈRE PÉRIODE DE 186 à 18 .

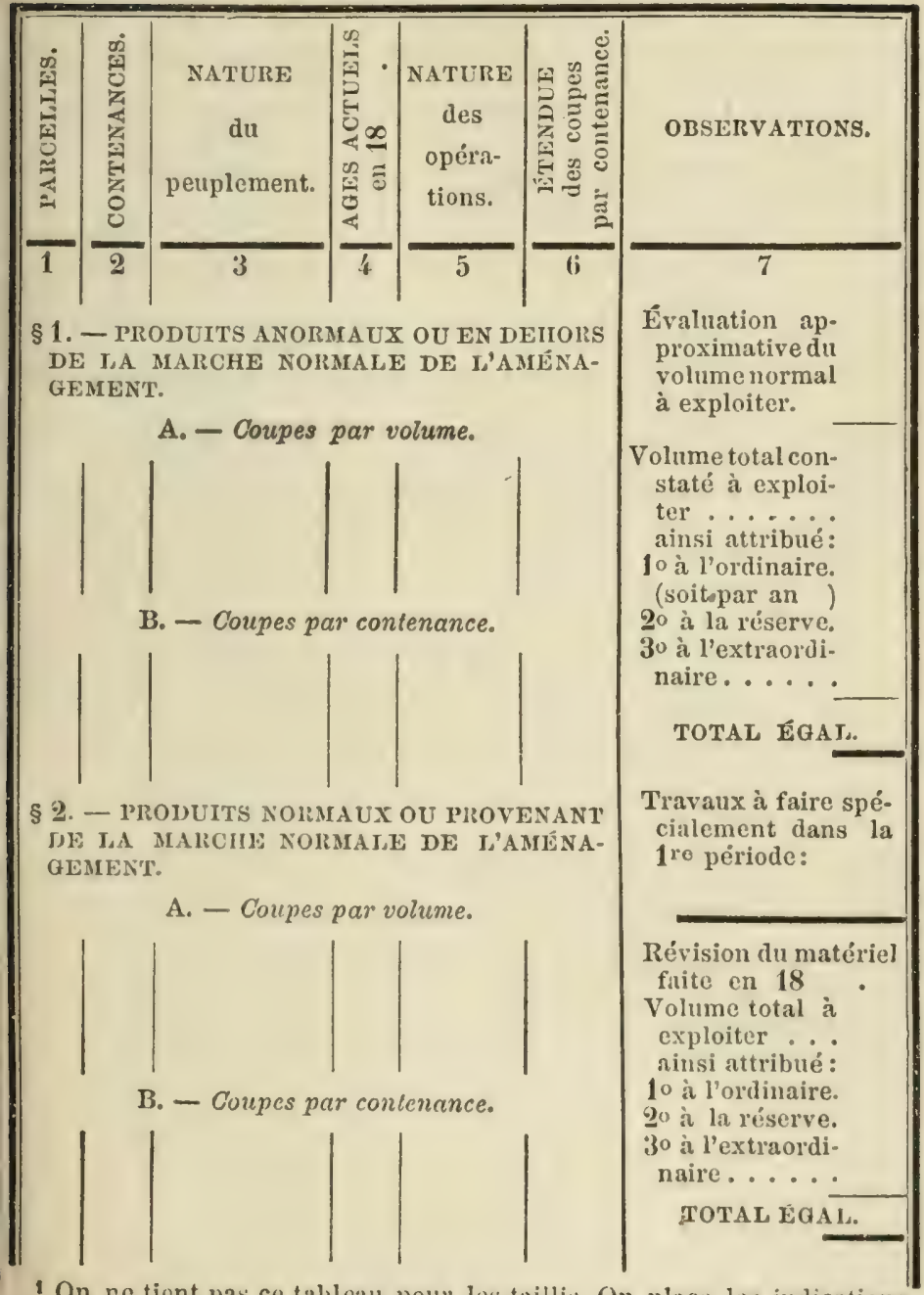

I On ne tient pas ce tableau pour les taillis. On place les indications de la colonne 7 dans le tableau du plan général d'exploitation, qui est alors le senl à tenir. 
« $2^{\circ}$ La comptabilité destinée à constater, chaque année, la marche de l'aménagement n'a qu'une formule unique, uniforme, s'adaptant à tous les modes de régime et de traitement : taillis, futaies, jardinages, à l'aide de modifications très-légères. En réalité, c'est un compte de doit et avoir ouvert à chaque période et à chaque parcelle; la forme seule diffère de celle admise généralement en comptabilité, dans le but de présenter aux regards les situations d'une manière plus frappante. Un compte est ouvert à chaque suite de coupes que comporte l'aménagement. Ainsi, dans les taillis composés, il y a ordinairement deux comptes, l'un pour le taillis, l'autre pour la futaie; dans les futaies pleines, il y en a deux ou trois; dans les conversions, leur nombre est plus considérable encore. On se sert d'encre noire pour les coupes ordinaires, rouge pour les coupes de réserve, bleue pour les coupes extraordinaires. Les chiffres des volumes ou des contenances sont ceux qui résultent des procès verbaux d'estimation ou d'arpentage, selon que les coupes ont été vendues sur pied ou après façon. 


\section{Compte d'aménagement.}

PÉrIODE DE 186 à 18 .

Coupes par (1)

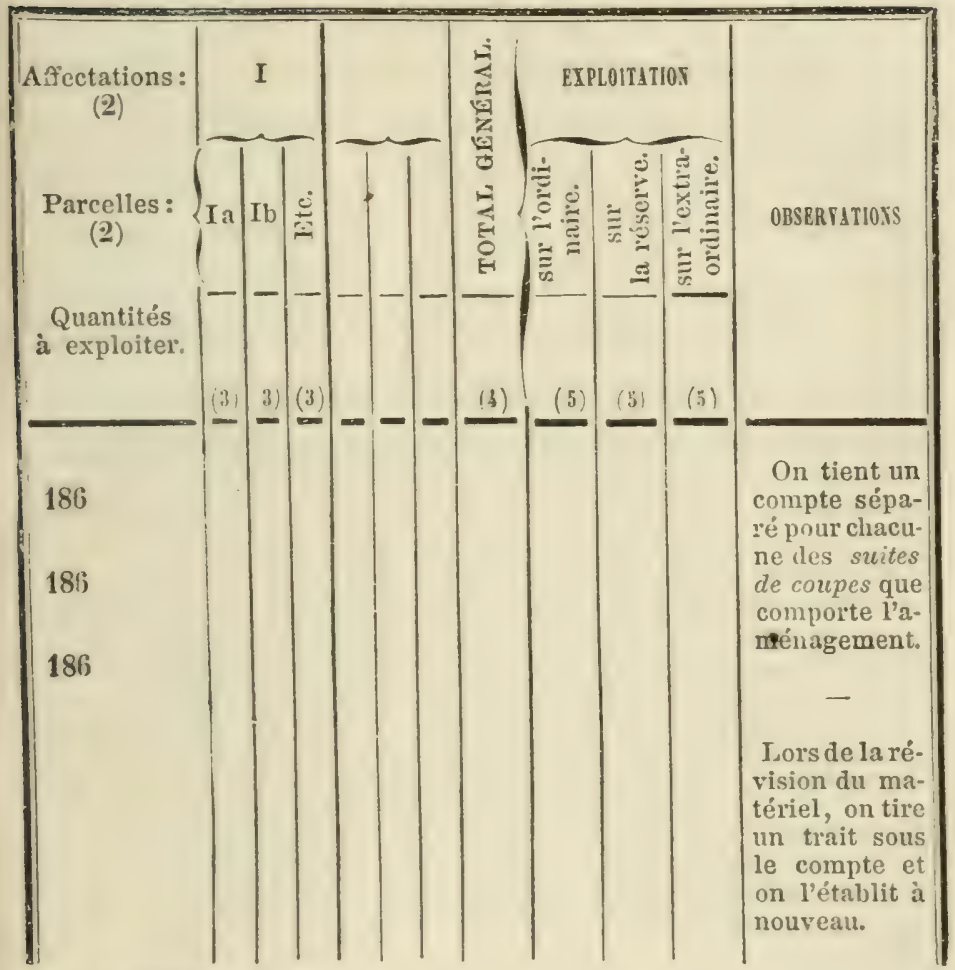

(1) Contenance ou volume.

(2) On ne doit porter que les affectations et les parcelles où des coupes doivent se faire d'après le plan spécial de la période. Pour les taillis, les affectations se remplacent par les coupes.

(3) Indiquer en volume ou en contenance le chiffre i exploiter selon qu'il est un volume ou une contenance.

(4) Cette colcune fournit par conséquent les renseignements suivants : en tête, le total des volumes ou de's contenances à expluiter; dans la co- lonne, le total par chaque année des quantités exploitées.

(5) On indique les chiffres attribués a chacun de ces comptes par le plan spécial (colonne d'observations) pour les aroir toujours sous les yeux. On a soin de rappeler si l'accroissement entre daus le chiffre de la réserve. 
( $3^{\circ}$ Enfin, le compte moral et administratif comporte deux états, l'un pour les recettes, en matière et en argent, l'autre pour les dépenses.

« Le compte de recettes contient les exploitations non prévues par l'aménagement : chablis, bois morts, coupes dans les parties de forêts en dehors des prescriptions ou des prévisions de l'aménagement. Il y a ainsi :

« Parmi les produits prévus : 10 les produits principaux, qui sont ceux qui entrent dans le calcul de la possibilité principale par contenance ou par volume; $2^{\circ}$ les produits intermédiaires, dus aux coupes de nettoiement ou d'éclaircies.

" Parmi les produits imprévus, il n'y a pas lieu de distinguer entre les produits intermédiaires et principaux; il n'y a plus que des produits accidentels.

( Enfin, sous le rapport de la recette en argent, les produits accessoires sont ceux qui proviennent de la chasse, de la pêche, du pàturage et des concessions autres que le bois.

« Les coupes par contenance sont indiquées à la fois par leur superficie et par leur volume, ce dernier résultant des procès-verbaux d'estimation ou de dénombrement, selon que les coupes ont été vendues sur pied ou après façon. 


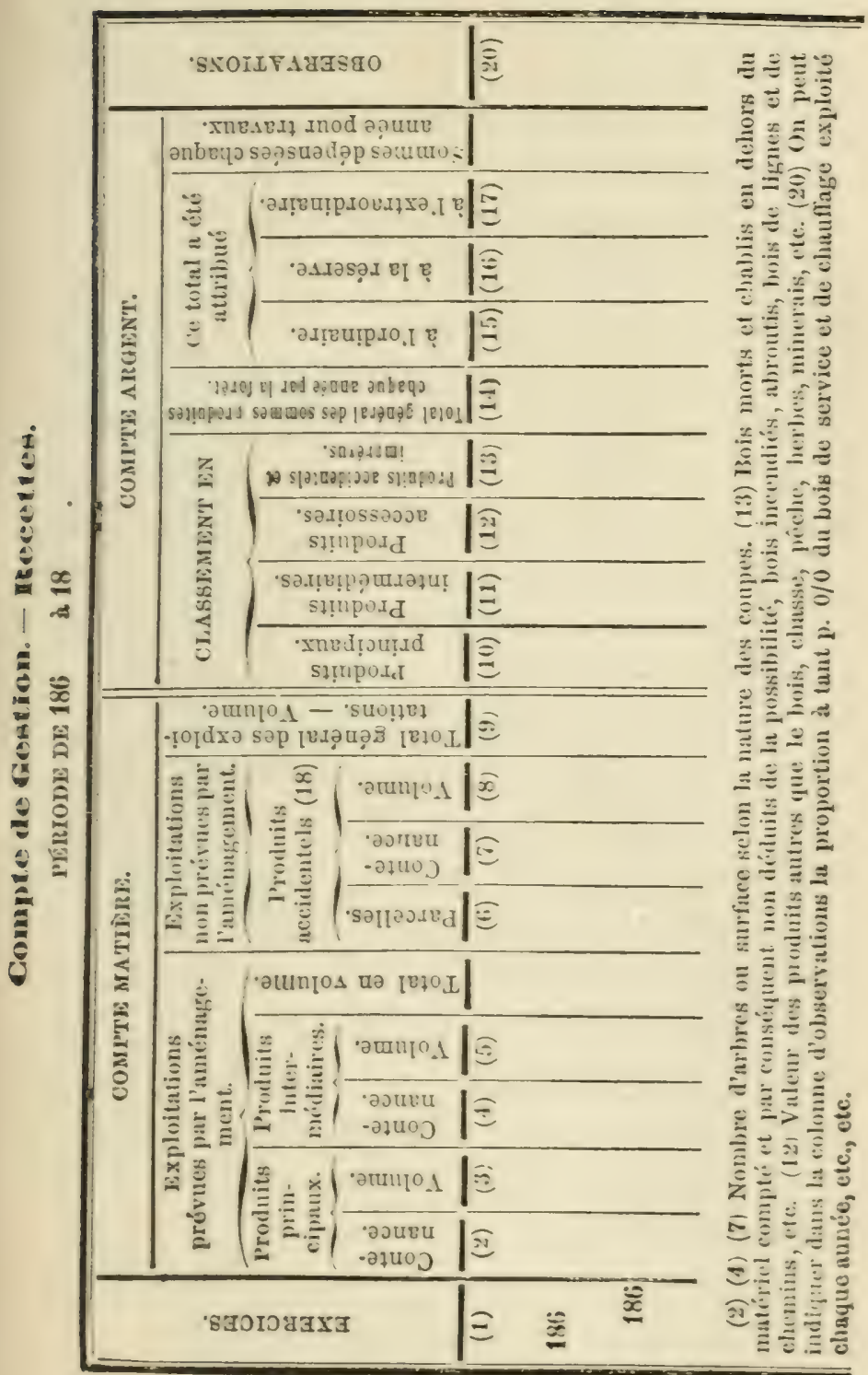


“ L'état des dépenses ne comprend que celles qui ont un caractère spécial à chaque forèt; ce sont celles qui dépendent, en quelque sorte, de la régie locale et qui permettent de la juger. Les dépenses judiciaires, celles du personnel-administrateur ont un caractère général et extrinsèque. Je puis les répartir proportionnellement à l'étendue et à l'importance de charque forêt; mais elles dépendent bien plus de moi et des circonstances extérieures que des administrateurs locaux, dont il faut apprécier la gestion. C'est pour ce motif que ces dépenses ne figurent pas dans le compte de gestion de chaque forêt.

« Le compte se termine par les résultats obtenus chaque année, c'est-à-dire le nombre de mètres cubes exploités par hectare, le produit en argent par hectare, et le prix moyen du mètre cube résultant de la division des produits totaux l'un par l'autre. A cet effet on retranche du produit brut en argent les sommes dépensées pour frais de garde, d'exploitation et d'entretien. Une colonne spéciale indique la dépense rapportée à l'hectare pour les traraux d'amélioration. Les frais de premier établissement se cumulent chaque année; ceux de l'entretien ne s'éraluent que par rapport à l'année qui leur a donné naissance. 


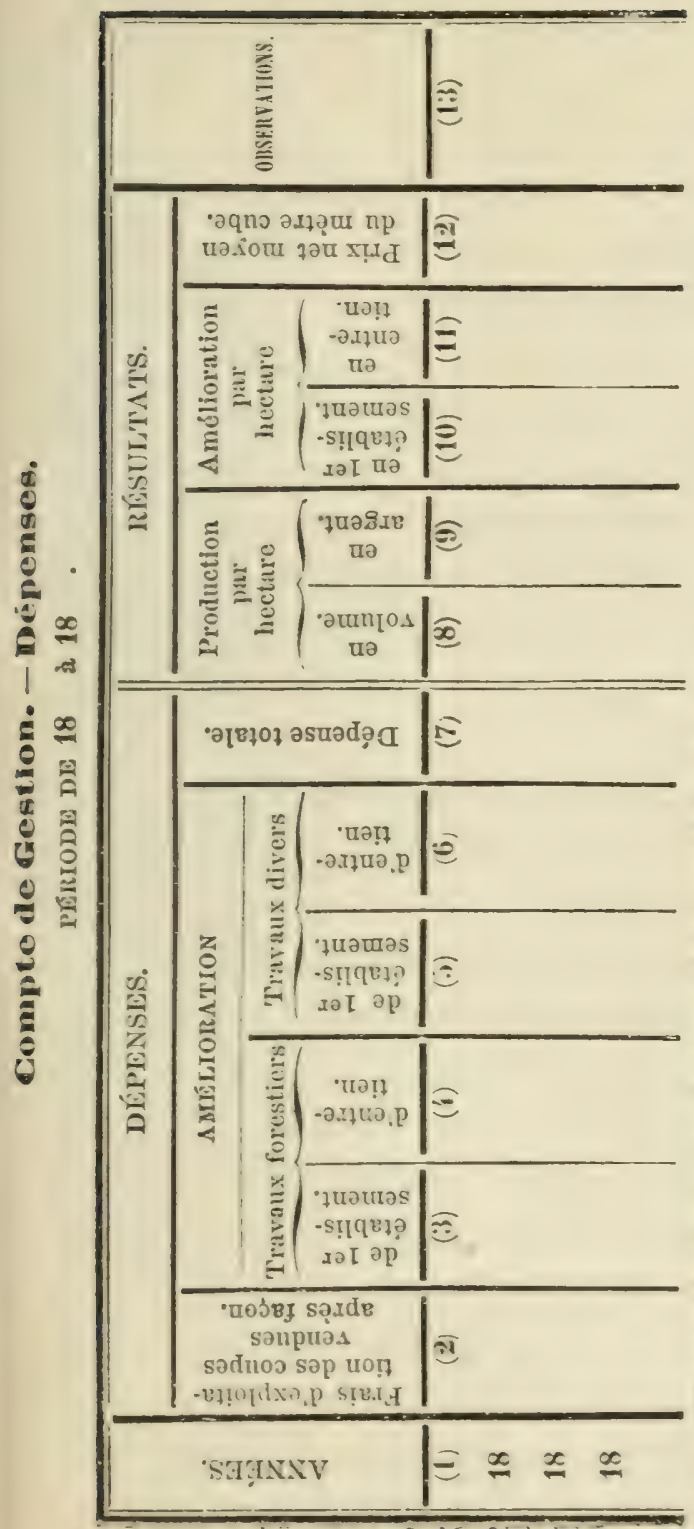

3
3
0

$\approx \frac{1}{3}$

غี

올

原 政

:

से

震

$\therefore$

อ

¿

政

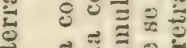

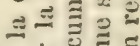

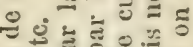

○㺃

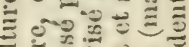

可

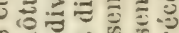

이을 - 90

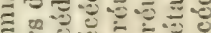

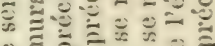
อ $\triangleq$ क ज्ञ

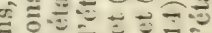
三

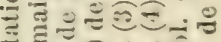

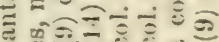
$\vec{a} \div-1$ 응 $\overline{3}$

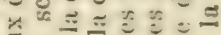

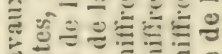

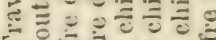
₹ -6อง

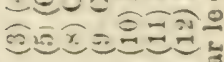


( Chacun de ces comptes ne demande que quelques lignes d'écriture par année, et je tiens d'un de mes administrateurs locaux, dont la gestion comporte plus de 6000 hectares, qu'il ne lui faut pas plus d'une journée à la fin de l'année pour relever les chiffres de ces divers états. Chacun fait, dans sa circonscription, ce relevé annuel, sur une feuille rolante qui est soumise au contrôle du vérificateur. Ce n'est qu'après le travail de celui-ci que les chiffres sont définitivement inscrits sur les registres tenus à la fois chez l'administrateur de la circonscription et chez le vérificateur. Réglés ainsi contradictoirement, ils forment la base indiscutable des vérifications ultérieures sur le terrain.

( 11 n'esi pas sans intérêt de vous faire remarquer, continua M. de N..., les résultats obtenus par ces procédés de gestion.

« Depuis que mes forêts sont aménagées, tout mon monde s'est mis immédiatement au courant des plans d'exploitation; les opérations ont acquis un degré de précision jusqu'alors inconnu; les travaux d'entretien et d'amélioration sont faits à leur lieu et place sans perte de temps ni d'intérêt; les coupes se font dans chaque affectation avec leur caractère propre, si bien qu'il suffit d'indiquer la parcelle par son numéro et son indice au plus humble de nos ouvrier's pour qu'il sache immédiatement la nature et le ca- 
chet particulier de la coupe qui doit s'y faire. Le personnel s'intéresse à son œuvre et s'attache à sa forêt.

( Voilà les résultats généraux.

«Quant au contròle des aménagements, rien n'est plus facile. Le vérificateur qui veut examiner l'état d'assiette annuel des coupes présenté par chaque administrateur local voit de suite, à l'aide des comptes d'aménagement, si l'on est dans la marche du plan d'exploitation, ce qui reste à exploiter dans chaque parcelle, à quelle époque la dernière coupe a été faite. Au moindre doute, il se rend sur les lieux. Les chemins de fer ne sont pas faits pour rien, et nos grandes sociétés de crédit ont fort bien compris les ressources des voies ferrées, en simplifiant leur organisation hiérarchique et en introduisant partout le contrôle ambulant.

«Tout, jusqu'à la conduite de l'administrateur local, peut être ainsi rérifié.J'ai toujours pensé que l'on devait pouvoir faire une forêt comme on fait la caisse d'un complable, avec les approximations que comporte la nalure des choses, bien ertendu. Ainsi, j'ai un vérificateur actuellement à X...; le compte de cette forèt porte que dans la parcelle Ic il y a $64-27$ mètres cubes; jusqu'à ce jour on y a exploité 1523 mètres cubes, c'est donc 4904 mètres cubes qui doivent rester sur pied. Une dépècle ì enroyer à 
M. Z..., et demain ou après-demain j'aurai le comptage de ce qui existe réellement dans la parcelle. On comprend combien ce système est de nature à tenir les hommes en éreil. Je n'admets pas qu'il y ait des régisseurs capables de faire des coupes, mème à mon profit, dans les parties de forèt non autorisées par moi en dehors de la marche de l'aménagement, et de manquer ainsi au contrat de gestion qu'ils ont accepté. Cela serait trop vite remarqué, et l'on est trop honnête, en France, pour manquer aux engagements pris. Je ne me mets en garde que contre les excès de zèle, qui ont trop souvent conduit à exagérer les coupes pour faire plaisir et argent au propriẻlaire.

«Le chiffre qui représente le prix moyen du mètre cube dans chaque forèt est toujours l'objectif de mes comparaisons et de mes améliorations. Rien n'est plus variable: de deux francs à peine que le mètre cube vaut sur place dans une forêt des montagnes du Jura, il varie partout et atteint 20 francs dans la forêt de X... dans le Luxembourg. Toute amélioration qui fera gagner au mètre cube quelques centimes dans une forèt deshéritée de chemins de vidange se traduira dans ma caisse par des chiffres considerables. Quand donc je fais mon budget et que je reux répartir la somme que je destine tous les ans aux améliorations, je fais cette répartition non propor- 
tionnellement aux contenances des forêts - ce qui est une base areugle, - non par rapport à leur produit en argent - ce qui serait une fausse manœurre consistant à donner le plus à la forèt qui en a le moins besoin, - mais en raison du produit moyen de l'hectare en volume et du prix du mètre cube dans la forèt. Je suis certain de dépenser ainsi mes fonds là où ils seront employés de la manière la plus fructueuse.

( Permettez-moi aussi, nous disait en terminant M. de N..., de vous faire remarquer avec quelle promptitude je puis répondre à toutes les questions de statistique et d'administration qu'il vous plairait de me poser. Combien les forèts produisent-elles de mètres cubes? Quel est le prix moyen du mètre cube sur tel ou tel point? Pour combien, dans ce prix, entre-t-il de produits principaux et accessoires? A combien s'élèvent dans chaque forèt les travaux d'amélioration de premier établissement faits depuis telle époque? Combien coùte leur entretien? Je pourrais répondre à toutes ces questions si importantes pour un administrateur en un instant, et si j'avais vingt ou trente fois plus de forèts à gérer que je n'en ai, je serais toujour's aussi prêt à répondre; ce ne serait qu'une affaire de centralisation de vingt ou trente documents fort simples. »)

Nous ne pouvions que reconnaitre les rẻsultals 
obtenus par M. le duc de N... et le remercier de ses obligeantes communications. Il y a dans la manière dont il gère les domaines forestiers de sa famille tout un systeme d'administration que nous livrons aux ètudes du public forestier.

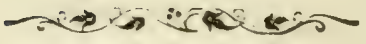




\section{TABLE DES TERUIS FORESTIERS.}

\begin{tabular}{|c|c|c|c|}
\hline & $\mathrm{H}$ & & Page. \\
\hline - & 18 & normale. . . . & \\
\hline - & 77 & lière ....... & \\
\hline ménagemt ${ }^{t}$. & 49 & leine ..... & 政 \\
\hline de taillis en & 129 & & 156 \\
\hline Coup & 75 & Par & 38 \\
\hline - & 75 & Péri & 31 \\
\hline$\ldots$ & 75 & tion. . . & 78 \\
\hline & 75 & $\ldots \ldots$ & 167 \\
\hline- & 87 & exploi- & \\
\hline t ... & 83 & $\cdots$ & 80 \\
\hline rcie ...... & 76 & l d'exploita- & \\
\hline rcie prépara- & & $\ldots \ldots$ & \\
\hline$\ldots \ldots$ & $8 \pi$ & $\operatorname{Prc}$ & 181 \\
\hline aircie de con- & & acipaux . . & 75 \\
\hline & & ires & \\
\hline & & - & \\
\hline- & 124 & $\begin{array}{l}\cdots \\
\cdots\end{array}$ & $\begin{array}{l}1 ! 2 \\
110\end{array}$ \\
\hline & 86 & aires ... & 110 \\
\hline ge & 160 & ve ..... & 113 \\
\hline & 151 & $\begin{array}{c}\text { - accidentels ou im- } \\
\text { prérus . . . . . . }\end{array}$ & \\
\hline 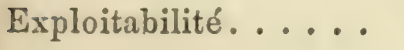 & & - accessolres. . . . . . & 20 \\
\hline
\end{tabular}


214 TABLE DES TERMES FORESTIERS.

Rapport soutenu .... Page. 181 Révolution de converReconduction en futaie. 118 sion . . . . . . 138 Règlement provisoire d'exploitation. . . . 61 - des éclaircies .... 87 Réserve ....... 51 Rotation des éclaircies. 87 - ̀̀ assiette fixe.... 52

- mobile ........53 Revenu. . . . . . . 181 - composé ou sous fuRévolution...... 31 taie......... 64 


\section{TABLE DES MATIËRES.}

\section{Économio forestièro.}

§ 10r. De l'Exploitation des furêts en général.

Capital d'exploitation................ 1

Production ......................... 6

Reute.......................... 8

Rôle des forêts de l'État. . . . . . . . . . 12

§2. De l'Aménagement en général.

Définition ....................... 18

Anciennes méthodes ..................... 19

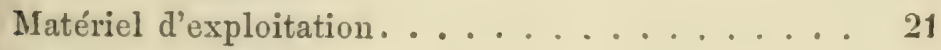

Explication de la définition .......... 29

Utilité des aménagements ........... 34

État des forêts .............. 36

Parcellaire ...................... 38

\section{Aménagement des forèts à petit capital d'exploitanion.}

TAILIJIS SIMLILES.

$\S 1 \mathrm{er}$. Taillis dont le Matériel d'erploitation est constitué.

A. D'une manière régulière.

Plan général d'exploitation ........... 46

Compte daménagement ............... 49

De la réserve...................... 51

Réserve ì assiette fixe............... 52

Réserve mobile............... 53 
B. D'une manière irrégulière.

Leur régularisation.

$\S 2$. Taillis dont le matériel d'exploitation est surabondant. Exemple.

§3. Taillis dont le matériel d'exploitation est insuffisant. Exemple.

TAILLIS COMPOSÉS.

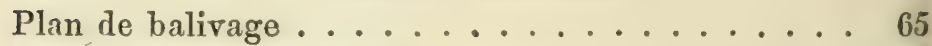

Compte d'aménagement.............. 67

\section{Aménagement des forêts à gros capital d'exploitation.}

FUTAIES PIEINES.

$\S 1 \mathrm{er}$. Futaies dont le matériel d'exploitation est constitué.

A. Régulièrement disposé.

Deux natures de produits . . . . . . . . . 74

Affectations. .................. 77

Plan général d'exploitation . . . . . . . . 80

Plan spécial d'exploitation. . . . . . . . . . 83

Produits principaux ................ 84

Produits intermédiaires : deux méthodes . . . . . 86

De la réserve................... . . . 95

Compte d'aménagement . . . . . . . . . 97

B. Irrégulièrement disposé.

Séries d'exploitation . . . . . . . . . . 100

§ 2. Futaies dont le matériel d'exploitation est surabondant. Considérations générales ... . . . . . . . . 102 Aménagement. . . . . . . . . . . . 103

Premier exemple................ 107

Du volume extraordinaire ............ 110

Compte d'aménagement . . . . . . . . . . . 114

Deuxième exemple. . . . . . . . . . . . 115 
§3. Futaies dont le matériel d'exploitation n'est pas constitué. Généralités . . . . . . . . . . . . . . 116

A. Reconductions en futaie pleine.

Premier exemple.

Deuxième exemple.

B. Conversions de taillis en futaie.

Considérations générales.

$\S 1 \mathrm{er}$. Taillis sous futaie réguliers.

10 Méthode des coupes préparatoires. . . . . . . 135

20 Méthode par le taillis temporaire. . . . . . . . 138

30 Méthode mixte . . . . . . . . . . . . 144

De la réserve. . . . . . . . . . . . . 151

§ 2. Taillis irréguliers.

Exemple . . . . . . . . . . . . . 154 FUTAIES JARDINÉES.

Généralités . . . . . . . . . . . . 156

De la possibilité . . . . . . . . . . . . 161

Amenagement. . . . . . . . . . . . . 167

De la réserve. . . . . . . . . . . . . 169

Exemple ................... 170

Compte d'aménagement............ 173

Comparaison avec la futaie pleine . . . . . . 174

Détermination de la possibilité d'une forêt.

Définitions . . . . . . . . . . . . . . 179

Énoncé d'un 1er problème. . . . . . . . . . 181

Son utilité . . . . . . . . . . . . 184

Solution. . . . . . . . . . . . . 186

Second problème. . . . . . . . . . 190

\section{Appendice.}

Gestion d'un ensemひle de jorêt . . . . . . . . . . . 193 
Modèles de registres et d'états . . . . . . . . 198 Aménagement . . . . . . . . . . . . . 198 Comptabilité . . . . . . . . . . . . . . 202 Comptes de gestion. . . . . . . . . . . . . 204 Résultats obtenus. . . . . . . . . . . . . . 208 Table des termes forestiers ........... 213

พื1 


\section{ROTHSGHILD, Editeur, 13, Rue des Saints-Pères, Paris}

\section{GHASSE - BOIS - FOREATS}

\section{Reboisement et Gazonnement des Montagnes Traité}

pratique, par P. DeMontzer, Inspecteur général des Foréts. - 20 édition, revue et augmentée. Un fort volume in-8 de 600 pages, imprimé sur

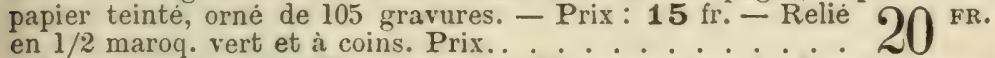

Les principaux Chapitres de l'Ouvrage sont: Description et formation des torrents. - Travail des torrents dans la montagne. - Constitution des Périmètres. - Corrections des torrents à affoulllements. - Exécution des travaux. - Torrents à clappes et torrents glaciaires. - Du reboisement en général. - Du choix des essences. Mesures et travaux préparatoires. - Exécution des travaux de reboisement. - Travaux de gazonnement. - Operations topographiques. - Travaux de voirie. - Hydrographie. - Entretien des travaux de correction. - Entretien des trávaux de reboisement. - Ordre chronologique des travaux à partir du décret d'utilité publiuue. - Travaux de reboisement et de gazonnements facultatifs.

'Art de Planter Traité pratique sur l'art d'èlever en pépinière et fruitiers et d'agrment de planter à demeure tous les arbres forestiers.

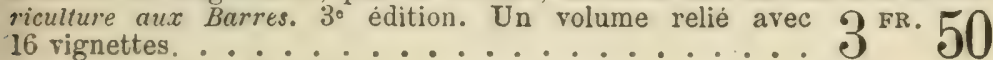
lanuel de Cubage et d'estimation des bois en futaies, taillis, lanuel de Cubage arbres abattus ou sur pied. Notions pratiques sur le debit, la vente et la fabrication de tous les produits des forêts, tarif de cubage des bois en grume ou équarris. Table de conversion, par

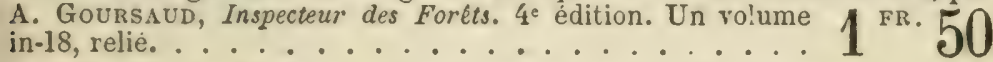

es Bois indigènes et étrangers Physiologie, culture, dustrie, commerce, statistique, etc., par MM. A. E. Dupont, Ingénieur des constructions navales, et BOUQUET DE LA GRYE, Conservateur res Forkls. - Un volume in-8, avec 162 gravures.

$12^{\mathrm{FR} .}$ es Bois employés dans l'Industrie Caractères distinctis, pagnées de 100 sections en lames minces des principales essences forestières de la France et de l'Algérie, par H. Nórdurisger, Professeur. 100 échantillons de bois, montés sur papier, avec textedescriptif 30 Fr.

es Conifères Traité pratique des arbres verts ou résineux, indigènes Foréts. Introduction par M. le Vicomte de Courval. Deux volumes, 5 Fr. reliès, avec 106 gravurss. Prix.

lans les Bois Quatrieme édition française, imitee sur la trentième édition allemande, par Lours ENAult, ornée de gralvures originales, dont plusieurs de page entière. Un volume petit in- $q^{\circ}$, imprimé sur papier teinté. Broché, 4 fr.; relié, 6 fr. Edition sur 8 Fr. papier de Hollande. 


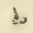

\section{REBOISEMENT}

E $\mathbf{T}$

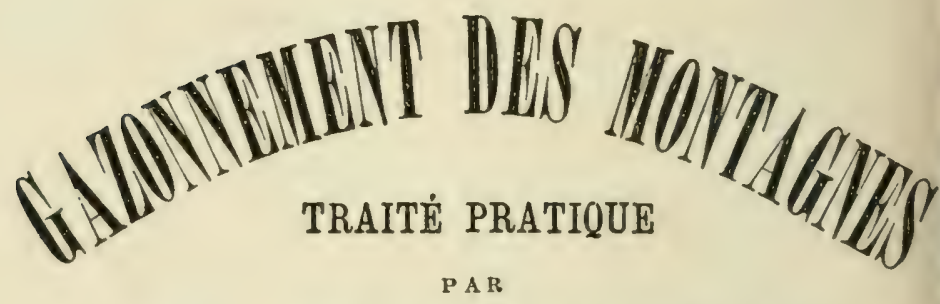

P. DEMONTZEY

Conservatour des Forôts

Ouvrage publié sous les Auspices

des Ministères de l'Agriculture, du Commerce et des Travaux Publics

\section{DEUXIÈME ÉDITION REVUE ET AUGMENTÉE}

Un fort rolume in $8^{\circ}$ de 600 pages, Imprimé sur papier teinté, orni de 105 gravures. Prix .......... $15 \mathrm{fr}$.

La prenière édition n'est pas venue dans le commerce Cet ouvrage, qui intéresse les Forestiers, Ingénieurs, Propriétaires, etc.: contient les chapitres suivants .

Description et formation des torrents. - Travail des torrents dans $l_{1}$ sein de la montagne. - Constitution des pèrimètres. - Correctior des torrents à affouillements. - Exẻcution des travaux. - Torrent à clappes et torrents glaciaires. - Du reboisement en général. Du chorx des essences. - Mesures et travaux préparatoires. Exécution des travaux d reboisement. - Travaux de gazonne ment. - Opérations topographiques. - Travaux de vorrie. Hydrographic. - Entretien des travaux de correction. - Entreties des travaux de reboisement. - Ordre chronologrique des travau: à partir du decret d'utilité publique. - Travaux de reboisemen et de gazonnement facultatifs. - Subventions et primes pou $\exists$ l'amélioration des pâturages.

CODE FORESTIER. - Recueil des lois, décrets, ordonnanc's avis du Conseil d'Etat et règlements en matière de forêts, chasse louveterie, dunes et reboisement, par A. Puton, Directeur el Pr" fesseur de Droil à l'Ecole nationale forestière. Un volume in-lई Prix.

$3 \mathrm{fr} .51$ 


\section{ROTHSGHILD, Editeur, 13, Rue des Saints-Pères, Paris}

\section{GHASSE - BOIS - FORETS}

\section{Nouveau Carnet de Chasse illustrẻ et augmenté du Manuel à manière de le conduire, moren de devenir bon tireur, conseils à un jeune chasseur, par M. Chatix. - 3e édition, ornée 1 FR. de 37 vignettes, reliè.}

es Animaux des Forêts Mammiferes et oiseaux. - Zoologie

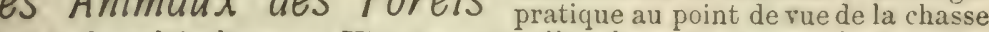
et de la sylviculture. - Histoire naturelle, chasse à courre, chasse à tir, entretien, conservation, reproduction. - 2 e edition par R. CABırrus, souvinspecteur des Foréts. - Un volume avec Śt gravures, 2 FR. 50

es Oiseaux-Gibier Leur description, chasse, mours, acclimaes OiseaUX-Gibier tation, par H. DE LA BLANchERE. Un volume in-folio, avec 45 chromotypographies et de nombreuses vi- 60 FR. gnettes, 50 fr.; relié.

Guide du Chasseur devant la Loi Recueil des lois, ordonministérielles avec les dispositifs, par ordre alphabétique, de toutes les décisions rendues en matiere de chasse, depuis le 3 mai 1815 jusqu'à ce jour, par F. TECHEsEY. - Un volume in-18, relié.

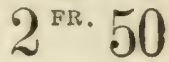

es Ravageurs des Forêts et des Arbres d'alignement. des insectes, morens de les combattre et de restaurer les plantations, par H. DE LA BLAxichERE et par EUG. ROBERT, Inspecteur des plartations de Paris. $-5^{e}$ édition. Un volume arec 162 gravures.

Prix, relié.

$3^{\mathrm{FR} \text {. }}$

50

Code de la Législation forestière. Recueil des lois, décrets, règlements en matiere de forêts, chasse, loureterie, dunes et reboisement,

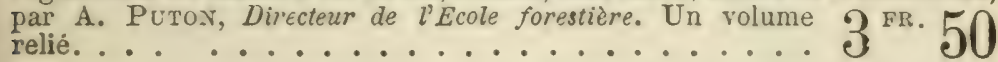

La Sy/viculture Résumé complet des règles de la culture des forêts. vateur des Foréts, 6 e edition. Un volume avec 70 gravures. 2 FR. 50

l'Aménagement des Forêts Etudes pratiques, par L. TAssr,

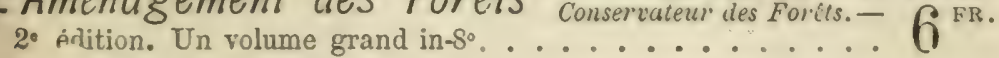

L'Aménageinent des Forêts Traité pratique de la conduite

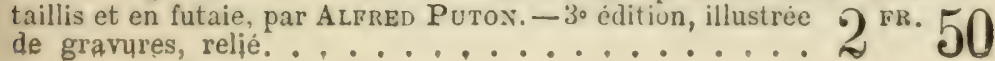


J. RoTHSCHILD, Editeur, 13, Rue des Saint-Pères, Paris. HISTOIRE NATURELLE - CHASSE A COURRE
CHASSE A TIR - ENTRETIEN - CONSERVATION - REPRODUCTION

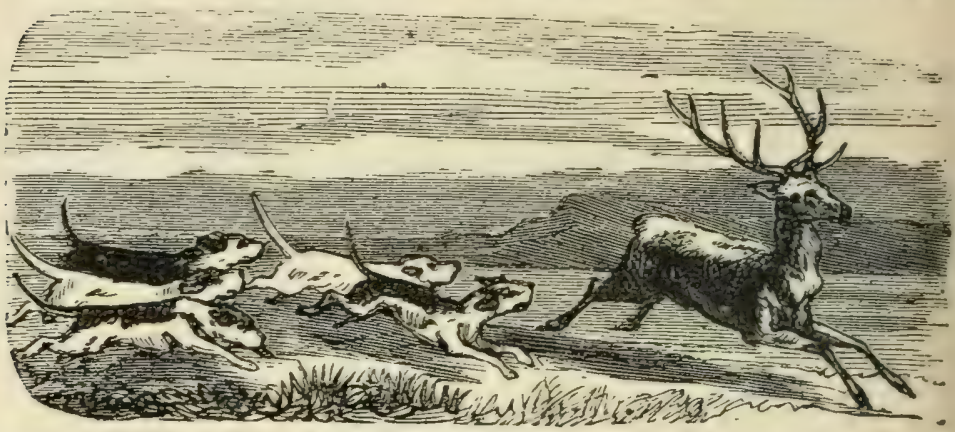

LES ANIMAUX

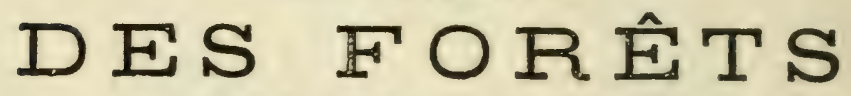

- MAMMIFÈRES - OISEAUX ZOOLOGIE PRATIQUE AU POINT DE VUE

\section{DE LA GHASSE ET DE LA SYLVIGULTURE}

A. L'USAGR DES CHASSEURS

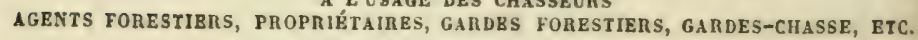

PAR R, CABARR U S

Sous-Inspecteur des Foréts de la Couronne

Attaché à la Vénerie de l'Empereur, ancien élève de l'École impériale forestière

1 vol. in-18, illustre de 84 vignetles sur bois, impression en caractères élzevirs a la maison Claye.

Broché (pour amatenrs) ou relié : 2 fr. 50
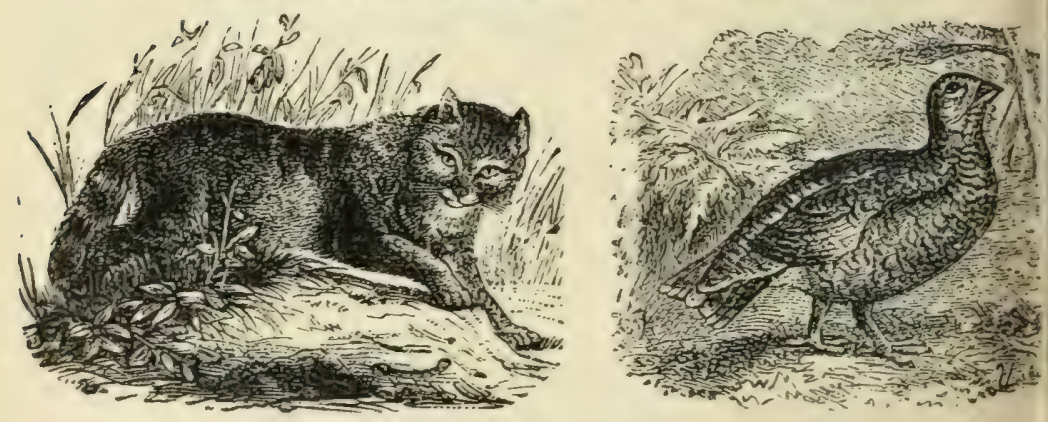


\section{J. ROTHSCHLD, Editeur, 13, Rue des Saints-Pères, Paris}

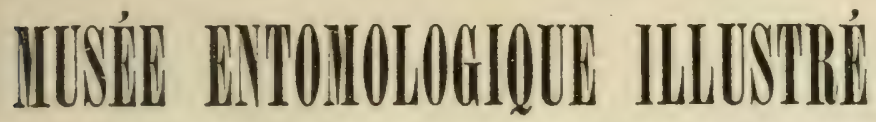

Histoire iconographique en 3 Volumes publiée par une Réunion d'Ento mologistes Français et Étrangers, avec 122 chromolithographies retouchées à la main, 1050 vignettes, représentant en couleur les Insectes, Chenilles, Chrysalides, Métamorphoses, les Plantes dont ils vivent, et leur organisation, mœurs, chasse et classitication.

\section{Chaque Volume se vend sépa'ément.}

Les Coléoptères - Description générale suivie d'une histoire Les Colépteres. naturelle des Coléoptères d'Europe. Un volume in $4^{\circ}$, avec 48 planches en couleur et 335 rignettes. Prix : $30 \mathrm{fr}$. Relie........................ 35 fr.

Les Papillons. - Description générale suivie d'une histoire A Depurset, 2e édition. et 260 viguettes. Prix : 30 fr. - Relié....... 35 fr.

Les Insectes - Histoire naturelle des Névroptères (Libel. (uptèr, Ephemères. Friganes, etc.); des Hymétères (Punaises, Cigales, Pucerons, etc.); des Diptères (Mouches, Cousins, Oestres, etc.); des Aptères (Lépismes, Podures, Puces, Pous). Un volume in $4^{\circ}$, orné de 21 planches en couleur et de 460 vignettes. Prix : $30 \mathrm{fr}$ - Reliè. ......... $35 \mathrm{fr}$.

L'œuvre, tout en étant savante, s'adresse à tout le monde. Elle a été du reste adoptée pour les bibliothèques des Ecoles et comme livre de prix par les Ministeres de l'Instruction publique de France et de Belgique.

Un Musée ne doit être fermè à personne. Celui-ci rappelle aux spécialistes, ce que déjà ils doivent savoir; il apprend aux Amants de la Nature, à la jeunesse studieuse, comment sonl organisés, comment vivent, comment on chasse, on collectionne et on classe les ordres, familles, tribus, genres des Insectes.

Dans une courte introduction, placée en tête du volume des Coléoptères, qui a paru le premier, les auteurs du Musée entomologique exposent avec précision, l'intérét et le caractère pariculier du sujet qu'ils ont traité.

L'Illustration que l'on prodigue volontiers aujourd hui, est ici particulièrement à sa place. Le texte, sur un pareil sujet, a besoin du crayon. Plus les planches et vignettes sont nombreuses, mieux on s'instruit, surtout lursqu'au nombre s'ajoute la qualité. C'est le cas 'pour le Musée entomulogique. Lies planches en couleur sont d'une très-bonne exécution, eiles contiennent surtuut notre faune; les vignettes sont a la fois exactes et jolies et figurent dans la description générale en tête de er que volume, en noir, les Insectes des pays hors d'Europe. On n'a rien épargné pour que l'œuvre, au point de vue de l'entomologie, fat complète et le but a été atteint. 


\section{J. ROTHSCHIL D, Editenr, 13, Rue des Saints-Pères, Parls.}

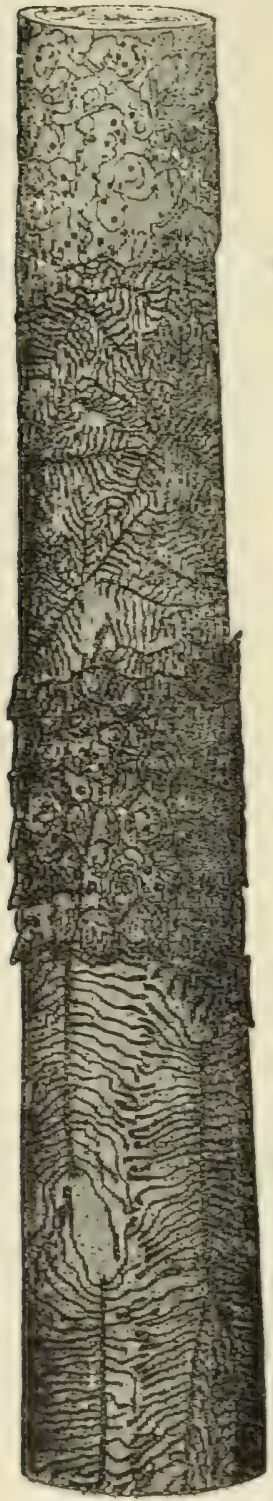

Vient de parattre la cinquième Ê.

LES RAVAGELRS DES FORÉTS

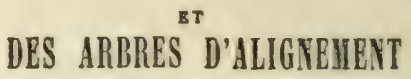



Histoire naturelle-Mœurs-Dégâts Moyens de Destruction

PAR

H. DE IA BLANCHERE Ancien élève de l'École forestière

BT

LE Dr EUGENE ROBERT

Un vol. in-18, avec 162 Grav,, reliẻ 3 fr. $5 \mathrm{C}$

Peu de publications ont eu autant de succès que cet ourrage dont la 5 e édition vient de paraître, revue et augmentée d'une centaine de figures. Nons ne pouvons mieux faire quo de citer l'opinion de M. P. Joigneaux:

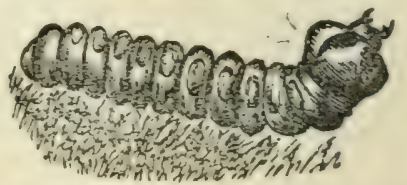

Tulgariser et intéresser en instmisant, tello est la devise du livre. Il renferme tout ce qu'il importe de savoir sur tous les Insectes Rava. geurs des Arbres forestiers, des Plantations des Pares et d'Alignement:

Les Arbres et les parties qu'ils attaquent: le mois d'apparition; l'état de l'Insecte lorsqu'il cause ses dégâts; le moyen de le combattre et de restaurer les arbres.

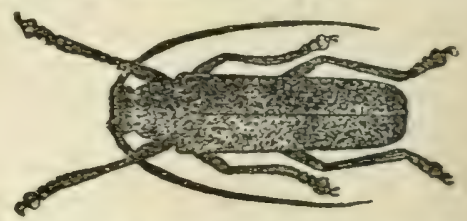

L'exécution matérielle du volume est extrêmecuent soignée; les nombreuses gravures ajouteut un intérêt spécial par la représentation des principaux acteurs des d:smes oylvaijas et de leurs travaux. 
J. ROTHSCHILD, Editeur, 13, Rue des Saints-Pères, Parıs

VIENT DE PARAITRE LA 20 ELITYON

Entièrement revue, augmentée de 50 pages de texte et de 150 gravures

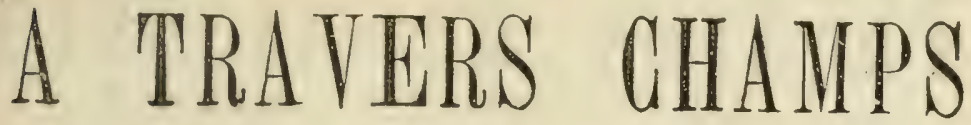

\section{BOTANIQUE POUR TOUS}

HISTOIRE DES PRINCIPALES FAMILLES VÉGÉTALES

\section{$M^{\text {me }}$ LE BRETON}

Un volume in-8, 550 pages, ornèes de 746 gravures sur Bois

Ouvrage de luxe, imprimé avec Caractères elzéviriens sur papier teinté; prix : 7 Francs. - Reliure spéciale en toile grise, tranches rouges, plat orné d'un dessin en couleur et à biseaux, 10 Francs.

Cegracieux livre, conçu dans un esprit tout nouveau, et qui se recommande autant par l'a. grément de la forme que par l'exactitude scientifique la plus scrupuleuse (il a été revu par II. Decaisne, le savant Professeur du Mrseum) ¿ été adopté par le Ministère de l'Instruction publique pour les Biblio. theques scolaires ct comme Livre de prix pour les

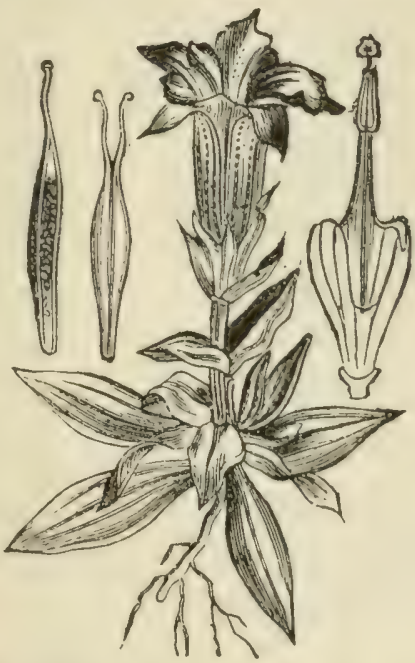

Écoles de la Ville de Paris.

L'auteur fait connaître à la jeunesse l'his toire des plantes, leur organisation, leurs fonctions, leurs propriétés, leur classification, les différents aspects qu'elles donnent à la terre et l'en. semble de leur rôle si varié dans le monde: le tout orné de magnifiques gravures.

Apprendre a aimer les plan. tes, qui par leur

charme et leur utilité se mûlent nécessairement it notre vie, et faire naître ainsi le désir de les mieux connaître, tel est le but de eet ourrage. 
J. ROTHSCHILD, Editenr, 13, Rue des Saints-Pères, Paris

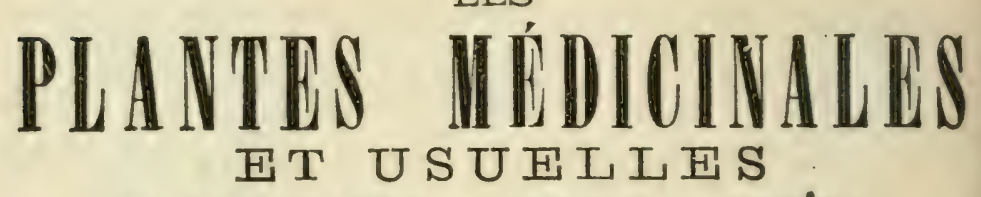

DE NOS CHAMPS - JARDINS - FORÊTS

DESCRIPTIONS ET USAGES

des Plantes comestibles - suspectes - vénéneuses - emplojéea dans la Médecino, dans l'Industrie et l'Économio domestiquo

\section{Par. H. IRODIN}

Membre de la Société botanique de France, lauréat, etc.

5. Édition. - Un volume de 500 pages avec 200 Gravures

Prix : relié, 4 Fr.

L'uuvrage que nous offrons au public comble une véritable lacune. Il s'arliesse aux gens du monde, aux jeunes gens, au clergé, aux habitants des campagnes, aux forestiers, aux éludiants; en même temps

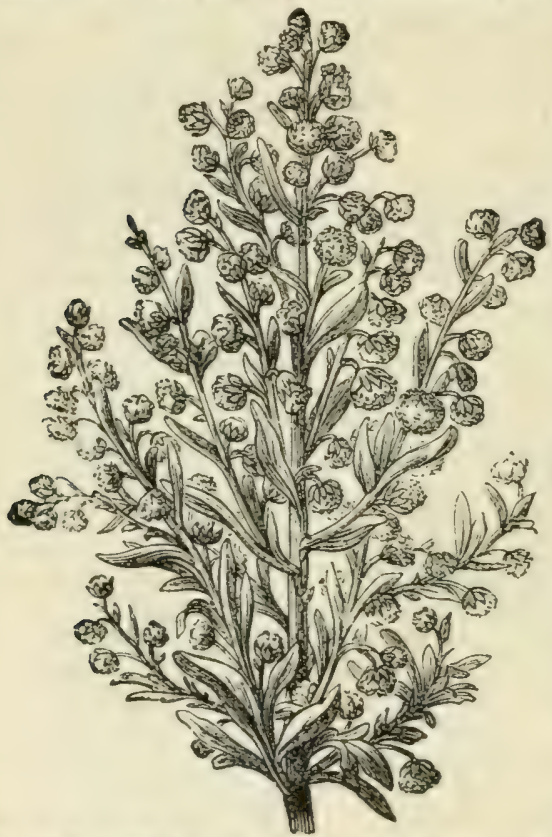
qu'il sera consulté avec fruit par les botanistes, les herboristes, les pharmaciens et le: " édecins; à la portée de tous, par la simplicite des expressions, par la clarté des descriptions, il trouvera sa place au foyer de toutes les familles. Les principaux chapitres sont :

Étude des simples. - Ré colte et conservation. Propriétés générales des familles. - Principes extraits des végétaux. - Stations des plantes médicinales. Les plantes émollientes, tempérantes; stimulantes. Toniques amères. - To. niques astringentes, antihystériques, altérantes, antispasmodiques, purgatives, etc., etc. - Utilite et cul. ture des plantes médicinales au point de rue forestier. Ies fulsifications.

L'ouvrage est accompa. gné d'une Table alphabé. tique des noms des plantes of a's fimilles, noms latins, français et vulgaires; d'une Table des maladies, remèdes, préparations; d'une Table des produits et usages. 
J. ROTHSCHILD, Editeur, 13, Rue des Saints-Pères, Paris.

VIEXT DE PARAITRE LA QUATRIËME ÉDITIOY

\section{LES CHAMPIGNONS}

Histure desuription, cultures, usages des especes comestibles, suspectes. vineraeuses et emplojees dans les arts, daas l'industrie, l'économie domestique et dans la médecine

\section{PAR F.-S. COIRDIER}

Doiteur en Médecine, Membre de plusieurs Sociétés sarantes

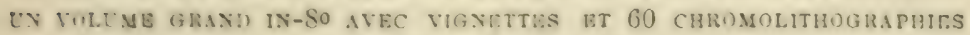
Contenant 30 J Sujets

Dessins originaux d'après nature par A.-E. Cordier

Prix broclue : $30 \mathrm{Fr}$; en demi-reliure chagrin, plats toile, tranches dorèes : 35 Franes.

Litude des clampignons ost grinéralement négligée, les livres étant trop scientiliques on dinn prix trop éleré. Nous avons évitéces écueils, et, pour bien faire apprécier la variété des sujets que l'auteur a traités

dans cette publication, nous énumérons les titres desprincipauxchapitres:

DIVISION DE LA PREMIẼE PAnTIE: De l'organisation; - de l'influence de la saison, du climat, du sol, de l'habitat, de la culture; - des moyens de distinguer la composition chimique; - de la possibilité d'enlever le principe Deseription de tous les champignons, avec leurs figures en chromolithorraplie, - glossare; - bibliographie; - table des noms vulgaires; - tahle alphahétique de tous les noms latins, français et rulgaires. cités dans l'ourrage.

(7) Cotte belle publication s'adresse aux lontanistes, aux chimistes, aux Bibliotheques publiques, aux industricls, aux médecins et à tous ceux qui trouvent goùt à létude de la nature. tion ; - l'emploi alimentaire; - la préparation culinaire; - l'eflet des champignons vénéneux; - des symptomes de l'empoisonnement ; moyens de remédier aux accidents. Contexu De I.A DEUNIEMP PARTIE :

toxique;l'emploi, la récolte, la culture; - la culture de la truffe ; moyeus de conserva - 


\section{J. ROTHSCHILD, Ėditeur, 13, Rue des Saint-Pères, Paris.}

\section{LES OISEAUX UTILES}

\section{ET \\ LES OISEAUX NUISIBLES}

AUX FORÊTS, AUX CHAMPS, AUX JARDINS, AUX VIGNES, ETC. Utilité, Ravages, Mœurs et Classement populaire PAR H. DE LA BLANCHERE Lu beau volume in-18 de 400 Pagres arec 150 Grarures sur Bis, Rralie : : fr. 50 DEUXIÈME ÉDITION REVUE ET ALGMENTÉE

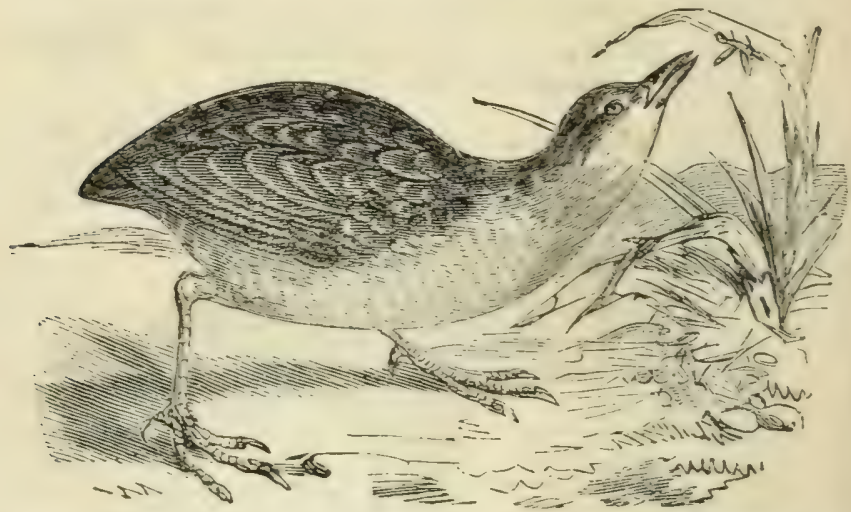

Aujourd'hui que l'on se préoccupe de la disparition croissante de ces utiles a xiliaires, et des moyens d'y remédier, ce livre est indispensable a tous: Hommes de ville ou de campagne, cultivateurs, forestiers, vignerons, jardiniers, etc.

Pour mieux taire apprécier le contenu de ce joli volume, nous citons le classement populaire que l'auteur a adopté :

CISEAUX DES BOIS Chap. Ier. - Habitants des grands massifs.

- II. - Habitants des lisières.

- III. - Eplucheurs de trones.

\section{OISEAUX DES CHAMPS}

Chap. ]V.-Habitants des haies et es huissons.

-. V. - Hôtes des sillons et des plaines.

- VI. Chasseurs dinsectes au vol.
OISEAUX DES JARDINS Chap. VII.-. Mangeurs de fruits. - VIII. - Voleurs de graines.

- IX. - Chercheurs d'insectes.

- X. - Chasseurs de nuit. OISEAUX DES RIVIËRES Chap. XI.-Oiseaux de marais. - XII._Oiseaux des rivages.

- XIII. - Oiseaux des grande: eaux.

OISEAUX DES VIGNES Chap.XIV. - Mangeurs de rai.ins - XV.-Mangeurs d'insecte: 


\section{J. ROTHSGHILD, Éditeur, 13, Rue des Saints-Pères, Paris}

\section{MINERALOGIE - GÉOLOGIE PALEONTOLOGIE}

Traité de Paléontologie Description et figure des animaux et tologiques en France; moyen pour extraire et préparer les iossiles, par Stanislas Megrier, Aide naturuliste au Musémm. Un trés-fort volume avec cartes en couleurs, illustré d'environ 1,000 rignettes.
Prix relie, environ

La Terre Son histoire. Etude de ses transformations successives. in-18 cartonné toile.

Géologie technologique traité de ses applications aux Arts et DrPage, traduit et augmenté par Stanislas Meunier, Aide- 3 Fr. naturuliste au Muséum. Un vol, avec 80 gravures. Relie en toile. 3 JU

Les Roches Guide pratique pour leur détermination, arec les conEdound Jannetraz, dide de minéralogie au Aruseum. - $2^{\circ}$ édition. Un volume avec plusieurs centaines de gravures et 8 planches en 5 Fr. couleurs; relie en toile. Prix environ ......... J

Le Chalumeau Analyses qualitatives et quantitatives. Traduction

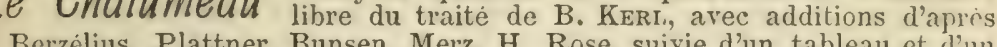
Berzelius, Plattner, Bunsen, Merz, H. Rose, suivie d'un tableau et d'un appendice spécial pour les applications minéralogiques, par EdocakD

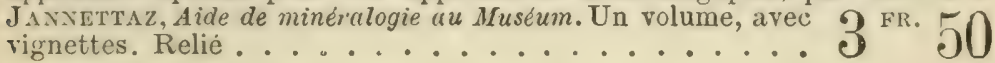

Les Minéraux Guicle pratique pour leur détermination sùre et rapide au moyen de simples recherches chimiques par voie sèche et par voie humide. Traduit d'après KoBeld, par le Comte L. DE LA TOUR DU PIN, arec nombreuses addilions par F. PISANI, Professeur de chimie et de minéralogie. - 30 èdition. Reliè. Prix

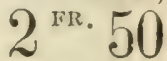

Diamant et Pierres précieuses Descriptions, Gisements, Emploi artistique et industriel, Evaluation. Statistique, Commerce des pierres précieuses, du corail et des perles. Avee une monographie historique des Bijoux, Joyaux et Orfevrerie. Ouvrage in-So, avec 350 gravures et une planche en chromo, par MM. ED. Jaxvetraz (meîlve des conférences de la Surbonne); FunLe Vandenherar (expert en diamunt); Eucibat: Fontenay (bijoutier-joaillier) et A. CouTANCE (professeut aux 20 Fr. Ecoles de la murine).

Reliẻ 1/2 maroquin, avec fers. - 25 Fir.

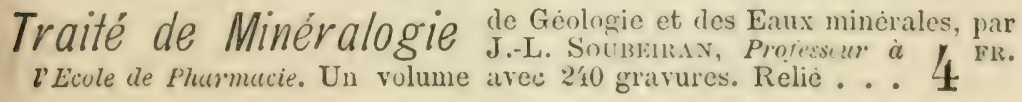


J. R0THSGHILD, Editeur, 13, Rue des Saints-Pères, Paris

Causeries scientifiques Découvertes et Inventions, progrès de DE PARVILLE. - 22 années sont en rente.

Les Tomes $S$ à $13,16,17,19$ à 22. Prix de chaque rolume. $3^{\text {FR. }} 50$ Le Tome 1s, contenant l'Exposition. Prix broché 5 fr.; relié

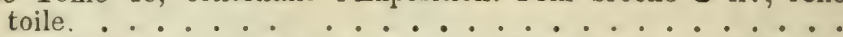

$6^{\mathrm{Fr} .}$

Il nous reste encore quelques exemplaires des tomes 7, 14 et 15 à 15 fr. chaque.

Les Tomes 19 à 22 viennent de paraitre.

Les Phénomènes de l'Atmosphère $\begin{gathered}\text { Traité illustré de Mé. } \\ \text { teorologie pratigue. }\end{gathered}$ Traduit et annoté par DECAUdix-LABESSE, d'après le professeur MoHs, Directeur de l'obserzatoire météorologique de Norriège. Augmentée d'une introduction par HexRI DE PARville.-Ouvrage illustré de 200 gravures, et de 24 cartes en couleur. Un volume grand in- $\delta^{\circ}$, imprimé avec luxe sur papier teinté. Prix 7 fr.; relié.

$10 \mathrm{FR}$.

Le Monde sidéral Description des phénomènes célestes, d'après
Zurcher récentes découvertes de l'astronomie, par
Prix. Margolté. Un volume avec 66 vignettes. 3 Fr. 50

Le Microscope Théorie et applications; traité illustré traduit

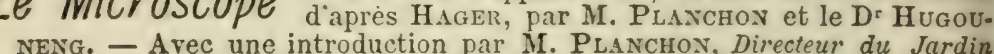
botaniqae de Montpellier Un volume ornè de 350 vignettes. $4^{\mathrm{FR}}$

Il contient tout ce qui peut intéresser l'Agriculture, l'Industrie, la Médecine et la Vie journalière; fermentations, maladips. aliments, tissus, bois : tout s'y trouve figuré el décrit.

Grand Atlas universel contenant 51 cartes gẻographiques sur d'après les explorations les plus récentes et les documents les plus authentiques, par Williax Hughes, Membre de la Société royale de géographie de Londres. Edition précédee d'une introduction par E. CortasBERT, Conservateur à la Bibliolhèzue nationale, avec Indrx général de tous les noms qui sont dans l'Atlas. Un wol. in-folio avec 51 cartes $1000^{\mathrm{FR}}$.
grarées sur acier et imprimées en coulcur. Reliè en toile. .

Relié en demi-maroquin.

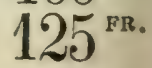

Le Télégraphe terrestre, sous-marin et pneumatique. Histoire, tarifs, etc., par PAUL LaUrescin. Un rol. aree 150 grar. Reliè.

$3^{\text {re. }} 50$

La Terre et ses Peuples Traité de géographie pittoresque, Hellwald, par Ed. Goepp (Chef au Ministère de l'Instruction publique). Un fort volume in- $\$^{\circ}$, orné de $\$ 00$ gravures dont plusieure ventaines spécialement dessinees pour l'édition française. Elle paraitra en livraisons $\dot{a}$.

》.50 


\section{J. ROTHSCHILD, Éditeur, 13, Rue des Saints-Pères, Paris}

L'Homme Développement phrsique et intellectuel de l'homme, par le ornè de 87 gravures.

Les Sociétés Leurs origines et leurs développements, par le 480 pages.

Les Enfants Education, instruction. Ce qu"il importe de faire saroir Les Enfants aux hommes, aux femmes, par Chinprerury. 4e édition de luxe. Un volume petit in $4^{\circ}$, arec planches en couleur, $10^{\mathrm{Fr}}$ eaux-fortes, et 70 gravures. Prix : 7 fr. 50 ; relie.

La Chirurgie du Foyer Traite populaire contenant: Inflamma. virulentes, tumeurs, empoisonnements, asphyxie, eli., etc., par le $\mathrm{D}^{\mathrm{r}} \mathrm{CI}$. BABAULT, Ancien Interne des hôpitaux. Un volume in-1S avec 3 Fr. 45 gravures, relie en toile. .

$3^{\mathrm{Fr} .} 50$

Le Médecin des Enfants HJgiène et maladie. Guide des mères Bock, Ballard, et Bower Harrison. Par A. C. BAnTHELEMr, Docteur d'apres en Médecine. $3^{e}$ édition. 1 volume.

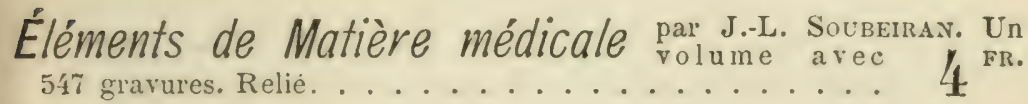

Les Aliments Guide pratique pour constater leur falsifications, d'après

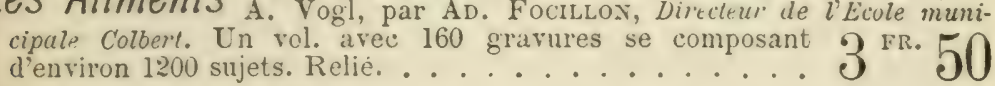

Les Souffrances du Professeur Delteil par CirnapprLevr.

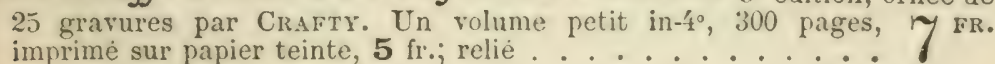

Ornithologie du Salon $\begin{aligned} & \text { Synonymie, description, mours et nour- } \\ & \text { riture des oiseaux de volières, europeens }\end{aligned}$ et exotiques, par R. Bovurart, Prépurateur au Mrusíum. Un volume grand in-S', arec 75 rignettes et 40 chromotypographies, repré- 35 Fr. sentant les oiseaux, leurs wufs et leurs nids, 30 ir.; reliè . . 32

Les Papillons de France Histoire naturelle, mours, chasse, Les Papillons de France préparation des Papillons, indications pour former des colle tions, etc. Un volume grand in-S', orne de 110 vignettes et accompagne d? 19 chromolithographies. 7 ás.; $1 \mathbf{C}^{\mathrm{PR}}$ rans une reliure de lus? 


\section{J. ROTHSCHILD, Editeur, 13, Rue des Saints-Pères, Paris}

\section{VIENT DE PARAITRE LA $11^{\circ}$ EDITION}

\section{REVTE ET AUGMEXTEE

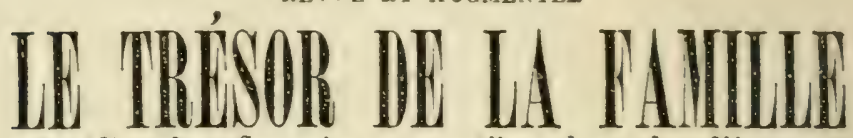

Encyclopédie des Counaissances utiles dans la Vie pratique d'après la 74e édition anglaise

\section{Traduite et modifiée pour la France}

\section{Par J. P. HOUZÉ}

Un fort volume de 900 pages, relié avec luxe, tranctes en couleur, $11^{\mathrm{e}}$ édition française, entierement revue et augmentée Prix. $5 \mathrm{Fr}$.

Cet ouvrage se propose la solntion de tous les problemes de la Vie pratique; il traite de toutes les connaissances utiles, propres a procurer le bien être et le bonheur domestiques. Il a pour but de mettre à la portée de chacun toutes les notions usuelles, tous les renseignements utiles dont on a besoin chaque jour. Il renferme tout ce qui concerne l'habitation, l'ameublement, l'alimentation, l'horticulture l'agriculture, l'habillement, la toilette, l'hygiene, la médecine et la pharmacie domestiques, l'éducation et l'instructiou des enfants, les usages de la socièté, les règles de la politesse, les lois de l'économie domestique et ces mille recettes d'une application facile et d'une si grande utilitẻ dans la vie.

Le Tresor de la Famille, fournit encore des renseignements précieux à un autre point de vue: en France ou nul n'est censé ignorer ja loi, il est bien peu de citoyens qui connaissent même les principaux points de la législation qui nous gouverne. Pour combler cette lacune, lauteur s'est efforcé de résumer les lois usuelles; les règlements de police et les connaissances necessaires pour permettre a tout citoyen intelligent de mener lui-même ses affaires a bonne fin.

Un travail aussi considerable, qui embrasse des sujets si divers, n'a pu être fait sans le concours d'hommes spéciaux, qui ont revu arec soin chaque partie. Ainsi les questions d'architecture ont été traitées par un architecte, la médecine par un médecin, la cuisine par un maitred'hôtel, la législation par un avocat, etc.

Plusieurs Ifinisteres et Administrations publiques ont bien roulu faire réviser les Parties concernant les postes, les télégraphes, les contributions, les lois militaires, le Timbre, etc.

Suivant le précepte d'Horace : ulile dulci, l'agréable est joint à l'utile, en donnant sur tous les jeur: jeux grmnastiques, jeux d'esprit, ieux de calcul et de hasard, recréations artistiques et scientifiques, tous les renseignements nécessaires.

En un mot, les auteurs se sont efforces de i'y rien omettre, afin que co livre soit rè-llement ce qu'il prétend êf : Une v ritable Encyclopédie des Choses usuelles. 


\section{J. ROTHSCHILD, Editeur, 13, Rue des Saints-Pères, Paris}

\section{BEAUX-ARTS - ARGHÉOLOGIE}

Paris Histoire, la ville, la voie publique, les promenades et monuments. Paris - La rie. - L'instruction publique. - Les arts. - L'industrie. - Le commerce. - Le dessous de Paris. - Les administrations. Paris à rol doisean, par MM. Jules Simox, A. Alphand, de Chexise-

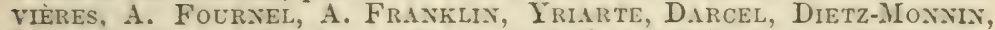
Leroy-Beaulieu, Claretie, Cherville, S.rrcer, Laugel, etc., etc. Les eaux-fortes, chromos et illustrations dans le texte sont par Mim. Didier, Dardoize, Alloxgé, Weber, Grandsire, Fraxgais, Diubigiy, Gejectte, Gilbert, Detaille, Giron, Rexouard, TourasFIEWICZ, Benoit, Delaunay, etc., etc.

Ouvrage de luxe dans le format in-folio, publié en livraisons et $5 \mathrm{FR}$. en sèries mensuelles à

Florence Histoire, la Renaissance, les hommes célèbres, la peinture, la sculpture et l'architecture, par CharLes YrIARTE. Un rolume in-folio de 400 pages arec 500 illustrations, dont 80 de page entière. Prix, en carton, $60 \mathrm{fr}$; ; reliè demi-maroquin arec fers spé- 200 FR. ciaux, $80 \mathrm{fr}$; ; edition sur chine.

Venise Histoire, commerce, architecture, sculpture, peinture, la ville, la vie, ete., par Charles Yriarte. Un volume infolio orné de 525 gravures. Prix, relie en demi-maroquin. . . 100 Fr.

\section{La Vie d'un Patricien de Venise au XVI siècle, d'après les papiers d'Etat des Archires des Frari.} ouvrage couronné par l'Académie française. - Edition de luxe illustrée de 136 grarures. dont 16 tirees hors texte et $\$$ planches sur cuirre diaprès

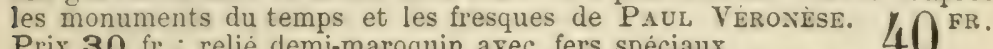
Prix $30 \mathrm{fr}$; relié demi-maroquin avec fers spéciaux. . . . 40

50 exemplaires sont imprimés sur Japon. Prix $60^{\mathrm{FR}}$

Rimini Histoire d'un Condottiere au $\mathrm{XVe}$ siècle. - Etudes sur les Rimini lettres et les arts a la cour des Malatesta. Un volume in- $8^{\circ}$, imprimé avec luxe en elzévir, arec 200 gravures d'après les monuments du temps. Prix : $25 \mathrm{fr}$; relie toile, $30 \mathrm{fr}$; relie en demi- 60 Fr.
Les Arts en Italie
Histoire des rilles, leur importance au point de vue de l'art antrefois et aujourd'hui, pein. ture, sculpture, architecture, mosaïque, ete. - Ouvrage de grand luxe, format de Florence et de Verise, publie avec le concours des meilleurs écrivains français, italiens, allemands, anglais et suisses, orné de 600 vignettes et 50 eaux-fortes.
(Sous presse.)

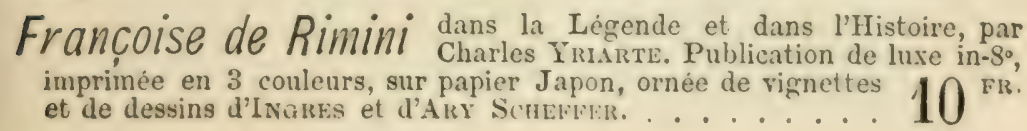




\section{J. R0THSGHILD, Editeur, 13, Rue des Saints-Père3, Paris}

\section{BEAUX-ARTS - ARGHÉOLOGIE}

Donatello à Padoue Monographie de ses chefs-d'œurre à Padoue: le Gatamelata et le Santo, par W. BoDE; traduction française revue et publiée sous la direction de Charles YRIARTE.

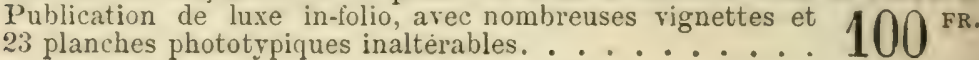
Les Médailleurs de la Renaissance par Alois Herss. Belle paraissant par monographies séparées; ont paru jusqu'à ce jour:

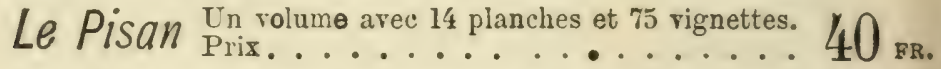

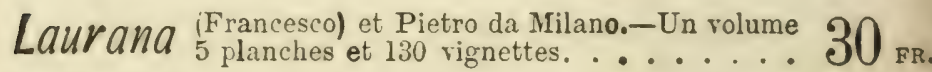

Este (Les Médailleurs de la Maison d'). - Marescotti, Lexignolo, Este Petricini, Baldasare Estence, médailleurs anonymes tra-

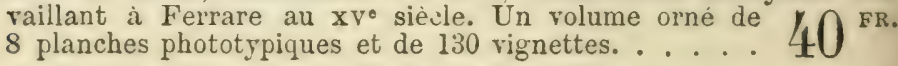

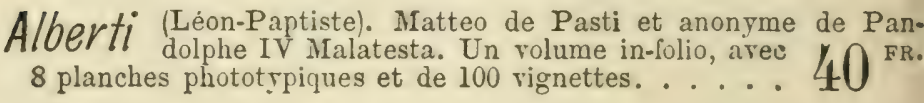

Les Médaillons de l'Empire romain Numismatique antique, guste jusqu'à Priscus Attale, par W. FRGENER. Ourrage de $45^{\text {FR' }}$ luxe in $4^{\circ}$, orné de 1,310 gravures. Prix $40 \mathrm{fr}$; ; relie . . . . 45

L'Art ornemental au Japon Dessin, Peinture, Gravure sur Tissus, Broderies, Laques, Emaus, Impressions en couleur, Incrustations, Sculptures sur iroire cloisonnes, Ourrages en metal, l'anglais et revu par M. Charles YrisRTE. - Ouvrage de grand luxe. - Les planches en chromo (tirées jusqu'en 24 couleurs), dépasseront tout ce qu'on a fait en couleur jusqu'à ce jour. Le texte, orné de quelques centaines de vignettes, reproduites d'après les originaux, est imprimé en plusieurs couleurs. Près de 200 vignettes et 100 planches de gardes japonaises feront l'ornement spécial de l'èdition française. Le tirage de l'édition française est fait à 400 exemplaires sur papjer blanc et i 100 exemplaires sur papier japonais de premier choix; tous les exemplaires seront numérotés à la presse. Prix de l'édition sur 600 FR.

Le Musée de Marine du Louvre. - Histoire, description, conset à voiles, par Edmond PARIs, Vice-amiral, Conservateur au Lourre.

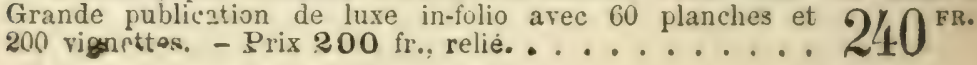




\section{J. ROTHSGHILD, Editeur, 13, Rue des Saints-Pères, Paris}

Les Chemins de Fer francais Etude historique sur la con- Deliats parlementaires. Actes législatifs, réglementaires, adminis. tratifs, etc., par AlrkFD PICARD, Cunseiller d'Elal, ancien Directeur des C'hemins de ler au Minislere des Travanx Publics.

Belle publication formant 4 volumes in $-8^{\circ}$, avec 2 cartes de la France et de l'Algèie en chromo. Prix bruché....... UU

OU FR. YORR.

Reliés en toile. Prix

Les Promenades de Paris Histoire, description des embellio d'entreticn des Bois de Boulogne et de Vincennes, des Champs-Elrsées, pares, squares, boulevards et des promenades intérieures de la ville de Paris, par A. AlPhAND, Inspecteur general des Ponls et Chaussées, Directeur des Travaux de la Ville de Paris.

Ourrage formant deux volumes in-folio, illustré de 80 grarures sur acier, de 23 chromolithographies et de 487 gravures sur bois . GीOFR. Prix 500 fr., relie.

L'Alimentation

du Canal de la Marne au Rhin et du Canal de l'Est, par AlFRED PIC.IRD. Cet ourrage traite des travaux exécutés depuis 1870 pour l'alimentation commune a ces deux roies narigables et pour l'alimentation spéciale à la première. - (Machines èlèvatoires hrdrauliques et à vapeur, réservoirs, rigoles. - Description et indications detaillées sur les dépenses de construction, d'entretien et d'exploitation). Un rolume de texte in-8 ${ }^{\circ}$ accompagné d'un fô. atlas de 25 planches

Les Travaux publics de la France. Ourrage de grand luxe, Trovaux publics et sous la direction de M. LEoxce REIxAU, Inspecteur général des Ponts et Chaussees, par MMI. les Inspecteurs généraux et Ingénieurs en chef des Ponts et Chaussèes: FElix Lucis, A. Fourie. Ed. Colligyon, H. de Lagrexe, E. Allard, Voisis-Ber. - Les ciny parties de l'ourrage contiennent: Routes et ponts, chemins de fer, rivières et canaux, ports de mer, phares et balises. Chaque partie forme un volume in-folio; elle contient 50 planches phototypiques et a sa carte spéciale. Prix.

$120^{\mathrm{ER}}$

L'ourrage complet formant 5 forts volumes grand in-folio (de 57 sur 42), avec 584 pages de texte et 538 vignettes, 7 cartes tirées en couleur, 250 planches faites d'après nature, est du prix de $600 \mathrm{fr}$; relié en 5 volumes, planches sur onglets toile, tranches dorées.

Yh $\mathrm{FR}$.

Les Alhes au point de rue de la Geographie physique et de la Géologie. CS Alpes - Voyages photographiques dans le Dauphiné, la Savoie, le nord de l'Italie, la Suisse et le Trrol; avec 14 héliogravures, d'après les Photographies de l'auteur, retonchees par JACQUET, et une carte an $1 / 600,000^{e}$, indiquant les courbes d'horizon des Panoramas, par A. Civiale. - Le prix de l'ourrage est de $50 \mathrm{fr}$. pour le volume de texte avec la carte des courbes d'horizon; il est de $65 \mathrm{fr}$. pour le volume de texte avec les deux cartes. 


\section{A GRIGULTURE, ETC.}

La Vigne dans le Bordelais. - Commerce, culture, etc.,par 12 Fr.

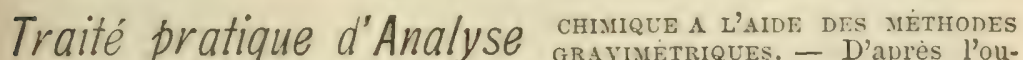

vrage de Thorpe, par Stavistas Mecrier, Aide naturaliste uu 5 FR. Muséum. Un volume arec 111 vignettes dans le texte. Relié .

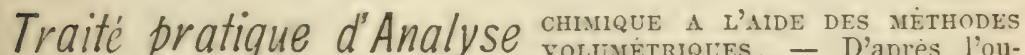
vrage de $F$. Sutton, par $\mathrm{Folumetrieces,} \mathrm{-} \mathrm{D'apres} \mathrm{l'ou-}$ avec 95 rignettes dans le texte, reliẻ ..........

Les deux Traités pris ensemble............... 8

15s La nécessilè de l'analyse chimique se fait d'autant plus sentir aujourd'hui, que toutes les transactions commerciales, achat et vente de matières premières ou manufucturées, reposent sur des analyses qui indiquent leur teneur en substances utilisables.

Le cultivateur lui-même, qui niaccepte les indications de la science quavec une extrime prudence, n'achète plus d'engrais sans les faire analyser.

Tout en traduisant deux livres extrémement répandus en Angleterre, les auteurs $y$ ont fait de nombreuses additions et se sont offorcés de les mettre au courant de l'industrie française.

Ces deux ouvrages se recommandent donc spécialement aux agriculteurs, aux. chimistes, aux ingénieurs, aux mélallurgistes, aux industriels, aux médécins, aux pharmaciens, qui $y$ trouveront exposés arec dé ails, l'analyse des divers produits de l'industrie, des minerais, des phosphates, drs guanos, des sucres, des eaux, des gaz, des terres, des urines, etc.

La Culture économique par l'emploi raisonné des instruments, usités dans la machines, outils, appareils, ustensiles un beau vol. in-1s de 350 pages, illustre de 20 figures. Relie. 2 50

La Terre végétale De quoi elle est snits romment elle se forme, géologie agricole, par Stanislas MEuxier.

une carte agricole de la France, par $\mathbf{A}$.

l'Ecole normale. Un volume relié.

\section{Anatomie et Physiologie de I}

tenant 12 lithographies et un texte ex

Gimnwori.

\section{Nos Champignons $\begin{gathered}\text { Leur descriptit } \\ \text { sentation des }\end{gathered}$}

vẻnéneuses, qu'on rencontre généralemer

J.-A. Barral. Un volume in-1 ${ }^{\circ}$ avec 16 ? 


\section{J. RoTHSCHILD, Ėditeur, 13, Rue des Saints-Pères, Paris}

\section{TRAITÉ PRATIQUE}

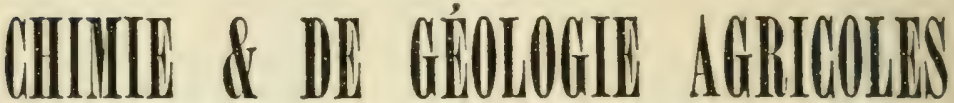

Traduction libre d'après la onzième édition anglaise des professeurs Johnston et Cameron

\section{PAIR STANISLAS MEUNIER}

A ide Naturaliste de G ologie au Musérm, Lauréal de l'Inslinut Ln volume in-18 de 400 pages, orné de 200 vignettes, relié toile. Prix.............. 3 Fr. 50

Cet ouvrage devrait se trouver entre les mains de tous les cultivateurs, qui ont un intérêt si manifeste à connaitre leur sol et à ètudier les moyens de les fertiliser, afin d'en tirer le meilleur profit possible.

Ils y apprendront quels sont les élements constitutifs des plantes, la comnosition et les propriétés physiques des sols, les moyens de les améliorer, les lois de l'assolement et de la croissance des vegétaux, le rôle des divers engrais et amendements sur la fertilite des terrains, leurs applications aux récoltes, les principes de la nutrition et de la production animale, etc.

Ce volume qui renferme un grand nombre de vignettes d'une exćution très-soignée, résume les plus importantes découvertes de la science et les presente sous une forme parfaitement intelligible pour les cultivateurs.

\section{Vient de paraitre le second Tirage DICTIONNAIRE VÉTÉRINAIRE}

Hygiène, Médecine, Pharmacie, Chirurgie, Mnltiplication, Perfectionnement des Animaux domestiques

PAR L. FÉLIZET

Avec une Introduction par J. A. BARRAL

Deuxième Tirage. - Un beau volume in-18 de 465 pages, relié toile....................... 3 Fr. 50

Le but de l'auteur est de guider les cultivateurs dans les premiers soins qu'ils doivent donner à leurs bestiaux en àttendant l'arrivée du vétérinaire.

Cet ouvrage est adopté en France et en Belgique, sur des rapports très-favorables, par le Minislére de l'Instruction pu'lique, et nous avons refait un nouveau tirage, afin de voir arriver ce joli volume dans toutes nos bibliothèques des campagnes, et entre les mains de tous

on 


\section{J. ROTHSGHLD, Ėditeur, 13, Rue des Saints-Pères, Paris}

\section{GEOLOGIE TECHNOLOGIQUE}

TRATT DES APPLCATIONS DE IA GÉOLOGIB

\section{AUX ARTS ET A L'INDUSTRIE}

Agriculture. - Architecture. - Génie civil. - Métallurgie.

Céramique. - Verrerie. - Médecine. - Produits chimiques. - Teinture.

Peinture. - Joaillerie.

TRADUCTION LIBRE DR L'ECONOMIC GEOLOGY, DR DAVID PAGE

Professeur de Géologle a l'Untrersité de Durham

\section{PAR STANISLAS MEUNIER}

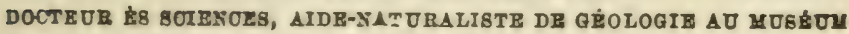

Un fort volume 1n-18, orné d'environ 80 grarures.

Rellé tolle anglalso. Prix : 3 fr. 50.
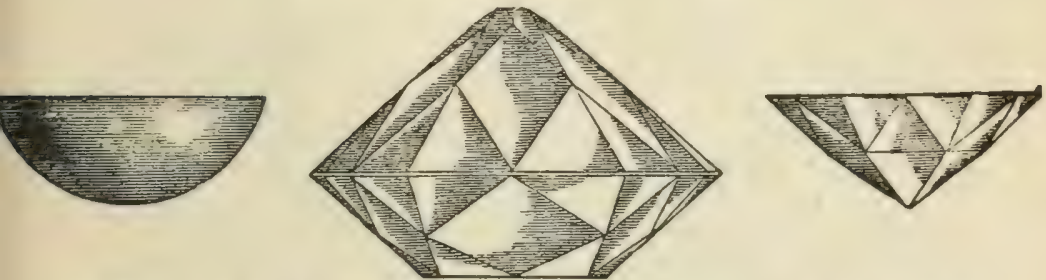

Se trouvant dans un centre à la fois métallurgique et manufacturier, l'auteur était à même d'écrire de visu sou Economic Geology. M. Stanislas Meunier s'est attachè à faire passer ce livre dans notre langue, en remplaçant par des localités françaises affrant les mêmes faits, tous les points étrangers signalés par l'auteur. Le but à attein. dre était de faire un tableau des richesses minérales du globe, ordonné d'après les diverses applications de chacune d'elles.

Les principales divisions du livre, illustré d'environ 80 figures, sont: Résumé des notions géologiques élémentaires offrant la description de la croute rocheuse, puis l'exposé des applications de la Géologie aux points de vue les plus variés:

Agriculture, Estimation des terrains, Architecture, Sculpture, Travaux publics, Construction des routes et chemins de fer, Canalisation, Distribution d'eau, Exploitation des mines et des carrières, Production de la chaleur et de la lumière, Fabrication des objets céramiques et des verreries, $\mathbf{y}$ compris les émaux et lles couvertes, Choix et préparation des substances propres à broyer, à repasser, à polir et à brunir, Fabrication des poteries et autres matériaux réfractaires, Purification des teintures et des peintures minérales ainsi que des matières propres au foulonnage et au dégraissage, Propriétés des sels natifs, des terres salines, Guément et composition des eaux thermales, Propriètés des médicaments fournis par les roches, Composition des eemmes et des pierres précieuses, Exploitation des métaux natifs et des minéraux wo talliques, etc. 


\section{J. ROTHSCHILD, Eiditeur, 13, Rue des Saints-Pères, Paris}

\section{VIENT DE PARAITRE LA $3^{\circ}$ EDITION \\ LE CHEVAL ET SON CAVALIER HIPPOLOGIE ET ÉQUITATION}

Par le Comte J. DE LAGONDIE

Ancien Colonel d'élat-najor

École pratique pour la connaissance, l'éducation, la conservation, l'amélioration du cheval de course, de chasse, de guerre; d'après les récentes publications anglaises sur le turf, arec des tables généalogiques et nombreuses additions au point de vue du cheval français. Un fort volume avec nombreuses gravures, imprimé sur papier teinté; relie avec luxe en toile, tranches en couleur.

\section{Prix : 7 Francs 50}

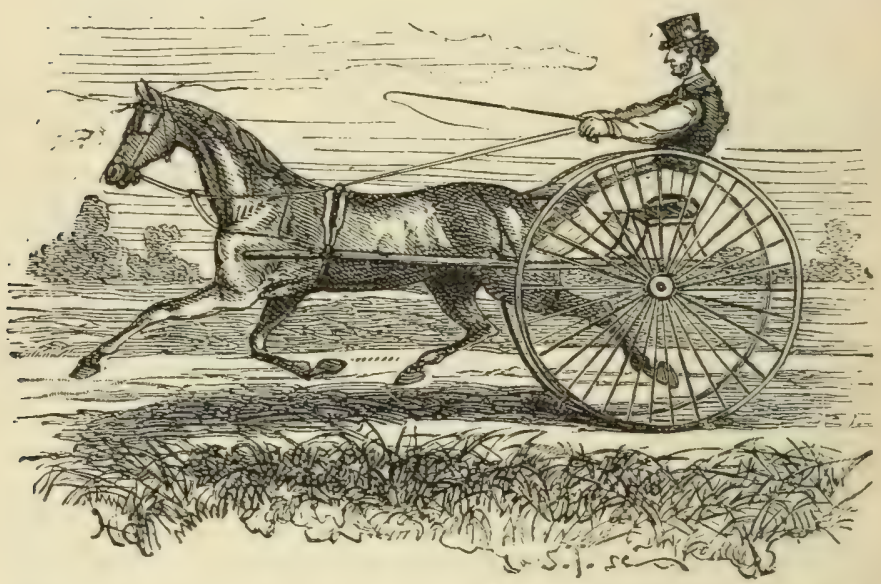

Sommaime de L'ouvrage. - Courses de Chevaux - Handicaps, Paris, Cheval de course, Origine, Vitesse, Pureté du sang, Forme extérieuro, Haras, Élevage, Écuries, Sellerie, Ferrure, Entraînement, Poulinière, Dressage, Pistes, Chef, Groom, Jockey, Frais d'élevage.

Courses de haies et Steeple-Chase. - But, Règlement, Poids, Hippodrome, Entrainements.

A la Queue des Chiens. - Hunter, Écurie et Achat, Dressage, Entrainements.

Conrses au trot. - Trotteur, Cavalier, Terrain, Entrainements.

Théorie et pratique de l'élève du Cheval de Course. - Unions, Croisement, čhoix de Poulinière et d'Étalon, Liste d'Étalons modernes.

Entrainement pour les Pédestrians ; Hippiatrique et Equitation. - Équipement, Équitation des dames, Chevaux d'attelage, Pansagc, Nourrituro: Tondre, brûler et faire les crins; Vices d'écuries, Voitures, Harnais. 
J. RoTHSCHILD, Editeur, 13, Rue des Saints-Pères, Paris

\section{LES POISSONS}

\section{D'EAU DOUCE ET DE MER}

Synonymie - Description - Mœurs - Frai - Pèche - Iconographie des especes composant plus particulièrement la Faune d'Europe, par H. Gervais et R. Boulart, aides naturaliste's au Mruseum, avec une Introduction, par Pacl iervais, Membre de 'Inslitut, pioféssun' an Mruséun. - Trois volumes grand in- $8^{\circ}$, arec 850 pages de texte, 130 vignettes et 260 splendides Chromotypographies.

CHAQUE VOLUME SE VEND SEPAREMENT :

Tome I. Les Poissons d'Eau douce. -Prix 30 Francs; relié 35 Franes. - Ir. Les Poissons de Mer (1'0 partie). - Prix $45 \mathrm{Fr}$; relie $50 \mathrm{Fr}$. - III. Les Poissons de IIer (20 partie). - Prix $45 \mathrm{Fr}$.; relié $50 \mathrm{Fr}$.

\section{LA PISCICULTURE}

Fluviale et maritime en France, par Jules Pizzetta et M. DE BoN Description des Poissons - Pêche - Lois - Repeuplement des Rivieres - Elevage des Poissons, des Ecrevisses et des Sangsues. Suivie d'un traite sur l'Ostreiculture en France. Ln volume de 500 pages, orné de 212 gravures, relié ........ Prix 4 Fr.

\section{PATHOLOGIE DES POISSONS}

Traité des Maladies, des Monstruosités et des Anomalies des CEufs et des Embryons, par Michel Girdwoyx. - Publicalion de luxe pioinée par la Sociélé d'Acclimalation die Paris. - Ourrage orne de 11 planches lithographiques dessinees par l'auteur d'apres nature.TIn vol. in-folio. Prix ........... $20 \mathrm{Fr}$

La Culture des Plages maritimes, par If. DE LA BLAnchere.Preface par Coste (Membie de l'Instiluit) A rec 70 gravures, relie, 3 Fr.

L'Aquarium d'eau douce et d'eau de mer. - Ses Formes, Préparation, P'opulation, Salubrite, Plantes, Poissons, Reptiles, Mollusques, Crustacés, Inscetes, Infusoires, par Jules PIzzetta; avec une Introduction

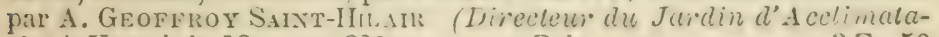
(ioii). Un vol. in-18, avec 220 gravures. Prix. . . . . $3 \mathrm{Fl} .50$

Le Monde microscopique des Eaux, par Jules Grand. - La Y e animale duns l'Eau. - Comment on ubserve le déreloppemeut des infusoire:! :- L'animalité indefinie. - I.es Tégelux mieroscoquiques. - vu cómmence la vie vègétale. - litudes au burd d'un fossé. - I'etites canses, grands effets. - La Microntologie. - I.e fond de lit mer. - Les fussiles microscopiques. - Ia vie minerale vue au microscope. - Un vol. in-18, orne de 70 gravures, relić. . $3 \mathrm{Fr} .50$ 


\section{HORTICULTURE - BOTANIQUE}

\section{Les Orchidées Botanique, classification, géographie, collections, cul- détaillée de toutes les especes cultivées en Europe, par E. de Purd Un volume avec 242 vignettes et 50 chromos, $\mathbf{3} 0 \mathrm{fr}$; relié $25 \mathrm{FR}$.

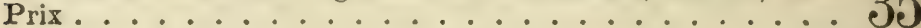
L'Olivier Histcire, botanique, physiologie, culture, produits, usage, l'Olivier, par le professeur A. Coutance. - Un volume in-8 $15 \mathrm{Fr}$ avec 120 gravures.

Les Champignons Histoire, description, emploi, culture, usage vignettes et 60 chromolithographies, Prix: $\mathbf{3 0}$ fr.; relié. . . 35

Arboretum et Fleuriste de la ville de Paris. - Description, seaux, et des plantes herbacées et frutescentes de plein air et de serre employèes dans l'ornementation des villes, pares et jardins, par 50 FR. A. Alphand, Directeur des Travaux de l'aris. Un volume in-folio.

Les Ravageurs des Vergers et des vignes. - Histoire moyens de les combattre arec une Etude sur le Phylioxéra, 3 Fr. 50

Les Maladies des Plantes cultivées des arbres fruitiers Bary, Berkeley, Hartig, Sorauer, ete, par M. A. d'Arbors DE JunAINvILLE, Sous-Inspecteur des forêts, et J. VFSQUE, Préparateur au Muséum et à l'Instiut agricole. Un volume avec 48 vignettes et 7 planches en couleur's.

Les Diseaux utiles et nuisibles aux champs, jardins, forêts, Les Oiseaux utiles et nuisibles plantations, vignes, par H. de la Blanchicre. - 3e édition revue et augmentée. Un volume avec 150 vignettes, relié toile, tranches ronges

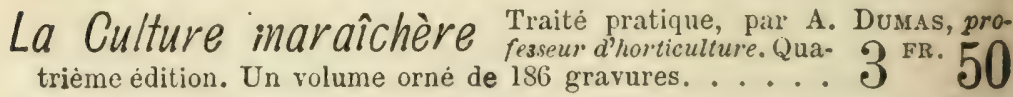
La Vigne dans le Bordelais Commerce, culture, histoire

PETrT-LAFITTE, professeur d'arboriculluture du de artement de la $12^{\text {tr. }}$ Gironde. - Un fort volume in $8^{\circ}$, illustré de 75 gravures ..

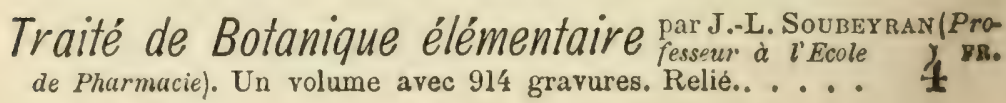




\section{J. ROTHSCHIL D, Editear, 13, Rue des Saints-Pères, Paris.}

DIENT DE PARAITRE:

\section{LES RAYAGEURS}

\section{DES}

\section{VERGERS et des VIGNES}

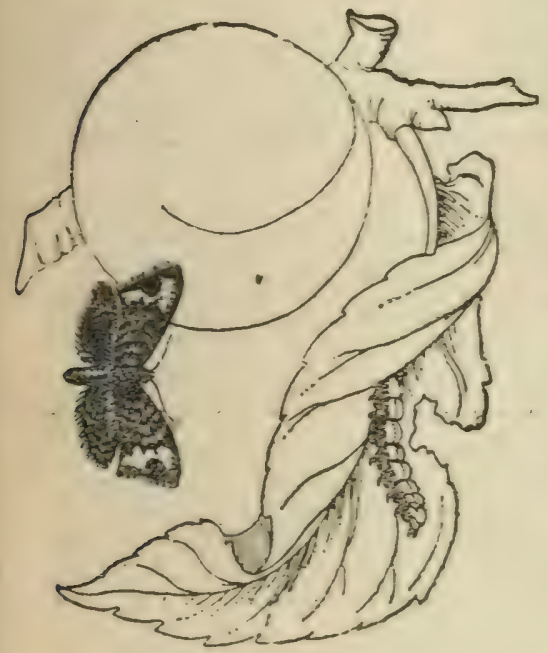

HISTOIRE NATURELLE

MOEURS - DEGATS

HOFBSS DE LES COYBITTBB

AVEC UNE ETUDE

SUE LE

PHYLLOXERA

$\operatorname{IAB}$

H. DE LA BLANCHERE

Ancien Élève de l'École fores. tuère de Viancy

On volume $2 n \cdot 18$ relie, avec 160 Gravures dessinées d'après Nalure. - Prix: 3 fr. 50.

Sans nous précecuper de classifications ni de distinctions hers nature, nous arons groupé nos Ravazei:rs des Vergers el des Vigres de la ma. nière la plus étémentaire, on partant du lieu de leurs déjüss.

Ainsi donc nous établissons dabord deux grandes divisions, nécessitées par la différence des accidents et la différence des traitemeris: Vergers el Tigres. Puis, dans chacune de ces divisions, les chapitres sont dans l'ordre suirant: Ravageurs des racines, - des tuges, - dez branches, - des bourgeons, - des feuilles, - des Reurs, - des fruits.

En somme, que reut le lecteur?... Reconnaitre le mal, dabord; aj. prendre la cause, ensuile; trouver l'indication du remède, Iorsqu'on en connait. Passé cela, tout ce que rous lui direz est inctile, auisible mêma, et, - soyons franc - ennuyeux pour lui!

En se promenant dans son Verger, il aperçoit des brindilles úl pea. ient, sèches et coupées à l'extrémité des branches de ses meilleürs r:amiers... - Qu'est-ce cela?

Il s'approche de l'arbre, atteint une des brindilles.

- 'Tiens !... C'est un bourgeon coupé et Aétril...

Il revient à notre petit livre:

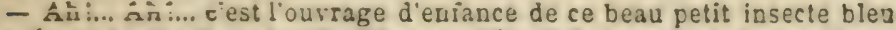
vucé... ah ! on le troure ici!.. Bien; on détruit la larre de tello msaière..

Vollà ce que reut le kecteur des Pavageurs. 


\section{J. R0THSCHILD, Ėditeur, 13, Rue des Saints-Pères, Par}

\section{HORTIGULTURE - BOTANIQUE}

L'Art des Jardins histoire, thejorie, pratique de la com baron Errouf. - 2 Émitox. (Sous presse). Publication de luxe inornée de vignettes représentant des plans de Jardins anciens et mod nes, petits Jardins, Pares modernes, Jardins de ville, Fiosques, Maiso d'habitation, Ponts, Tracés, Détails pittoresques, Accidents de terraiı Arbres, Effets d'arbres, Plantes ornementales, etc.; augmentèe des p] jolis Squares de la ville de Paris avec leur disposition des plantes, des plans des Pares et Jardins les plus réussis - de MII. Alphand, comte Choulot, Barillier-Deschamps, Najer, Kemp, Neumann, Hirschf, etc., etc., pourant tous servir d'excelleuts modeles. - Ourrage esst tiellement pratique à l'usage de tout propriétaire de Jardin (du plus pe au plus grand $\mathbf{P a r c})$, des Ingénieurs, Horticulteurs, Régisseurs, Arc' tectes, etc., etc.

\section{L'Esprit des Fleurs Symbolisme et Science. Par Mme Ensreli} Un rolume in- $4^{\circ}$ avec 60 pages de compositions en chromo, représenta environ 400 plantes imprimees en 14 couleurs. Le texte entouré de cadi de Sisron de Colixes, est tiré en 5 couleurs. Publication de grand lu formant une Flore illustree et en même temps un Langage de fleus Prix broché 15 franes; relié avec fers specciaux, tranches dorées

Les Plantes à Feuillage coloré. - Recueil des plus remarquab servant à la décoration des pares et des jardins, $c$ serres et des appartements; prècédé d'une introduction générale sur couleur du feuillage, par Charles Naudis, Membre de l'Institut. De volumes in-S, avec 120 chromotypographies et 120 vignettes.

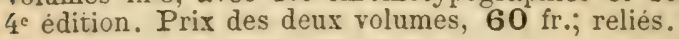

\section{Les Plantes alpines Station, culture, emploi, description $\dot{c}$ remarquables, par B. Vertor, Chef de l'ecole botanique du Iluséum. I volume avec 50 chromotypographies et 78 gravures sur bois. 35 I 2 édition, 30 fr.; relié . . . . . . . . . . . 00}

Les Fougères et les Selaginelles choix des plus rem: tion des serres, pares, jardins et salons, avec histoire hotanique, pit

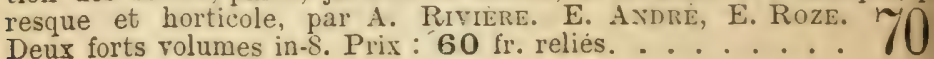

Les Roses Recueil des plus jolies espèces et rariètés cultivées d'après nature, dans les cultures de M. MIargottin fils. - Ouvrage para sant mensuellement en fascicules grand in-folio, aree 5 chromos et tex descriptif, à partir de Mars 1884.

Les Palmiers Histoire botanique, description, emploi, culture, as connues, par Oswald de Kerciore De Dexterghiex. Un volume gra

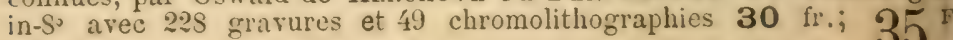
reliè . 


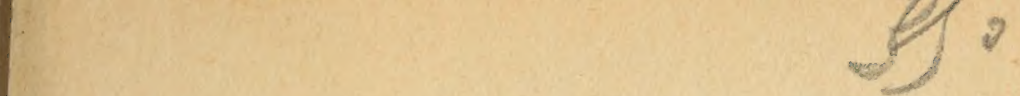



SD

Puton, Alfred

431

L'aménagement des forêts

P8

1884

PUTON, A.

SD

Forestu
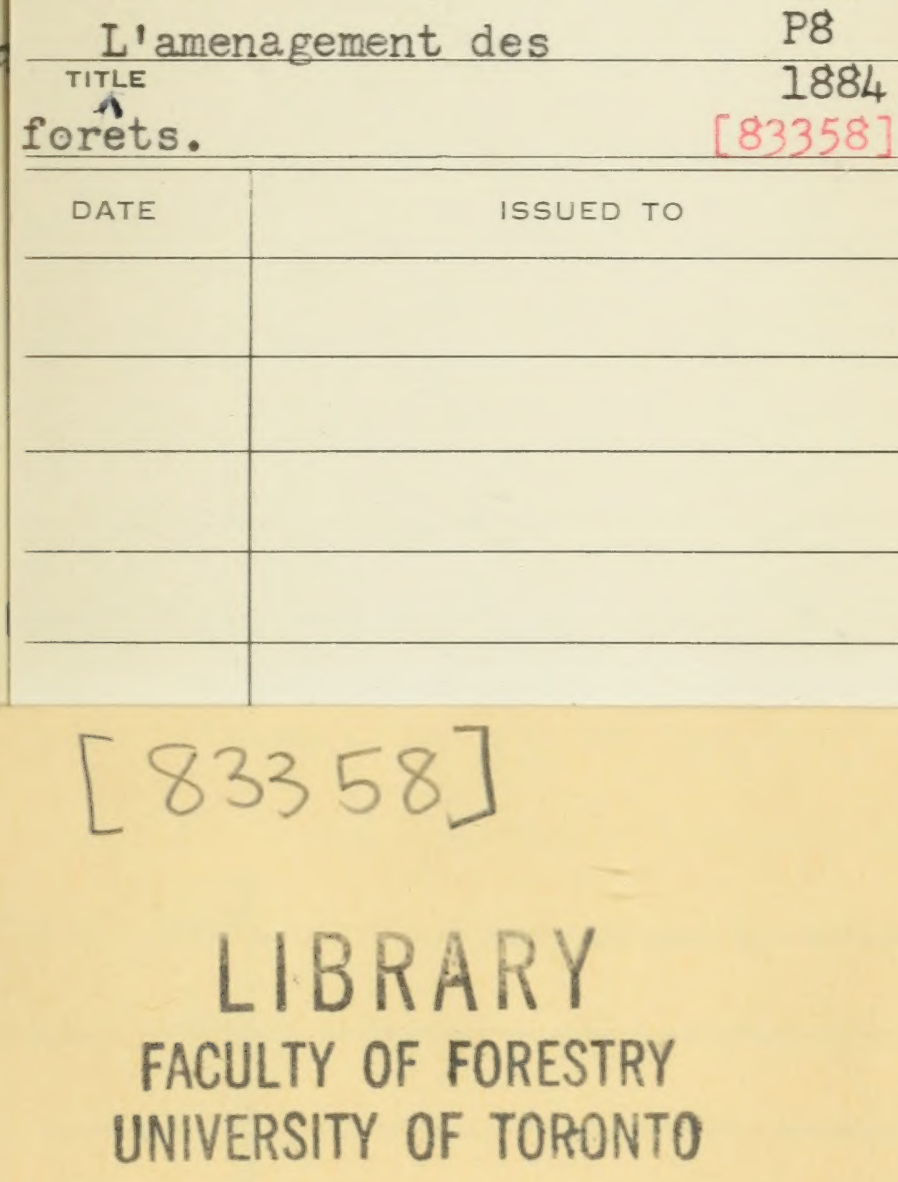


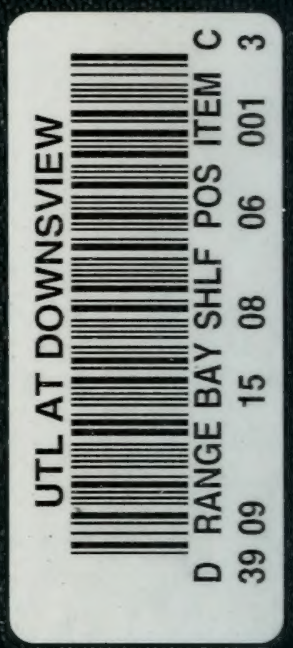

